\title{
CONSTRUCCIÓN Y EVALUACIÓN DE UN ELECTRODO POTENCIOMÉTRICO SELECTIVO A IONES Hg(II) UTILIZANDO NUEVOS IONÓFOROS
}

Tesis que para obtener el título de doctor en ciencias (Química) presenta:

\section{JORGE JUÁREZ GÓMEZ}

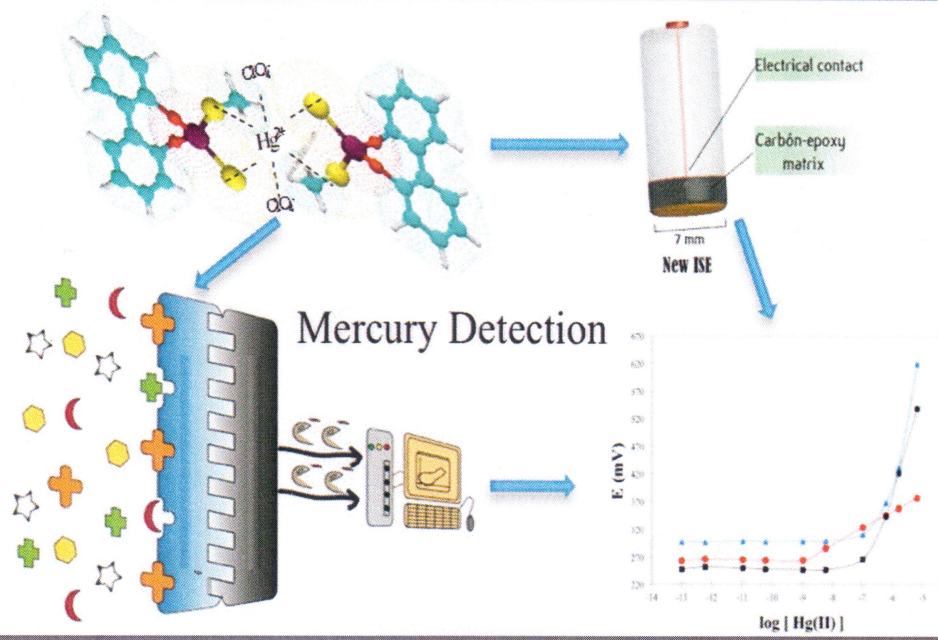

Directora: Dra. María Teresa Ramírez Silva

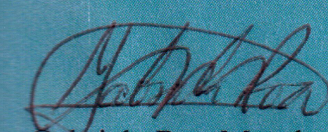
Dra. Gabriela Roa Morales Centro de Investigación en Químic Sustentable UAMeX-UNAM

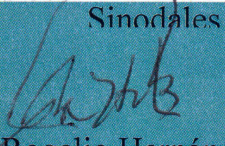

Dr. Pablo Rogelio Hernández Rodríguez Departamento de Ingeniería Bioelectrónica CINVESTAV

Dr. Miguel Ángel García Sánchez Departamento de Quimica UAM-Iztapalapa

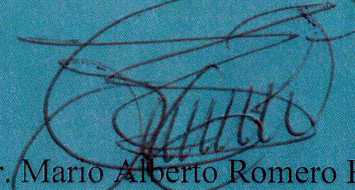

Dr. Mario Alterto Romero Romo Depdxtamento de Materiales UAM-Azcapotzaleo 


\section{Tabla de contenido}

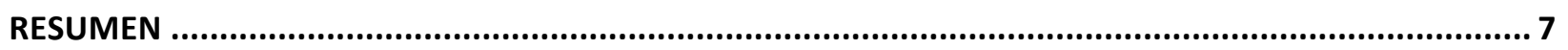

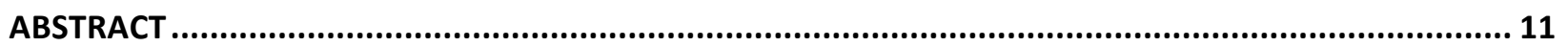

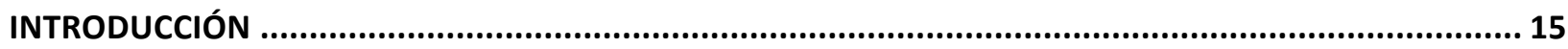



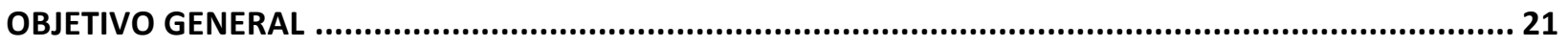

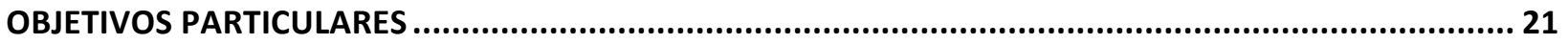

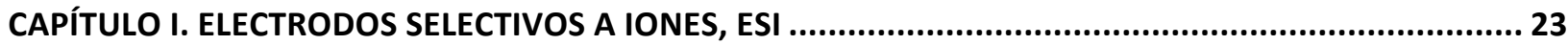

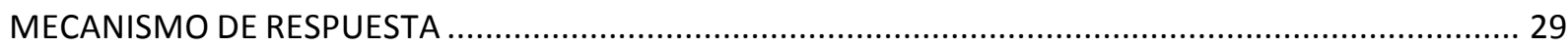

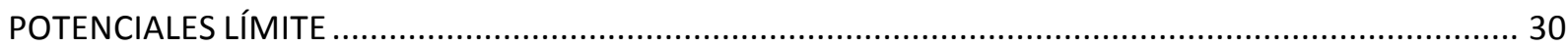

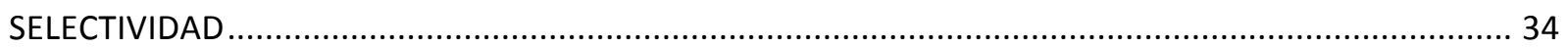



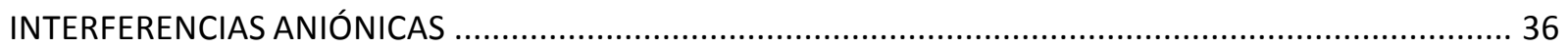

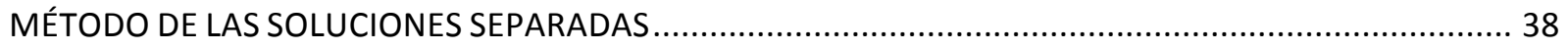

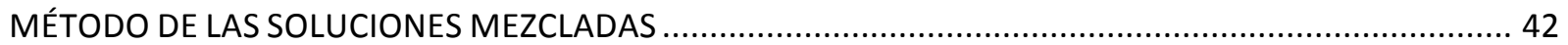

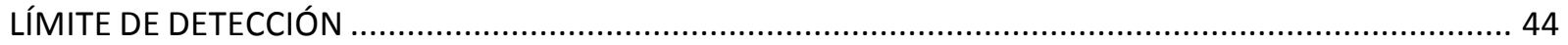

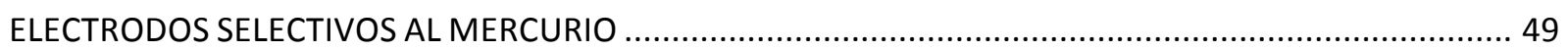

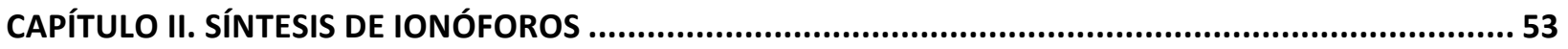

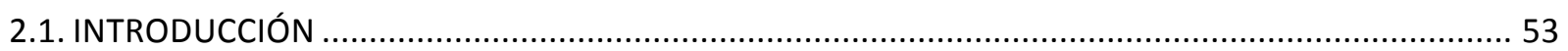

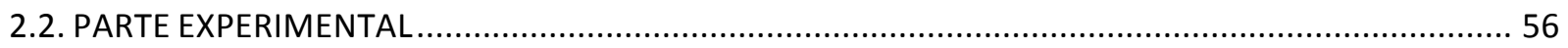



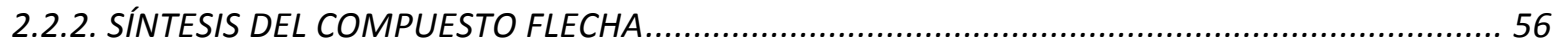

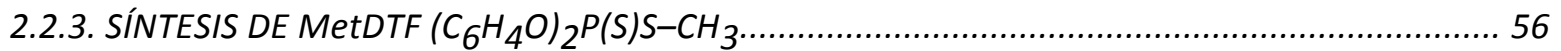

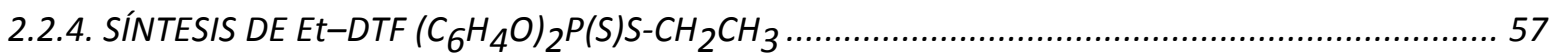

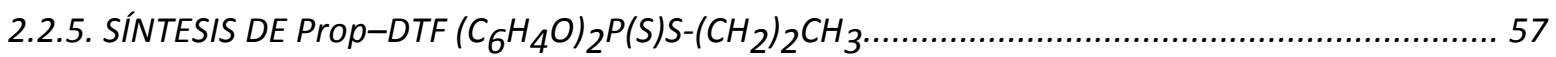

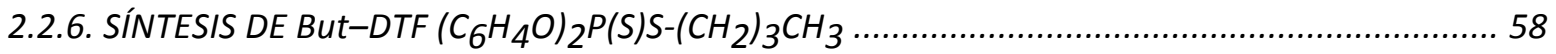

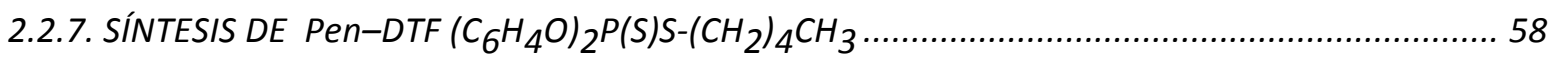

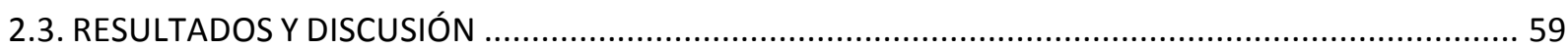

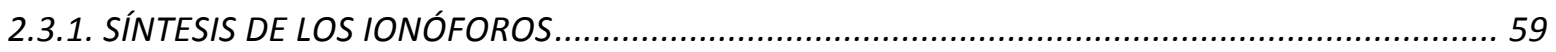

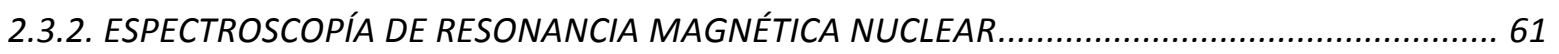

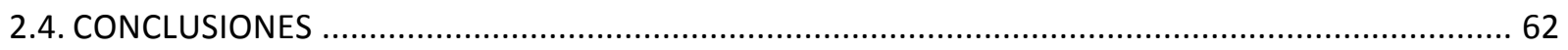

CAPÍTULO III. ELECTRODO POTENCIOMÉTRICO DE CONTACTO INTERNO SÓLIDO Y DE SUPERFICIE RENOVABLE BASADO EN UN NUEVO IONÓFORO DITIOFOSFORADO SELECTIVO A HG(II) ....................65

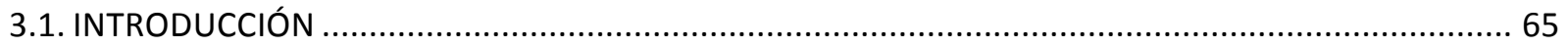

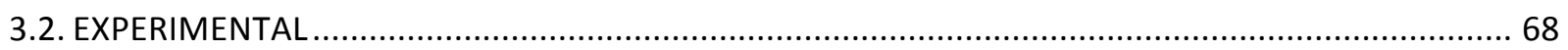

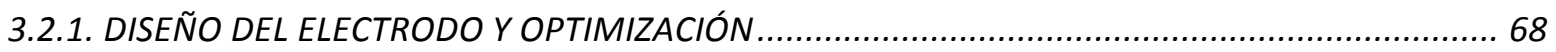

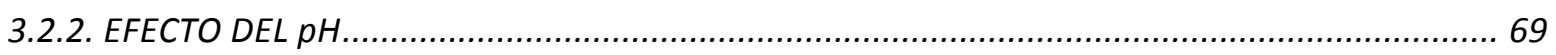

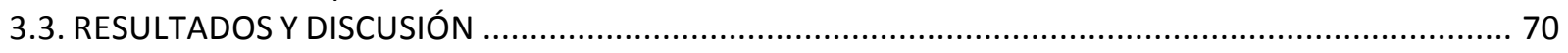

3.3.1. OPTIMIZACIÓN DE LA COMPOSICIÓN DEL ELECTRODO ….............................................. 70

3.3.2. REPRODUCIBILIDAD Y EFECTO DE LIJAR LA SUPERFICIE DEL ELECTRODO.......................... 72

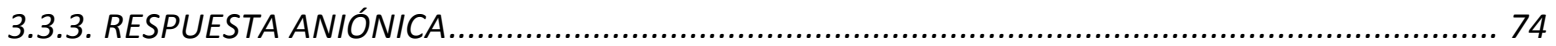






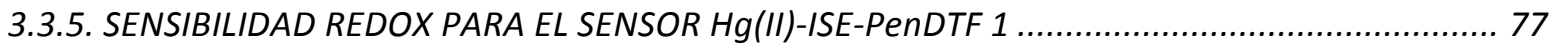

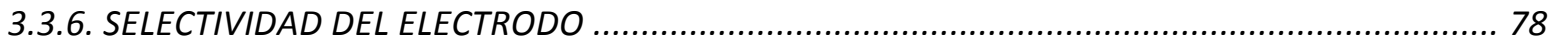





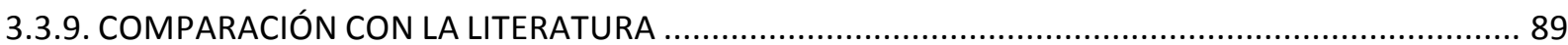

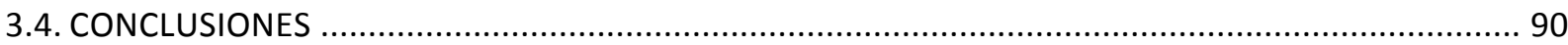

CAPÍTULO IV. NUEVO ELECTRODO POTENCIOMÉTRICO DE CONTACTO INTERNO SÓLIDO PARA LA DETECCIÓN SELECTIVA DE MERCURIO A NIVELES NANOMOLARES ............................................93

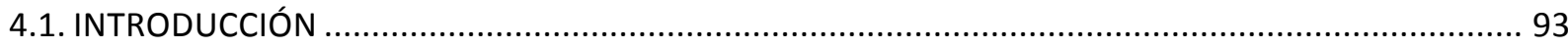

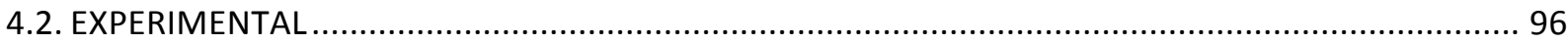



4.2.2. CONSTRUCCIÓN DEL ELECTRODO "COMPOSITE" DE CONTACTO INTERNO SÓLIDO ............... 97

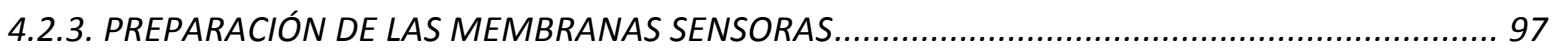

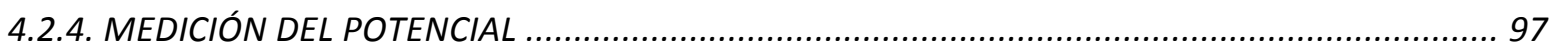

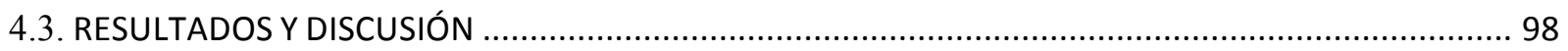

4.3.1. EFECTO DE LA CADENA ALQUÍLICA Y DEL ACONDICIONAMIENTO DE LA MEMBRANA.......... 98

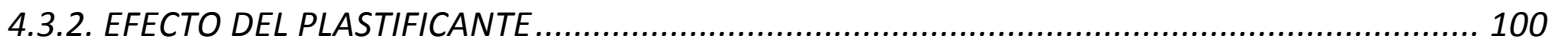

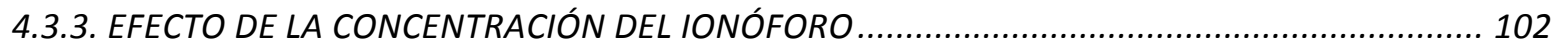

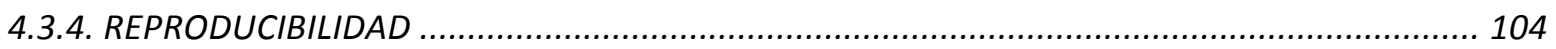

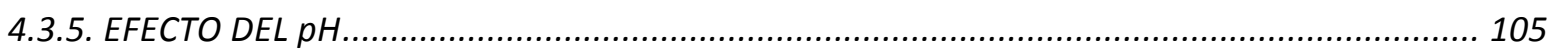

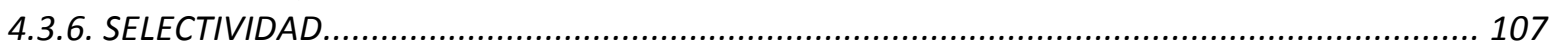

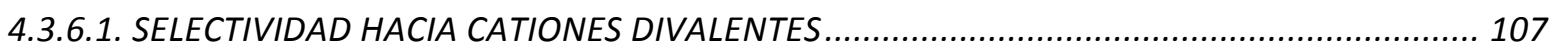

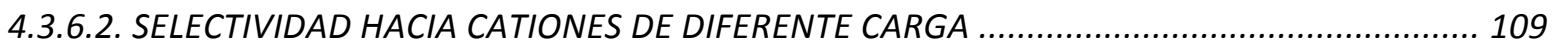

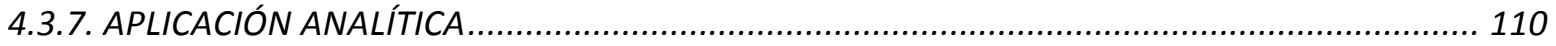

4.3.8. COMPARACIÓN CON LA LITERATURA

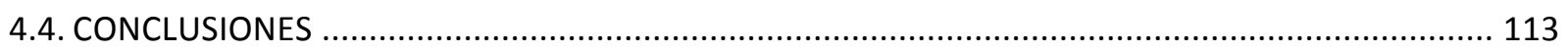

CAPÍTULO V. DISEÑO DE CONTACTO INTERNO SÓLIDO BASADO EN UNA MATRIZ CARBÓN-EPOXY

PARA LA CONSTRUCCIÓN DE ESI QUE USA UN IONÓFORO COMERCIAL........................................ 116

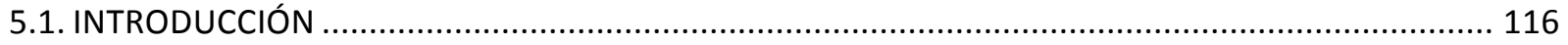

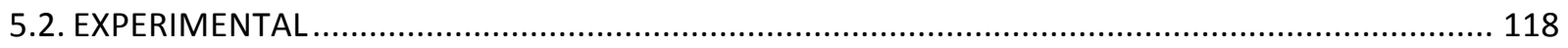

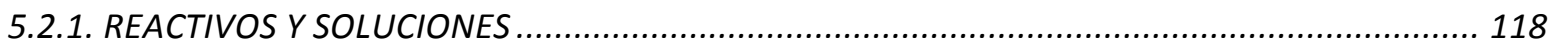

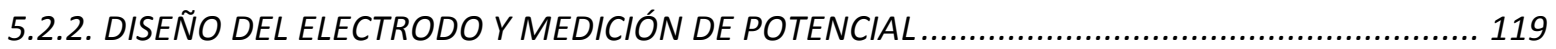

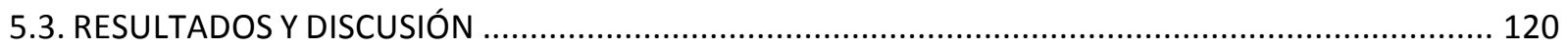

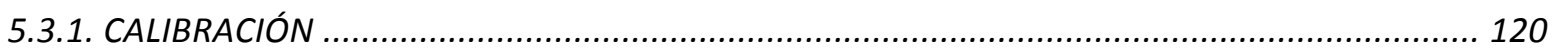

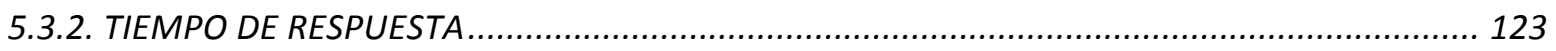

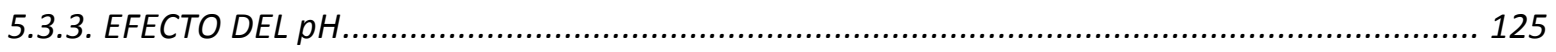

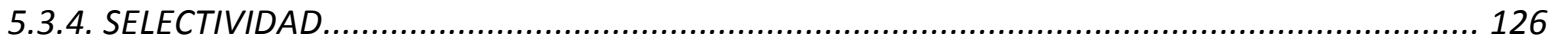

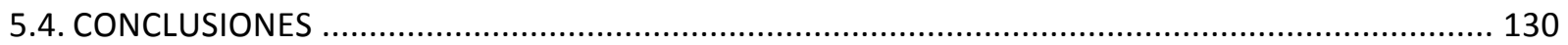

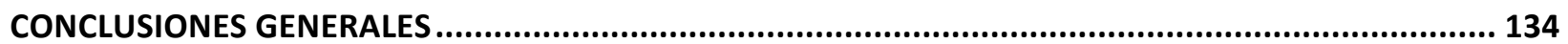

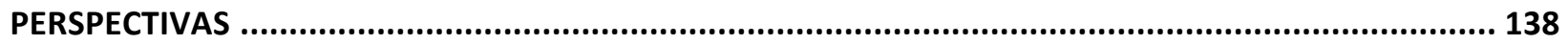

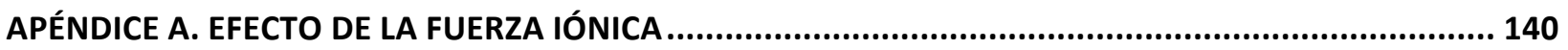

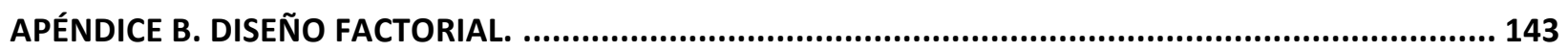



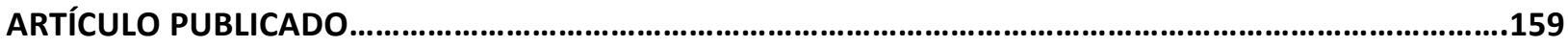


$\mathcal{A}$ mis padres, y mis hermanos

Al término de éste proyecto tan importante en mi vida profesional, quiero agradecer a todas las personas que contribuyeron a su realización. En primer lugar a mi familia, a mis padres y mis hermanos por su constante apoyo; a mi asesora Tere Ramírez por sus sabios consejos y vasta experiencia; a todos mis amigos y compañeros de la UAM-I por su grata compañía a lo largo de este tiempo; a todos mis amigos fuera de la universidad por todos los momentos de desestrés los fines de semana; a mis sinodales por aceptar evaluar esta tesis; a CONACYT por la beca para estudios de posgrado 240838 y a la vida por saber ponerme en los lugares correctos, por que cuento con la major familia que se puede tener, con los mejores padres que pueden existir, con los mejores amigos que se puedan encontrar y con los mejores maestros que se pueden pedir. 


\section{RESUMEN}




\section{RESUMEN}

En esta investigación se describe la síntesis de compuestos químicos con características apropiadas para ser utilizados como ionóforos en membranas poliméricas para electrodos selectivos a iones mercurio(II). La síntesis se basa en la introducción de un grupo alquilo lineal al compuesto O,O'-(2,2'-bifenilen)ditiofosfato de potasio (compuesto flecha). De esta manera se obtuvo la serie de cinco ionóforos $\mathrm{CH}_{3}\left(\mathrm{CH}_{2}\right)_{n} \mathrm{~S}_{2} \mathrm{P}\left(\mathrm{OC}_{6} \mathrm{H}_{4}\right)_{2}(\mathrm{n}=0-4)$, en donde va en aumento el tamaño de la cadena, desde metilo hasta $n$-pentilo. También, se presentan estudios potenciométricos de un novedoso electrodo selectivo de iones (ESI) de contacto interno sólido basado en un nuevo ligante ditiofosforado O,O'-(2,2'-bifenilen)ditiofosfato de pentilo (PenDTF) que presenta una alta selectividad hacia $\mathrm{Hg}$ (II), el que en presencia de iones divalentes, mostró una buena respuesta Nernstiana hacia el $\mathrm{Hg}(\mathrm{II})(33.81 \pm 1.24) \mathrm{mV}_{\text {década }}{ }^{-1}$ sobre un amplio intervalo de concentraciones $\left(5.35 \times 10^{-7}-1.00 \times 10^{-2}\right) \mathrm{mol} \mathrm{L}^{-1}$, el límite de detección fue de $(4.24 \pm 2.48) \times 10^{-7} \mathrm{~mol} \mathrm{~L}^{-1}$. Debido a su diseño, el electrodo puede ser utilizado para varios experimentos renovando la superficie de contacto con papel abrasivo sin observar desviación significativa en su respuesta con un coeficiente de variación menor al 3\%, pudiendo ser probado en el intervalo de $\mathrm{pH}$ de $0-5$; el electrodo fue utilizado satisfactoriamente como electrodo indicador en la valoración potenciométrica de iones $\mathrm{Hg}(\mathrm{II})$ con EDTA. Con el resto de los ionóforos se construyeron ESI con una matriz polimérica compuesta de ionóforo/plastificante/PVC/NaTPB. Al final se obtiene un sensor que permite la medición continua de mercurio a niveles nanomolares en un amplio intervalo de concentraciones $\left(1.0 \times 10^{-}\right.$

${ }^{9}$ a $\left.1.0 \times 10^{-1}\right) \mathrm{mol} \mathrm{L}^{-1}$, con un límite de detección de $(9.1 \pm 0.8) \times 10^{-10} \mathrm{~mol} \mathrm{~L}^{-1}$ y con una 
respuesta Nernstiana de $(29.8 \pm 0.4) \mathrm{mV} \mathrm{década}^{-1}$. El ESI puede ser utilizado en un intervalo de $\mathrm{pH}$ de $0-6$, además presenta una alta selectividad hacia iones mercurio aun en la presencia de iones interferentes y puede ser utilizado como electrodo indicador en la valoración potenciométrica de mercurio con EDTA ya que es comparable con electrodos comerciales de su misma clase. Finalmente, se comparan los sensores con uno construido con el ionóforo comercial 1, 10-Dibenzyl-1, 10-diaza-18-crown-6 selectivo a $\mathrm{Hg}(\mathrm{II})$, dando como resultado que los propuestos aquí presentan muchas ventajas, por ejemplo: un mejor límite de detección, un mayor intervalo de linealidad, un intervalo de $\mathrm{pH}$ más amplio, además de ser más robustos y menos costosos. 
ABSTRACT 


\section{ABSTRACT}

This thesis describes the synthesis of chemicals suitable for using as ionophores in polymeric membranes for ion-selective electrodes for mercury (II). The synthesis is based on the introduction of an alkyl linear group into the compound O, O'- (2,2'-biphenylene) dithiophosphate potassium (arrow compound). Thus the synthesis the series of five ionophores $\mathrm{CH}_{3}\left(\mathrm{CH}_{2}\right)_{n} \mathrm{~S}_{2} \mathrm{P}\left(\mathrm{OC}_{6} \mathrm{H}_{4}\right)_{2}(\mathrm{n}=0-4)$ was achieved, through increasing the chain from methyl to n-pentyl. Also, the potentiometric studies of a novel solid contact ion-selective electrode based on a new dithiophosphored ionophore O,O'-(2.2'-biphenylene) pentyl dithiophosphate (PenDTF) are presented. The ISE has a high selectivity to $\mathrm{Hg}(\mathrm{II})$ in the presence of divalent ions, the electrode showed a good Nernstian response to $\mathrm{Hg}$ (II) $(33.81 \pm 1.24) \mathrm{mV}_{\text {década }}{ }^{-1}$ over a wide range of concentrations $\left(5.35 \times 10^{-7}-1.00 \times 10^{-2}\right) \mathrm{mol} \mathrm{L}^{-1}$, the detection limit was $\left(4.24 \times 10^{-7} \pm\right.$ $\left.2.48 \times 10^{-7}\right) \mathrm{mol} \mathrm{L}^{-1}$. Due to its design the electrode can be used for several experiments renewing the contact surface with sandpaper without observing significant deviation in its response with a coefficient of variation less than $3 \%$ and could be tested in the $0-5 \mathrm{pH}$ range, the electrode was successfully used as an indicator electrode for the potentiometric titration of $\mathrm{Hg}(\mathrm{II})$ ions with EDTA. The construction of a new ion selective electrode (ISE) for the potentiometric determination of mercury ions is presented, using a support matrix and carbon-epoxy composite with a polymer matrix of ionophore / plasticizer / PVC / NaTPB. This sensor allows continuous measurement of mercury at nanomolar levels in a wide concentration range $\left(1.0 \times 10^{-9}\right.$ to $1.0 \mathrm{x}$ $\left.10^{-1}\right) \mathrm{mol} \mathrm{L}^{-1}$, with a limit of detection $(9.1 \pm 0.8) \times 10^{-10} \mathrm{~mol} \mathrm{~L}^{-1}$ and a Nernstian response $(29.8 \pm$

0.4) $\mathrm{mV}$ decade $^{-1}$. The ISE trus characterized can be used in the 0-6 pH range, also has a high 
selectivity for mercury ions even in the presence of interfering ions and can be used as an indicator electrode for potentiometric titration of mercury with EDTA since it is comparable to commercial electrodes of the same class. 


\section{INTRODUCCIÓN}




\section{INTRODUCCIÓN}

En la última década, los problemas de contaminación han adquirido tal magnitud que la sociedad ha tomado cada vez más conciencia de los riesgos actuales, y más aún, de los potenciales. Los que toman las decisiones han mostrado una creciente voluntad política para resolver estos problemas, todo esto en resultado a la presión social generada. Ahora, es necesario que especialistas altamente calificados ofrezcan soluciones realistas.

Todos hemos escuchado alguna vez en la radio, visto en la televisión o leído en prensa e internet sobre el deterioro ambiental en México y el mundo, y esto ha contribuido a formar opiniones sobre el problema. Sin embargo la mayoría de las veces, estos medios no proporcionan una cultura formal sobre el tema e inducen a la adopción de soluciones que si bien son populares no siempre son adecuadamente técnicas, económicamente viables y socialmente factibles. Para poder plantear soluciones que no solamente "suenen bien" sino que además puedan ponerse en práctica y que sobre todo mejoren y preserven el medio ambiente, se debe contar, entre otras cosas, del conocimiento básico, técnico y global del problema y de las posibles opciones de solución.

A lo largo del tiempo, la calidad del agua potable ha sido determinante para garantizar el bienestar humano. Actualmente las aguas insalubres contaminadas por acciones naturales o humanas siguen siendo un problema para las personas que se ven obligadas a usarlas, tanto en la industria agropecuaria y alimenticia e incluso para beberla. 
Generalmente, la mayor preocupación sobre la seguridad del agua es la presencia potencial de compuestos químicos, éstos se pueden presentar tanto en forma orgánica como inorgánica. Todos estos compuestos químicos son provenientes de la industria ya que se utilizan como precursores en diversos procesos.

Los metales están considerados dentro de los más dañinos y son de mucho interés dada su toxicidad. Estos elementos son, en general, los metales de transición, así como algunos de los elementos representativos como el plomo y el estaño. En los metales se incluyen a elementos esenciales para el ser humano, por ejemplo, el hierro, pero también tóxicos como el cadmio y el mercurio. La mayoría de ellos, y aún más el mercurio, presentan una gran afinidad por moléculas orgánicas que continen grupos donadores de azufre, por ejemplo proteínas, lo que favorece su bioacumulación en tejidos vivos y además de abatir la función catalítica de las enzimas que contengan grupos donadores de azufre ${ }^{1,2}$.

El mercurio, por su parte, entra al medio ambiente desde un gran número de fuentes relacionadas con el uso de este elemento por el hombre. En éstos se incluyen, los productos químicos de laboratorio desechados, baterías, termómetros, amalgamas dentales, fungicidas y productos farmacéuticos.

La toxicidad del mercurio fue demostrada en 1953 en Minamata, Japón. Se reportó un total de 111 casos de envejecimiento por mercurio y 43 muertes entre las personas que habían consumido mariscos de la bahía, que había sido contaminada con desechos de mercurio de un planta química vertidos a la bahía de Minamata. Fueron observados defectos genéticos en 19 bebés cuyas 
madres habían consumido mariscos contaminados con mercurio. El nivel del metal en el marisco contaminado fue de 5-20 partes por millón.

Entre los efectos toxicológicos del mercurio están el daño neurológico, incluyendo las siguientes afectaciones graves a la salud: irritabilidad, parálisis, ceguera o locura; ruptura de cromosomas y defectos de nacimiento. Algunas de las formas de mercurio son relativamente no tóxicas y se han usado como medicinas, por ejemplo, para el tratamiento de la sífilis. Otras formas del mercurio, particularmente los compuestos organomercuriales, son altamente tóxicas. Los compuestos organomercuriales, como el metilmercurio, etilmercurio o fenilmercurio; son sintetizados por bacterias anaerobias presentes en los sedimentos con ayuda de la metilcobalamina, un análogo de la vitamina B12, sin embargo la materia prima siempre es el mercurio inorgánico en sus diferentes formas, por ejemplo, el ión $\mathrm{Hg}(\mathrm{II})^{1-3}$.

Debido al gran impacto que el ión $\mathrm{Hg}(\mathrm{II})$ tiene en el medio ambiente y en la salud humana, existe una gran necesidad para desarrollar nuevos métodos químicos para su determinación analítica a bajas concentraciones ${ }^{4,5}$.

Entre los método clásicos que se han utilizado en la determinación de mercurio y que se caracterizan por su bajo límite de detección, se encuentran técnicas como la espectroscopía de absorción atómica (AAS), la espectrometría de masas con plasma de acoplamiento inductivo (ICP-MS) y la electroforesis capilar, entre otras, pero estas son técnicas de alto costo y no pueden ser utilizadas en análisis de campo ${ }^{6,7}$. 
Por esto ha sido necesario el desarrollo de nuevos y novedosos dispositivos analíticos que sean competitivos con los poderosos métodos de análisis en cuanto a sus bajos límites de detección, pero además sean capaces de brindar información analítica confiable, idealmente en campo y tiempo real.

Dentro de esta nueva instrumentación, se encuentran los denominados sensores químicos, que son dispositivos que se caracterizan, por su tamaño reducido, su fácil fabricación y su bajo costo 8, 9 . Las propiedades deseadas de un sensor químico, incluyen alta sensibilidad, un intervalo dinámico grande, alta selectividad a un analito, baja sensibilidad a posibles interferentes, reversibilidad de las propiedades fisicoquímicas del proceso de detección (recuperación a cortos tiempos de respuesta), y estabilidad a largo plazo. Por desgracia, un sensor, de exhibir todas estas propiedades es un ideal irrealizable en gran medida. La sensibilidad, selectividad, rapidez de respuesta, y la reversibilidad están determinadas por la termodinámica y la cinética del sensor. En especial la alta selectividad y la reversibilidad, están sujetas a limitaciones contradictorias en el diseño del sensor: la alta sensibilidad y selectividad son típicamente asociadas con interacciones fuertes, mientras que la reversibilidad requiere de interacciones débiles. En consecuencia, los sensores que muestran selectividad a solo algunas de las especies detectadas se utilizan para garantizar la reversibilidad.

Los sensores potenciométricos de iones o electrodos selectivos de iones (ESI) son un subgrupo muy importante dentro de los sensores químicos. Los ESI se caracterizan por su tamaño pequeño, portabilidad, bajo consumo de energía y por su bajo costo, que son características atractivas para aplicaciones prácticas ${ }^{8,9}$. Los ESI basados en membranas poliméricas que contienen ionóforos neutros o cargados están disponibles para la determinación de un gran 
número de iones inorgánicos y orgánicos. Sin embargo en la última década, los ESI han sido mejorados a tal punto que han dado lugar a una "nueva ola" de electrodos selectivos de iones ${ }^{9,10}$. Esto se puede atribuir a varias conclusiones importantes, como la considerable mejora en el límite de detección, nuevos materiales en las membranas, nuevos conceptos de detección, y a las mejores respuestas de los ESI.

Hacia este punto está dirigido esta tesis, ya que se propone una metodología para la construcción de electrodos selectivos de iones, en particular para la determinación potenciométrica de iones $\mathrm{Hg}(\mathrm{II})$.

A lo largo del escrito se tratan los electrodos selectivos de iones y su funcionamiento: cómo es que funciona una membrana selectiva y los procesos que ocurren en ella. Se introduce al lector en términos como selectividad y límite de detección que son términos que se utilizarán a lo largo de toda la tesis. Después se describe la ruta y métodos de síntesis de los compuestos utilizados a lo largo de la investigación, así como su caracterización por resonancia magnética nuclear (RMN) y se dan los desplazamientos químicos característicos de cada una de las moléculas. Por otra parte en el capítulo tres se propone un nuevo electrodo selectivo a iones $\mathrm{Hg}$ (II) basado en uno de los ionóforos sintetizados (capítulo 2), aquí también se exponen los resultados potenciométricos para el nuevo electrodo, además de todos los parámetros analíticos calculables. En el cuarto capítulo se exponen los resultados potenciométricos obtenidos para ESI construidos con los ionóforos restantes utilizando membranas poliméricas de distintas composiciones, en este capítulo se varían diversos parámetros como el plastificante y el ionóforos, también se incluye en la membrana un acarreador iónico para mejorar la respuesta y así obtener la composición óptima de la membrana. Y por último se comparan los resultados con un ionóforo comercial. 


\section{HIPÓTESIS}

Al mezclar las propiedades que le confire un contacto sólido a un ESI con ionóforos altamente afines al ion $\mathrm{Hg}(\mathrm{II})$ se obtendrá un electrodo potenciométrico selectivo robusto, es decir el electrodo tendrá un amplio intervalo dinámico, un tiempo de respuesta bajo, tendrá una alta selectividad, un tiempo de vida largo, un bajo límite de detección, será fácil de construir, será manejable, será miniaturizable y barato. 


\section{OBJETIVO GENERAL}

Construcción y evaluación de un electrodo potenciométrico robusto para la determinación de iones mercurio en solución acuosa, utilizando nuevos ionóforos selectivos.

\section{OBJETIVOS PARTICULARES}

- Sintetizar compuestos ditiofosforados selectivos a $\mathrm{Hg}(\mathrm{II})$ para la utilización en la construcción de ESI.

- Construir y evaluar electrodos potenciométricos selectivos a $\mathrm{Hg}(\mathrm{II})$ utilizando $\operatorname{los}$ ionóforos sintetizados.

- Estudiar el efecto que la cadena alquílica sustituyente tiene en la respuesta de los ESI.

- Seleccionar el mejor ESI con base en parámetros analíticos tales como: respuesta Nernstiana, intervalo de linealidad, tiempo de respuesta, $\mathrm{pH}$ aplicable y selectividad.

- Comparar los resultados con un ionóforo comercial. 




Lo poco que he aprendido carece de valor, comparado con lo que ignoro y no desespero en aprender. René Descartes 


\section{Capítulo I}

\section{CAPÍTULO I. ELECTRODOS SELECTIVOS A IONES, ESI}

Los sensores potenciométricos o electrodos selectivos de iones (ESI) son un importante subgrupo dentro de los sensores químicos y se caracterizan por su tamaño, portabilidad, bajo consumo de energía y bajo costo, que son características bastante atractivas cuando se habla de aplicaciones prácticas. Los ESI basados en membranas poliméricas que contienen compuestos portadores de iones, neutros o cargados (comúnmente llamados ionóforos, aunque el primer nombre refleja el hecho de que estos compuestos catalizan el transporte de iones a través de membranas hidrofóbicas) están disponibles para la determinación de un gran número de iones orgánicos e inorgánicos. Sin embargo en las últimas dos décadas, la capacidad de detección de los ESI se ha mejorado considerablemente hasta dar lugar a una "nueva ola de electrodos selectivos de iones". Esto se debe a varios avances importantes, como la mejora en los límites de detección, la ingeniería de nuevos materiales para la membrana y a una mejor comprensión teórica. 
La parte esencial de un ESI basado en un compuesto portador de iones es la membrana polimérica, que físicamente es un líquido de alta viscosidad e inmiscible en agua que comúnmente se coloca entre dos fases acuosas, es decir, entre la muestra y la solución interna (ver figura 1.1). La membrana sensora consta de varios componentes, comúnmente un ionóforo y una sal lipofílica que actúa como intercambiador iónico.

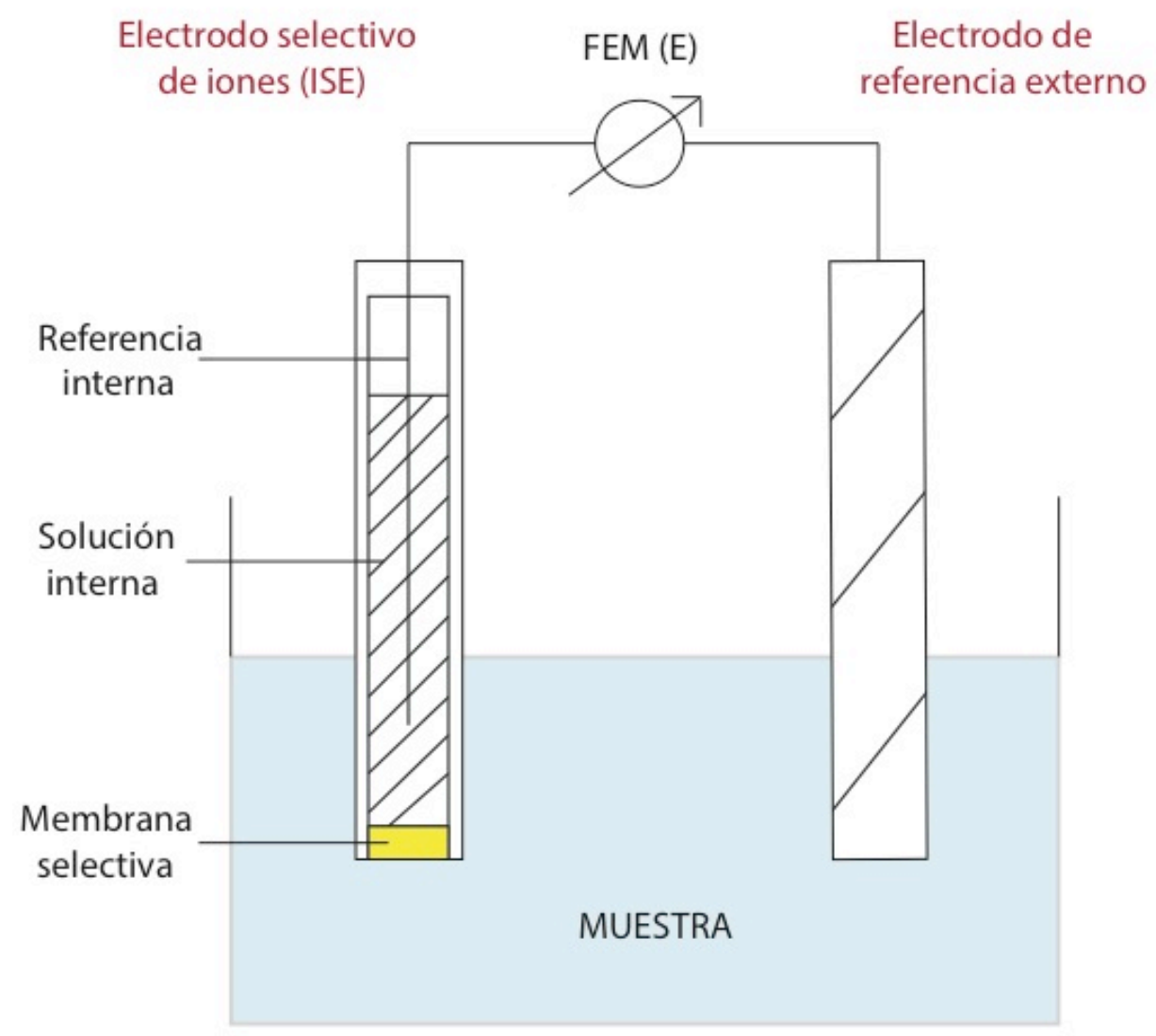

Figura 1.1. Diagrama esquemático de un circuito con un electrodo selectivo de iones.

El sensor responde a la actividad de un ion primario y generalmente cubre un intervalo de sensibilidad bastante grande, de aproximadamente 1 a $10^{-6} \mathrm{~mol} \mathrm{~L}^{-1}$, aunque con la llamada "nueva ola" el intervalo ha sido ampliado notablemente. Su selectividad está relacionada con la 
constante de equilibrio de la reacción de intercambio iónico que tiene lugar entre la fase orgánica y la fase acuosa. Y depende en gran medida de las constantes de formación de los complejos formados por el ionóforo y los iones en la fase de la membrana ${ }^{11}$ (ver figura 1.2).

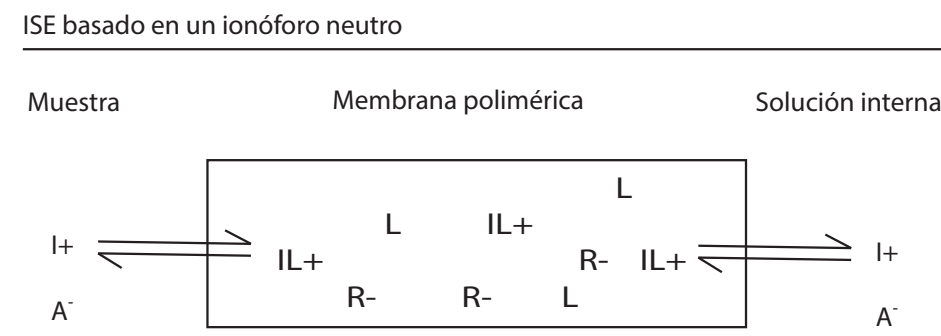

ISE basado en un ionóforo cargado

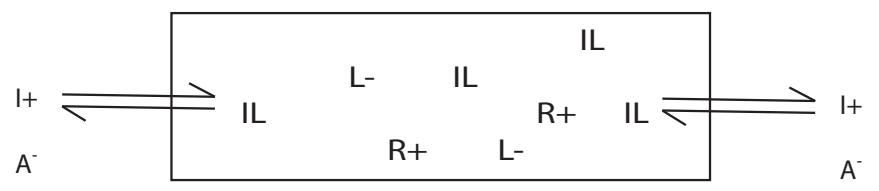

ISE basado en un intercambiador iónico

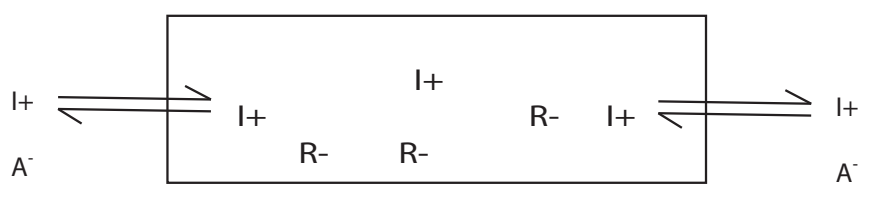

Figura 1.2. Vista esquemática de los equilibrios entre la muestra, la membrana selectiva de iones y la solución interna, para el caso especial, cuando los electrolitos de la muestra son iguales a los de la solución interna: donde $\mathrm{L}$, se refiere al ionóforo neutro, $\mathrm{R}^{-}$son los sitios aniónicos, $\mathrm{L}^{-}$es el ionóforo cargado, $\mathrm{R}^{+}$los sitios catiónicos y $\mathrm{R}^{-}$el intercambiador iónico.

Después del descubrimiento en 1964, por Moore y Pressman ${ }^{12}$ de que algunos antibióticos (ver figura 1.3) inducen el transporte de iones en las mitocondrias, Simon y Stefanac ${ }^{13}$ mostraron en 1966 que el fenómeno se debe principalmente a la formación selectiva de complejos entre 
estos compuestos y ciertos cationes. Estos autores introdujeron el primer ESI basado en un ionóforo neutro y demostraron que estos antibióticos producen selectividades in vitro similares a las observadas in vivo.

Casi al mismo tiempo, Pedersen ${ }^{14}$ en 1967 y Lehn ${ }^{15}$ en 1969 sintetizaron poliésteres macrocíclicos y compuestos macroeterobicíclicos (ver figura 1.3) y mostraron que actúan como agentes complejantes para iones de metales alcalinos y alcalino térreos. En los años siguientes se propusieron un gran número de ionóforos sintéticos y naturales y sus complejos. La tercera contribución importante al desarrollo de los ISE modernos es la proveniente de Shatkay ${ }^{16,17}$ y Ross ${ }^{18}$, que introdujeron las membranas poliméricas. Ellos propusieron un electrodo selectivo a $\mathrm{Ca}^{2+}$ basado en un ácido organofosfórico lipofílico (ver figura 1.3) y como la parte más importante de esta investigación se encuentra el uso de cloruro de polivinilo (PVC) que fue rápida y ampliamente aceptado $\mathrm{y}$, a pesar de que se han propuesto muchas más matrices poliméricas, la de PVC sigue siendo la matriz estándar para la construcción de ESI.

Con base a los resultados anteriores y en busca de más aplicaciones para los ESI, se comenzó una búsqueda intensiva de ionóforos selectivos para cationes, mientras que el desarrollo de portadores de aniones fue mucho más lento. Pocos años después de que se publicara el primer ionóforo selectivo a $\mathrm{Ca}^{2+}$, se desarrollaron bastantes ESI que contenían ionóforos neutros para la determinación selectiva de una serie de metales alcalinos y alcalino térreos y algunos otros cationes (ver tabla 1.1). Todos los ionóforos eran compuestos no macrocíclicos, y así se desmintió la idea inicial de que los agentes complejantes deberían ser macrocíclicos. Los éteres corona conocidos hasta ese momento no eran adecuados para su uso en membranas ESI, debido a que no eran lo bastante lipofílicos y además tenían una selectividad limitada. Los criptandos, aunque altamente selectivos, también carecían de lipofilicidad y además su complejación era 
lenta y esto era un obstáculo para su aplicación en membranas sensoras ${ }^{19}, 20$. Después se realizó un gran número de estudios en la química de compuestos macrocíclicos y la química supramolecular evolucionó, pero por desgracia no se aplicó lo suficiente en sensores químicos ${ }^{21}$.
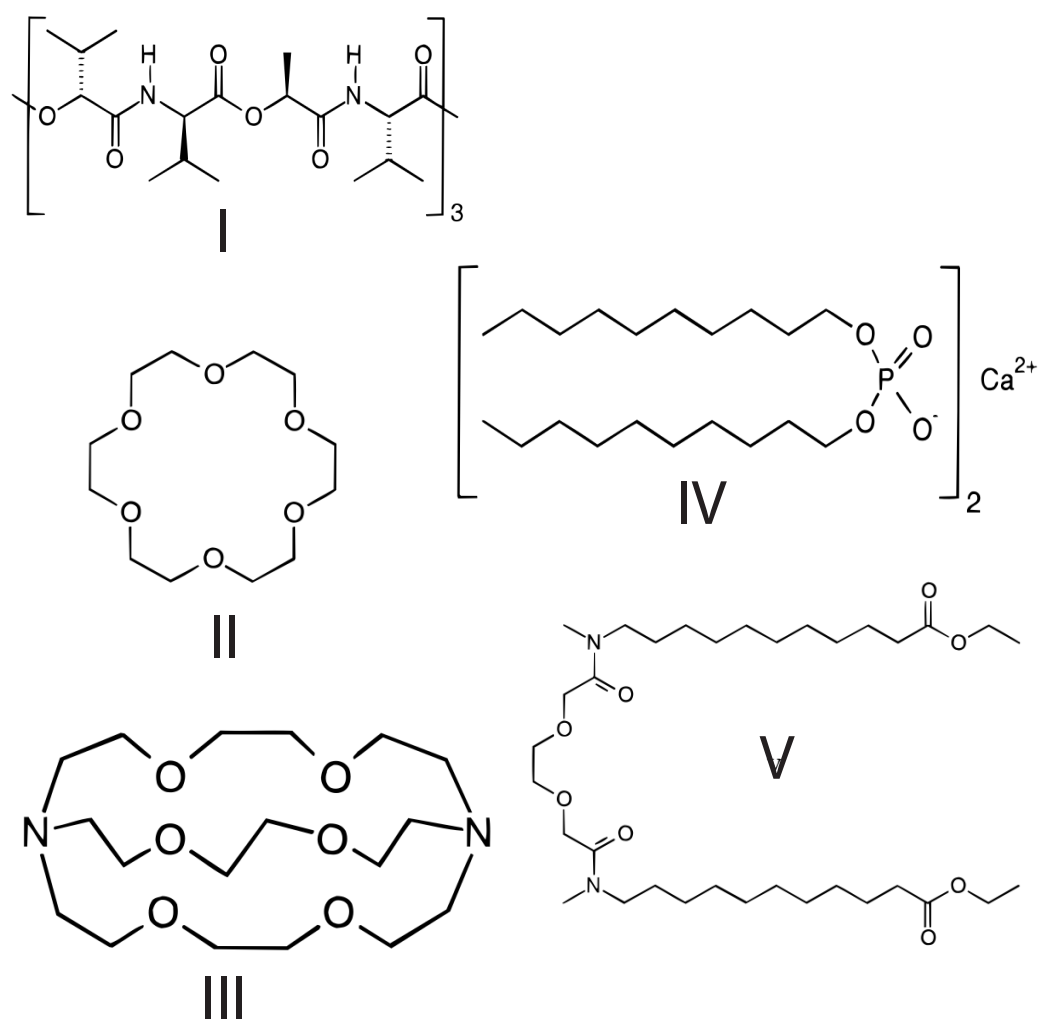

Figura 1.3. Estructuras de los primeros ionóforos selectivos a iones y compuestos relacionados: I, Valinomicina; II, 18-crown-6; III, criptando [2,2,2]; IV, dodecilfosfato de calcio; y V, el primer ionóforo lipofílico neutro selectivo a $\mathrm{Ca}^{2+}$.

La teoría para el mecanismo de respuesta de los ISE está muy bien establecida, sobre todo gracias al trabajo de Eisenman ${ }^{22}$. Formalmente, el potencial de membrana puede escribirse como la suma de los potenciales en las fases orgánica y acuosa y el potencial de difusión dentro de la membrana, siendo este último poco relevante en la práctica ${ }^{23,24,25}$. La selectividad en el intercambio de iones y la formación de complejos ahora se entienden bien, pero se necesitaron 
las últimas décadas para una mejor descripción de la respuesta de los ISE frente a soluciones que contienen iones con diferentes valencias.

Tabla 1.1. Algunos analitos para los que se han construido ESI. (*Modificado de E. Bakker, P. Buhlmann, E. Pretsch, Chem.Rev. 1997, 97, 3083-3132)

\begin{tabular}{ll}
\hline Grupo de analitos & Electrodos selectivos de iones \\
\hline Cationes inorgánicos & $\mathrm{H}^{+}, \mathrm{Li}^{+}, \mathrm{Na}^{+}, \mathrm{K}^{+}, \mathrm{Rb}^{+}, \mathrm{Cs}^{+}, \mathrm{Be}^{2+}, \mathrm{Mg}^{2+}, \mathrm{Ca}^{2+}, \mathrm{Sr}^{2+}, \mathrm{Ba}^{2+}, \mathrm{Mo}(\mathrm{IV})$, \\
& $\mathrm{Fe}(\mathrm{III}), \mathrm{Cu}(\mathrm{II}), \mathrm{Co}(\mathrm{II}), \mathrm{Ni}(\mathrm{II}), \mathrm{Ag}(\mathrm{I}), \mathrm{Zn}(\mathrm{II}), \mathrm{Cd}(\mathrm{II}), \mathrm{Hg}(\mathrm{II}), \mathrm{Tl}(\mathrm{I})$, \\
& $\mathrm{Bi}(\mathrm{III}), \mathrm{Al}(\mathrm{III}), \mathrm{Pb}(\mathrm{II}), \mathrm{U}(\mathrm{IV}), \mathrm{Sm}(\mathrm{II}), \mathrm{NH}_{4}^{+}$ \\
Aniones inorgánicos & $\mathrm{CO}_{3}{ }^{2-}, \mathrm{HCO}_{3}{ }^{-}, \mathrm{SCN}^{-}, \mathrm{NO}_{2}{ }^{-}, \mathrm{OH}^{-}, \mathrm{PO}_{4}{ }^{3-}, \mathrm{SO}_{3}{ }^{2-}, \mathrm{SO}_{4}{ }^{2-}, \mathrm{Cl}^{-}, \mathrm{SeO}_{3}{ }^{2-}, \mathrm{I}^{-}$ \\
Cationes orgánicos a & $\begin{array}{l}\text { 1-fenilamina, 1-(1-naftil)-etilamina, efedrina, norefedrina, } \\
\text { seudoefedrina, anfetamina, propanol, aminoácidos, bencilamina, } \\
\text { alquilaminas, dopamina, mexiletína, procaína, prilocaína, }\end{array}$ \\
& $\begin{array}{l}\text { lidocaína, bupivacaína, lignocaína, tetrametil y tetraetilamonio, } \\
\text { guanidina, merformina, fenformina, creatinina, protamina }\end{array}$ \\
Aniones orgánicos & $\begin{array}{l}\text { Salicilato, ftalato, maleato, 2-hidroxibencildroxamato, } \\
\text { nucleótidos, heparina }\end{array}$ \\
Analitos neutros & $\mathrm{CO}_{2}, \mathrm{NH}_{3}$ (indirectamente)
\end{tabular}

\footnotetext{
${ }^{a}$ Para los cationes que provienen de un compuesto neutro, se indica el nombre del compuesto del cual provienen.
} 


\section{MECANISMO DE RESPUESTA}

La teoría básica para la respuesta de los electrodos de membrana polimérica se desarrolló hace ya mucho tiempo. Sin embargo, la relevancia de las diversas contribuciones al potencial de membrana ha sido un tema muy debatido. Se reconoce plenamente que dichas membranas tienen propiedades intrínsecas de intercambio catiónico y por ello se han podido desarrollar modelos sencillos, de hecho se ha demostrado que las membranas basadas en ionóforos neutros con PVC como matriz polimérica y sin adición de sitios iónicos lipofílicos pueden dar respuesta catiónica sólo a causa de las impurezas presentes en la membrana ${ }^{26,27}$. Adicionalmente, en otros estudios las membranas que se construyeron con materiales rigurosamente purificados produjeron ESI que perdieron por completo su permeabilidad selectiva a cationes, incluso con valinomicina que es bastante selectiva ${ }^{28,29}$, lo que demuestra que la presencia de sitios iónicos es crucial para estos sensores.

Las membranas de los electrodos selectivos de iones suelen estudiarse en condiciones de corriente nula en una celda galvánica como la siguiente (ver figura 1.1):

Referencia externa I| muestra II membrana II solución interna || referencia interna

La fuerza electromotriz (fem) a través de esta celda es la suma de todas las contribuciones de los potenciales individuales. Muchos de estos potenciales son independiente, y la fem generalmente se puede escribir como: 


$$
\text { fem }=E_{\text {const }}+E_{J}+E_{M}
$$

donde $\mathbf{E}_{\mathbf{M}}$ es el potencial de membrana y $\mathbf{E}_{\mathbf{J}}$ es el potencial de unión líquida, que en condiciones bien definidas puede ser suficientemente pequeño y constante o también puede ser calculado de acuerdo con el formalismo de Henderson ${ }^{30}$. Es importante tener en cuenta que este potencial de unión líquida es el que prohíbe la verdadera evaluación de las actividades de los iones individuales con ISE, por lo tanto el papel que desempeña el electrodo de referencia debe tomarse muy en cuenta ${ }^{31}$.

\section{POTENCIALES LÍMITE}

La membrana por lo general está colocada entre la muestra y un electrolito de referencia interno, y con esto se divide el potencial de membrana en tres contribuciones separadas, primero los potenciales límites de fase en ambas interfaces y por último el potencial de difusión dentro de la membrana. Mientras que el potencial en la interfase membrana/solución interna por lo general se puede se puede suponer independiente de la muestra, el potencial de difusión dentro de la membrana puede ser relevante si surgen gradientes de concentración de iones. Sin embargo, evidencias experimentales han mostrado que el potencial de difusión es insignificante para la mayoría de los casos prácticos ${ }^{32,33}$, y que la permeabilidad selectiva de las membranas catiónicas basadas en PVC sin agregar aditivos iónicos se puede explicar por la presencia de impurezas aniónicas presentes en la matriz polimérica ${ }^{34,35}$. Por lo tanto, el modelo de potenciales límites de 
fase puede utilizarse para describir la respuesta de los electrodos selectivos de iones de forma muy precisa.

Para los ESI, el potencial de difusión dentro de la membrana es cero si no se producen gradientes de concentración. Este es el caso para las membranas que muestran una respuesta Nernstiana. Por simplicidad, los potenciales de difusión interna se tratan como efectos secundarios, y por lo tanto:

$$
E_{M}=E_{\text {const }}+E_{P B}
$$

donde $\mathrm{E}_{\mathrm{PB}}$ es el potencial límite en la interfase membrana/muestra.

En primer lugar, el potencial electroquímico, $\bar{\mu}$, se formula para la fase acuosa:

$$
\bar{\mu}_{(a c)}=\mu_{(a c)}+z F \phi_{(a c)}=\mu_{(a c)}^{0}+R T \ln a_{i(a c)}+z F \phi_{(a c)}
$$

y para la fase orgánica:

$$
\bar{\mu}_{(o r g)}=\mu_{(o r g)}+z F \phi_{(o r g)}=\mu_{(o r g)}^{0}+R T \ln a_{i(o r g)}+z F \phi_{(o r g)}
$$

donde $\boldsymbol{\mu}$ es el potencial químico ( $\boldsymbol{\mu}^{\mathbf{0}}$ en condiciones estándar), $\mathbf{z}$ es la valencia de ion y $\mathbf{a}_{\mathbf{i}}$ la actividad de ion "i" no complejado, $\boldsymbol{\phi}$ es el potencial eléctrico, y R, T y F son la constante 
universal de los gases, la temperatura absoluta y la constante de Faraday. Lo que se supone ahora es que los procesos de transferencia de iones y de complejación son relativamente rápidos y por lo tanto, el equilibrio se mantiene en la interfase, de manera que los potenciales electroquímicos son iguales para las dos fases. Esto conduce a la siguiente expresión para el potencial límite de fase:

$$
E_{P B}=\Delta \phi=-\frac{\mu_{(o r g)}^{0}-\mu_{(a c)}^{0}}{z F}+\frac{R T}{z F} \ln \frac{a_{i(a c)}}{a_{i(o r g)}}
$$

Siempre se espera una función simple para el potencial límite, siempre y cuando $\mathbf{a}_{\mathbf{i}(\mathbf{o r g})}$ no sea alterada significativamente por la muestra. Se ha demostrado que, diferentes membranas de PVC en contacto sólido se muestran como una función de la concentración del ionóforo, debido a la fuerte formación de complejos. En consecuencia, la concentración del complejo es aproximadamente igual a la de los sitios aniónicos y se altera si se añade un exceso de ionóforo. Entonces, la concentración del ionóforo en exceso es inversamente proporcional a la actividad de los cationes libres en la membrana. De a cuerdo con la ecuación 1.5, si se aumenta en 10 la concentración del ionóforo, esto aumenta el potencial en $\mathrm{RT} / \mathrm{zF}$, es decir, en $59.2 \mathrm{mV}$ para z=1 a 298 K. Este efecto no se puede detectar con electrodos selectivos de iones clásicos (ver figura 1) ya que el cambio en la actividad influye en el potencial de membrana y en la solución interna de manera simultánea. Por esto, se usó un electrodo de contacto interno sólido en donde la membrana polimérica se adhiere sobre el electrodo de referencia interno ${ }^{36}$.

Mediante la combinación de las ecuaciones 1.5 y 1.2 se obtiene:

$$
E_{M}=E_{c o n s t}+E_{P B}=E_{c o n s t}-\frac{\mu_{(o r g)}^{0}-\mu_{(a c)}^{0}}{z F}-\frac{R T}{z F} \ln a_{i(o r g)}+\frac{R T}{z F} \ln a_{i(a c)}
$$


y bajo la condición de que $\mathbf{a}_{\mathbf{i}(\text { org })}$ se mantenga constante, además de que las demás contribuciones sean independientes, la ecuación 1.6 se puede reducir a la famosa ecuación de Nernst:

$$
E_{M}=E^{0}+\frac{R T}{z F} \ln a_{i(a c)}
$$

De acuerdo con la ecuación 1.6, la composición de la superficie de la membrana que está en contacto con la muestra debe ser constante con el fin de obtener una respuesta Nernstiana para el electrodo. Sin embargo, si la ecuación 1.5 es válida, la estructura exacta de esta zona no es realmente relevante para la respuesta del sensor ${ }^{37}$. Para lograr una composición constante de la mayor parte de la membrana, se deben cumplir las siguientes condiciones:

(1) La membrana debe tener propiedades de intercambiador iónico. El equilibrio de coextracción simultánea de contraiones de la muestra se produce de acuerdo con la reacción: $I_{(a c)}^{+}+X_{(a c)}^{-} \Leftrightarrow I_{(\text {org })}^{+}+X_{(\text {org })}^{-}$. El principal factor que determina $\mathbf{a}_{\mathbf{i}(\mathbf{o r g})}$ es la presencia en la membrana de un intercambiador iónico lipofílico, ya que así la concentración de aniones extraídos $\mathbf{X}_{(\text {org) }}^{-}$es insignificante. Sin embargo, si la concentración del intercambiador iónico es pequeña en relación a la de $\mathbf{X}_{(\text {org) }}^{-}$, la concentración de iones primarios en la fase orgánica $\mathbf{a}_{\mathbf{i}(\mathbf{o r g})}$ es aproximadamente proporcional a la de $\mathbf{a}_{\mathbf{i}(\text { ac })}$ y el electrodo no responde al cambio de actividad del ion de manera Nernstiana.

(2) La membrana debe ser lo suficientemente hidrofóbica para no permitir demasiada coextracción de contraiones de acuerdo con el equilibrio de la condición 1. Esto permite la 
medición de muestras con altas concentraciones de electrolito. Por esto, polímeros hidrofílicos tales como hidrogeles no son adecuados como materiales para la construcción de electrodos selectivos de iones.

(3) Si se producen reacciones de intercambio iónico con iones interferentes de la misma carga, la actividad $\mathrm{a}_{\mathrm{i}(\text { org }}$ del ion primario no complejado se reduce en la ecuación 1.7 y se espera una respuesta sub-Nernstiana. Esto se puede prevenir mediante la incorporación en la membrana de un ionóforo lipofílico que se una selectivamente al ion primario.

(4) Sin embargo, el ionóforo no debe unirse al ion primario con demasiada fuerza.

(5) No deben estar presentes en la muestra otras especies (eléctricamente neutras) interferentes que se puedan extraer a la membrana y alterar la actividad $\mathrm{a}_{\mathrm{i}(\mathrm{org})}$.

\section{SELECTIVIDAD}

La selectividad es claramente una de las características más importantes de un sensor, ya que a menudo determina si una medición es fiable. Es especialmente crítica en aplicaciones clínicas donde el error para las mediciones en sangre o suero no puede ser mayor que $0.1 \mathrm{mV}$. Un buen estudio de selectividad, en teoría permite a los investigadores identificar los parámetros clave para la optimización del rendimiento de los sensores potenciométricos, por ejemplo, mediante el ajuste de los parámetros (es decir, las concentraciones de los componentes de la membrana) o elegir diferentes plastificantes o matrices. 
Los electrodos selectivos de iones, con solo el hecho de recibir el calificativo de selectivo y no de específicos, ya estamos diciendo que estos electrodos pueden presentar alguna señal a iones diferentes al primario. A veces, la presencia de ciertos iones en la solución de la especie a determinar con los electrodos selectivos producen interferencias en la medida del ion para el que está diseñado. Estas interferencias pueden ser de naturaleza aniónica o catiónica, analicemos cada caso.

\section{INTERFERENCIAS CATIÓNICAS}

Supongamos un electrodo selectivo constituido por una membrana tipo sulfuro, tal como el hipotético MeS. En presencia de ion interferente, $\mathrm{Int}^{2+}$, se tendrá la reacción:

$$
M e S+\operatorname{Int}{ }^{2+} \leftrightarrow \operatorname{Int} S+M e^{2+}
$$

Por lo que al pasar iones $\mathrm{Me}^{2+}$ desde la membrana a la solución, se tendrá en esta una concentración superior a la existente antes de producirse el fenómeno de interferencia, es decir:

$$
\left[M e^{2+}\right]_{\text {Total }}=\left[M e^{2+}\right]_{\text {Añadido }}+\left[M e^{2+}\right]_{\text {Interferente }}
$$

Por otra parte la constante de la reacción interferente será $\mathrm{K}_{\mathrm{r}}$, que puede ser expresada como: 


$$
K_{r}=\frac{\left[\left[\mathrm{Me}^{2+}\right]_{\text {Interferente }}\left[\mathrm{S}^{2-}\right]\right]}{\left[\text { Int }{ }^{2+}\right]\left[S^{2-}\right]}=\frac{K_{s(M e S)}}{K_{s(I n t S)}}=K_{M e^{2+}, I n t^{2+}}^{P o t}
$$

De donde $\left[\mathrm{Me}^{2+}\right]_{\text {Interferente }}$ responde a la expresión:

$$
\left[M e^{2+}\right]_{\text {Interferente }}=\frac{K_{s(M e S)}}{K_{s(I n t S)}}\left[\operatorname{Int} t^{2+}\right]=K_{M e^{2+}, I n t^{2+}}^{P o t}\left[\operatorname{Int} t^{2+}\right]
$$

Siendo $K_{\mathrm{Me}^{2+}, \mathrm{Int}^{2+}}^{\mathrm{Pot}}$ el coeficiente de selectividad potenciométrico. Al aplicar la ecuación de Nernst, se tendrá la siguiente expresión:

$$
E=E^{o}+\frac{R T}{2 F} \ln \left(\left[M e^{2+}\right]_{\text {Añadido }}+K_{M e^{2+}, I n t^{2+}}^{P o t}\left[\operatorname{Int}{ }^{2+}\right]\right)
$$

a esta ecuación se le denomina ecuación de Nikolsky- Eisenman.

\section{INTERFERENCIAS ANIÓNICAS}

Determinados aniones pueden formar precipitados con los cationes constitutivos de las membranas selectivas de iones, pudiendo producir interferencias de tipo aniónico. Supongamos un electrodo cuya membrana selectiva este formada por una sal poco soluble de un halogenuro de $\operatorname{Ag}(\mathrm{I})$, tal como $\mathrm{AgX}$, en presencia de un anión interferente tal como $\mathrm{Y}^{\mathrm{n}-}$, con el que reacciona según el siguiente equilibrio de intercambio:

$$
n A g X_{(s)}+Y^{n-} \leftrightarrow A g_{n} Y_{(s)}+n Y_{I n t}^{-}
$$


O bien:

$$
A g X_{(s)}+\frac{1}{n} Y^{n-} \leftrightarrow A g Y_{1 / n}+X_{I n t}^{-}
$$

Regido por la constante:

$$
K_{r}=\frac{\left[X^{-}\right]_{I n t}\left[A g^{+}\right]}{\left[Y^{n-}\right]^{1 / n}\left[A g^{+}\right]}=\frac{K_{s(A g X)}}{\left(K_{s\left(A g_{n} Y_{(s)}\right)}\right)^{1 / n}}
$$

De donde, despejando $\left[X^{-}\right]_{I n t}$, se tiene:

$$
\left[X^{-}\right]_{I n t}=\frac{K_{s(A g X)}}{\left(K_{s\left(A g_{n} Y_{(s)}\right)}\right)^{1 / n}}\left[Y^{n-}\right]^{1 / n}=K_{X^{-}, Y^{n-}}^{P o t}\left[Y^{n-}\right]^{1 / n}
$$

y el potencial de celda será finalmente:

$$
E=E^{o}+\frac{R T}{F} \ln \left(\left[X^{-}\right]_{\text {Añadido }}+K_{X^{-}, Y^{n-}}^{\text {Pot }}\left[Y^{n-}\right]^{1 / n}\right)
$$

de manera general, la ecuación de Nikolskii se describe ahora, teniendo en cuenta las posibles interferencias aniónicas y catiónicas, como sigue:

$$
E=E^{o}+\frac{R T}{z_{i} F} \ln \left(a_{i}+\sum_{j=1}^{n} K_{i, j}^{P o t} a_{j}^{z_{i} / z_{j}}\right)
$$

donde i y j se refieren a la especie primaria (ion para el que sea construido el electrodo) y a las especies interferentes respectivamente, deduciéndose por tanto que cuanto menor sea el valor de $K_{i, j}^{\text {Pot }}$ más selectivo será el electrodo y menos importante la interferencia, ya que si el valor de las 
constantes de interferencia se aproxima a cero, el término de la suma tambien se aproxima a cero, lo que nos llevaría a la ecuación de Nernst para un electrodo selectivo ideal.

Como se puede deducir en la ecuación de Nikolskii entre más grande sea la constante potenciométrica, mayor será la interferencia. Por ejemplo, si $K_{i, j}^{P o t}>1$ entonces esto implicaría que la interferencia es demasiado grande y el ISE no es selectivo hacia el que se pensaba ion primario, si $K_{i, j}^{\text {Pot }}=1$ entonces el ISE responde de igual manera hacia el ion principal como al ion interferente y si $K_{i, j}^{P o t}<1$ esto implicaría que el sensor es selectivo al ion principal y entre más cercana a cero sea esta constante más selectivo será el ESI y menos importante la interferencia.

Hablemos ahora de cómo calcular experimentalmente las constantes de selectividad. La IUPAC recomienda dos métodos para el cálculo de estos parámetros, el método de las soluciones separadas y el método de interferencia fija, que son de gran importancia para los ESI, ya que el valor de estas constantes determina el grado de afinidad que iones interferentes presentan frente al ion principal.

\section{MÉTODO DE LAS SOLUCIONES SEPARADAS}

Hay que advertir que los electrodos selectivos responden primariamente al ion para el que han sido construidos (ion primario i) y secundariamente a los iones interferentes (ion interferente j). El método de las soluciones separadas consiste en preparar una serie de soluciones del ion primario de concentración creciente y otra serie de soluciones del ion interferente de concentración creciente. Si se trata de un electrodo selectivo a cationes, al representar el 
potencial (E) para el ion primario e interferente en el mismo diagrama E Vs log (a), se tendrá algo parecido a la Figura 1.4.

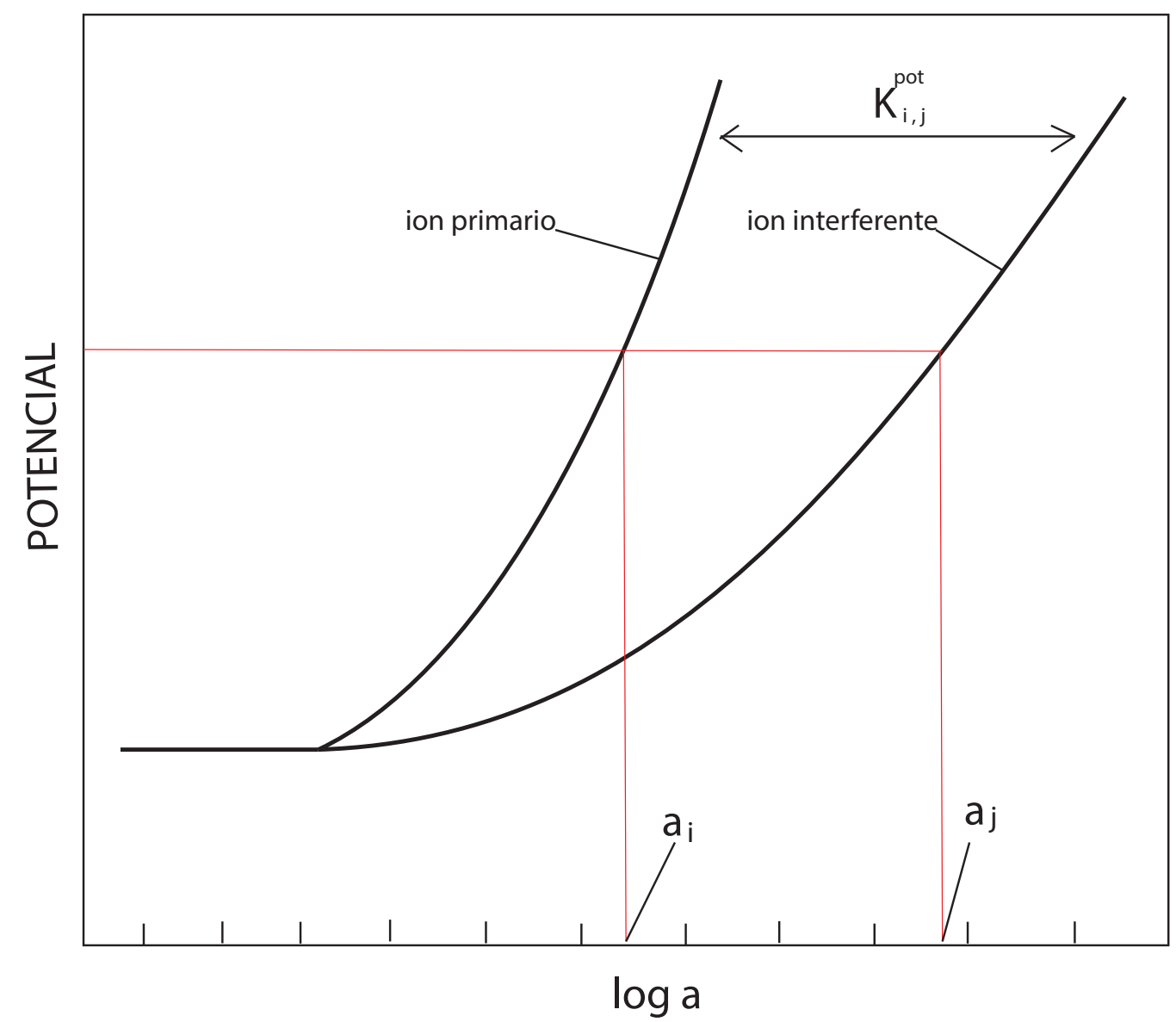

Figura 1.4. Método de las soluciones separadas empleando en el cálculo de $K_{i, j}^{P o t} \quad$ (igualando potenciales).

Las expresiones del potencial para cada uno de los iones serán las siguientes:

Para el ion principal: $\quad E_{i}=E^{o}+\frac{R T}{z_{i} F} \ln a_{i}$ 
Para el ion interferente: $\quad E_{j}=E^{o}+\frac{R T}{z_{i} F} \ln \left(K_{i, j}^{\text {pot }} a_{j}^{z_{i} / z_{j}}\right)$

En estas expresiones se observa además que tanto en una como en otra la pendiente de la recta es la misma, ya que el electrodo está construido para responder al ion i-ésimo o primario, a partir de la Figura 1.4 puede considerarse el mismo potencial para ambos iones, obteniéndose, al igualar dichos potenciales, la expresión:

$$
E^{o}+\frac{R T}{z_{i} F} \ln a_{i}=E^{o}+\frac{R T}{z_{i} F} \ln \left(K_{i, j}^{p o t} a_{j}^{z_{i} / z_{j}}\right)
$$

sumando $-E^{o}$ de ambos lados de la ecuación, se obtiene

$$
\frac{R T}{z_{i} F} \ln a_{i}=\frac{R T}{z_{i} F} \ln \left(K_{i, j}^{p o t} a_{j}^{z_{i} / z_{j}}\right)
$$

ahora, si multiplicamos la ecuación por el factor $\frac{z_{i} F}{R T}$, tenemos que

$$
\ln a_{i}=\ln \left(K_{i, j}^{p o t} a_{j}^{z_{i} / z_{j}}\right)
$$

aplicando la inversa de la función logaritmo natural, tenemos

$$
a_{i}=\left(K_{i, j}^{p o t} a_{j}^{z_{i} / z_{j}}\right)
$$


y por tanto:

$$
K_{i, j}^{p o t}=\frac{a_{i}}{a_{j}^{z_{i} / z_{j}}}
$$

Donde todo es conocido, pudiendo medirse el coeficiente de selectividad muy fácilmente. Ahora bien, si se considera una misma actividad, a, para ambos iones, resultarán dos potenciales diferentes que responden a las siguientes expresiones:

Para el ion principal: $E_{i}=E^{o}+\frac{R T}{z_{i} F} \ln a$

Para el ion interferente: $E_{j}=E^{o}+\frac{R T}{z_{i} F} \ln \left(K_{i, j}^{p o t} a^{z_{i}} z_{z_{j}}\right)$

Y haciendo $E_{i}-E_{j}$ se obtiene que

$$
\begin{gathered}
\Delta E_{i, j}=E_{i}-E_{j}=\frac{R T}{z_{i} F} \ln a-\frac{R T}{z_{i} F} \ln \left(K_{i, j}^{p o t} a^{z_{i}} z_{j}\right)=\frac{R T}{z_{i} F}\left[\ln a-\ln \left(K_{i, j}^{p o t} a^{z_{i} / z_{j}}\right)\right] \\
\Rightarrow \Delta E_{i, j}=\frac{R T}{z_{i} F} \ln \left(\frac{a}{K_{i, j}^{p o t} a^{z_{i} / z_{j}}}\right)
\end{gathered}
$$

despejando $K_{i, j}^{P o t}$

$$
K_{i, j}^{p o t}=\frac{a^{\left(1-\frac{z_{i}}{z_{j}}\right)}}{10^{z_{i} F \frac{\Delta E_{i, j}}{R T}}}
$$

Donde $\Delta E_{i, j}$ y $a$ son datos experimentales y los demás parámetros son conocidos. 
Entonces, en general el método de las soluciones separadas consiste en construir curvas de calibración potenciométricas para el ion principal y para cada uno de los posibles iones interferentes por separado, y basta con elegir un potencial o una actividad constante para calcular fácilmente los coeficientes de selectividad, es importante mencionar que el potencial o la actividad que elijamos debe estar necesariamente dentro de la parte lineal de la curva de calibración, es decir, donde la respuesta del ESI este regida por la ecuación de Nernst, ya que en caso contrario todas las ecuaciones anteriores no se cumplen.

\section{MÉTODO DE LAS SOLUCIONES MEZCLADAS}

En este método se miden los potenciales de soluciones que contienen concentraciones variables del ion primario y una concentración fija y conocida del interferente, $\mathbf{a}_{\mathrm{j}}$, obteniéndose la curva de calibrado que se muestra a continuación.

Se considera la zona III, en el que predomina la intervención del ion primario, la zona II, en el que intervienen tanto el ion primario como el interferente, y, finalmente, la zona I, en la que predomina la intervención del ion interferente. Trazando las correspondientes tangentes, se observa un punto de intersección a una actividad, $\mathrm{a}_{\mathrm{i}}$, a partir de la cual se determina el coeficiente de selectividad potenciométrica del electrodo, ya que, como se muestra en el caso de las soluciones separadas, considerando el mismo valor de potencial, se calcula dicha constante, expresión en la que se conoce $\mathbf{a}_{\mathrm{j}}$ y se mide gráficamente $\mathbf{a}_{\mathrm{i}}$. 


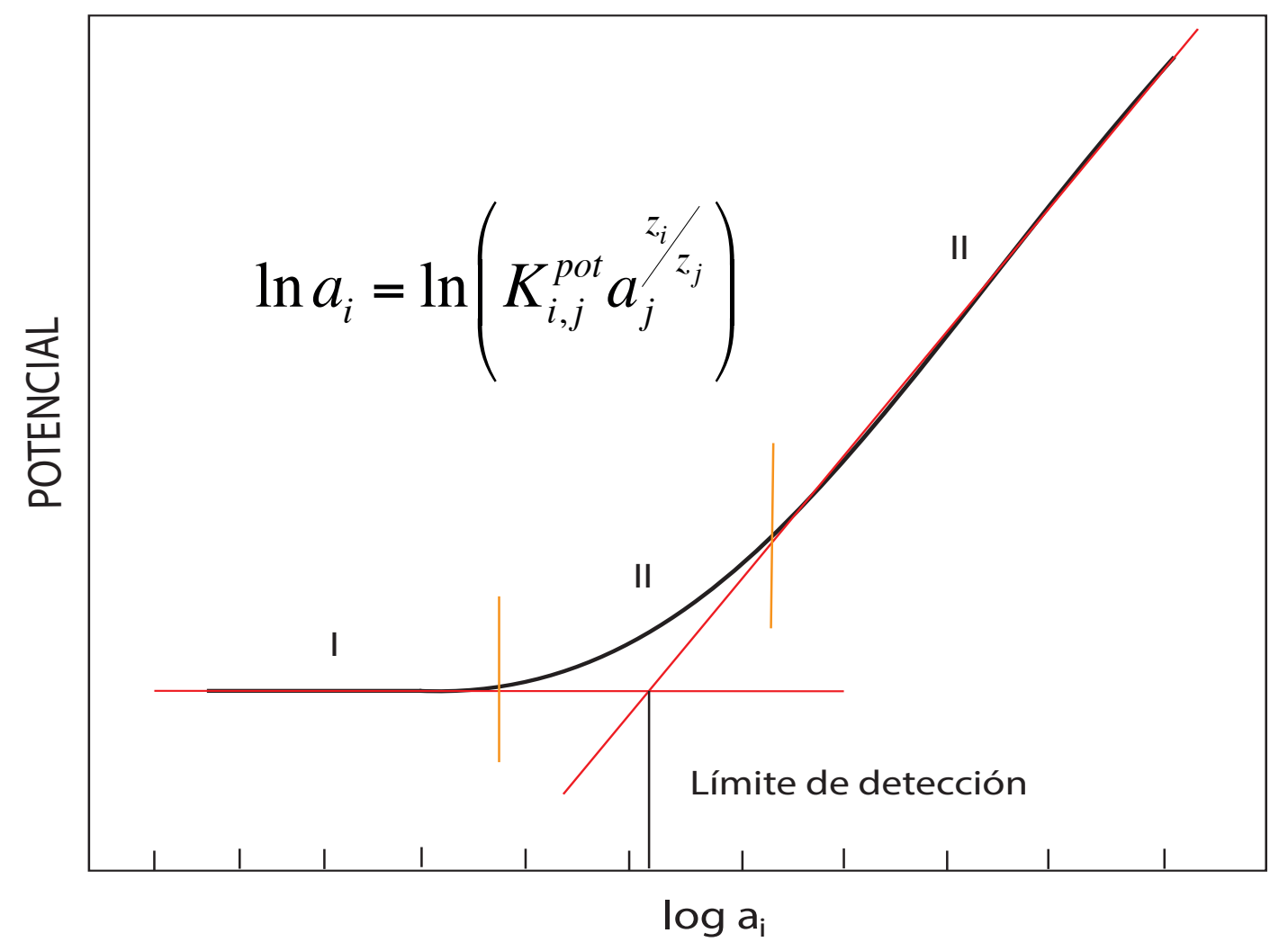

Figura 1.5. Método de las disoluciones mezcladas empleado en la medida de $K_{i, j}^{\text {pot }}$

Se considera la zona III, en el que predomina la intervención del ion primario, la zona II, en el que intervienen tanto el ion primario como el interferente, y, finalmente, la zona I, en la que predomina la intervención del ion interferente. Trazando las correspondientes tangentes, se observa un punto de intersección a una actividad, $\mathrm{a}_{\mathrm{i}}$, a partir de la cual se determina el coeficiente de selectividad potenciométrica del electrodo, ya que, como se muestra en el caso de las soluciones separadas, considerando el mismo valor de potencial, se calcula dicha constante, expresión en la que se conoce $\mathbf{a}_{\mathbf{j}}$ y se mide gráficamente $\mathbf{a}_{\mathbf{i}}$. 


\section{LÍMITE DE DETECCIÓN}

En los últimos años, la potenciometría ha sido utilizada en "análisis traza" y se utiliza como remplazo de técnicas como voltamperometría, espectroscopía, absorción atómica, e incluso de espectroscopía de masas de plasma (ICP/MS) ${ }^{38}$. Hoy en día, los ISE optimizados pueden alcanzar límites de detección bastante bajos, se han encontrado en revistas especializadas reportes de ESI con límites de detección a niveles picomolares $\left(10^{-12} \mathrm{~mol} \mathrm{~L}^{-1}\right)$, cifra sorprendente cuando se comparan los límites de detección que se alcanzan con técnicas bien establecidas y además costosas por ejemplo la electroforesis capilar y las antes mencionadas.

Un gráfico de calibración tiene normalmente la forma mostrada en la Figura 1.6, utilizando las condiciones experimentales para la determinación del límite de detección recomendadas por la IUPAC $^{39}$, se muestra la respuesta potenciométrica de un ESI hipotético que responde al catión $\mathrm{M}^{z+}$. En la curva se distinguen tres zonas: la zona A, es la parte donde el potencial del ESI permanece constante al aumento en la concentración del catión; la zona B, es donde el ESI responde selectivamente al catión $\mathrm{M}^{z+}$ y la pendiente está regida por la ecuación de Nernst y la Zona C, donde en ISE deja de responder a la concentración del analito. En la figura resaltan dos puntos etiquetados como I y II que se definen como el límite de detección inferior y el límite de detección superior respectivamente, para fines prácticos se pondrá atención solamente en el punto I, el punto II puede ser descrito de manera similar.

Por analogía con las definiciones adoptadas en otros campos, un límite de detección podría ser definido como la concentración para la cual, en las condiciones especificadas, el potencial de el 
ESI, E, se desvía del potencial promedio en la región A por un múltiplo del error estándar de una sola medida del potencial de electrodo en esta región A. El múltiplo seleccionado dependerá del nivel de significación estadística seleccionado.

La diferencia fundamental en la naturaleza de los ESI (su respuesta logarítmica) en comparación con los métodos lineales, justifica otro tipo de definición del límite de detección. Por motivos de conveniencia práctica, se recomienda una definición más simple. El límite de detección puede ser tomado como la actividad (o la concentración) del catión $\mathrm{M}^{\mathrm{z+}}$ en el punto de intersección de las curvas de extrapolación de las zonas A y B, como se muestra en la figura 1.6.

Ahora, ¿qué tan confiable es este método? la respuesta a esta pregunta depende de:

1. La desviación estándar de una sola medición del potencial en ambas regiones lineales (que no tiene por qué ser la misma en la Nernstiana y en regiones independientes de la concentración);

2. El número de puntos experimentales tomados en cuenta para determinar los parámetros de las zonas lineales;

3. El intervalo de concentraciones utilizado para determinar los parámetros de las zonas lineales.

Con base en lo anterior, además de las condiciones experimentales (es decir, la composición de la solución, la forma de construcción y de preacondicionamiento del electrodo, velocidad de agitación, etc) utilizados para la determinación del límite de detección, los detalles de los datos experimentales, (por ejemplo, el número de mediciones, la desviación estándar de una sola 
medición, etc) deben ser ser reportados. Sin embargo, la descripción de las condiciones experimentales y el método de evaluación sólo ofrece la posibilidad de reproducir los datos, pero no ayuda en la comparación de los datos obtenidos en diferentes condiciones ${ }^{40}$.

En consecuencia, las condiciones experimentales y el método de evaluación deben estandarizarse, por ejemplo:

1. Los potenciales empleados para la determinación del límite de detección deben medirse como mínimo por triplicado.

2. Los datos utilizados para determinar las zonas lineales deben tomarse en un intervalo de concentraciones de dos órdenes de magnitud mayor, y dos órdenes de magnitud menor, incluyendo la concentración del punto de cruce. Para determinar las zonas lineales se deben tomar en cuenta un mínimo de cinco puntos de datos en ambas regiones.

3. Los parámetros de las zonas lineales determinadas deben indicarse con sus desviaciones estándar.

4. Con base en lo anterior, junto a los valores más probables de los límites de detección, también se debe reportar el intervalo de confianza.

5. Si la determinación de datos por debajo del límite de detección es problemática, (tiempo de respuesta demasiado largo, mala reproducibilidad, etc) se debe trazar una línea paralela al eje x en base al valor promedio del potencial medido para la solución de menor concentración. El límite de detección debe reportarse de manera similar a lo dicho anteriormente (valor central \pm desviación estándar) 


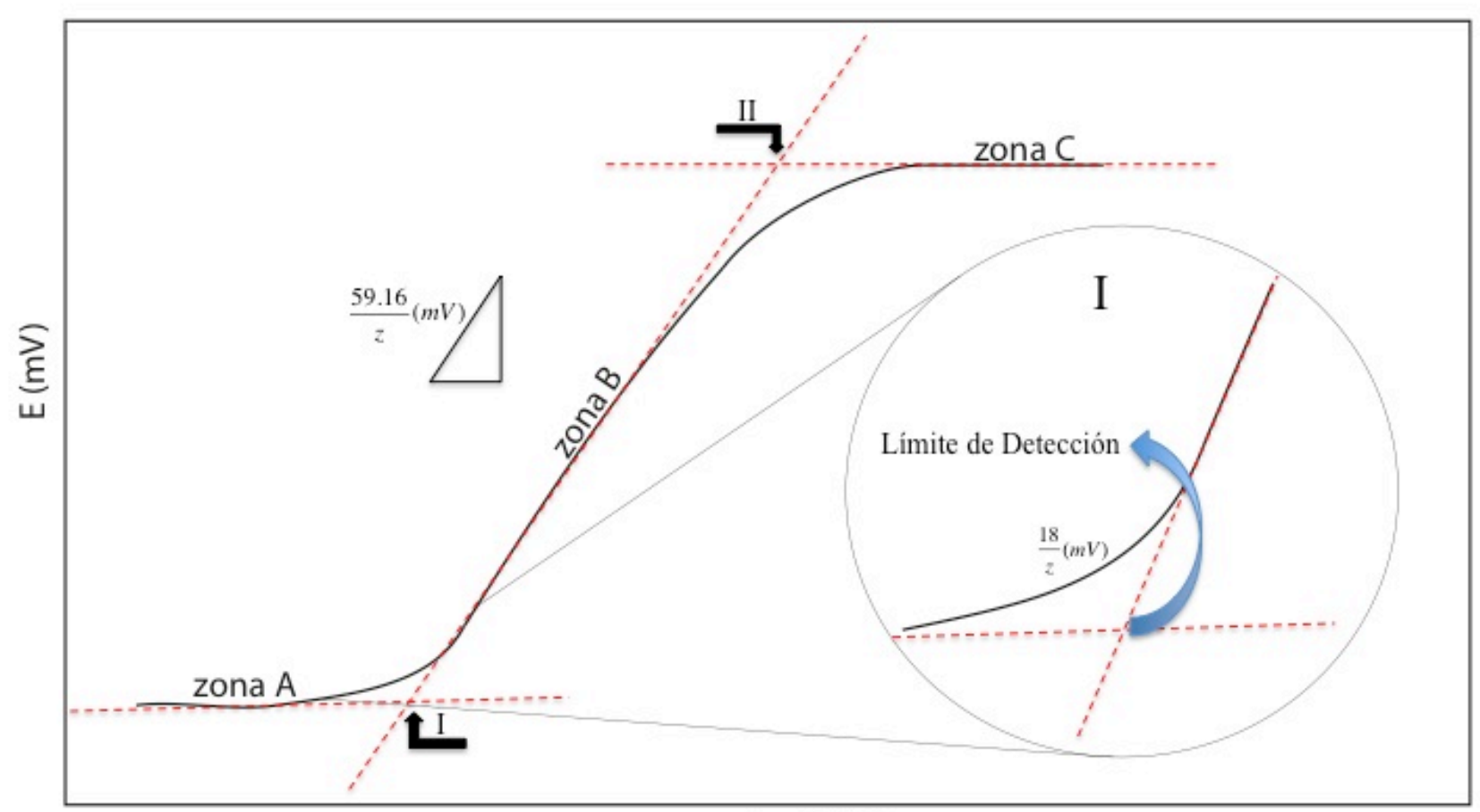

$\log \left[\mathrm{M}^{2+}\right]$

Figura 1.6. Respuesta potenciométrica de un ISE hipotético, debida al aumento en la concentración de iones $\mathrm{M}^{z+}$. La zona A, es la parte donde el potencial del ESI permanece constante al aumento en la concentración del catión; la zona B, es donde el ESI responde selectivamente al catión $\mathrm{M}^{\mathrm{z+}}$ y la pendiente esta regida por la ecuación de Nernst y la Zona C, donde en ESI deja de responder a la concentración del analito. Los puntos I y II representan los límites de detección inferior y superior respectivamente. En el círculo se hace un acercamiento del límite de detección inferior, donde se observa mejor el punto de cruce de las líneas extrapoladas (zona A y B) definido como el límite de detección de la técnica.

Los factores que afectan los límites de detección en los ESI son los gradientes de concentración a través de la membrana sensora y los flujos de iones interferentes en la membrana, estos factores se pueden minimizar con la optimización y acondicionamiento de la 
membrana. La disminución del flujo de iones por la membrana sensora es beneficioso para llegar a los niveles más bajos posibles en el límite de detección ${ }^{41,42}$. Hoy en día se pueden diseñar los ESI con membranas con: ionóforos inmovilizados, plastificantes y sitios iónicos; un aumento del contenido polimérico para disminuir los coeficientes de difusión; aumento del espesor de la membrana; disminución de la concentración de sitios iónicos; disminución de la superficie activa; e incorporación de partículas lipofílicas.

La agitación intensa puede quitar los rastros de contaminación de iones en las superficies de los ESI, que no pudieron minimizarse en la etapa de optimización y acondicionamiento. De hecho, los mejores límites de detección se alcanzaron en soluciones agitadas vigorosamente. ${ }^{43,44,}$ 45

Por último, se ha reportado que con el uso de electrodos de contacto sólido se pueden eliminar en gran medida los flujos de iones en las membranas sensoras $46,47,48,49,50,51,52$. En consecuencia, los electrodos de contacto sólido en combinación con la miniaturización del sensor son los candidatos para mejorar los límites de detección en varios órdenes de magnitud.

Mediante la combinación de varias de esta estrategias, límites de detección en niveles nano e incluso picomolares por litro se han reportado para gran variedad de ESI, y se ha demostrado que los ESI son adecuados para la determinación de iones metálicos en aguas ${ }^{53,54,55}$. Estos logros no serían posibles sin la mejora simultánea de los coeficientes de selectividad de los ESI. 


\section{ELECTRODOS SELECTIVOS AL MERCURIO}

Muchos electrodos selectivos a $\mathrm{Hg}$ (II) basados en ionóforos cargados se han reportado. La ditizona $\left(\mathbf{H g}^{2+} \mathbf{- 1}\right)$, por ejemplo, ha sido utilizada para la construcción de electrodos selectivos a mercurio ${ }^{56}$. Un ISE para mercurio basado en dicetohidrindileno-dicetohidramina $\left(\mathbf{H g}^{\mathbf{2 +}} \mathbf{- 2}\right)$ presentó una alta discriminación a iones de metales de transición excepto a plata $\left(\log K_{H g, j}^{p o t}: \mathrm{Ag}^{+}\right.$, $\left.+0.3 ; \mathrm{Zn}^{2+},-3.0 ; \mathrm{Pb}^{2+},-3.4 ; \mathrm{Cu}^{2+},-1.9\right){ }^{57}$. Para el caso del ionóforo $\mathrm{N}$-(tiofosforil)tiobenzamida $\left(\mathbf{H g}^{2+} \mathbf{- 3}\right)$ se obtuvo una discriminación para metales de transición, pero otra vez se observó interferencia de la plata $\left(\log K_{H g, j}^{\text {pot }}: \mathrm{Ag}^{+}, 0.0 ; \mathrm{Zn}^{2+},-2.9 ; \mathrm{Pb}^{2+},-2.6 ; \mathrm{Cu}^{2+},-2.7 ; \mathrm{Fe}^{3+},-3.4\right){ }^{58}$.<smiles>S=C(N=Nc1ccccc1)NNc1ccccc1</smiles><smiles>O=C1c2ccccc2C(=O)C1N=c1c(=O)c2ccccc2c1=O</smiles><smiles>CC(C)COP(=S)(NC(=S)c1ccccc1)OCC(C)C</smiles>

$$
\mathrm{Hg}^{2+}-3
$$


Por otro lado, también se han reportado ionóforos neutros en la construcción de ESI para mercurio. Una buena discriminación para $\mathrm{Fe}^{3+} \mathrm{y} \mathrm{Ag}^{+}$se obtuvo con el ionóforo 1,4-ditio-12corona-4 $\left(\mathrm{Hg}^{2+}-4\right)\left(\log K_{H g, j}^{p o t}: \mathrm{Ag}^{+},-3.4 ; \mathrm{Zn}^{2+},<-5.0 ; \mathrm{Pb}^{2+},-3.6 ; \mathrm{Cu}^{2+},-3.1 ; \mathrm{Fe}^{3+},-1.5 ; \mathrm{Na}^{+},-2.8\right)$ ${ }^{59}$. El ion con mayor interferencia para un electrodo basado en hexatiociclooctadecano $\left(\mathbf{H g}^{2+} \mathbf{- 5}\right)$

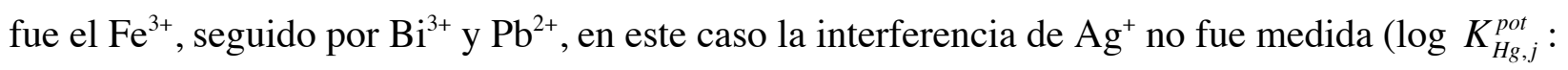
$\left.\mathrm{Fe}^{3+},+0.7 ; \mathrm{Bi}^{3+},+0.1 ; \mathrm{Pb}^{2+},-0.8\right){ }^{60}$. Dos ionóforos $\mathrm{N}, \mathrm{N}$ '-sustituidos 1,10 -diaza-18 corona-6 éteres $\left(\mathbf{H g}^{2+}-\mathbf{6}\right.$ y $\mathbf{H g}^{2+}$-7) mostraron una alta discriminación hacia diferentes cationes, pero una severa interferencia para $\mathrm{Ag}^{+}\left(\log K_{H g, j}^{\text {pot }}: \mathrm{Ag}^{+},+2.6 ; \mathrm{Zn}^{2+},-3.5 ; \mathrm{Pb}^{2+},-3.7 ; \mathrm{Na}^{+},-5.3 ; \mathrm{Cd}^{2+},-3.9\right){ }^{61}$, 62.

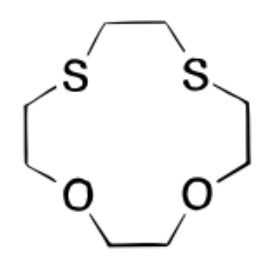

$\mathrm{Hg}^{2+}-4$<smiles>C1CSCCSCCSCCSCCS1</smiles>

$\mathrm{Hg}^{2+}-5$<smiles>[R]N1CCOCCOCCN(P)CCOCCO1</smiles><smiles>Cc1ccc2cc(P=[Pb])ccc2n1</smiles> 
Hasta ahora se han expuesto algunos de los ISE selectivos a $\mathrm{Hg}(\mathrm{II})$ más significativos hasta la década de los 90, pero más recientemente también se han propuesto bastantes membranas selectivas a mercurio pero ahora haciendo uso de la arquitectura molecular para el diseño de los ionóforos. Por ejemplo, un ESI basado en p-ter-butil calix[4] corona $\left(\mathbf{H g}^{\mathbf{2 +}} \mathbf{- 8}\right)$ mostró buena discriminación para algunos catones, pero una fuerte interferencia de $\mathrm{Ag}^{+}\left(\log K_{H g, j}^{p o t}: \mathrm{Ni}^{2+},-3.4\right.$; $\left.\mathrm{Zn}^{2+},-3.8 ; \mathrm{Pb}^{2+},-3.3 ; \mathrm{Cd}^{2+},-3.6\right){ }^{63}$. Por su parte, la membrana selectiva basada en el ionóforo macrocíclico ditiofosforado $\left(\mathbf{H g}^{2+} \mathbf{- 9}\right)$ mostró la siguiente selectividad (log $K_{H g, j}^{p o t}: \mathrm{Ag}^{+},-0.7 ; \mathrm{Ni}^{2+}$, -2.4; $\left.\mathrm{Co}^{2+},-2.2 ; \mathrm{Pb}^{2+},-1.2 ; \mathrm{Cd}^{2+},-2.2\right)^{64}$. El ESI basado en el receptor catiónico 5,11,17,23,tetra, tert, butil-25,27-dihidroxi-26,28-bis(O-metil glicilcarbonilmetoxi) tiocalix [4]-areno $\left(\mathbf{H g}^{\mathbf{2 +}} \mathbf{- 1 0}\right)$ presentó límites de detección alrededor de $10^{-8} \mathrm{~mol} \mathrm{~L}^{-1}$ y una alta discriminación hacia los iones estudiados $\left(\log K_{H g, j}^{p o t}: \mathrm{Ag}^{+},-2.9 ; \mathrm{Ni}^{2+},-3.5 ; \mathrm{Co}^{2+},-5.1 ; \mathrm{Cd}^{2+},-3.3\right){ }^{65}$.
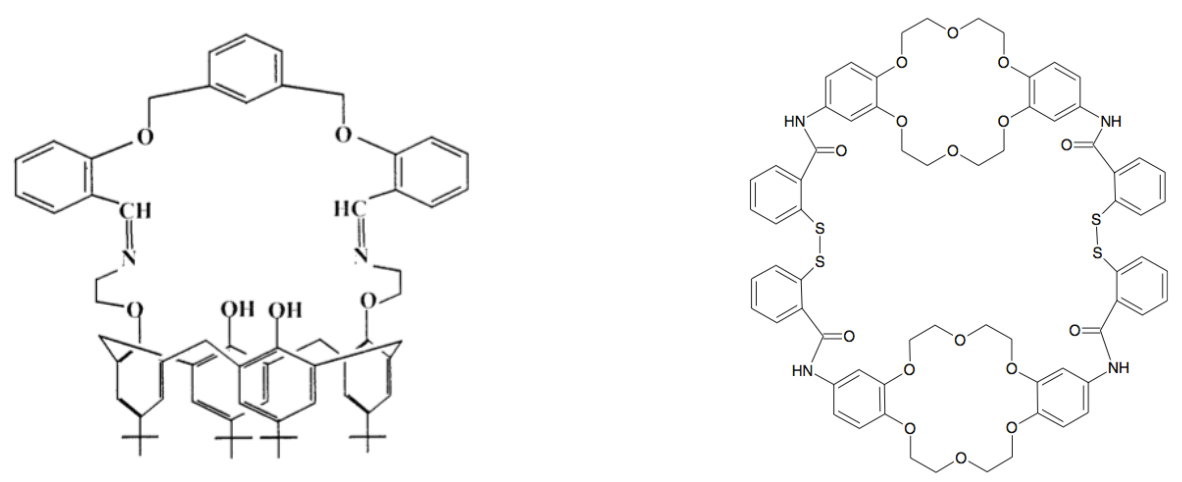

$\left(\mathrm{Hg}^{2+}-8\right)$

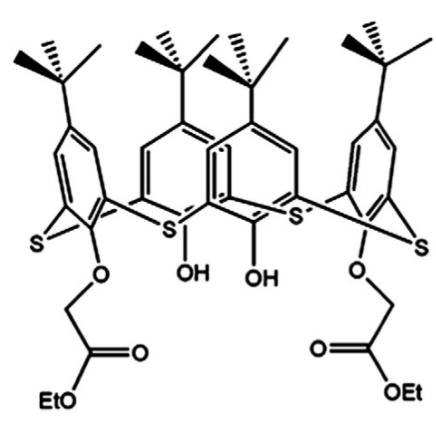

$\left(\mathrm{Hg}^{2+}-9\right)$

$\left(\mathrm{Hg}^{2+}-10\right)$ 


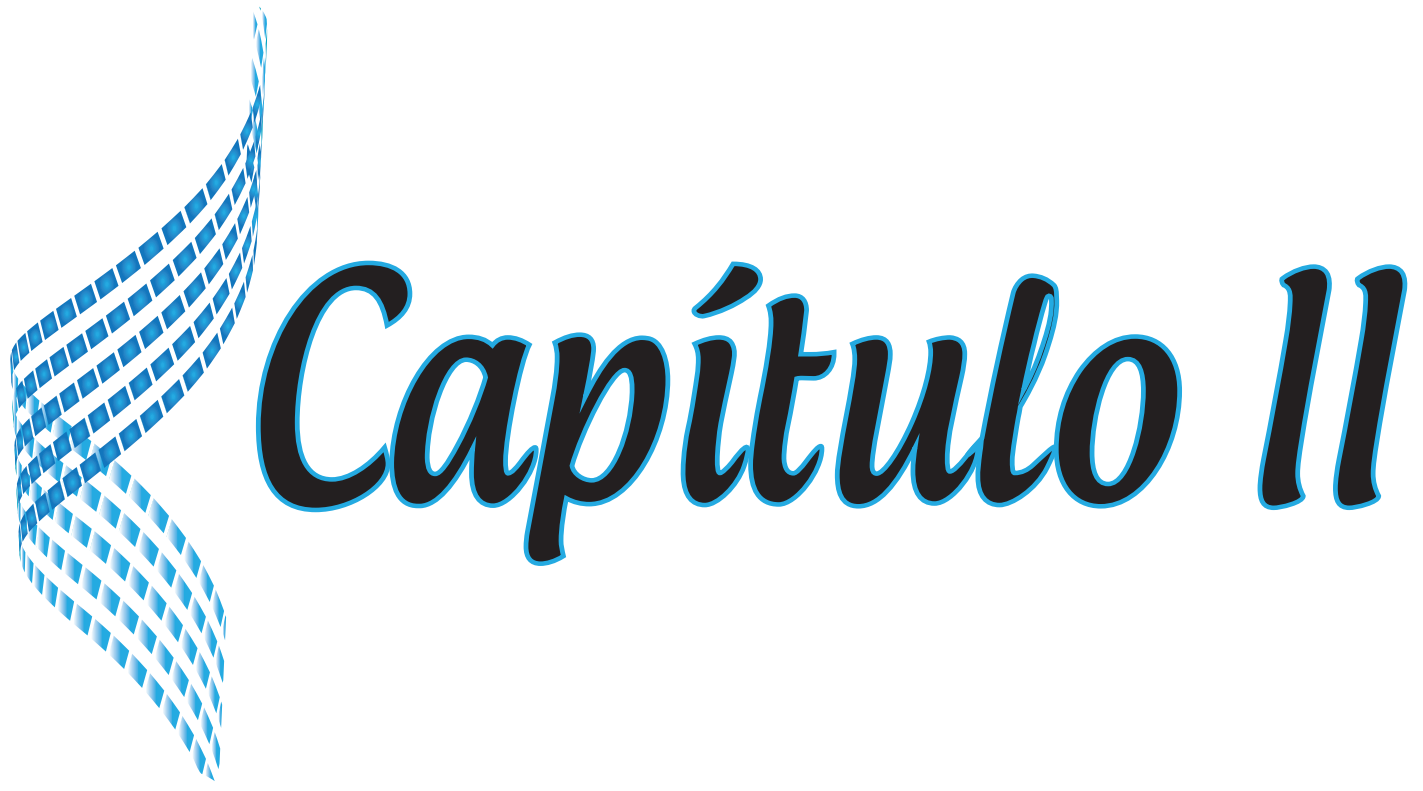

Lo maravilloso de aprender algo, es que nadíe puede arrebatárnoslo.

B.B. King 


\section{Capítulo II}

\section{CAPÍTULO II. Síntesis de ionóforos}

\subsection{INTRODUCCIÓN}

Un sensor químico consta de una parte instrumental (transductor) y de un elemento de reconocimiento denominado receptor, en particular para los electrodos selectivos de iones (ESI) reciben el nombre de ionóforos. En gran medida, estos ionóforos son los responsables de la selectividad en los ESI, ya que este compuesto interaccionará con el analito para el cual fue construido el sensor. La afinidad que tenga el ionóforo hacia este analito determinará que tan selectivo es un sensor frente a posibles interferentes contenidos en la muestra analizada. En este 
capítulo, se expondrá la metodología de síntesis de los compuestos utilizados como ionóforos, los cinco ionóforos expuestos en esta investigación son del tipo ditiofosforados, es decir, tienen un grupo ditiofosfato $\left(\mathrm{PS}_{2}\right)$ en su estructura, por lo tanto de este concepto partiremos.

Los ésteres del ácido ditiofosfórico contienen en su estructura al fragmento $\mathrm{PS}_{2} \mathrm{y}$ al menos un fragmento orgánico. La química de este tipo de compuestos es muy importante debido a que su utilidad se ha extendido a campos como la industria y la medicina. Compuestos de tipo O,O'dialquil(aril)ésteres de ácidos ditiofosfóricos $(\mathrm{RO})_{2} \mathrm{P}(\mathrm{S}) \mathrm{SH}$ son utilizados como inhibidores en la hidrólisis de la enzima acetilcolinesterasa al sustrato acetilcolina la cual es causante de parálisis muscular ${ }^{66}$. De igual manera, los ditiofosfatos han sido utilizados en la industria en el proceso de vulcanización del caucho, como insecticidas, rodenticidas y funguicidas ${ }^{67,68}$ pero actualmente han atraído particularmente la atención por sus propiedades tribológicas ${ }^{69,}{ }^{70}$, propiedades extractantes ${ }^{71.72}$ y como acarreadores en membranas "composite" activadas, debido a la gran afinidad que los átomos de azufre tienen sobre metales blandos del grupo principal y de transición ${ }^{73}, 74,75$. En este sentido la importancia de sintetizar nuevos ligantes azufrados se ha incrementado debido a que presentan propiedades de extracción selectiva a iones metálicos, lo cual es de gran relevancia para su utilidad en la industria minera y en la preservación del medio ambiente ${ }^{76,77,78}$.

La versatilidad de enlace que presenta el fragmento $\mathrm{PS}_{2}{ }^{-}$en los ligantes ditiofosforados da origen a tres modos de coordinación hacia un centro metálico, teniendo la coordinación monodentada I, bidentada asimétrica II y bidentada simétrica III (figura 2.1). 


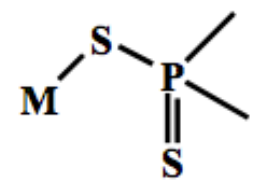

I

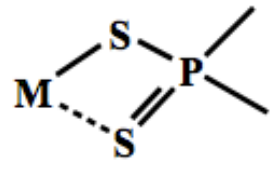

II

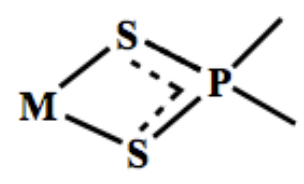

III

Figura 2.1 Modos de coordinación de los compuestos ditiofosforados.

En particular los ionóforos del tipo ditiofosfato, que se sintetizaron en esta investigación son preparados conforme lo reportado por Pérez-García et. al., utilizando la reacción de alcoholes (o fenoles) con decasulfuro de tetrafósforo ${ }^{79}$. Por otra parte, se sabe que los compuestos azufrados presentan afinidad a metales de transición y tomando en cuenta la reactividad que los ionóforos de tipo ditiofosfato presentan hacia el ion $\mathrm{Hg}(\mathrm{II})$, se eligieron compuestos de este tipo para la construcción de electrodos selectivos al ion mercurio. Los cinco compuestos sintetizados son: $\mathrm{O}, \mathrm{O}^{\prime}$-(2,2'-bifenilen)ditiofosfato de metilo, $\mathrm{O}, \mathrm{O}^{\prime}$-(2,2'-bifenilen)ditiofosfato de etilo, $\mathrm{O}, \mathrm{O}^{\prime}$-( $\left(2,2^{\prime}\right.$ bifenilen)ditiofosfato de propilo, O, $\mathrm{O}^{\prime}$-(2,2'-bifenilen)ditiofosfato de butilo y $\mathrm{O}, \mathrm{O}^{\prime}-\left(2,2^{\prime}-\right.$ bifenilen)ditiofosfato de pentilo, por simplificación se hace referencia a ellos como MetDTF, EtDTF, PropDTF, ButDTF y PenDTF respectivamente. 


\subsection{PARTE EXPERIMENTAL}

\subsubsection{MATERIAL Y EQUIPO}

Todas las reacciones fueron realizadas en condiciones anhidras. Los disolventes utilizados fueron secados con sodio metálico. Los compuestos fueron caracterizados por $\mathrm{RMN}$ de ${ }^{1} \mathrm{H}$ en un espectrofotómetro Bruker DMX500 de $500 \mathrm{MHz}$ a $25^{\circ} \mathrm{C}$ en $\mathrm{CDCl}_{3}$.

\subsubsection{SÍNTESIS DEL COMPUESTO FLECHA}

A una solución de bifenol en tolueno se agregó una cantidad igual de decasulfuro de tetrafósforo en moles y la mezcla se colocó a reflojo por 24 horas, después se agregó la parte equimolar de carbonato de potasio y se mantuvo el reflujo por 12 horas. El producto se filtró y se pasó por una columna de sulfato de sodio anhídro; el compuesto precipitó por evaporación lenta del disolvente obteniéndose cristales de O,O'-(2,2'-bifenilen)ditiofosfato de potasio de color blanco.

\subsubsection{SÍNTESIS DE MetDTF $\left(\mathrm{C}_{6} \mathrm{H}_{4} \mathrm{O}\right)_{2} \mathrm{P}(\mathrm{S}) \mathrm{S}-\mathrm{CH}_{3}$}

A una solución de O,O'-(2,2’'-bifenilen)ditiofosfato de potasio (1.00 g, $3.13 \mathrm{mmol})$ en isopropanol $(20 \mathrm{~mL})$ se agregaron $0.19 \mathrm{~mL}(3.13 \mathrm{mmol})$ de yodometano y la mezcla resultante se refluyó durante 12 h. El producto se filtró y se pasó por una columna de sulfato de sodio anhidro; 
el compuesto precipitó por evaporación lenta del disolvente obteniéndose cristales incoloros. P.f. 57.2-58.5 ${ }^{\circ} \mathrm{C}$, rendimiento $83 \%$, RMN de ${ }^{1} \mathrm{H}\left(\mathrm{CDCl}_{3}\right)$ para la parte alifática: $\delta(\mathrm{ppm}) ; 3.14(\mathrm{dc}$, $\left.2 \mathrm{H},{ }^{3} \mathrm{~J}_{\mathrm{H}-\mathrm{P}}=19.86, \mathrm{CH}_{2}\right), 1.48\left(\mathrm{t}, 3 \mathrm{H}, \mathrm{CH}_{3}\right)$.

\subsubsection{SÍNTESIS DE Et-DTF $\left(\mathrm{C}_{6} \mathrm{H}_{4} \mathrm{O}_{2} \mathrm{P}(\mathrm{S}) \mathrm{S}-\mathrm{CH}_{2} \mathrm{CH}_{3}\right.$}

A una solución de O,O'-(2,2'-bifenilen)ditiofosfato de potasio (1.00 g, $3.13 \mathrm{mmol})$ en isopropanol $(20 \mathrm{~mL})$ se agregaron $0.25 \mathrm{~mL}(3.13 \mathrm{mmol})$ de Bromoetano y la mezcla resultante se refluyó durante 12 h. El producto se filtró y se pasó por una columna de sulfato de sodio anhidro; el compuesto precipitó por evaporación lenta del disolvente obteniéndose cristales incoloros. P.f. 86-88 ${ }^{\circ} \mathrm{C}$, rendimiento $95 \%, \mathrm{RMN}$ de ${ }^{1} \mathrm{H}\left(\mathrm{CDCl}_{3}\right)$ para la parte alifática: $\delta(\mathrm{ppm}) ; 2.50(\mathrm{~d}, 3 \mathrm{H}$, $\left.{ }^{3} \mathrm{~J}_{\mathrm{H}-\mathrm{P}}=18.88, \mathrm{CH}_{3}\right)$.

\subsubsection{SÍNTESIS DE Prop-DTF $\left(\mathrm{C}_{6} \mathrm{H}_{4} \mathrm{O}\right)_{2} \mathrm{P}(\mathrm{S}) \mathrm{S}-\left(\mathrm{CH}_{2}\right)_{2} \mathrm{CH}_{3}$}

A una solución de $\mathrm{O}, \mathrm{O}^{\prime}$-(2,2'-bifenilen)ditiofosfato de potasio (1.00 g, $\left.3.13 \mathrm{mmol}\right)$ en isopropanol $(20 \mathrm{~mL})$ se agregaron $0.29 \mathrm{~mL}(3.13 \mathrm{mmol})$ de $\mathrm{n}$-Bromopropano y la mezcla resultante se refluyó durante 12 h. El producto se filtró y se pasó por una columna de sulfato de sodio anhidro; el compuesto precipitó por evaporación lenta del disolvente obteniéndose cristales incoloros. P.f. $55-56{ }^{\circ} \mathrm{C}$, rendimiento $89 \%$. RMN de ${ }^{1} \mathrm{H}\left(\mathrm{CDCl}_{3}\right)$ para la parte alifática: $\delta(\mathrm{ppm})$; $3.11\left(\mathrm{dt}, 2 \mathrm{H},{ }^{3} \mathrm{~J}_{\mathrm{H}-\mathrm{P}}=19.44, \mathrm{CH}_{2}\right), 1.84$ (séxtuple, $\left.2 \mathrm{H}, \mathrm{CH}_{2}\right), 1.06\left(\mathrm{t}, 3 \mathrm{H}, \mathrm{CH}_{3}\right)$. 


\subsubsection{SÍNTESIS DE But-DTF $\left(\mathrm{C}_{6} \mathrm{H}_{4} \mathrm{O}\right)_{2} \mathrm{P}(\mathrm{S}) \mathrm{S}-\left(\mathrm{CH}_{2}\right)_{3} \mathrm{CH}_{3}$}

A una solución de O,O'-(2,2'-bifenilen)ditiofosfato de potasio (1.00 g, $3.13 \mathrm{mmol})$ en isopropanol $(20 \mathrm{~mL})$ se agregaron $0.34 \mathrm{~mL}(3.13 \mathrm{mmol})$ de $\mathrm{n}$-Bromobutano y la mezcla resultante se refluyó durante $12 \mathrm{~h}$. El producto se filtró y se pasó por una columna de sulfato de sodio anhidro; el compuesto precipitó por evaporación lenta del disolvente obteniéndose un líquido color amarillo, rendimiento $81 \%$. $\mathrm{RMN}$ de ${ }^{1} \mathrm{H}\left(\mathrm{CDCl}_{3}\right)$ para la parte alifática: $\delta(\mathrm{ppm})$; $3.12\left(\mathrm{dt}, 2 \mathrm{H},{ }^{3} \mathrm{~J}_{\mathrm{H}-\mathrm{P}}=19.20, \mathrm{CH}_{2}\right.$ ), 1.78 (quíntuple, $2 \mathrm{H}, \mathrm{CH}_{2}$ ), 1.46 (séxtuple, $2 \mathrm{H}, \mathrm{CH}_{2}$ ), 0.95 (t, 3H, $\left.\mathrm{CH}_{3}\right)$.

\subsubsection{SÍNTESIS DE Pen-DTF $\left(\mathrm{C}_{6} \mathrm{H}_{4} \mathrm{O}\right)_{2} \mathrm{P}(\mathrm{S}) \mathrm{S}-\left(\mathrm{CH}_{2}\right)_{4} \mathrm{CH}_{3}$}

A una solución de O,O'-(2,2'-bifenilen)ditiofosfato de potasio (1.00 g, $3.13 \mathrm{mmol})$ en isopropanol $(20 \mathrm{~mL})$ se agregaron $0.39 \mathrm{~mL}(3.13 \mathrm{mmol})$ de $\mathrm{n}$-Bromopentano y la mezcla resultante se refluyó durante 12 h. El producto se filtró y se pasó por una columna de sulfato de sodio anhidro; el compuesto precipitó por evaporación lenta del disolvente obteniéndose un líquido color amarillo, rendimiento $78 \%$. RMN de ${ }^{1} \mathrm{H}\left(\mathrm{CDCl}_{3}\right)$ para la parte alifática: $\delta(\mathrm{ppm})$; $3.10\left(\mathrm{dt}, 2 \mathrm{H},{ }^{3} \mathrm{~J}_{\mathrm{H}-\mathrm{P}}=19.25 \mathrm{~Hz}\right), 1.78$ (quinteto, $\left.2 \mathrm{H}, \mathrm{CH}_{2}{ }^{\mathrm{a}}\right), 1.38\left(\mathrm{~m}, 2 \mathrm{H}, \mathrm{CH}_{2}{ }^{\mathrm{b}}\right), 1.38\left(\mathrm{~m}, 2 \mathrm{H}, \mathrm{CH}_{2}^{\mathrm{c}}\right.$ ), $0.89\left(\mathrm{t}, 3 \mathrm{H}, \mathrm{CH}_{3}\right)$. 


\subsection{RESULTADOS Y DISCUSIÓN}

\subsubsection{SÍNTESIS DE LOS IONÓFOROS}

Para la síntesis de los cinco ionóforos, se hizo reaccionar bifenol con decasulfuro de tetrafósforo para la obtención del compuesto flecha, después el compuesto flecha reaccionó con el correspondiente halogenuro de alquilo, donde el grupo alquilo va de radical metilo a pentilo para la obtención de los correspondientes ésteres ditiofosforados (Figura 2.2).
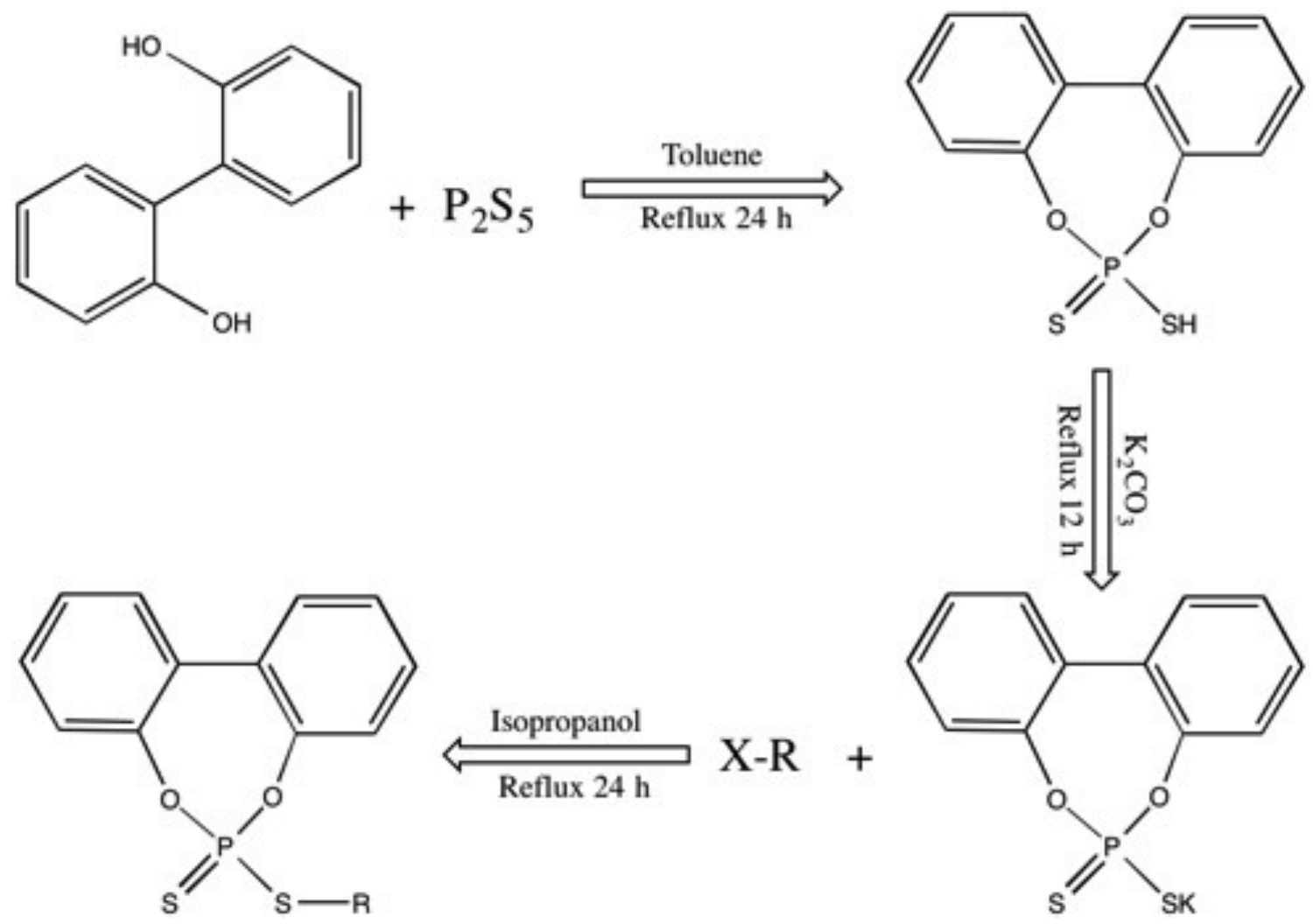

Figura 2.2. Ruta de síntesis para los ionóforos MetDTF, EtDTF, PropDTF, ButDTF y PenDTF ("R” en la estructura representa a la cadena alquílica). 
Todos los compuestos son estables al medio ambiente y solubles en cloroformo y tolueno e insolubles en agua, con lo que se cumple con la hidrofobicidad requerida para un compuesto acarreador. Algunas de las propiedades físicas y químicas de los compuestos se encuentran enlistadas en la Tabla 2.1.

Tabla 2.1. Propiedades físicas de los ionóforos.

\begin{tabular}{ccc}
\hline Ionóforo & Estado físico & Punto de fusión ${ }^{\circ} \mathbf{C}$ \\
\hline MetDTF & Sólido blanco & 57 \\
PropEDT & Sólido blanco & 86 \\
ButDTF & Sólido blanco & 55 \\
PenDTF & Líquido viscoso café & - \\
\hline
\end{tabular}

Sin duda la presencia de grupos alquilo origina cambios importantes en el resto de la molécula. Los compuestos metilo, etilo y propilo son sólidos a temperatura ambiente; sin embargo, los compuestos de butilo y pentilo resultaron ser líquidos. La naturaleza de la unión del grupo ditiofosfato hacia el grupo R, deja solamente como posibilidad de enlace con otra especie el enlace de coordinación (por la presencia de pares de electrones libres en los átomos de azufre) dicho enlace suele ser más débil que un enlace covalente puro. 


\subsubsection{ESPECTROSCOPÍA DE RESONANCIA MAGNÉTICA NUCLEAR}

Los espectros de $\mathrm{RMN}$ de ${ }^{1} \mathrm{H}$ de los compuestos se obtuvieron en $\mathrm{CDCl}_{3}$ a $25{ }^{\circ} \mathrm{C}$. Los espectros de $\mathrm{RMN}$ de ${ }^{1} \mathrm{H}$ para los cinco ionóforos muestran cuatro señales para un sistema orto sustituido en la zona de aromáticos; mientras que en la región alifática la señal más relevante corresponde a los protones unidos al carbono alfa a azufre la cual se muestra como una señal con multiplicidad doble para el ionóforo MetDTF, doble de cuádruple para EtDTF y doble de triple para los restantes (PropDTF, ButDTF y PenDTF); dicha multiplicidad se deriva del acoplamiento a tres enlaces con el átomo de fósforo con valores aproximados de ${ }^{3} J_{H-P}=19 \mathrm{~Hz}$ para los cinco compuestos. En la figura 2.3 se muestran los espectros de resonancia magnética nuclear $(\mathrm{RMN})$ de ${ }^{1} \mathrm{H}$ en $\mathrm{CDCl}_{3}$ a $25{ }^{\circ} \mathrm{C}$. Los desplazamientos químicos para la parte alifática son: $\delta(\mathrm{ppm})$ : EtDTF; $2.50\left(\mathrm{~d}, 3 \mathrm{H},{ }^{3} \mathrm{~J}_{\mathrm{H}-\mathrm{P}}=18.88, \mathrm{CH}_{3}\right)$ : MetDTF; $3.14\left(\mathrm{dc}, 2 \mathrm{H},{ }^{3} \mathrm{~J}_{\mathrm{H}-\mathrm{P}}=19.86, \mathrm{CH}_{2}\right)$, $1.48\left(\mathrm{t}, 3 \mathrm{H}, \mathrm{CH}_{3}\right)$ : PropDTF; $3.11\left(\mathrm{dt}, 2 \mathrm{H},{ }^{3} \mathrm{~J}_{\mathrm{H}-\mathrm{P}}=19.44, \mathrm{CH}_{2}\right), 1.84$ (séxtuple, $\left.2 \mathrm{H}, \mathrm{CH}_{2}\right), 1.06(\mathrm{t}$, $3 \mathrm{H}, \mathrm{CH}_{3}$ ) y ButDTF; $3.12\left(\mathrm{dt}, 2 \mathrm{H},{ }^{3} \mathrm{~J}_{\mathrm{H}-\mathrm{P}}=19.20, \mathrm{CH}_{2}\right.$ ), 1.78 (quíntuple, $2 \mathrm{H}, \mathrm{CH}_{2}$ ), 1.46 (séxtuple, $\left.2 \mathrm{H}, \mathrm{CH}_{2}\right), 0.95\left(\mathrm{t}, 3 \mathrm{H}, \mathrm{CH}_{3}\right)$. 

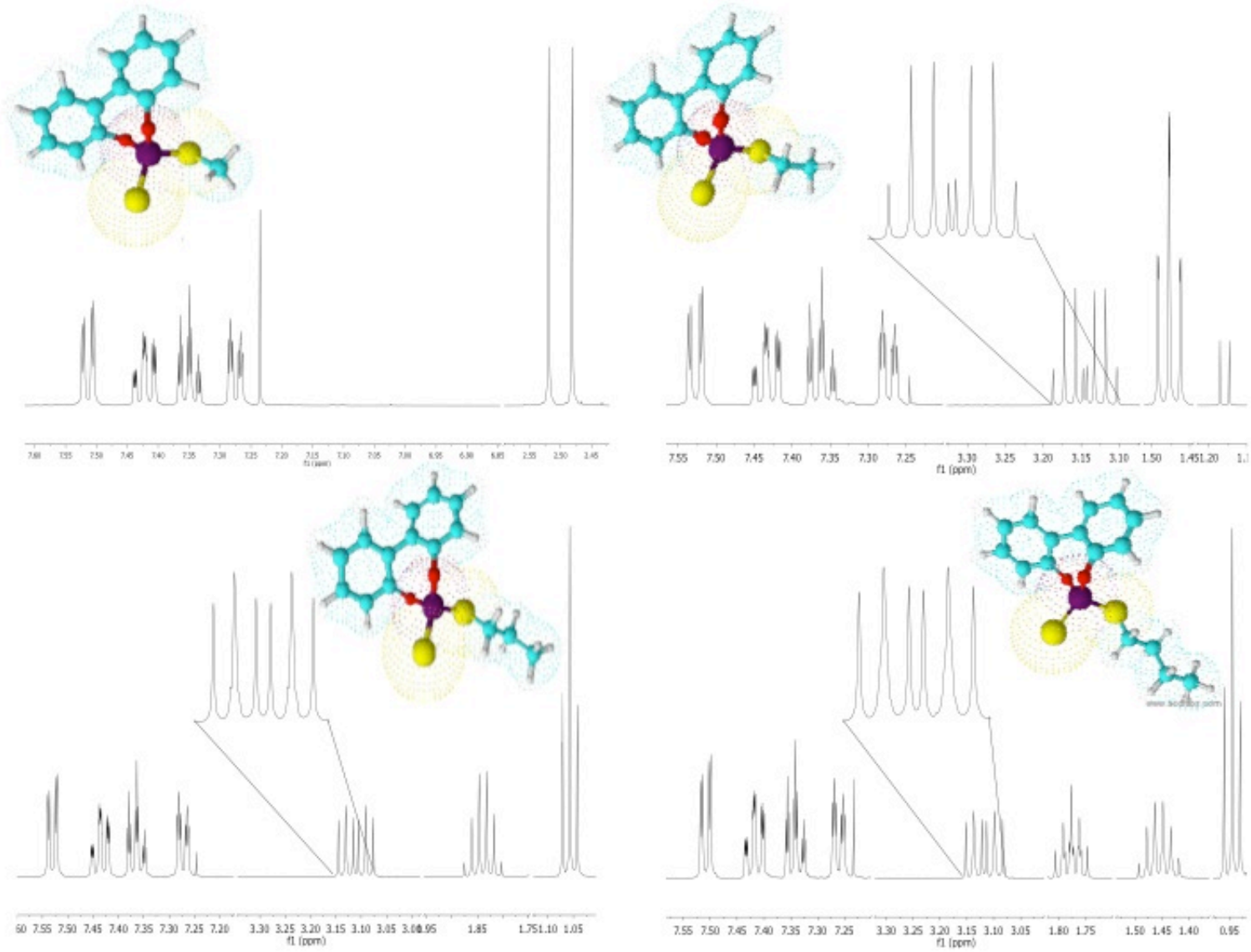

Figura 2.3. Espectros de $\mathrm{RMN}$ de ${ }^{1} \mathrm{H}$ en $\mathrm{CDCl}_{3}$ para MetDTF, EtDTF, PropDTF y ButDTF.

\subsection{CONCLUSIONES}

La síntesis para la obtención de ionóforos a partir del compuesto flecha con el incremento de la cadena alquílica, dio como resultado que de los cinco compuestos preparados, los compuestos ButDTF y PenDTF que contienen el grupo n-butilo y n-pentilo respectivamente fueron líquidos a temperatura ambiente, los restantes ionóforos fueron sólidos. Todos los ionóforos fueron caracterizados por $\mathrm{RMN}$ de ${ }^{1} \mathrm{H}$ en cloroformo deuterado, dando como resultado las bandas 
características de los grupos alquílicos sustituyentes garantizando la estructura deseada. Los ionóforos podrán ser utilizados para la construcción de sensores para la determinación de $\mathrm{Hg}(\mathrm{II})$. 


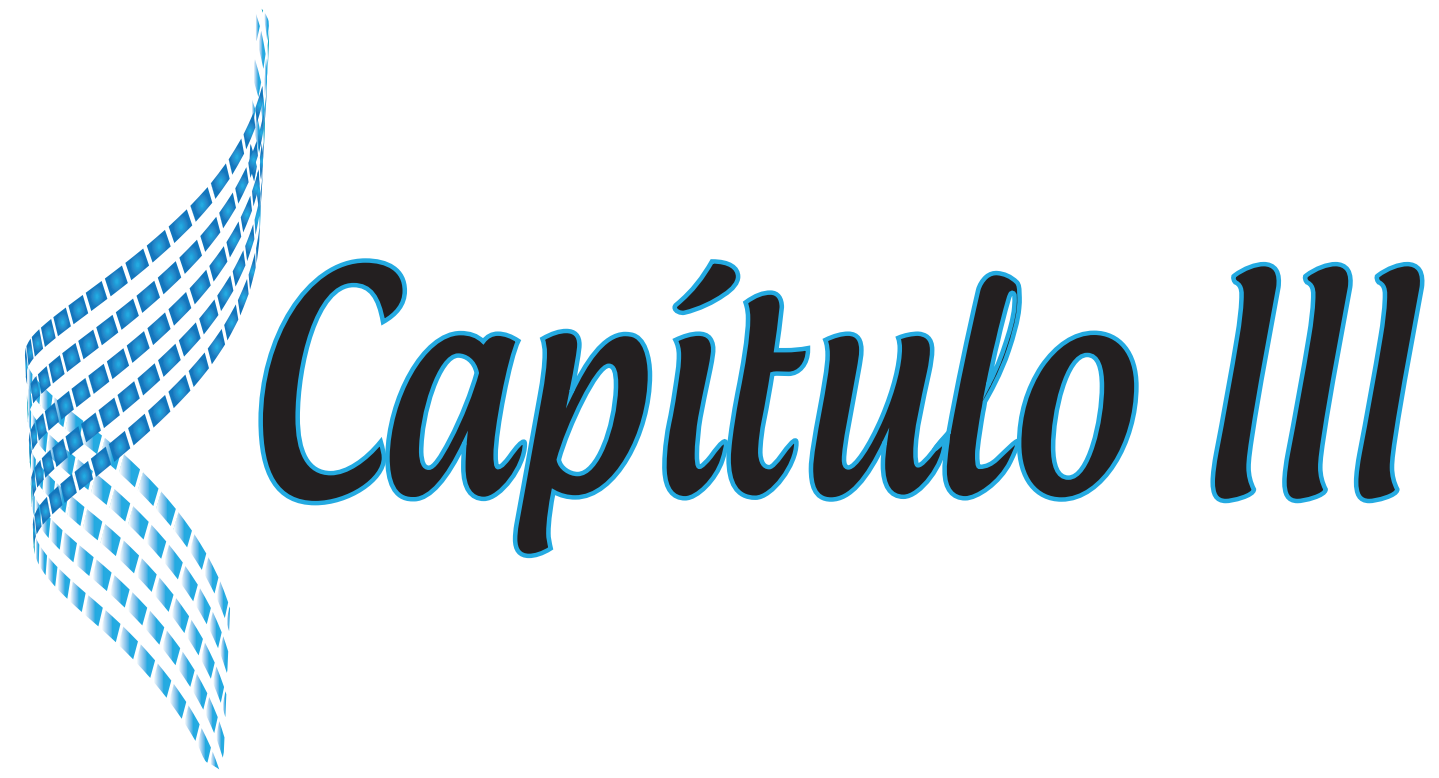

Educación es to que queda después de ofvidar lo que se ha aprendido en la escuela. Albert Finstein 


\section{Capítulo III}

\section{CAPÍTULO III. ELECTRODO POTENCIOMÉTRICO DE CONTACTO INTERNO SÓLIDO Y DE SUPERFICIE RENOVABLE BASADO EN UN NUEVO IONÓFORO DITIOFOSFORADO SELECTIVO A Hg(II)}

\subsection{INTRODUCCIÓN}

El mercurio es generalmente encontrado en concentraciones muy bajas en el medio ambiente y puede ser absorbido fácilmente por el ser humano y otros organismos vivos ${ }^{80,81}$. La bioacumulación de mercurio en el ser humano es altamente tóxica para el riñón y puede causar daños neurológicos, parálisis y hasta problemas en el parto. El mercurio puede encontrase en diferentes formas químicas; pero tanto la forma elemental como la inorgánica puede ser transformada por sistemas biológicos en la forma orgánica en compuestos alquílicos de cadenas 
cortas, los cuales es sabido que son responsables de daños al sistema nervioso central ${ }^{82}$. Desde la edad media, las sales de mercurio han sido ampliamente utilizadas para varias actividades humanas; en la medicina por ejemplo, se han utilizado como ungüentos para la piel y ojos. En tiempos recientes compuestos de mercurio son utilizados en diuréticos, amalgamas dentales, antisépticos etc. ${ }^{83,84,85}$.

Debido al gran impacto que este ión tiene en el medio ambiente y en la salud humana, existe una gran necesidad para desarrollar nuevos métodos químicos para la determinación de mercurio en bajas concentraciones ${ }^{86,87}$.

Los método clásicos que se han utilizado en la determinación de mercurio y que son caracterizados por tener un bajo límite de detección son técnicas como la espectroscopia de absorción atómica (AAS), la espectrometría de masas con plasma de acoplamiento inductivo (ICP-MS), la electroforesis capilar, entre otras, pero son técnicas de alto costo que no pueden ser utilizadas en análisis de campo ${ }^{88,89}$.

Entre las diferentes técnicas para el análisis de metales pesados, el análisis electroquímico es considerado una de las más poderosas técnicas dentro de este campo; sin embargo, el uso de celdas electroquímicas convencionales y electrodos voluminosos, presentan un problema para las mediciones, debido a la gran cantidad de volumen de la solución muestra que se requiere ${ }^{90}$.

Los sensores potenciométricos ofrecen muchas ventajas como son: bajo costo, de fácil fabricación, bajos límites de detección, amplio rango de respuesta, una alta selectividad, entre $\operatorname{otras}^{91,92}$.

Diversos reportes sobre electrodos selectivos de iones (ESI) que utilizan ligantes neutros para la detección de $\mathrm{Hg}(\mathrm{II})$, son encontrados en la literatura, en ellos es posible ver que la configuración de la superficie de contacto es variada ${ }^{93,94,95,96}$ ya que en algunos casos el ligante 
es encapsulado en una membrana polimérica con el fin de disminuir la pérdida de éste en el electrodo; sin embargo, se ha encontrado que la superficie sufre daños irreversibles conforme se agrega $\mathrm{Hg}(\mathrm{II})$ a la solución, lo que produce respuesta erróneas después de cada experimento [50].

Diferentes ionóforos han sido sintetizados y utilizados para la detección selectiva de $\operatorname{Hg}($ II), ligantes como tioles ${ }^{97}$, bases de Schiff ${ }^{98}$, tioureas ${ }^{99}$, y derivados de calixarenos ${ }^{100}$ entre otros han sido utilizados bajo condiciones donde el ion nitrato $\left(\mathrm{NO}_{3}{ }^{-}\right)$fue la especie aniónica predomínate, esto debido a la baja solubilidad de especies de mercurio con contraiones diferentes al nitrato.

Es sabido que el $\mathrm{Hg}(\mathrm{II})$ muestra gran afinidad a ligantes que contienen átomos de azufre y por lo tanto han sido utilizados con éxito como ionóforos para este metal, dicha afinidad ha sido comprobada utilizando cálculos de la teoría de funcionales de la densidad ${ }^{101,102}$.

En este capítulo se presentan los estudios potenciométricos para la detección de $\mathrm{Hg}(\mathrm{II})$ de un nuevo electrodo selectivo de iones de contacto interno sólido, el composite se construyó haciendo una mezcla 1:1 de resina epóxica/grafito, luego ésta se mezcla con el ionóforo PenDTF, una vez hecho el electrodo puede ser utilizado para varios experimentos renovando la superficie de contacto con un simple tratamiento con papel abrasivo.

El compuesto químico utilizado como ionóforo (PenDTF figura 3.1) es del tipo ditiofosforado, el cual en estudios de extracción y transporte, mostró una alta selectividad a $\mathrm{Hg}$ (II) frente a diferentes iones metálicos ${ }^{103}$. Químicamente el ligante PenDTF es un compuesto neutro pero con átomos donadores como el azufre que tienen pares de electrones libres que pueden coordinar a los iones metálicos; además este ligante es hidrofóbico lo que favorece los estudios potenciométricos en medio acuoso. 


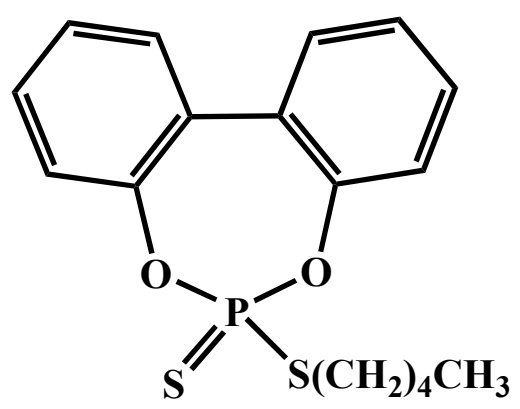

Figura 3.1. Estructura del ligante $O, \mathrm{O}^{\prime}$-(2,2'-bifenilen)ditiofosfato de pentilo (PenDTF).

\subsection{EXPERIMENTAL}

\subsubsection{DISEÑO DEL ELECTRODO Y OPTIMIZACIÓN}

En un recipiente de vidrio se agregan Araldita y endurecedor HY (Ciba-Geigy), grafito y el ligante PenDTF conforme a la Tabla 3.1, se mezclan estos componentes hasta obtener una pasta homogénea y parte de esta mezcla se deposita en tubo de PVC de $7 \mathrm{~mm}$ de diámetro aproximadamente, el cual tiene dentro un contacto sólido de cobre que sirve como soporte de la mezcla. El dispositivo se deja curar por 24 horas. a $40{ }^{\circ} \mathrm{C}$ y se acondiciona en solución de $1.0 \times 10^{-3} \mathrm{~mol} \mathrm{~L}^{-1}$ de $\mathrm{Hg}\left(\mathrm{NO}_{3}\right)_{2}$ (Sigma-Aldrich) durante cinco minutos antes de cada prueba. En la figura 3.2 se muestra un esquema de la construcción del electrodo.

El análisis de $\mathrm{Hg}(\mathrm{II})$ se realizó mediante cronopotenciometría a corriente nula utilizando un potenciostato Epsilon BASi EW-8111. Se utilizó un sistema de dos electrodos donde el electrodo de trabajo fue el obtenido con el ligante PenDTF y $\mathrm{Ag} / \mathrm{AgCl}$ (Metrohm 6.0736.110) como referencia. Todos los experimentos se realizaron en una celda electroquímica convencional de 
vidrio con capacidad de $20 \mathrm{~mL}$ y se utilizaron $10 \mathrm{~mL}$ de una solución de $\mathrm{HClO}_{4}$ (J.T BAKER) $1.0 \mathrm{~mol} \mathrm{~L}^{-1}$. Se prepararon soluciones de $\mathrm{Hg}(\mathrm{II})$ por dilución hasta $1.0 \times 10^{-6} \mathrm{~mol} \mathrm{~L}^{-1}$ y se agregaron alicuotas de ellas para el aumento en la concentracion total de la celda. Se mide el potencial resultante para cada concentracion y se grafica contra el logaritmo decimal de la concentración. Cada electrodo se prueba por triplicado lijando la superficie con papel abrasivo antes de cada prueba.

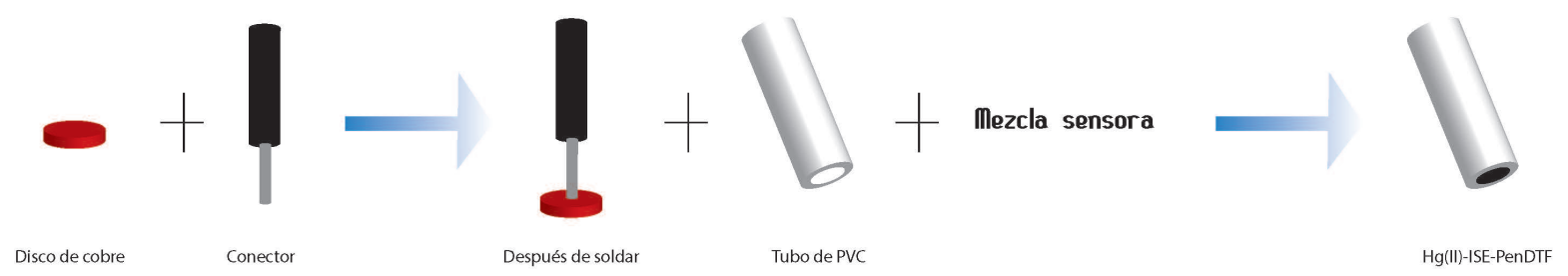

Figura 3.2. Esquema de construcción de los sensores.

\subsubsection{EFECTO DEL $\mathrm{pH}$}

Seleccionada la óptima composición que resulta ser la del electrodo Hg(II)-ISE-PenDTF 1, se estudia la la influencia del $\mathrm{pH}$ sobre la respuesta potenciométrica del electrodo, y esto se hace variando el $\mathrm{pH}$ en un intervalo de 0-7 con solución de $\mathrm{HNO}_{3}$ (J.T BAKER) 1,0 mol L-1 y adicionando $\mathrm{KOH}$ (J.T BAKER) $1,0 \mathrm{~mol} \mathrm{~L}^{-1}$ en concentración constante de $\mathrm{Hg}\left(\mathrm{NO}_{3}\right)_{2} 1,0 \times 10^{-3}$ mol L $\mathrm{L}^{-1}$. Para cada $\mathrm{pH}$ se mide el potencial del electrodo a la misma concentración de iones $\operatorname{Hg}(\mathrm{II})$. 


\subsection{RESULTADOS Y DISCUSIÓN}

\subsubsection{OPTIMIZACIÓN DE LA COMPOSICIÓN DEL ELECTRODO}

En la composición de los electrodos selectivos de iones (ESI) una parte primordial es su elemento de reconocimiento, así como la naturaleza de los aditivos utilizados en su construcción ya que de estos dependen en gran medida todos sus parámetros importantes como la sensibilidad y la selectividad. Así, diferentes aspectos en la composición se optimizaron, la influencia de la composición del "composite" sobre la respuesta potenciométrica del electrodo, fué analizada mediante un diseño factorial del tipo $2^{3}$ (ver anexo B), los tres factores analizados fueron la resina, el endurecedor y la concentración del ionóforo, el nivel alto de cada factor fue 250, 100 y $20 \mathrm{mg}$ respectivamente, así como el nivel bajo de cada factor fue 200, 50 y $10 \mathrm{mg}$, y dejando la cantidad de grafito en el composite constante. En la Tabla 3.1 se muestra la composición de los diferentes electrodos construidos con sus parámetros analíticos. La respuesta potenciométrica de los sensores se probó en un amplio intervalo de concentraciones de $\operatorname{Hg}(\mathrm{II})\left(1,0 \times 10^{-9}\right.$ a 1,0 x $10^{-2}$ mol L'-1) en solución $1.0 \mathrm{~mol} \mathrm{~L}^{-1}$ de $\mathrm{HClO}_{4}$. 
Tabla 3.1. Diseño factorial aplicado a la composición del electrodo.

\begin{tabular}{|c|c|c|c|c|c|c|c|}
\hline \multirow[t]{2}{*}{ Hg(II)-ISE-PenDTF* } & \multicolumn{4}{|c|}{ Composición del electrodo (mg) } & \multirow[t]{2}{*}{ Pendiente $\left(\mathrm{mV}\right.$ década $\left.^{-1}\right)$} & \multirow[t]{2}{*}{ LDD $\left(\mathrm{mol} \mathrm{L}^{-1}\right)$} & \multirow[t]{2}{*}{ Intervalo lineal $\left(\mathrm{mol} \mathrm{L}^{-1}\right)$} \\
\hline & Grafito & Resina & Endurecedor & Ionóforo & & & \\
\hline $\mathbf{1}$ & 350 & 250 & 100 & 20 & $33.81 \pm 1.24$ & $4.24 \times 10^{-7} \pm 2.48 \times 10^{-7}$ & $5.35 \times 10^{-7}-1.00 \times 10^{-2}$ \\
\hline 2 & 350 & 250 & 50 & 20 & $145.61 \pm 27.38$ & $2.36 \times 10^{-5} \pm 5.48 \times 10^{-6}$ & $4.99 \times 10^{-5}-1.00 \times 10^{-2}$ \\
\hline 3 & 350 & 200 & 50 & 20 & $100.02 \pm 11.59$ & $6.68 \times 10^{-5} \pm 1.10 \times 10^{-5}$ & $9.24 \times 10^{-5}-1.00 \times 10^{-2}$ \\
\hline 4 & 350 & 200 & 50 & 10 & $70.95 \pm 7.99$ & $2.29 \times 10^{-5} \pm 3.54 \times 10^{-6}$ & $4.99 \times 10^{-5}-1.00 \times 10^{-2}$ \\
\hline 5 & 350 & 200 & 100 & 10 & $32.05 \pm 0.77$ & $1.94 \times 10^{-6} \pm 8.55 \times 10^{-7}$ & $3.64 \times 10^{-6}-1.00 \times 10^{-2}$ \\
\hline 6 & 350 & 250 & 100 & 10 & $37.98 \pm 2.51$ & $1.58 \times 10^{-4} \pm 4.14 \times 10^{-5}$ & $1.76 \times 10^{-4}-1.00 \times 10^{-2}$ \\
\hline 7 & 350 & 200 & 100 & 20 & $21.4 \pm 1.65$ & $1.05 \times 10^{-5} \pm 8.22 \times 10^{-7}$ & $1.56 \times 10^{-5}-1.00 \times 10^{-2}$ \\
\hline 8 & 350 & 250 & 50 & 10 & $94.54 \pm 7.08$ & $1.96 \times 10^{-5} \pm 6.50 \times 10^{-6}$ & $4.99 \times 10^{-5}-1.00 \times 10^{-2}$ \\
\hline
\end{tabular}

El diseño factorial muestra que en los niveles bajos de endurecedor se obtienen pendientes muy por encima de la esperada según la ecuación de Nernst y límites de detección alrededor de $10^{-5}$ mol L $\mathrm{L}^{-1}$. A partir de los datos resumidos de la Tabla 3.1 los distintos electrodos para $\mathrm{Hg}$ (II) basados en PenDTF, se revela que el electrodo 1 es el sensor que expone las mejores características analíticas, el mejor comportamiento Nernstiano, la mejor sensibilidad y un amplio rango de linealidad (Figura 3.4), por lo que se eligió como electrodo de trabajo para los experimentos posteriores. 


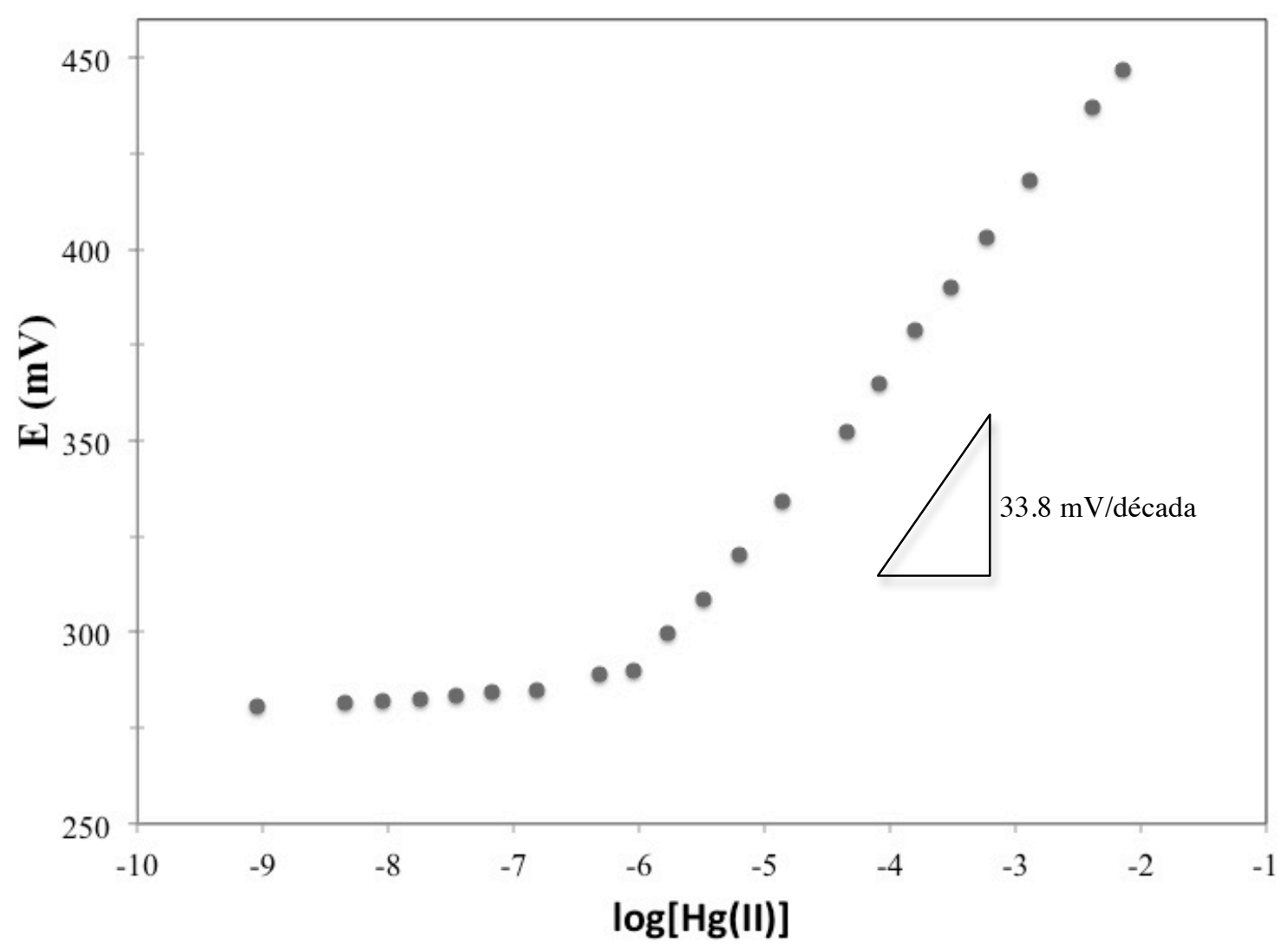

Figura 3.4. Curva de calibración del electrodo selectivo a $\mathrm{Hg}(\mathrm{II})$ basado en PenDTF.

\subsubsection{REPRODUCIBILIDAD Y EFECTO DE LIJAR LA SUPERFICIE DEL ELECTRODO}

Para comprobar la reproducibilidad del electrodo $\mathrm{Hg}(\mathrm{II})$-ISE-PenDTF 1 se construyeron electrodos de la misma composición en diferentes momentos. En la Tabla 3.2 se muestran los resultados para cinco electrodos diferentes pero con la misma composición. Se observa en los parámetros analíticos como la pendiente y límite de detección que la variación no es significativa (menor al 3\%). Por lo tanto, puede garantizar la reproducibilidad de electrodo construido. 
Tabla 3.2. Respuesta de los diferentes electrodos construidos en diferentes momentos utilizando la composición Hg-(II)-ISE-PenDTF 1.

\begin{tabular}{cclc}
\hline Hg(II)-ISE-PenDTF 1 & $\begin{array}{c}\text { Pendiente } \\
(\mathrm{mV} / \text { década })\end{array}$ & & \\
\hline $\mathrm{a}$ & $32.6 \pm 1.3$ & $(6.9 \pm 3.5) \times 10^{-7}$ & 0.996 \\
$\mathrm{~b}$ & $35.3 \pm 0.8$ & $(8.0 \pm 5.6) \times 10^{-7}$ & 0.997 \\
$\mathrm{c}$ & $34.5 \pm 1.4$ & $(7.9 \pm 3.4) \times 10^{-7}$ & 0.995 \\
$\mathrm{~d}$ & $32.2 \pm 0.7$ & $(6.8 \pm 1.5) \times 10^{-7}$ & 0.990 \\
$\mathrm{e}$ & $34.6 \pm 1.0$ & $(7.0 \pm 3.6) \times 10^{-7}$ & 0.994 \\
\hline
\end{tabular}

La figura 3.5 muestra las curvas de calibración para el electrodo $\mathrm{Hg}$ (II)-ISE-PenDTF 1, se renovó cinco veces la superficie con papel abrasivo, se puede ver que no hay una diferencia significativa entre curvas ya que conservan su tendencia y las pendientes son estadísticamente muy parecidas; por tanto se puede garantizar la reproducibilidad del sensor después de ser renovado. 


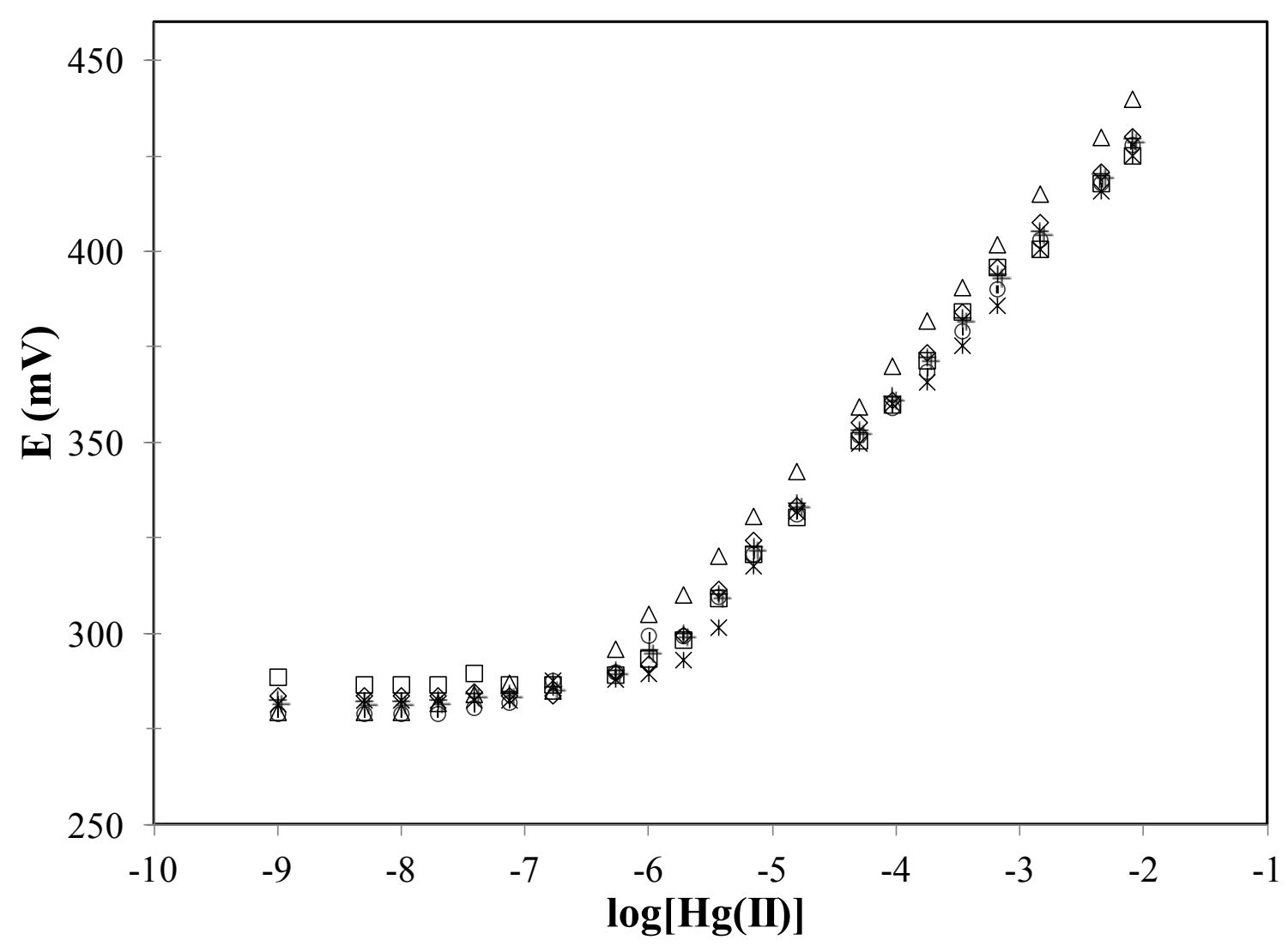

Figura 3.5. Curvas de calibración para el electrodo $\mathrm{Hg}(\mathrm{II})$-ISE-PenDTF 1 renovando la superficie con papel abrasivo.

\subsubsection{RESPUESTA ANIÓNICA}

Los electrodos basados en una matriz carbón-epoxy típicamente presentan respuesta aniónica, lo cual es indeseable para los ESI, así que se examinó la respuesta potenciométrica del electrodo $\mathrm{Hg}$ (II)-ISE-PenDTF 1 para diferentes aniones y con diferentes cargas como: $\mathrm{COO}^{-}, \mathrm{CO}_{3}^{2-}, \mathrm{Cl}^{-} \mathrm{y}$ $\mathrm{SO}_{4}{ }^{2-}$, la figura 3.6 muestra que el electrodo que aquí se propone no muestra ningún cambio en el potencial a diferentes concentraciones de los diferentes aniones y esto muestra que, si bien los 
electrodos de tipo epoxy pueden presentar respuesta aniónica por contener disponibles en su configuración sitios aniónicos, en este caso con la inclusión del ionóforo PenDTF en la matriz epoxy tales sitios quedan bloqueados o al menos se reducen al mínimo.

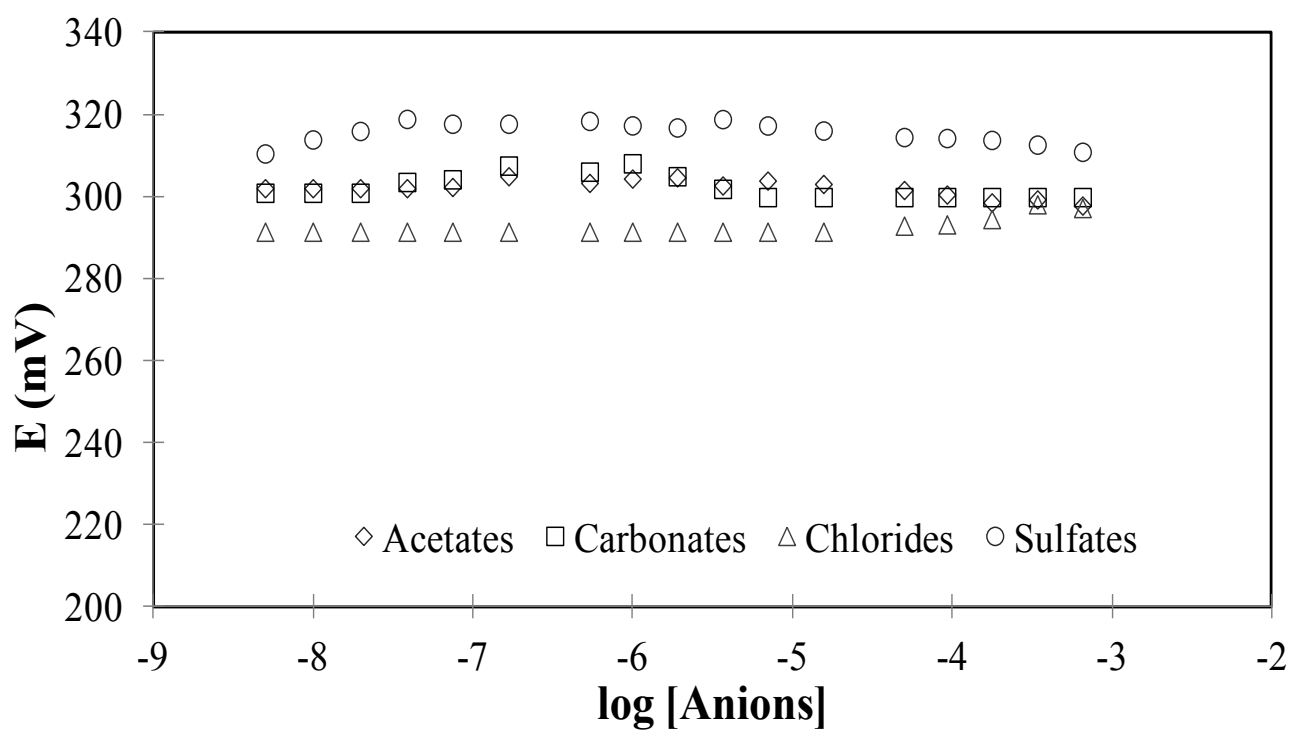

Figura 3.6. Respuesta potenciométrica del electrodo $\mathrm{Hg}(\mathrm{II})$-ISE-PenDTF 1 para diferentes aniones.

\subsubsection{EFECTO DEL PH SOBRE LA RESPUESTA DEL ELECTRODO}

La influencia del pH sobre el ESI se estudió utilizando una solución de $\mathrm{Hg}\left(\mathrm{NO}_{3}\right)_{2} 1,0 \times 10^{-3} \mathrm{~mol}$ $\mathrm{L}^{-1}$, el intervalo de $\mathrm{pH}$ en que se realizó el estudio fue de $0-7$, los valores requeridos de $\mathrm{pH}$ fueron ajustados con solución de $\mathrm{HNO}_{3} 1,0 \mathrm{~mol} \mathrm{~L}^{-1}$ y adicionando solución de $\mathrm{KOH}$ 1,0 mol L $\mathrm{m}^{-1}$, los resultados son mostrados en la Figura 3.7. Como puede observarse, el potencial permanece constante a pH bajos con un comportamiento similar a lo observado en otro tipo de ESI ${ }^{104,105}$. El cambio de potencial a un $\mathrm{pH}$ arriba de 5, puede deberse a la formación de hidroxocomplejos con 
$\mathrm{Hg}$ (II). Así, el intervalo de 0 - 5 puede ser tomado como el intervalo de $\mathrm{pH}$ de trabajo del sensor propuesto ya que en este intervalo el sensor no responde a los iones $\mathrm{H}^{+}$y además concuerda con los estudios previos mostrados por Pérez-Gárcia et. al. ${ }^{106}$, donde las pruebas empíricas demuestran las formación del complejo Hg-PenDTF a pH’s ácidos.

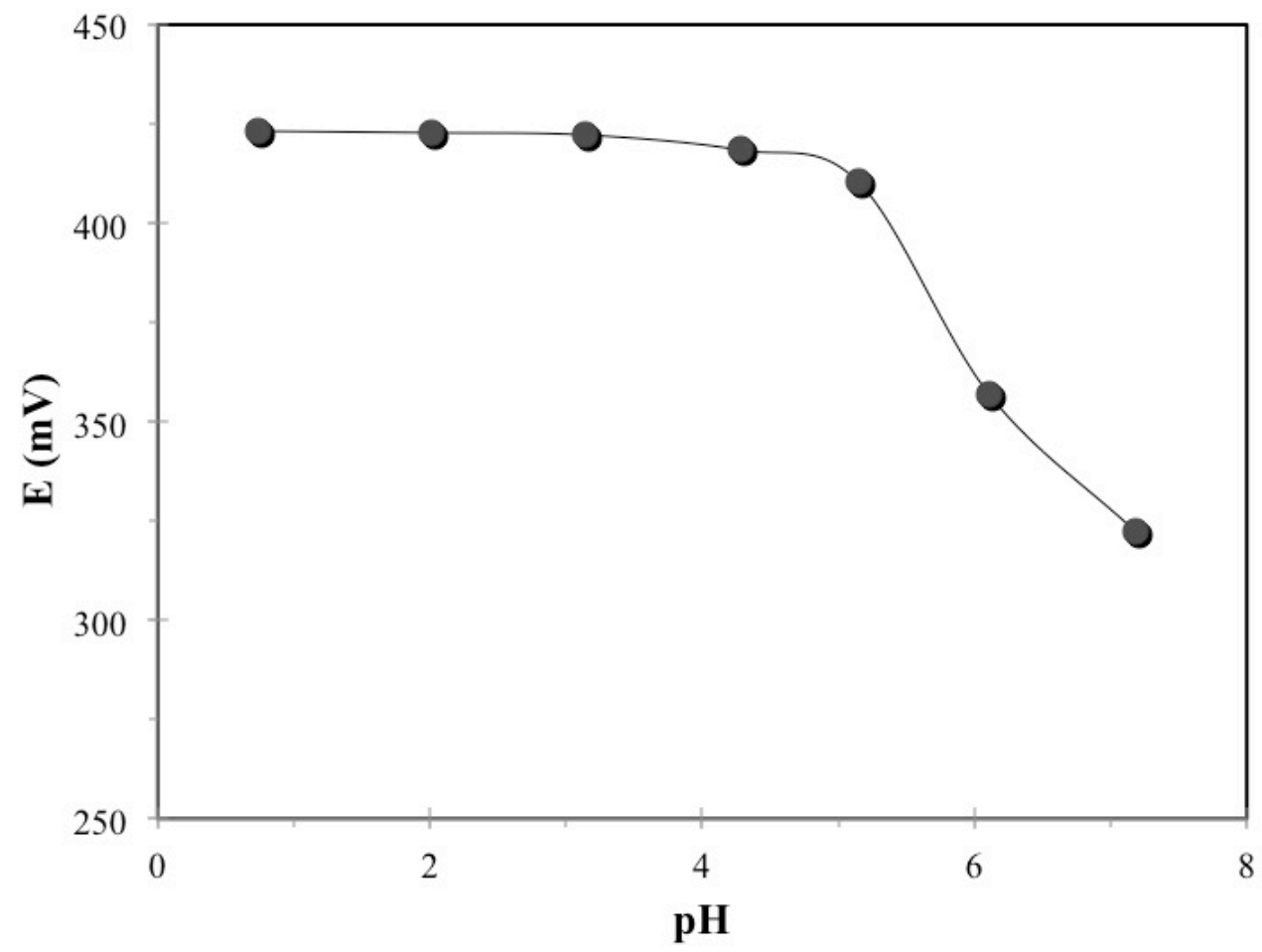

Figura 3.7. Efecto del $\mathrm{pH}$ sobre el electrodo selectivo a iones $\mathrm{Hg}(\mathrm{II})$ 


\subsubsection{SENSIBILIDAD REDOX PARA EL SENSOR Hg(II)-ISE-PenDTF 1}

Para descartar cualquier interferencia debida a una posible sensibilidad redox el electrodo Hg(II)-ISE-PenDTF 1 se estudio mediante voltamperometría cíclica. Se inicio en el potencial de circuito abierto yendo hacia potenciales positivos y se invirtió a potenciales negativos a una velocidad de $100 \mathrm{mV} \mathrm{s}^{-1}$ en solución de $\mathrm{HClO}_{4} 1.0 \mathrm{~mol} \mathrm{~L}^{-1}$ y $\mathrm{Hg}(\mathrm{II}) 2.0 \times 10^{-3} \mathrm{~mol} \mathrm{~L}^{-1}$. Los resultados se muestran en la figura 3.8. En el primer sistema (línea punteada) se utilizó como electrodo de trabajo un electrodo que no contiene al ionóforo PenDTF, en el segundo (línea continua) se utilizó el electrodo $\mathrm{Hg}$ (II)-ISE-PenDTF 1. Se observa que el electrodo que no contenía ionóforo tiene un pico de oxidación a $550 \mathrm{mV}$ y un pico de reducción a -310 mV, que están asociados al procesos redox $\mathrm{Hg}^{2+} / \mathrm{Hg}^{0}$, también se observó que el electrodo $\mathrm{Hg}$ (II)-ISE-1 PenDTF no presentó ninguna señal que pueda ser atribuida a un proceso de este tipo, lo que muestra que el sensor que aquí se propone no tiene ninguna sensibilidad redox. 


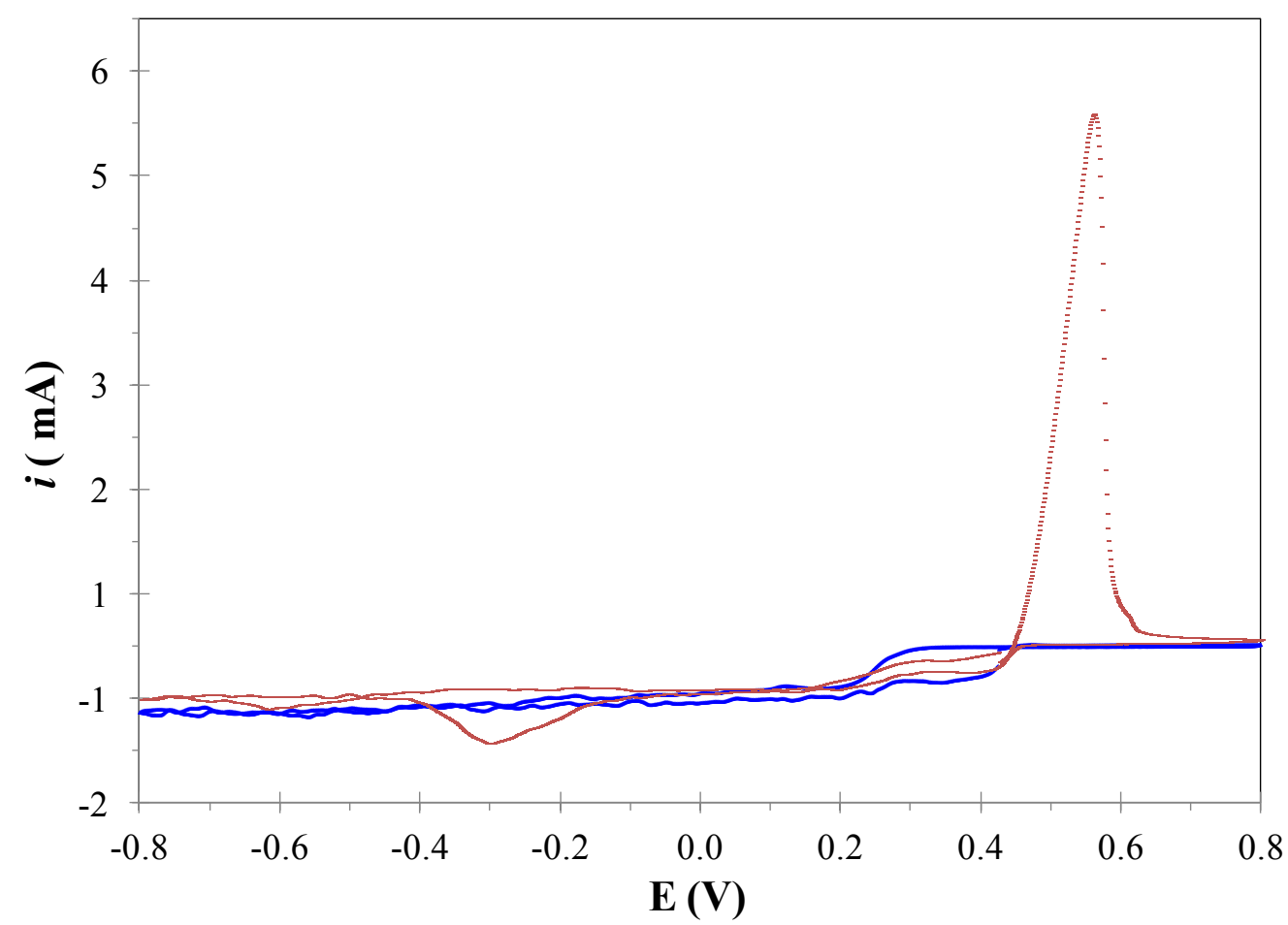

Figura 3.8. Voltamogramas cíclicos a $100 \mathrm{mV} \mathrm{s}^{-1}$ en $\mathrm{HClO}_{4} 1.0 \mathrm{~mol} \mathrm{~L}^{-1}$ y $\mathrm{Hg}$ (II) $2.0 \times 10^{-3} \mathrm{~mol} \mathrm{~L}^{-}$ 1. La línea de puntos, utilizando un electrodo sin PenDTF. Línea continua con el electrodo Hg (II)-ISE-PenDTF 1.

\subsubsection{SELECTIVIDAD DEL ELECTRODO}

La característica más importante de un electrodo selectivo a iones (ESI) es la capacidad de responder selectivamente a un ion primario en presencia de otros iones, este parámetro está directamente relacionado con el equilibrio termodinámico en la interfase superficie/solución. La selectividad del electrodo que contiene el ligante ditiofosforado PenDTF fue probado potenciométricamente con diferentes cationes divalentes como: $\mathrm{Ca}(\mathrm{II}), \mathrm{Ni}(\mathrm{II}), \mathrm{Cd}(\mathrm{II}), \mathrm{Mg}(\mathrm{II})$, $\mathrm{Cu}(\mathrm{II}), \mathrm{Co}(\mathrm{II}), \mathrm{Ba}(\mathrm{II}), \mathrm{y} \mathrm{Hg}(\mathrm{II})$, como puede verse en la Figura 3.9, la pendiente en la mayoría de 
los cationes probados muestra valores muy por debajo de lo esperado por la ecuación de Nernst, sin embargo para el $\mathrm{Hg}(\mathrm{II})$ se observa una respuesta potenciométrica lineal con un valor cercano al Nertstiano y en una amplio rango de concentración y bajo límite de detección.

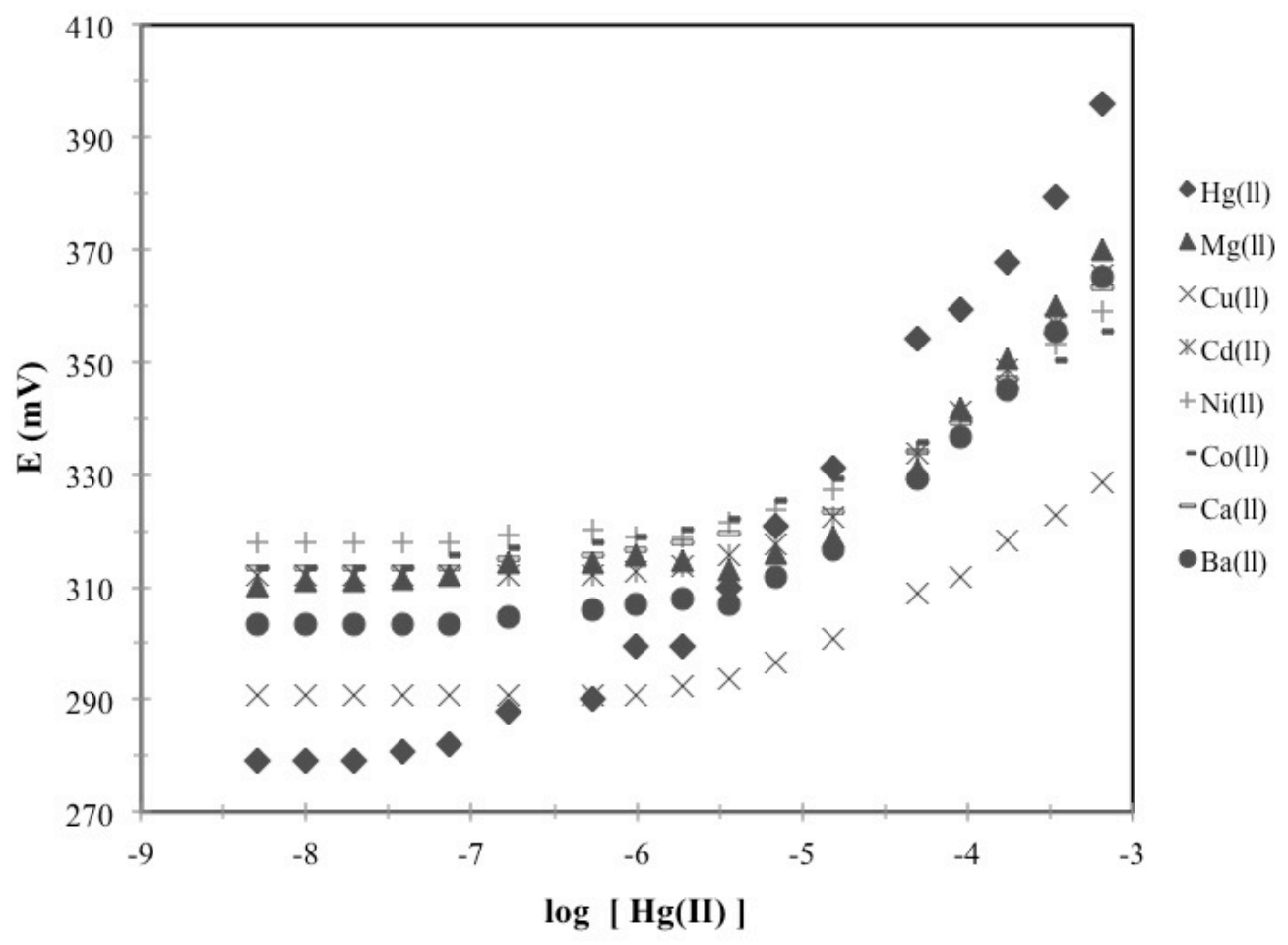

Figura 3.9. Respuesta potenciométrica del electrodo selectivo de iones basado en PenDTF para diferentes iones metálicos.

\subsubsection{COEFICIENTES DE SELECTIVIDAD}

El coeficiente de selectividad potenciométrico (que se mide en términos de la constante de selectividad portenciométrica $K_{i, j}^{P o t}$ ) que describe la preferencia del electrodo sugerido a un ión interferente en presencia de $\mathrm{Hg}$ (II) fue determinado mediante el método de interferencia fija el cual se basa en la ecuación semiempírica de Nikolsky-Eisenman ${ }^{107,108}$. 


$$
E_{I S E}=E^{0}+\frac{R T}{z_{i} F} \ln \left[a_{i}+\sum_{j} K_{i, j}^{p o t} a_{j}^{z_{i} / z_{j}}\right]
$$

Donde $\mathbf{E}_{\mathbf{I S E}}$ es el potencial medido, $\mathbf{E}^{\mathbf{0}}$ el potencial estándar, $\mathbf{a}_{\mathbf{i}} \mathbf{y} \mathbf{a}_{\mathbf{j}}$ las actividades de los iones primario e interferente respectivamente. En este método, la concentración del ion primario es variada en una solución $1.0 \mathrm{~mol} \mathrm{~L}{ }^{-1}$ de $\mathrm{HClO}_{4}$ y la concentración del ion secundario se mantiene constante en $1.0 \times 10^{-3} \mathrm{~mol} \mathrm{~L}{ }^{-1}$, las ecuaciones para cada ion se pueden escribir como:

$$
\begin{gathered}
E_{i}=E^{0}+\frac{R T}{Z_{i} F} \ln \left[a_{i}\right] \\
E_{j}=E^{0}+\frac{R T}{Z_{i} F} \ln \left[K_{i, j}^{p o t} a_{j}^{z_{i} / z_{j}}\right]
\end{gathered}
$$

Igualando potenciales se tiene: $\quad K_{i, j}^{p o t}=\frac{a_{i}}{{ }_{a_{j}}^{z_{i} / z_{j}}}$

De esta manera utilizando el método de interferencia fija, se calcularon los coeficientes de selectividad que se muestran en la Tabla 3.3. 
Tabla 3.3. Coeficiente de selectividad potenciométrica $K_{i, j}^{P o t}$ (método del interferente fijo).

\begin{tabular}{cc}
\hline Ion interferente & $\left(K_{H g(I I), j}^{P o t}\right)$ \\
\hline $\mathrm{Ba}(\mathrm{II})$ & $(2.56 \pm 0.24) \times 10^{-5}$ \\
$\mathrm{Ca}(\mathrm{II})$ & $(6.29 \pm 0.55) \times 10^{-5}$ \\
$\mathrm{Co}(\mathrm{II})$ & $(1.35 \pm 0.15) \times 10^{-6}$ \\
$\mathrm{Mg}(\mathrm{II})$ & $(1.96 \pm 0.27) \times 10^{-4}$ \\
$\mathrm{Cu}(\mathrm{II})$ & $(1.13 \pm 0.84) \times 10^{-6}$ \\
$\mathrm{Cd}(\mathrm{II})$ & $(4.77 \pm 0.29) \times 10^{-5}$ \\
$\mathrm{Ni}(\mathrm{II})$ & $(5.44 \pm 0.53) \times 10^{-5}$ \\
\hline
\end{tabular}

De las gráficas de $\mathrm{E}_{\mathrm{ISE}}$ vs. $\log \left[\mathrm{Hg}^{2+}\right]$ y con el uso de la expresión anterior para $K_{i, j}^{\text {Pot }}$ se determinaron los coeficientes de selectividad potenciométrica para distintos cationes metálicos. Como puede verse en la mayoría de los cationes utilizados, los valores de coeficientes de selectividad son menores a 0.01 , lo que indica que la interferencia producida por estos iones en el funcionamiento del sensor es despreciable. La secuencia de selectividad que el ESI basado en PenDTF tiene hacia los diferentes iones obedece al orden: $\mathrm{Hg}($ II $)>\mathrm{Mg}(\mathrm{II})>\mathrm{Ca}(\mathrm{II})>\mathrm{Ni}(\mathrm{II})>$ $\mathrm{Cd}(\mathrm{II})>\mathrm{Ba}(\mathrm{II})>\mathrm{Cu}(\mathrm{II})>\mathrm{Co}(\mathrm{II})$.

\subsubsection{APLICACIÓN ANALÍTICA}

\subsubsection{VALORACIÓN POTENCIOMÉTRICA}

El ISE se usó como electrodo indicador en la valoración potenciométrica de $10 \mathrm{ml}$ de una solución de $\mathrm{Hg}\left(\mathrm{NO}_{3}\right)_{2} 1.0 \times 10^{-3} \mathrm{~mol} \mathrm{~L}^{-1}$ con EDTA $1.0 \times 10^{-2} \mathrm{~mol} \mathrm{~L}^{-1}$ a pH 0 y 4 impuesto con 
$\mathrm{HClO}_{4}$, las curvas de valoración se presentan en la Figura 3.10. En esta figura se puede observar que el punto de inflexión no es muy prolongado, lo que implica que la reacción de valoración mercurio + EDTA $=$ mercurioEDTA no es muy cuantitativa.

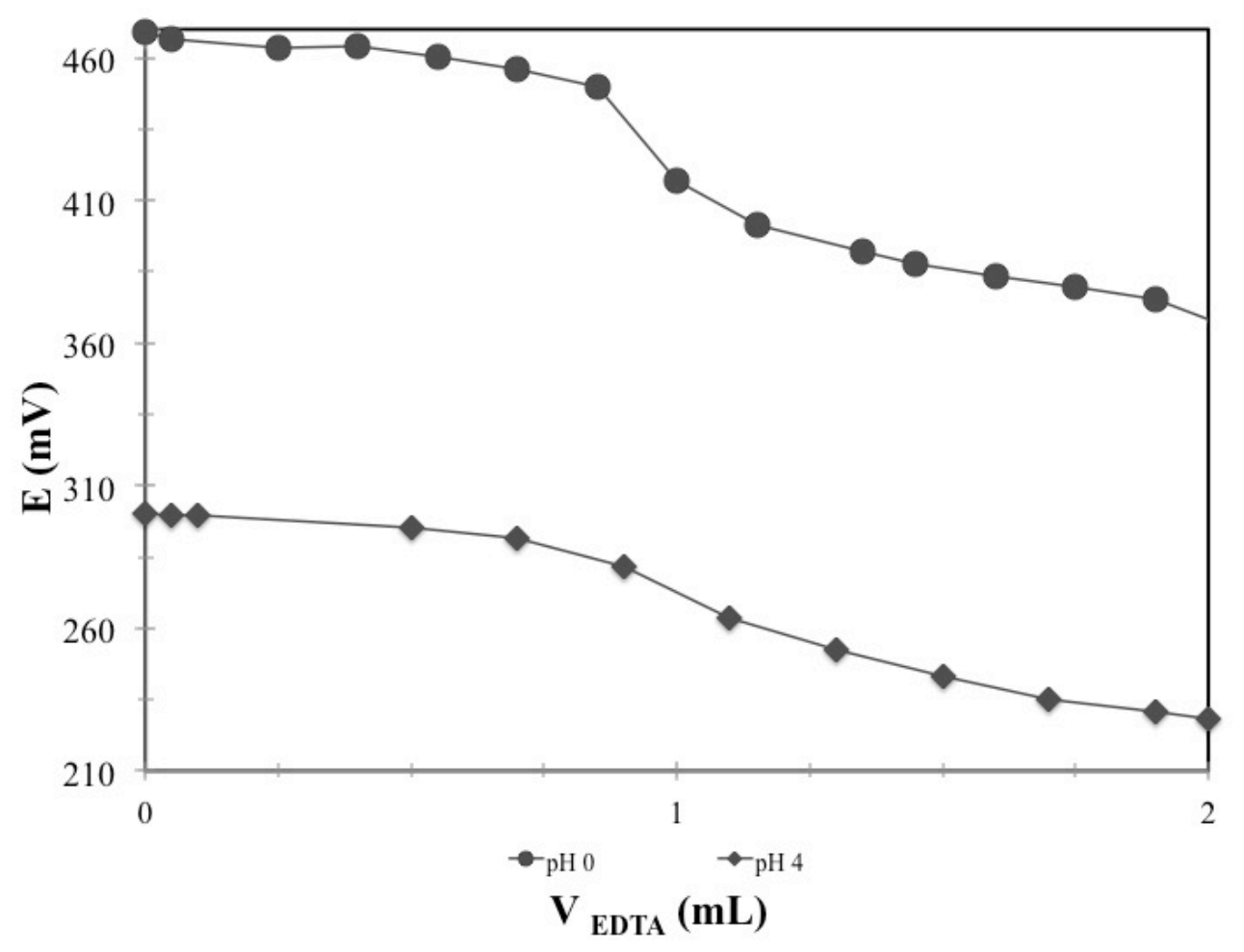

Figura 3.10. Curvas de valoración portenciométrica de $10 \mathrm{~mL}$ de una solución de $\mathrm{Hg}^{2+} \quad 1.0$ x $10^{-3} \mathrm{~mol} \mathrm{~L}^{-1}$ con EDTA $1.0 \times 10^{-2} \mathrm{~mol} \mathrm{~L}^{-1}$ a pH's $0(\bullet)$ y $4(\bullet)$.

En la Figura 3.11 se muestran los diagramas de zonas de predominio (DZP) y de existencia predominio (DEP) para el sistema $\mathrm{Hg}(\mathrm{II}) / \mathrm{H}_{2} \mathrm{O}$. En las cuales se observan las especies participantes en las reacción de valoración, a pH 0, se concluye q la reacción de valoración es: $\mathrm{Hg}^{2+}+\mathrm{H}_{4} \mathrm{Y}=\mathrm{HgYH}^{-}+3 \mathrm{H}^{+}$con un $\log \mathrm{Kr}$ ' $=3.5 \mathrm{y}$ como para que una reacción de este tipo sea cuantitativa $\mathrm{K}_{\mathrm{r}}{ }^{\prime} \mathrm{C}_{\mathrm{o}} \geq 10^{5}$. Como en este caso $\mathrm{K}_{\mathrm{r}}{ }^{\prime} \mathrm{C}_{\mathrm{o}}=10^{0.5}$, la reacción no es cuantitativa. 

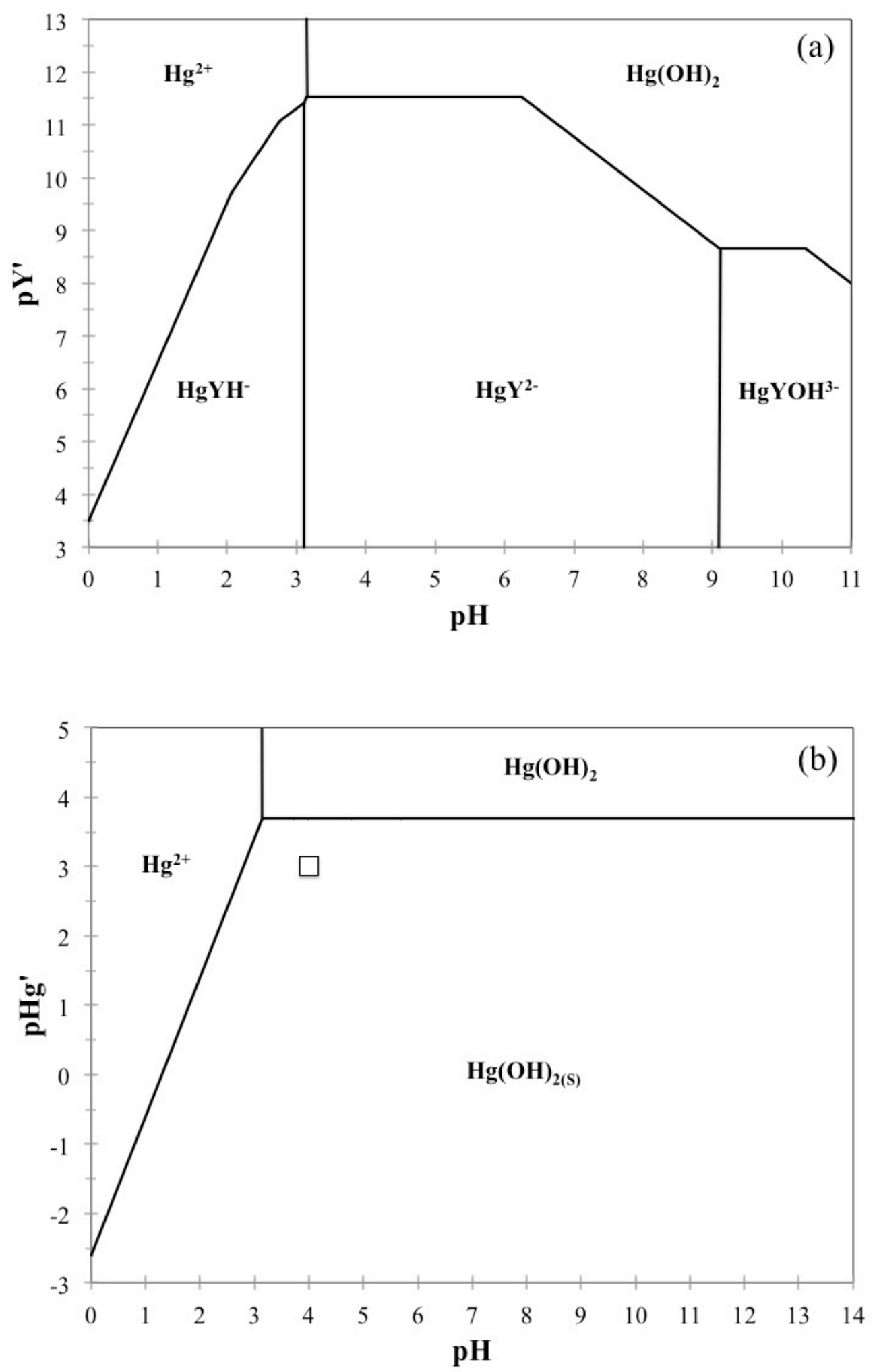


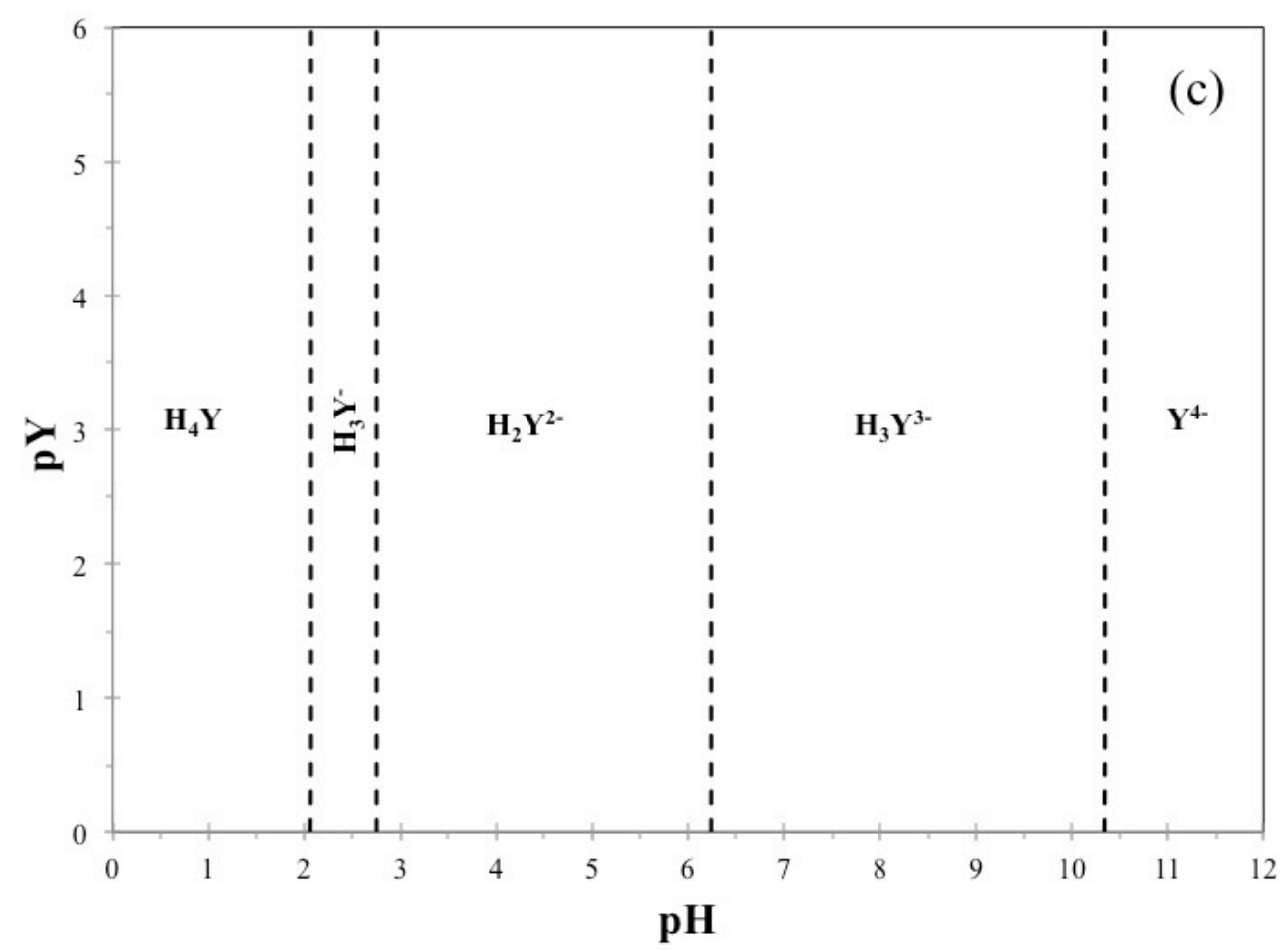

Figura 3.11. (a) Diagrama de zonas de predominio (DZP) para el sistema $\mathrm{Hg}(\mathrm{II}) / \mathrm{H}_{2} \mathrm{O}$; (b) Diagrama de exixtencia predominio (DEP) para $\mathrm{Hg}(\mathrm{II})$, el símbolo $\square$ representa el punto (pHg', $\mathrm{pH})=(3,4)$ y (c) DZP para las especies de EDTA.

A pH 4, la reacción de valoración es: $\mathrm{Hg}(\mathrm{OH})_{2}+\mathrm{H}_{2} \mathrm{Y}^{2-}=\mathrm{HgY}^{2-}+2 \mathrm{H}_{2} \mathrm{O}$ la cual tiene un $\log \mathrm{Kr}^{\prime}=11.52$ cuando se tratan a las especies en solución, pero la Figura 3.11 (b) demuestra la presencia de la especie insoluble hidróxido de mercurio, lo cual desfavorece la reacción de valoración y abate la cuantitatividad de la reacción.

Se analizó el sistema $\mathrm{Hg}(\mathrm{II}) / \mathrm{Ac} / \mathrm{H}_{2} \mathrm{O}$ para establecer las condiciones óptimas para el buen funcionamiento del sensor y para garantizar la cuantitatividad de la reacción de valoración de mercurio con EDTA. En la Figura 3.12 se presentan los DZP de las especies generalizadas a 
segundo orden $\mathrm{Hg}^{\prime}$ ' y $\mathrm{HgY}^{\prime}$, para el sistema $\mathrm{Hg}(\mathrm{II}) / \mathrm{Ac} / \mathrm{H}_{2} \mathrm{O}$, la línea horizontal en (a) indica el amortiguamiento a primer orden $\left(\mathrm{pAc}^{\prime}=3,0\right)$.

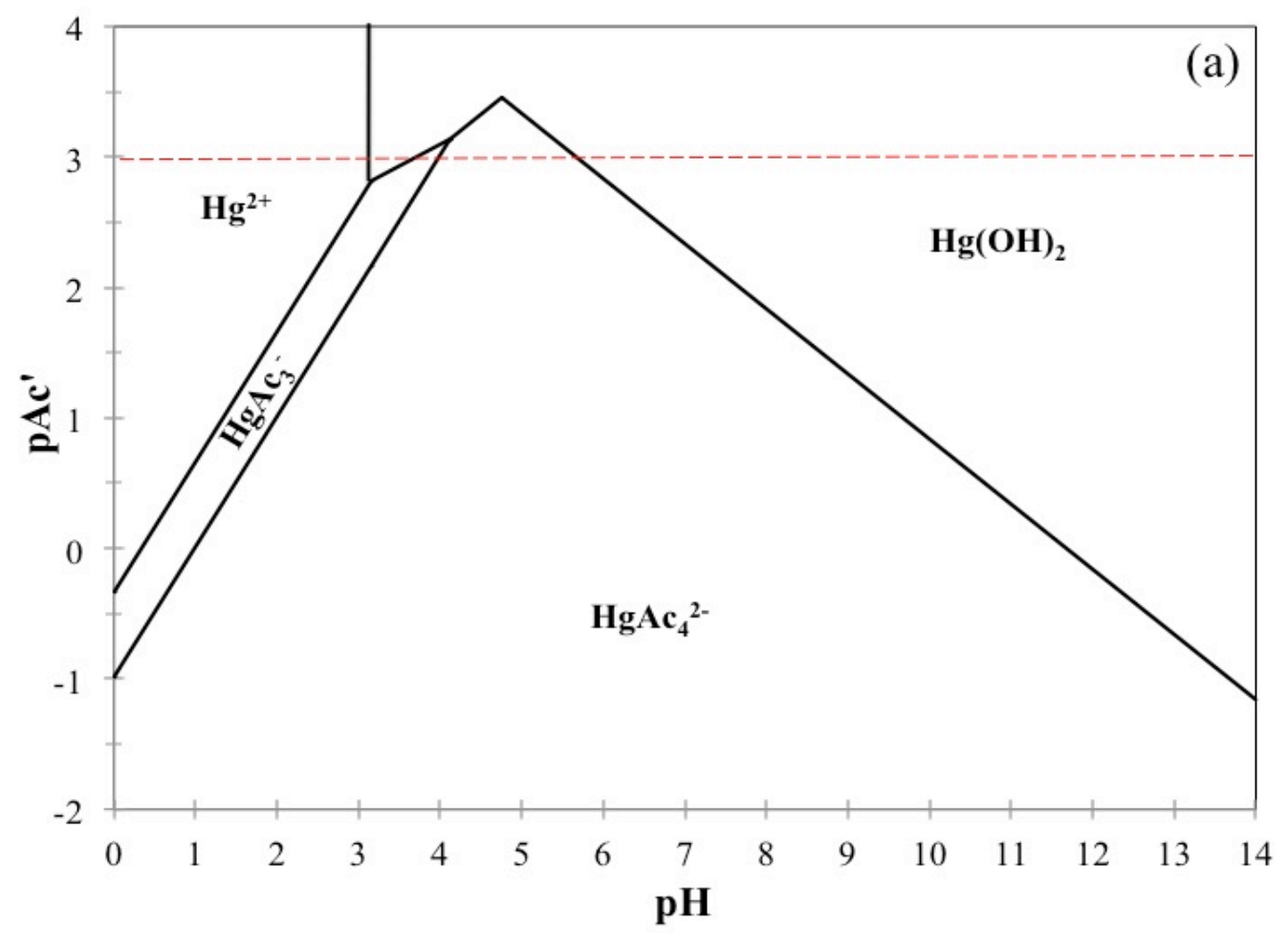




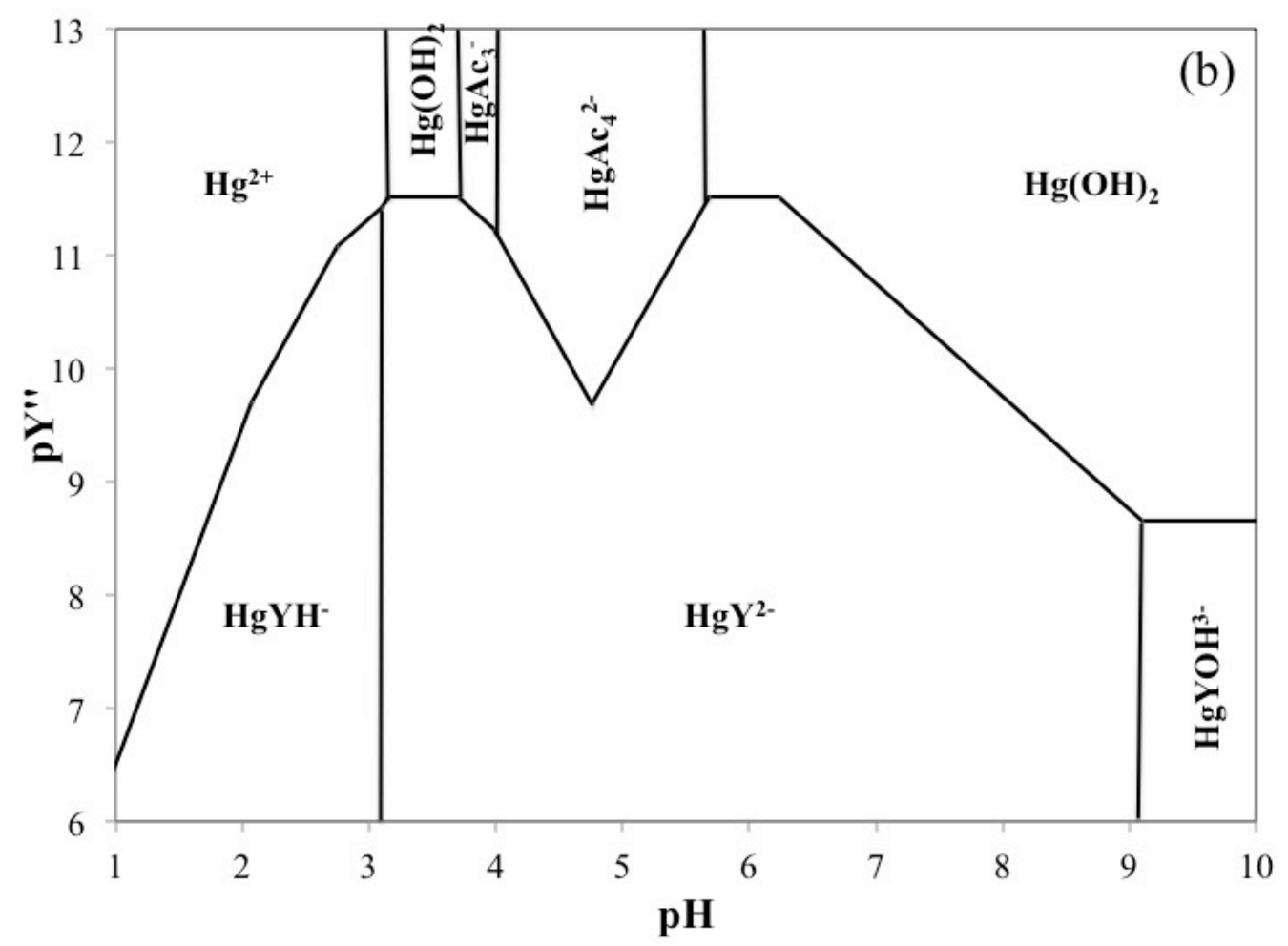

Figura 3.12. Diagramas de zonas de predominio (DZP) para el sistema $\mathrm{Hg}(\mathrm{II}) / \mathrm{Ac} / \mathrm{H}_{2} \mathrm{O}$. (a) $\mathrm{DZP}$ para $\mathrm{Hg}^{\prime}$; (b) DZP para $\mathrm{HgY}^{\prime \prime}$ a pAc' = 3,0.

En la Figura 3.13 se presenta el DEP de las especies de $\mathrm{Hg}(\mathrm{II})$. Estos diagramas se construyeron con la metodología descrita por Rojas-Hernández et. al. ${ }^{109,110}$. De este diagrama se concluye que el hidróxido de mercurio no precipita para un $\mathrm{pHg} "=3$ cuando se ha impuesto el $\mathrm{pH}$ del sistema en 5.0 con un amortiguador de $\mathrm{AcOH} / \mathrm{NaAcO} 0.01 \mathrm{~mol} \mathrm{~L}^{-1}$. 


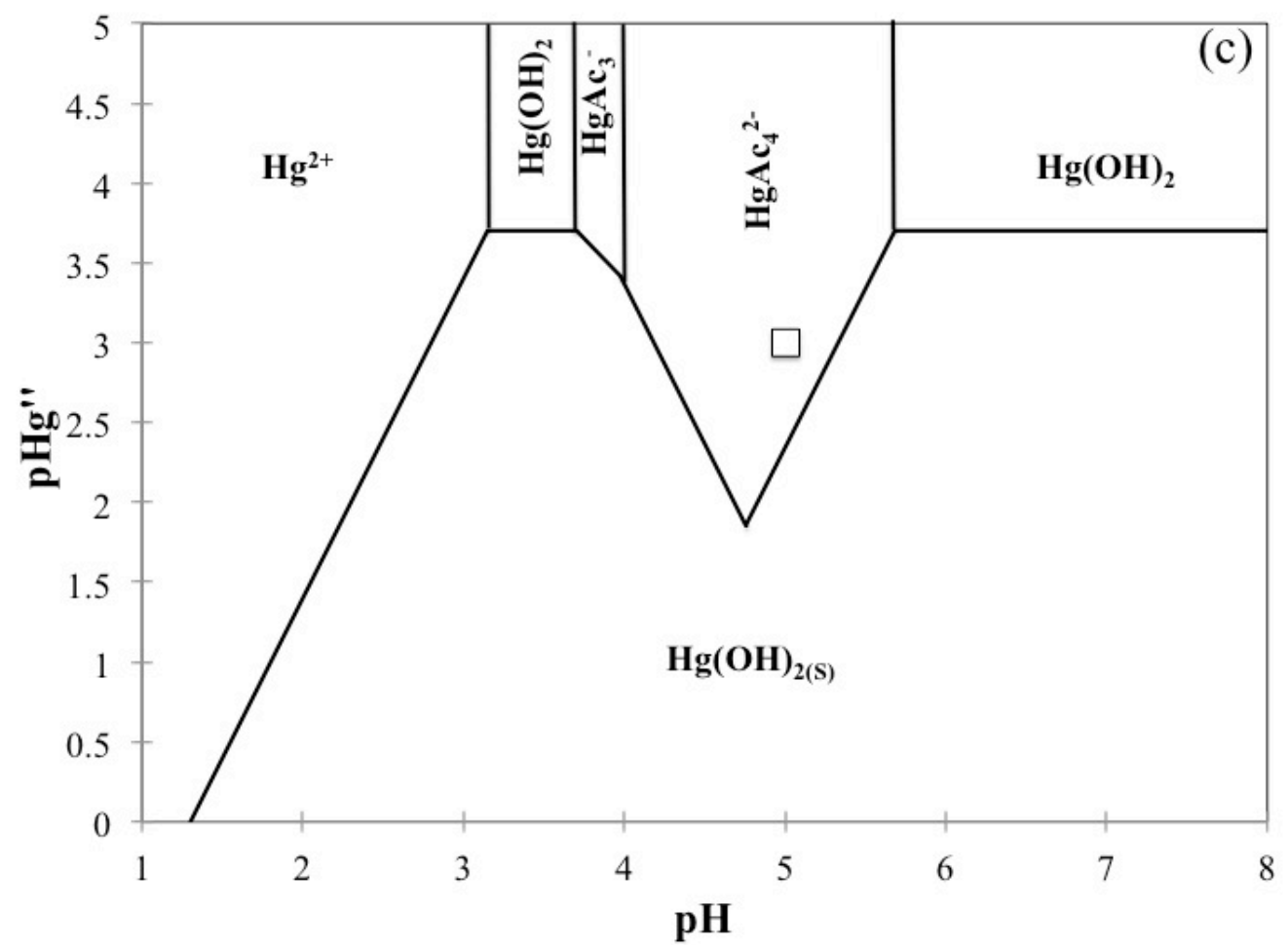

Figura 3.13. Diagrama de existencia-predominio (DEP) para el sistema $\mathrm{Hg}(\mathrm{II}) / \mathrm{Ac} / \mathrm{H}_{2} \mathrm{O}$ a pAc' $=$ 3, el símbolo $\square$ representa el punto $\left(\mathrm{pHg}{ }^{\prime}, \mathrm{pH}\right)=(3,5)$.

De las Figuras 3.11, 3.12 y 3.13 se concluye que $\mathrm{Y}^{\prime \prime}=\mathrm{H}_{2} \mathrm{Y}^{2-}, \mathrm{Hg}{ }^{\prime \prime}=\mathrm{HgAc}_{4}{ }^{2-}$ y $\mathrm{HgY}{ }^{\prime \prime}=\mathrm{HgY}^{2-}$ . De esta forma la reacción de valoración representativa del $\mathrm{Hg}(\mathrm{II})$ con EDTA a pH 5.0 impuesto con amortiguador de $\mathrm{AcOH} / \mathrm{NaAcO} 0.01 \mathrm{~mol} \mathrm{~L}^{-1}$ es:

$$
\mathrm{HgAc}_{4}^{2-}+\mathrm{H}_{2} \mathrm{Y}^{2-}=\mathrm{HgY}^{2-}+4 \mathrm{Ac}^{-}+2 \mathrm{H}^{+}
$$

y se tiene que: $\log K_{r}^{\prime \prime}=-11.84+4 p A c^{\prime}+2 p H=10.16$ y como en este caso $\mathrm{K}_{\mathrm{r}}{ }^{\prime} \mathrm{C}_{\mathrm{o}}=10^{7.16}$, la reacción de cuantitativa.

Los resultados de la titulación de $\mathrm{Hg}$ (II) se muestran en la Figura 3.14. El valorante añadido causó una disminución en el potencial como resultado de una disminución en la concentración 
del ion $\mathrm{Hg}$ (II) debido a la formación de un complejo con EDTA. La cantidad de iones $\mathrm{Hg}$ (II) en solución puede ser determinada con precisión a partir de las curvas de valoración resultantes, de la curva se obtiene que el volumen en el punto de equivalencia es $0.97 \pm 6.0 \times 10^{-3} \mathrm{~mL}$ con lo cual se obtiene una concentración de $\mathrm{Hg}$ (II) de $9.7 \times 10^{-4} \pm 7.0 \times 10^{-5} \mathrm{M}$ y un porcentaje de recobro del $97 \pm 7 \%$.

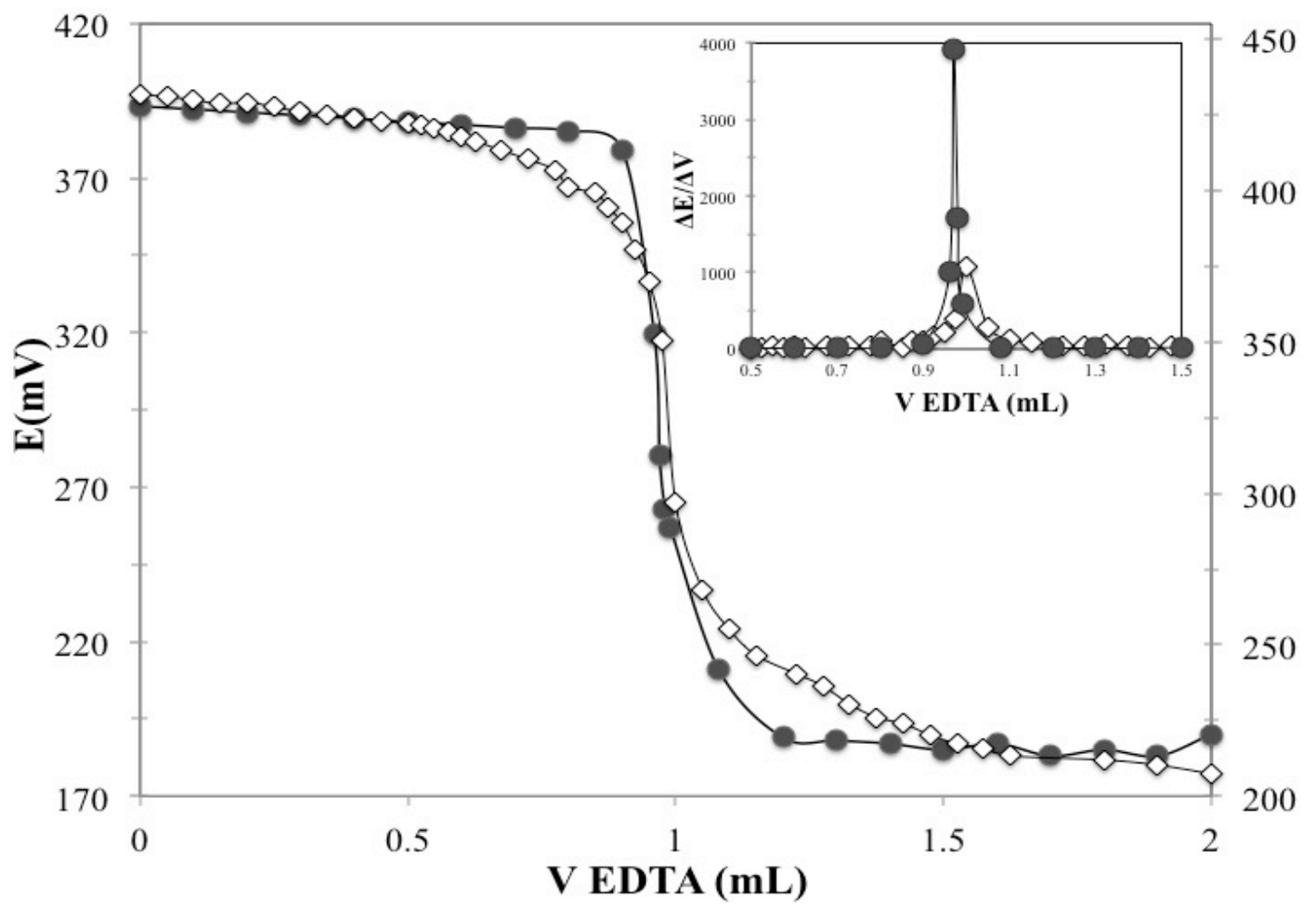

Figura 3.14. Curva de valoración portenciométrica de $10 \mathrm{ml}$ de una solución de $\mathrm{Hg}^{2+} \quad 1.0 \times 10^{-3}$ mol L ${ }^{-1}$ con EDTA $1,0 \times 10^{-2} \mathrm{~mol} \mathrm{~L}^{-1} .\left(^{\bullet}\right)$ utilizando el sensor CE_DTF-1 y ( $\square$ ) usando una "J" de mercurio comercial. En pernspectiva, la primera derivada de las curvas de valoración.

Adicionalmente, en la Figura 3.14 se muestra la curva de titulación de la misma concentración de $\mathrm{Hg}(\mathrm{II})$ y en las mismas condiciones utilizando un electrodo comercial de gota de mercurio, se 
puede observar en la superposición de las curvas la semejanza de estas y del punto de inflexión, lo cual indica que el sensor aquí propuesto puede utilizarse con veracidad en la valoracion de iones $\mathrm{Hg}(\mathrm{II})$.

\subsubsection{COMPARACIÓN CON LA LITERATURA}

En la Tabla 3.4, se presentan algunos electrodos selectivos a $\mathrm{Hg}(\mathrm{II})$ encontrados en la literatura $111,112,113,114,115,116,117,118,119,120$, en ella se pueden observar el tipo de compuesto utilizado como elemento de reconocimiento, la configuración del electrodo y algunos parámetros analíticos como son: su respuesta Nernstiana así como el intervalo de trabajo, con el fin de comparar con el aquí reportado. El sensor propuesto en este trabajo, presenta algunas ventajas, tanto en la construcción, en el funcionamiento, así como en la síntesis del elemento de reconocimiento. Al ser un electrodo de contacto interno sólido se eliminan el electrodo de referencia interno y también la solución de referencia, facilitando así la construcción, mantenimiento y manejo.

El sensor presenta otra ventaja con respecto a los reportados que se basan en membranas poliméricas, ya que componentes como un plastificante y un intercambiador iónico son esenciales para la preparación del coctel polimérico, otras ventajas que presenta este electrodo frente a los basados en membranas poliméricas es que si la membrana sufriera algún daño o se contaminara, no se podría regenerar fácilmente y el aquí reportado tiene la propiedad que si por alguna razón los sitios activos del sensor en la superficie se bloquearan, esté se podría regenerar solo lijando la superficie y se obtendrían sitios activos nuevos y disponibles. El ionóforo utilizado en este trabajo es aproximadamente 60 veces menos costoso de obtener frente a algunos de membrana polimérica ${ }^{111,114-119}$ y estos sensores no presentan mejores parámetros analíticos. 
Frente a dispositivos reportados que no se basan en mediciones potenciométricas, también presenta sus ventajas ya que la síntesis de compuestos cromosensores, se extienden las rutas de síntesis y también se eleva el costo de preparación de los compuestos.

\subsection{CONCLUSIONES}

El electrodo basado en O,O'-(2,2'-bifenilen)ditiofosfato de pentilo (PenDTF) mostró una buena respuesta Nernstiana a $\operatorname{Hg}(\mathrm{II})$ (aún en presencia de iones metálicos divalentes) en un amplio rango de concentración. El intervalo de pH aplicable, el límite inferior de detección, y los coeficientes de selectividad potenciométricos del sensor propuesto, indican que es un dispositivo comparable con otros métodos utilizados para la determinación de este ion y que presenta algunas ventajas frente a otros sensores propuestos en la literatura. El sensor basado en PenDTF muestra diferentes ventajas desde el costo de la síntesis del ionóforo, como su diseño de contacto interno sólido renovable que puede ser utilizado para varios experimentos con solo tratamiento con papel abrasivo, la eliminación de la solución interna de referencia hace que no se necesite mantenimiento y favorece su almacenamiento. 
Tabla 3.4. Recientes electrodos selectivos de iones para $\mathrm{Hg}(\mathrm{II})$ propuestos en la literatura.

\begin{tabular}{|c|c|c|c|c|c|c|}
\hline Ref. & Ionóforo & $\begin{array}{l}\text { Tipo del } \\
\text { ISE }\end{array}$ & $\begin{array}{l}\text { Pendiente } \\
(\mathrm{mV} \\
\left.\text { decade }^{-1}\right) \\
\end{array}$ & $\begin{array}{c}\text { Intervalo } \\
\text { lineal } \\
\left(\mathrm{mol} \mathrm{L}^{-1}\right) \\
\end{array}$ & $\begin{array}{l}\text { Rango } \\
\text { de } \mathrm{pH}\end{array}$ & Selectividad $\left(K_{H g(I I), j}^{P o t}\right)$ \\
\hline [111] & Dithiophosphorus macrocyclic compound & Membrana & 30.2 & $\begin{array}{l}1.0 \times 10^{-} \\
6-1.0 \times\end{array}$ & $\begin{array}{c}3.0- \\
6.0\end{array}$ & $\begin{array}{lr}\mathrm{Pb}^{2+} ; \quad 6.4 \times 10^{-2}, & \mathrm{Cd}^{2+} ; \\
6.2 \times 10^{-3}, \mathrm{Ni}^{2+} ; & 4.2 \times 10^{-3}, \\
\mathrm{Co}^{2+} ; 6.9 \times 10^{-3}, & \mathrm{Mg}^{2+} ;\end{array}$ \\
\hline [112] & Dibenzyl calix[4]arene-dithiacrown & Membrana & 29.5 & $\begin{array}{l}3.0 \times 10^{-} \\
7-3.0 \times \\
10^{-2}\end{array}$ & ----- &  \\
\hline [113] & $\begin{array}{l}8,17 \text {-bis(pyren-1-ylmethyl)-6,7,8,9,15,16,17,18- } \\
\text { octahydrodibenzo }[f, m][1,8,4,11] \text { dithiodiazacyclotetradecine }\end{array}$ & Membrana & 27.6 & $\begin{array}{c}1.0 \times 10^{-} \\
6-1.0 \times \\
10^{-2}\end{array}$ & $\begin{array}{l}4.0- \\
4.5\end{array}$ & 10 - \\
\hline [114] & Benzothiazole azocalix[4] arene (BTC4) & $\begin{array}{l}\text { Pasta de } \\
\text { carbono }\end{array}$ & ----- & $\begin{array}{c}1.0 \times 10^{-} \\
4-1.0 \times \\
10^{-2}\end{array}$ & ----- & ----- \\
\hline$[115]$ & $\begin{array}{l}\text { 26,27,28-tris-benzyloxy-25-hidroxy-5,11,17,23-tetra-tert- } \\
\text { butyl-calix[4]arene }\end{array}$ & Membrana & 29.4 & $\begin{array}{l}1.0 \times 10^{-} \\
6-1.0 \times \\
10^{-1}\end{array}$ & $\begin{array}{l}1.5- \\
4.0\end{array}$ & $\begin{array}{l}\mathrm{Ca}^{2+} ; \quad 5.3 \times 10^{-3}, \quad \mathrm{Sr}^{2+} ; \\
3.8 \times 10^{-4}, \mathrm{Ba}^{2+} ; 1.9 \times 10^{-3}, \\
\mathrm{Co}^{2+} ; \quad 3.5 \times 10^{-3}, \quad \mathrm{Zn}^{2+} ; \\
1.3 \times 10^{-3}, \mathrm{~Pb}^{2+} ; 5.1 \times 10^{-3},\end{array}$ \\
\hline [116] & $\begin{array}{l}\text { N,N'-bis(pyridin-2-ylmethyl)-p-tert-butylcalix[4]arene- } \\
\text { diaza-crown }\end{array}$ & Membrana & $\begin{array}{l}-27.8,- \\
52.7\end{array}$ & $\begin{array}{c}1.0 \times 10^{-} \\
6-1.0 \times \\
10^{-2}\end{array}$ & ----- & $\lambda^{2+} \cdot \begin{array}{c}1 \\
7 \mathbf{v} 1 n^{-3} \\
----\end{array}$ \\
\hline$[117]$ & N,N'-bis(salicylaldehyde)-phenylendiamine & Membrana & 58.8 & $\begin{array}{c}3.2 \times 10^{-} \\
7-3.2 \times \\
10^{-4}\end{array}$ & $\begin{array}{l}3.8- \\
7.8\end{array}$ & 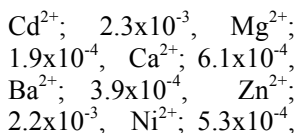 \\
\hline [118] & $\begin{array}{l}\text { 1-furan-2-yl-4-(4-nitrophenyl)-2-phenyl-5H-imidazole-3- } \\
\text { oxide }\end{array}$ & Membrana & 29.3 & $\begin{array}{l}1.0 \times 10^{-} \\
5-1.0 \times \\
10^{-1}\end{array}$ & $\begin{array}{c}1.0- \\
4.0\end{array}$ & ----- \\
\hline$[119]$ & 1,3,bis(2-methoxybenzene)triazene (MBT) & Membrana & 30.2 & $\begin{array}{l}1.0 \times 10^{-} \\
7-1.0 \times \\
10^{-2}\end{array}$ & $\begin{array}{l}2.6- \\
4.2\end{array}$ & $\begin{array}{l}\mathrm{Zn}^{2+} ; \quad 1.8 \times 10^{-3}, \\
3.8 \times 10^{-3}, \mathrm{~Pb}^{2+} \\
\mathrm{Cd}^{2+} ; \quad 5.8 \times 10^{-3}, \\
2.2 \times 10^{-5}\end{array}$ \\
\hline$[120]$ & Oxadiazaphosphepina derivatives & Membrana & 29.4 & $\begin{array}{l}1.0 \times 10^{-} \\
{ }^{6}-1.0 \times \\
10^{-1}\end{array}$ & $\begin{array}{c}0.9- \\
4.5\end{array}$ & ----- \\
\hline $\begin{array}{c}\text { This } \\
\text { work }\end{array}$ & O,O'-(2,2'-bifenylen)dithiophosphate pentyl & $\begin{array}{c}\text { Carbón- } \\
\text { epoxy }\end{array}$ & 33.8 & $\begin{array}{c}5.3 \times 10^{-} \\
7-1.0 \times \\
10^{-2}\end{array}$ & $\begin{array}{c}0.0- \\
5.0\end{array}$ & Tabla 3.3 \\
\hline
\end{tabular}




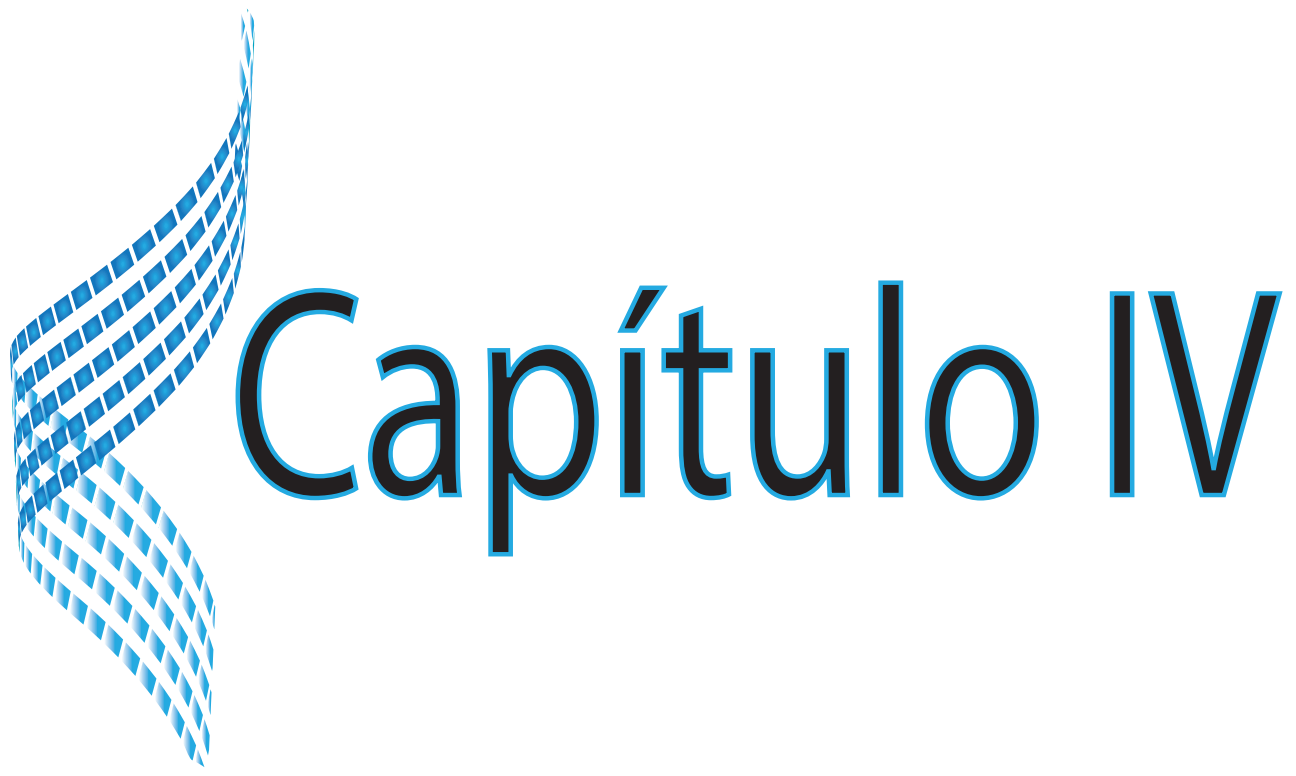

Enseñar no es una función vital, porque no tiene el fin en sí misma; la función vital es aprender. Aristóteles 


\section{Capítulo IV}

\section{CAPÍTULO IV. Nuevo Electrodo Potenciométrico de Contacto Interno Sólido para la Detección Selectiva de Mercurio a Niveles Nanomolares}

\subsection{INTRODUCCIÓN}

El mercurio es uno de los elementos más tóxicos y el medio ambiente (ríos, mares, etc) ha sido y sigue siendo el lugar donde numerosas empresas desechan enormes cantidades de este elemento en sus distintas formas (elemental, orgánica e inorgánica) sin importar los efectos que este hecho acarree. El Biodiversity Research Institute en colaboración con el IPEN (International POPs Elimination Network) publicó en enero del 2013 una investigación realizada en nueve países sobre los niveles de mercurio en muestras de pescado y cabello de la población ${ }^{121}$, arrojando como resultado que el pescado de los nueve países tenían una elevada concentración de metilmercurio que superaba la normativa de $0.22 \mathrm{ppm}$ dictada por la US EPA (Unied States 
Environmental Protection Agency) y países como Japón y Uruguay superan la norma de 1.0 ppm de la Organización Mundial de la Salud, los estudios en muestras de cabello arrojaron que más del $82 \%$ de las personas examinadas tenían concentraciones de mercurio superiores a $1.0 \mathrm{ppm}$.

Los compuestos de mercurio son ampliamente utilizados en la industria para la fabricación de pilas y baterías, lámparas fluorescentes, instrumentos de medición, amalgamas dentales, plaguicidas, aditivos de pinturas, papel, cosméticos y ungüentos de uso tópico ${ }^{122,123,124}$.

La prolongada exposición a compuestos derivados de este metal desemboca en numerosos problemas tales como: disminución de la habilidad cognitiva, alteraciones del sueño y hasta envenenamiento por la inhalación crónica de vapores de mercurio elemental, ya que al inhalarlo el mercurio elemental es conducido por el sistema respiratorio y distribuido por todo el cuerpo por el sistema circulatorio absorbiéndose en los tejidos biológicos por efecto de la fuerte interacción que tiene este metal hacia los grupos donadores de azufre ${ }^{125,126}$ presentes en la molécula de cisteína (aminoácido de numerosas estructuras proteínicas). Por su parte, la sales inorgánicas de mercurio pueden ser altamente toxicas y corrosivas, una alta exposición a estas sales puede llevar a problemas de corrosión graves en el estómago, intestinos, riñón y otros órganos, hasta el sistema nervioso central podría sufrir daños ${ }^{127}$. Por si esto fuera poco, las formas elemental e inorgánicas de mercurio pueden ser biotransformadas por microorganismos a formas orgánicas, tales como metilmercurio y fenilmercurio. Se cree que el metilmercurio es aun más tóxico que la forma elemental ya que puede causar fuertes daños neurológicos, presión arterial alta, problemas cardiacos y trastornos en el riñón. 
Los métodos clásicos que se han utilizado en la determinación de mercurio y que son caracterizados por tener un bajo límite de detección son: la espectroscopía de absorción atómica (AAS) con límites de detección alrededor de $1.0 \times 10^{-11} \mathrm{~mol} \mathrm{~L}^{-1},{ }^{128}$ la espectrometría de masas con plasma de acoplamiento inductivo (ICP-MS), ${ }^{129}$ la electroforesis capilar con límites de detección alrededor de $1.0 \times 10^{-8} \mathrm{~mol} \mathrm{~L}^{-1}{ }^{130}$ entre otras, pero son técnicas de alto costo que no pueden ser utilizadas en análisis de campo. Entre las diferentes técnicas para el análisis de iones metales, el análisis electroquímico es considerado una de las más poderosas técnicas dentro de este campo ${ }^{131}$, en particular los electrodos selectivos de iones (ESI) han tenido un desarrollo exponencial en las últimas décadas. Se han construido ESI para casi cualquier ion ${ }^{132}$, y su investigación ha sido constante.

Para $\mathrm{Hg}(\mathrm{II})$, han sido reportados numerosos ESI que utilizan distintos elementos de reconocimiento. Gracias a los sofisticados métodos de arquitectura molecular, ionóforos basados en: tioles ${ }^{133}$, bases de Schiff ${ }^{134,135}$, tioureas ${ }^{136,137}$, derivados de calixarenos ${ }^{138,139,140,141}$, fosfinas ${ }^{142}$ y ditiosulfatos ${ }^{143}$ han sido sintetizados para la determinación selectiva de iones mercurio.

Es sabido que el mercurio presenta una alta afinidad a grupos donadores de azufre, por lo tanto, paralelamente a los compuestos ya mencionados, se han diseñado ionóforos ${ }^{144,}{ }^{145} \mathrm{y}$ fluoróforos 146, 147, 148,149,150,151,152 que contienen este tipo de donadores de electrones en sus estructuras para así garantizar una alta selectividad hacia mercurio frente a posibles iones interferentes. Este capítulo se basa en el uso de acomplejantes del tipo O,O'-(2,2'bifenilen)ditiofosfato de Alquilo (alquilo= metilo, etilo, propilo o butilo) como materiales para la construcción de electrodos selectivos al ion $\mathrm{Hg}(\mathrm{II})$ de contacto interno sólido, se piensa que por la presencia de grupos donadores de azufre en su estructura, estos materiales tienen potencial 
aplicación en la preparación de membranas poliméricas para la determinación selectiva de iones mercurio.

\subsection{EXPERIMENTAL}

\subsubsection{MATERIALES Y MÉTODOS}

Los ionóforos: O,O'-(2,2'-bifenilen)ditiofosfato de metilo (MetDTF), O,O'-(2,2'bifenilen)ditiofosfato de etilo (EtDTF), O,O'-(2,2'-bifenilen)ditiofosfato de propilo (PropDTF) y O,O'-(2,2'-bifenilen)ditiofosfato de butilo (ButDTF) fueron sintetizados conforme al método descrito en el capítulo 2. Nitrato de mercurio(II) monohidratado se obtuvo de Sigma-Aldrich. Dibutilftalato (DBP), 2-nitrophenyloctyl ether (o-NPOE) y Bis(1-butylpentyl) Adipato (BBPA)fueron obtenidos de Fluka. Tetrafenil borato de sodio (NaTPB) y PVC de alto peso molecular se obtuvieron de Sigma-Aldrich. Araldith M y endurecedor HR se obtuvieron de CibaGeigy. Los nitratos correspondientes a las especies $\mathrm{Pb}(\mathrm{II}), \mathrm{Ni}(\mathrm{II}), \mathrm{NH}_{4}{ }^{+} \mathrm{Na}^{+}, \mathrm{K}^{+}, \mathrm{Cu}(\mathrm{II}), \mathrm{Cr}(\mathrm{III})$, $\mathrm{Co}(\mathrm{II})$ y $\mathrm{Al}(\mathrm{III})$ fueron obtenidas de Merck. Soluciones de diferentes concentraciones se prepararon por dilución a partir de una solución $0.1 \mathrm{~mol} \mathrm{~L}^{-1}$ utilizando agua desionizada (18 M $\Omega$ cm de resistividad) de un equipo Milli-Q de Millipore. 


\subsubsection{CONSTRUCCIÓN DEL ELECTRODO “COMPOSITE" DE CONTACTO INTERNO SÓLIDO}

Fueron mezclados $0.5 \mathrm{~g}$ de araldith $\mathrm{M}$ y $0.2 \mathrm{~g}$ de endurecedor HR con $0.7 \mathrm{~g}$ de polvo de grafito hasta tener una mezcla homogénea. Una pequeña porción de la mezcla es depositada en un tubo de PVC de $2.0 \mathrm{~cm}$ de largo y $7.0 \mathrm{~mm}$ de diámetro que dentro contiene un disco de cobre, el espesor de la matriz carbón-epoxy es aproximadamente de $3.0 \mathrm{~mm}$. El electrodo se dejó curar a $40{ }^{\circ} \mathrm{C}$ por 24 horas.

\subsubsection{PREPARACIÓN DE LAS MEMBRANAS SENSORAS}

Se pesaron las cantidades proporcionales de ionóforo/plastificante/PVC/ NaTPB en un vaso de precipitados de $5.0 \mathrm{~mL}$ y esta mezcla se disolvió en $1.0 \mathrm{~mL}$ de THF. Se tomaron $20 \mu \mathrm{L}$ de esta mezcla sensora, depositándolos sobre el electrodo composite y polimerizando a $40{ }^{\circ} \mathrm{C}$ por 1 hora.

\subsubsection{MEDICIÓN DEL POTENCIAL}

Las determinaciones de mercurio(II) se realizaron por cronopotenciometría a corriente nula en un potenciostato/galvanostato $\mu$ AutoLab Type III . Se utilizó un sistema de dos electrodos, como electrodo de trabajo los ESI aquí construidos y $\mathrm{Ag} / \mathrm{AgCl}$ como referencia externa. Todas las pruebas se realizaron en una celda electroquímica convencional con $10 \mathrm{~mL}$ de solución $\mathrm{HClO}_{4}$ $1.0 \mathrm{~mol} \mathrm{~L}^{-1} \mathrm{pH} 0$. 


\subsection{RESULTADOS Y DISCUSIÓN}

En la siguiente figura se muestra la posible forma de coordinación que los ionóforos podrían presentar frente al ion mercurio.

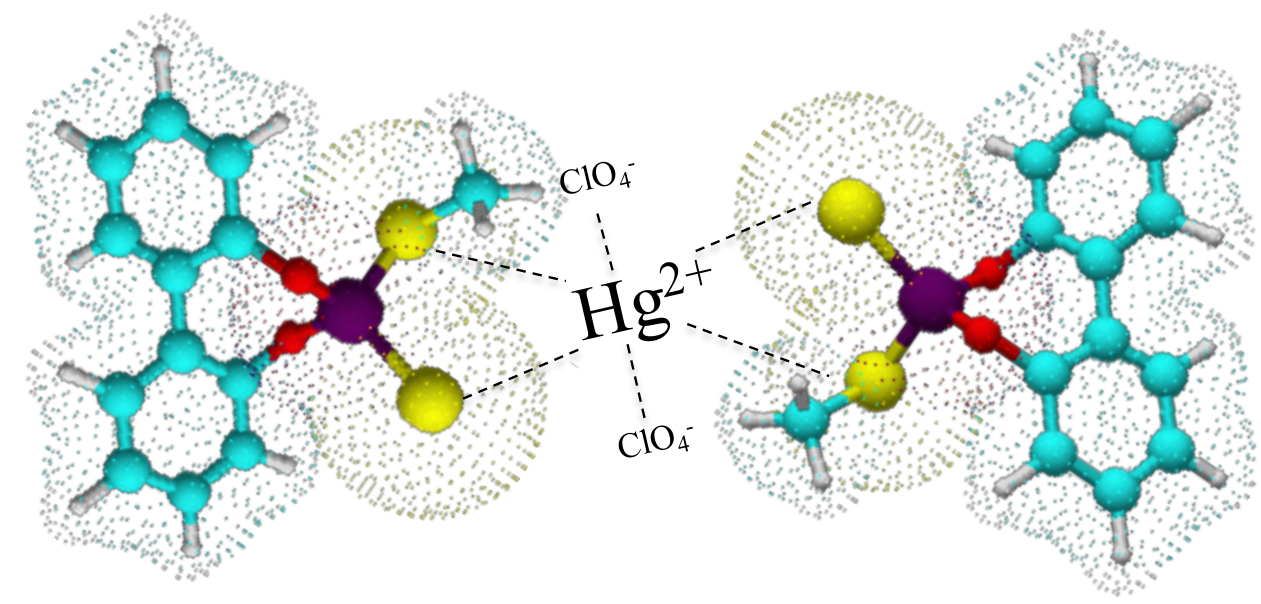

Figura 4.1. Posible estructura de coordinación de MetDTF con el ion mercurio.

\subsubsection{EFECTO DE LA CADENA ALQUÍliCA Y DEL ACONDICIONAMIENTO DE LA MEMBRANA}

Para los electrodos selectivos de iones su elemento de reconocimiento o ionóforo es el principal parámetro que hay que tomar en cuenta a la hora de su construcción, por ello se sintetizaron cuatro moléculas de la misma familia, todas ellas candidatas a ser el mejor elemento de reconocimiento para la construcción de un nuevo electrodo selectivo a iones $\mathrm{Hg}$ (II) de contacto interno sólido. La diferencia en las cuatro estructuras es el grupo alquilo enlazado a uno de los grupos donadores de azufre, la cadena alifática puede ser: metilo, etilo, propilo o butilo. 
Se prepararon electrodos para cada uno de los ionóforos sintetizados utilizando la composición siguiente, ionóforo:o-NPOE:PVC:NaTPB de 5:62:33:1.0mg (\%, w/w), las membranas se acondicionaron en una solución $1.0 \times 10^{-2} \mathrm{~mol} \mathrm{~L}^{-1}$ de $\mathrm{Hg}$ (II) durante 3 y 24 horas y la respuesta potenciométrica de los sensores se probó en un amplio intervalo de concentraciones de $\mathrm{Hg}$ (II) de $\left(1.0 \times 10^{-13}\right.$ a $\left.1.0 \times 10^{-5}\right) \mathrm{mol} \mathrm{L}^{-1}$ en $10 \mathrm{~mL}$ de solución $1.0 \mathrm{~mol} \mathrm{~L}^{-1}$ de $\mathrm{HClO}_{4}$. En la Figura $4.2 \mathrm{se}$ muestran las curvas de calibración potenciométricas para los cuatro electrodos construidos y acondicionados a diferentes tiempos.

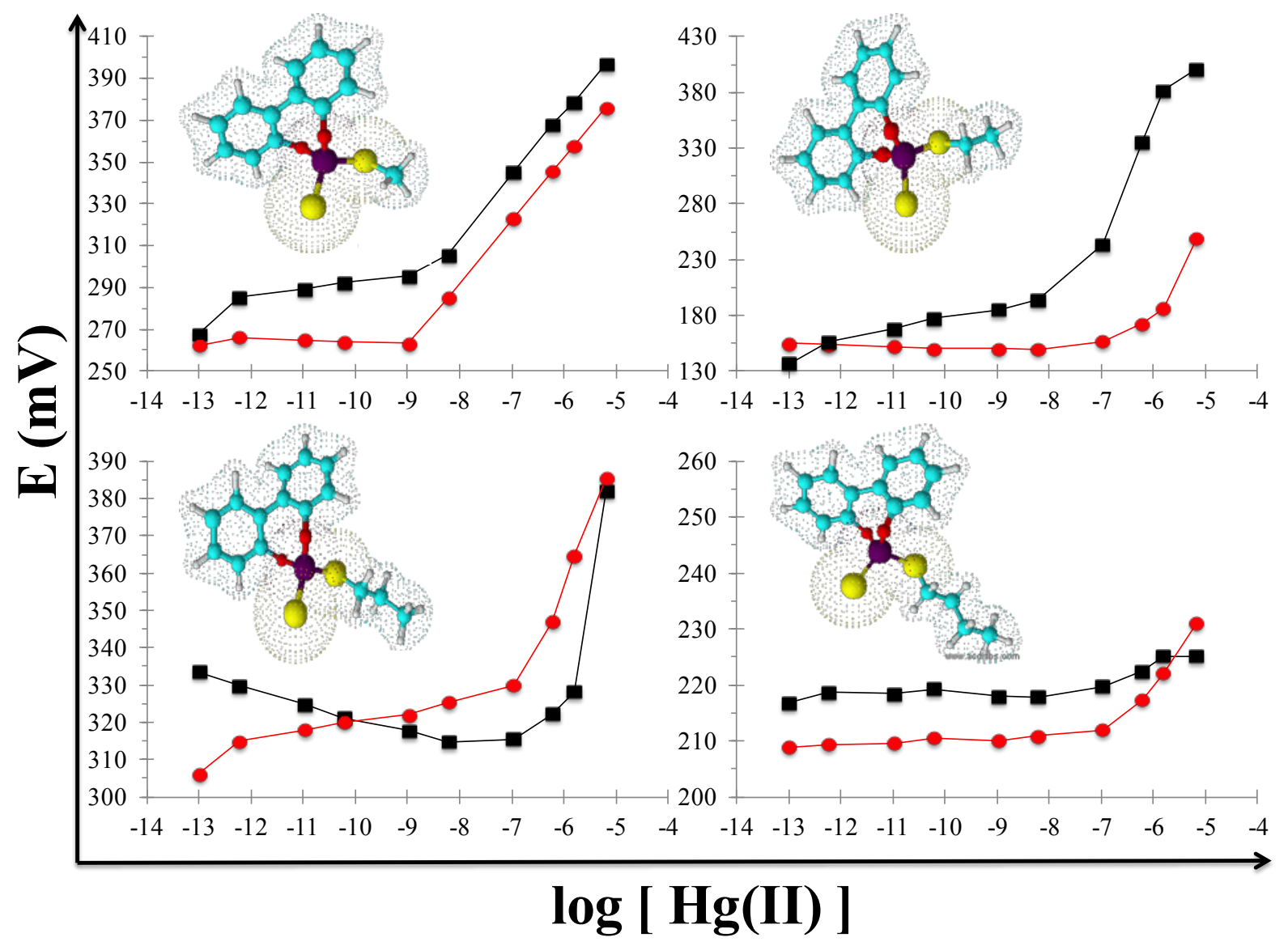

Figura 4.2. Curvas de calibración potenciométricas de los electrodos de contacto interno sólido selectivos al ion $\mathrm{Hg}$ (II) por la adición de alícuotas de $\mathrm{Hg}\left(\mathrm{NO}_{3}\right)_{2}$ en solución acuosa a pH 0 con $\mathrm{HClO}_{4} 1.0 \mathrm{~mol} \mathrm{~L}^{-1}\left(\boldsymbol{\square}, 3\right.$ horas de acondicionamiento; ${ }^{\bullet}, 24$ horas de acondicionamiento). 
Se observa que el efecto que tiene la cadena alifática sobre la respuesta potenciométrica de los electrodos es fuerte. Las cadenas alifáticas pueden ocasionar dos efectos en compuestos de coordinación, el primero es el efecto inductivo, que es la capacidad de donar carga por la polarización de electrones en los enlaces $\sigma$ y el segundo, el efecto estérico, que es la repulsión relacionada con el volumen de un sustituyente. El efecto inductivo tiene la propiedad de estabilizar complejos catiónicos, pero en el caso de las cuatro estructuras que se probaron no fue así, el efecto estérico predominó frente al efecto inductivo y la repulsión que presentaron los grupos etilo, propilo y butilo desestabilizaron la molécula. Por su parte, el metilo presenta el menor efecto de inducción, pero también el menor efecto estérico y al parecer dentro de la membrana polimérica es deseable no tener un fuerte efecto estérico para formación óptima del complejo con $\mathrm{Hg}(\mathrm{II})$, esto lo demuestran los resultados obtenidos para el electrodo construido con el ionóforo MetDTF, ya que presenta el mejor comportamiento en cuanto a estabilidad en la señal potenciométrica, tanto después de 3 horas de acondicionamiento como a 24 horas, aunque resulta ser que a 24 horas de acondicionado el electrodo, su respuesta potenciométrica es invariante con una pendiente Nernstiana de $(29.8 \pm 0.2) \mathrm{mVdecada}^{-1}$. Por todo lo ya mencionado, el ionóforo MetDTF se propone para todas las pruebas siguientes.

\subsubsection{EFECTO DEL PLASTIFICANTE}

Fueron probados tres plastificantes: DBP, $o$-NPOE y BBPA, para determinar cual de ellos es el mejor para la construcción de un nuevo electrodo de contacto sólido selectivo a iones $\mathrm{Hg}$ (II) con MetDTF como ionóforo. La composición de la membrana fue 5:62:33:1.0mg (\%, w/w) pero esta 
vez variando el plastificante. Cada electrodo construido fue acondicionado por 24 horas en solución $1.0 \times 10^{-2} \mathrm{~mol} \mathrm{\textrm {L } ^ { - 1 }}$ de iones $\mathrm{Hg}(\mathrm{II})$ y se construyeron curvas de calibración potenciométricas en un intervalo de concentraciones de $1.0 \times 10^{-13}$ a $1.0 \times 10^{-5} \mathrm{~mol} \mathrm{~L}^{-1}$ en $10 \mathrm{~mL}$ de solución $1.0 \mathrm{~mol} \mathrm{~L}^{-1}$ de $\mathrm{HClO}_{4}$. En la Figura 4.3 se muestra la variación del potencial de membrana en función de la concentración de iones mercurio.



Figura 4.3. Curvas de calibración potenciométricas por la adición de alícuotas de $\mathrm{Hg}\left(\mathrm{NO}_{3}\right)_{2}$ en solución acuosa a pH 0 con $\mathrm{HClO}_{4} 1.0 \mathrm{~mol} \mathrm{~L}^{-1}$ de los ESI construidos con distintos plastificantes $\left[\square, \mathrm{BBPA}\right.$ (pendiente $=187 \pm 2 \mathrm{mV}$ decada $\left.^{-1}\right) ; \bullet, o-\mathrm{NPOE}\left(\right.$ pendiente $=29.8 \pm 0.2 \mathrm{mV}$ decada ${ }^{-}$ $\left.{ }^{1}\right) ; \Delta, \mathrm{DBP}\left(\right.$ pendiente $=247 \pm 23 \mathrm{mV}$ decada $\left.\left.^{-1}\right)\right]$. 
Como se observa en la Figura 4.3, el BBPA y DBP presentan pendientes muy por encima por las predichas por la ecuación de Nernst esto puede deberse a que estos plastificantes permiten la entrada excesiva de iones $\operatorname{Hg}(\mathrm{II})$ a la matriz polimérica y aun que no todos estos iones están formando complejos con el MetDTF se encuentran dentro de la membrana y claramente participan en las mediciones del potencial en la interfaz membrana/solución. El mejor plastificante cuando se usa MetDTF como ionóforo resulta ser el $o$-NPOE presentado una respuesta Nernstiana de $(29.8 \pm 0.2) \mathrm{mV}$ década $^{-1}$ para un intervalo de concentraciones de $(1.1 \mathrm{x}$ $10^{-9}$ a $\left.1.0 \times 10^{-5}\right) \mathrm{mol} \mathrm{L}^{-1}$ y con un límite de detección de $(9.7 \pm 0.6) \times 10^{-10} \mathrm{~mol} \mathrm{~L}^{-1}$, por esto el $o$ NPOE se propone como plastificante para la construcción de ISE's en estudios posteriores.

\subsubsection{EFECTO DE LA CONCENTRACIÓN DEL IONÓFORO}

Para el estudio del efecto de la concentración del ionóforo MetDTF sobre la respuesta potenciométrica del ISE propuesto se estudiaron cinco membranas polimérica de distintas composiciones. La concentración del ionóforo fue variada en 1, 2, 3, 4 y $5 \%$. Cada membrana fue acondicionada por 24 horas en solución $1.0 \times 10^{-2} \mathrm{~mol} \mathrm{~L}^{-1}$ de iones $\mathrm{Hg}(\mathrm{II})$. A partir de la respuesta potenciométrica de las membranas propuestas se calcularon la pendiente y el límite de detección para cada sensor, estos parámetros se muestran en la Tabla 4.1. 
Tabla 4.1. Parámetros analíticos para los electrodos de contacto sólido selectivos a iones $\mathrm{Hg}$ (II) con membranas de diferente composición, probados en un intervalo de concentraciones de mercurio de $\left(1.0 \times 10^{-13}\right.$ a $\left.1.0 \times 10^{-5}\right) \mathrm{mol} \mathrm{L}-1$ a pH 0 impuesto con $10 \mathrm{~mL}$ de solución $1.0 \mathrm{~mol} \mathrm{~L}{ }^{-1}$ de $\mathrm{HClO}_{4}$.

\begin{tabular}{|c|c|c|c|c|c|c|c|}
\hline \multirow{2}{*}{$\begin{array}{c}\text { Hg(II)- } \\
\text { ISE- } \\
\text { MetDTF }^{\mathrm{a}}\end{array}$} & \multicolumn{4}{|c|}{ Composición $(\%$, w/w) } & \multirow{2}{*}{$\begin{array}{c}\text { Pendiente } \\
\left(\mathrm{mV} \text { decada }^{-1}\right)\end{array}$} & \multirow{2}{*}{$\begin{array}{c}\text { LDD } \\
\left(\mathrm{mol} \mathrm{L}^{-1}\right)\end{array}$} & \multirow[b]{2}{*}{$\mathbf{r}^{2}$} \\
\hline & MetDTF & $o-\mathrm{NPOE}$ & PVC & NaTPB (mg) & & & \\
\hline 1 & 5 & 62 & 33 & 0.0 & $54 \pm 14$ & $(6 \pm 1) \times 10^{-7}$ & 0.9169 \\
\hline 2 & 1 & 66 & 33 & 1.0 & $44 \pm 3$ & $(4.8 \pm 0.9) \times 10^{-8}$ & 0.9958 \\
\hline 3 & 2 & 65 & 33 & 1.0 & $107 \pm 21$ & $(5 \pm 2) \times 10^{-7}$ & 0.9612 \\
\hline 4 & 3 & 64 & 33 & 1.0 & $47 \pm 6$ & $(1.8 \pm 0.9) \times 10^{-8}$ & 0.9407 \\
\hline 5 & 4 & 63 & 33 & 1.0 & $39 \pm 4$ & $(9.2 \pm 0.8) \times 10^{-8}$ & 0.9915 \\
\hline 6 & 5 & 62 & 33 & 1.0 & $29.8 \pm 0.2$ & $(9.7 \pm 0.6) \times 10^{-10}$ & 0.9999 \\
\hline
\end{tabular}

${ }^{\text {a }} \mathrm{Hg}$ (II)-ISE-MetDTF (electrodo de contacto sólido selectivo a iones $\mathrm{Hg}$ (II) basado en MetDTF)

En la Tabla 4.1 se puede apreciar que la composición 1, la cual no contiene NaTPB, presenta una respuesta super-Nernstiana y un LDD alrededor de $10^{-7} \mathrm{~mol} \mathrm{~L}^{-1}$, mientras que las composiciones 2, 3 y 4 que corresponden a 1,2 y $3 \%$ de ionóforo presentan respuestas similares, solo que el LDD para las composiciones 2 y 4 están alrededor de $10^{-8} \mathrm{~mol} \mathrm{~L}^{-1}$. Los mejores parámetros analíticos se obtuvieron para las composiciones 5 y 6 que contienen 4 y $5 \%$ de ionóforo en la membrana sensora, para la composición 5 se obtuvo una pendiente que aún que super-Nernstiana se acerca más a la predicha por la teoría y con límites de detección alrededor de $10^{-8} \mathrm{~mol} \mathrm{~L} \mathrm{~L}^{-1}$. La Tabla 1.1 también muestra que el aumento en la concentración del ionóforo mejora el rendimiento de los sensores en términos de la pendiente y límite de detección. Además se observa que la mejor composición la tiene el sensor $\mathrm{Hg}$ (II)-ISE-MetDTF-6 ya que presenta 
una respuesta Nernstiana de $(29.8 \pm 0.2) \mathrm{mV} \mathrm{decada}^{-1}$ con un LDD de $(9.7 \pm 0.6) \times 10^{-10} \mathrm{~mol} \mathrm{~L}^{-1} \mathrm{y}$ por lo tanto esta composición se eligió para todos los estudios adicionales.

\subsubsection{REPRODUCIBILIDAD}

Se realizó el estudio de reproducibilidad para el sensor Hg(II)-ISE-MetDTF-6 haciendo dos curvas de calibración por cinco días consecutivo en un amplio intervalo de concentraciones de $\mathrm{Hg}(\mathrm{II})\left(1.0 \times 10^{-13}\right.$ a $\left.1.0 \times 10^{-1}\right) \mathrm{mol} \mathrm{L}^{-1}$, alícuotas del ion fueron adicionadas a $10 \mathrm{~mL}$ de solución pH 0 impuesto con $\mathrm{HClO}_{4} 1.0 \mathrm{~mol} \mathrm{~L}$. La Figura 4.4 muestra el promedio de estas curvas $(\mathrm{n}=10)$. Se obtuvo una pendiente promedio de $(29.8 \pm 0.4) \mathrm{mV}$ decada $^{-1}$ en un intervalo de concentraciones de $\left(1.0 \times 10^{-9}\right.$ a $\left.1.0 \times 10^{-1}\right) \mathrm{mol} \mathrm{L}^{-1}$ y con un límite de detección promedio de $(9.1$ $\pm 0.8) \times 10^{-10} \mathrm{~mol} \mathrm{~L}^{-1}$. Los resultados obtenidos demuestran que la respuesta el sensor $\mathrm{Hg}(\mathrm{II})$-ISEMetDTF-6 es altamente reproducible cuando se construye, acondiciona y prueba con la metodología descrita. 


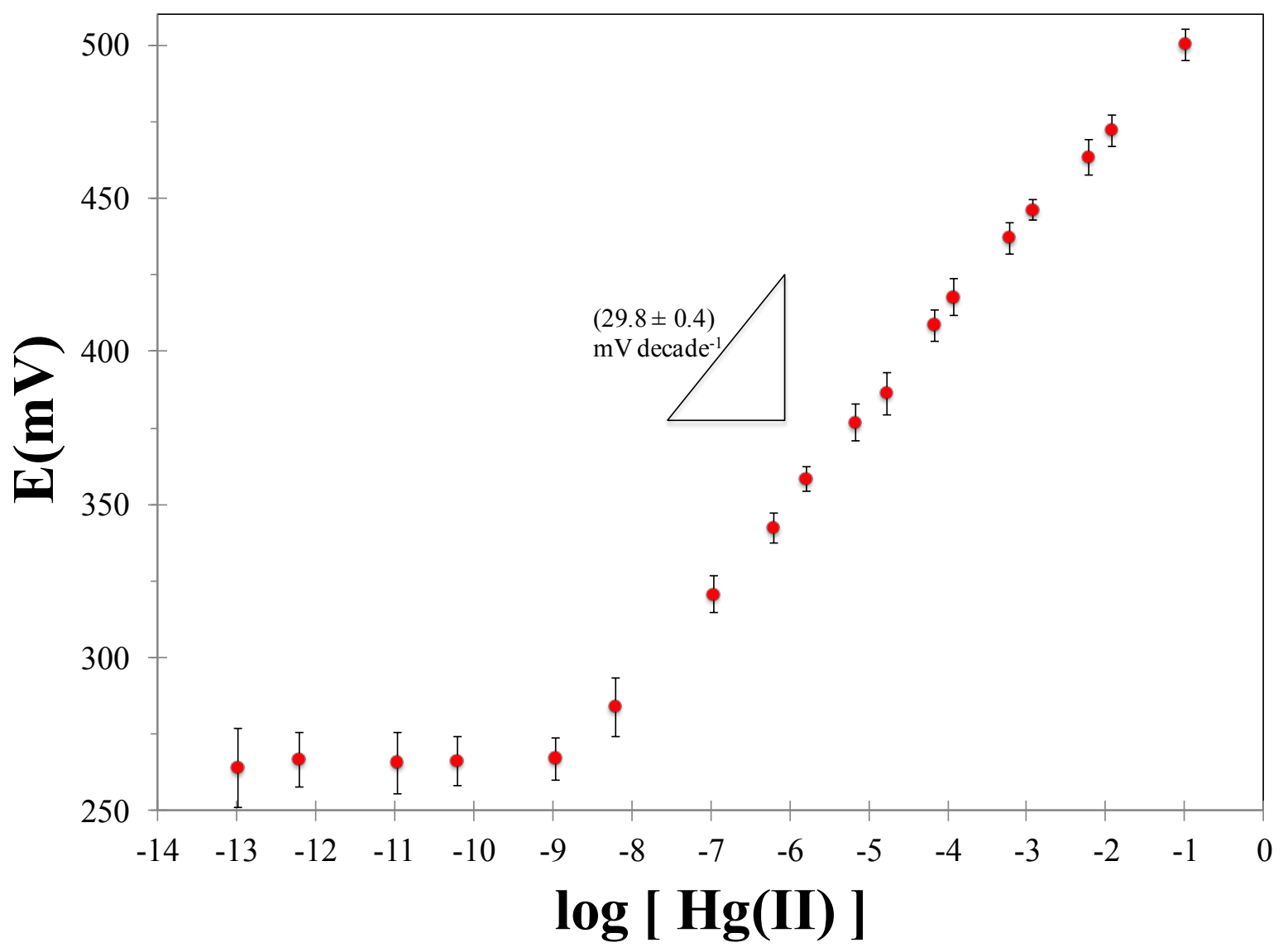

Figura 4.4. Promedio ( $\mathrm{n}=10)$ de las curvas de calibración potenciométricas del sensor $\mathrm{Hg}(\mathrm{II})-$ ISE-MetDTF-6 por la adición de alícuotas de $\mathrm{Hg}\left(\mathrm{NO}_{3}\right)_{2}$ en solución acuosa a pH 0 con $\mathrm{HClO}_{4}$ $1.0 \mathrm{~mol} \mathrm{~L}^{-1}$ [pendiente $=(29.8 \pm 0.4) \mathrm{mV}$ decada $^{-1}, \mathrm{LDD}<1 \mathrm{nmol} \mathrm{L}^{-1}$ ].

\subsubsection{EFECTO DEL $\mathrm{pH}$}

La influencia del $\mathrm{pH}$ sobre el potencial de membrana fue probado manteniendo constante la concentración de iones mercurio en $1.0 \times 10^{-3} \mathrm{~mol} \mathrm{~L}^{-1}$ y variando el $\mathrm{pH}$ en un intervalo de 0 - 7 , se prepararon soluciones $1.0 \mathrm{~mol} \mathrm{~L}^{-1}$ de $\mathrm{HClO}_{4}$ y se ajustaron al $\mathrm{pH}$ deseado con $\mathrm{NaOH} 1.0 \mathrm{~mol} \mathrm{~L}^{-1}$. 
${ }^{153}$ Los resultados son presentados en la Figura 4.5, donde el potencial del sensor $\mathrm{Hg}(\mathrm{II})$-ISEMetDTF-6 se mantuvo constante desde un pH 0 y hasta pH 6. Después de pH 6, se observa una drástica disminución en el potencial debido a la presencia de especies insolubles de mercurio, la formación de especies insolubles se demuestra en trabajos previos con la construcción del diagrama de existencia predominio (DEP) para $\mathrm{Hg}(\mathrm{II}) .{ }^{153}$ También se observa en la Figura 4.5 que sensor propuesto no presenta variación a pH's bajos, esto demuestra que no existe interacción entre el ionóforo MetDTF y los iones $\mathrm{H}^{+}$. Por lo tanto el intervalo de $\mathrm{pH}$ de 0 a 6 fue tomado como intervalo de trabajo para el sensor $\mathrm{Hg}$ (II)-ISE-MetDTF-6.

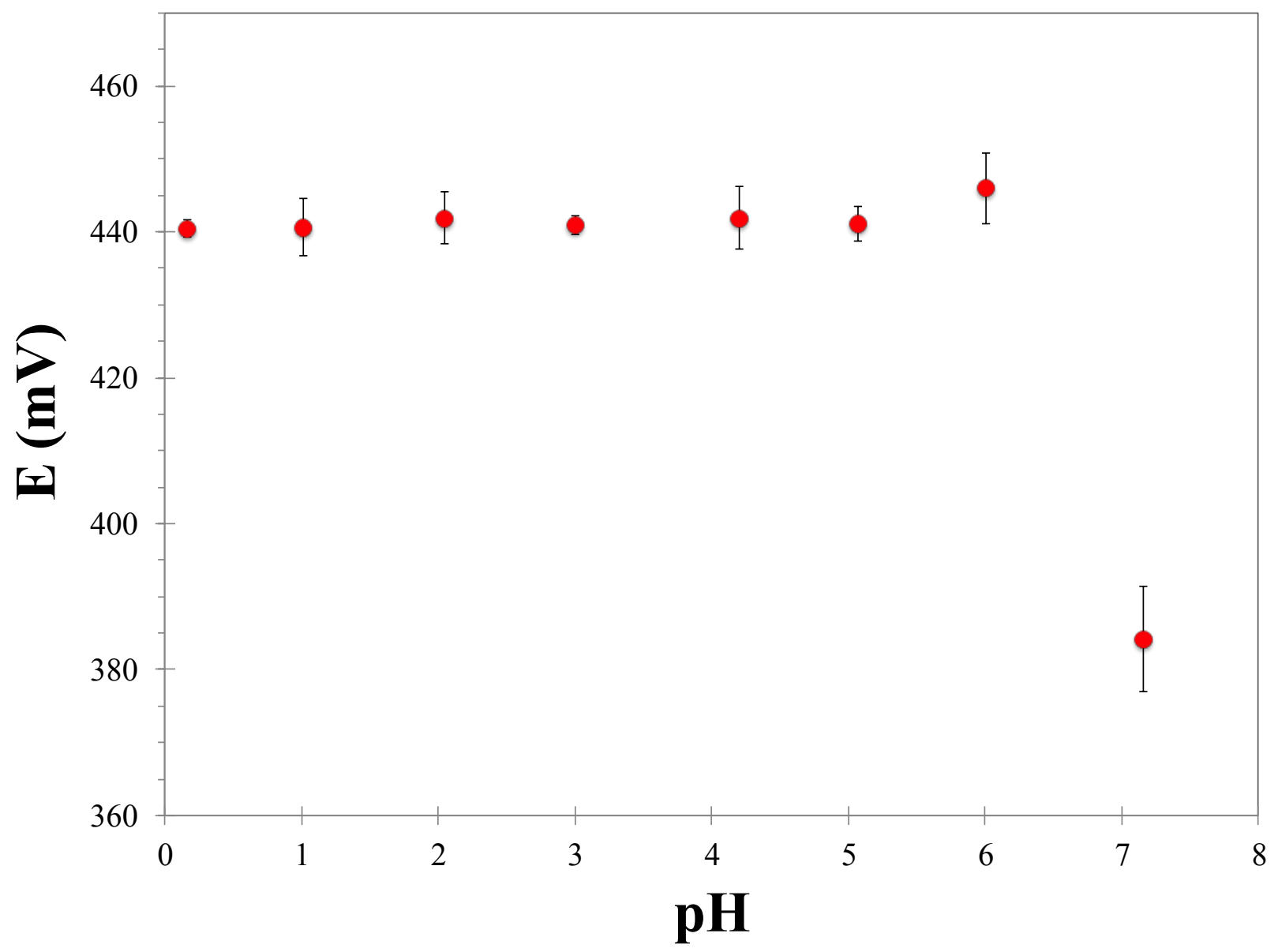

Figura 4.5. Influencia del pH sobre la respuesta del sensor Hg(II)-ISE-MetDTF-6en solución de $\mathrm{Hg}$ (II) $1.0 \times 10^{-3} \mathrm{~mol} \mathrm{~L}^{-1}$ y ajustando el $\mathrm{pH}$ con $\mathrm{HClO}_{4}$ y $\mathrm{NaOH} 1.0 \mathrm{~mol} \mathrm{~L}$. 


\subsubsection{SELECTIVIDAD}

La característica más importante de un ISE es la capacidad de responder selectivamente a un ion primario en presencia de otros iones, este parámetro está directamente relacionado con el equilibrio termodinámico en la interfase membrana/solución. La selectividad del sensor $\operatorname{Hg}(\mathrm{II})$ ISE-MetDTF-6 fue probado para distintas especies catiónicas. Para cationes divalentes se evalúan las constantes potenciométricas utilizando el fixed interferece method (FIM) y para las especies catiónicas de cargas distintas se reportan sus curvas de calibración.

\subsubsection{SELECTIVIDAD HACIA CATIONES DIVALENTES}

El coeficiente de selectividad potenciométrico (que se mide en términos de la constante de selectividad potenciométrica $\left(K_{H g(I I), j}^{P o t}\right)$ describe la preferencia del sensor $\mathrm{Hg}(\mathrm{II})-\mathrm{ISE}-\mathrm{MetDTF}-6$ a un ión interferente en presencia de $\mathrm{Hg}(\mathrm{II})$, las constantes fueron determinadas mediante el fixed interferece method (FIM) el cual se basa en la ecuación semiempírica de Nikolsky-Eisenman ${ }^{154,}$ 155.

$$
E_{I S E}=E^{0}+\frac{R T}{Z_{i} F} \ln \left[a_{i}+\sum_{j} K_{i, j}^{p o t} a_{j}^{z_{i} / z_{j}}\right]
$$

Donde $\mathrm{E}_{\mathrm{ISE}}$ es el potencial medido, $\mathrm{E}^{\mathrm{o}}$ el potencial estándar del sensor, $\mathrm{a}_{\mathrm{i}} \mathrm{y} \mathrm{a}_{\mathrm{j}}$ las actividades de los iones $\mathrm{Hg}(\mathrm{II})$ e interferente respectivamente, $z_{i}$ la carga del ion primario $\mathrm{y}, \mathrm{R}, \mathrm{T}$ y $\mathrm{F}$ tienen el significado habitual. En el método FIM, la concentración de mercurio es variada en $10 \mathrm{~mL}$ de 
una solución $1.0 \mathrm{~mol} \mathrm{~L}^{-1}$ de $\mathrm{HClO}_{4}$ y la concentración del ion interferente se mantiene constante en $1.0 \times 10^{-2} \mathrm{~mol} \mathrm{~L}^{-1}$. En la Tabla 4.2 se muestran los valores de las constantes potenciométricas para los cationes divalentes probados. Un valor igual a 1.0 indica que el sensor responde de manera similar para el ion primario e interferente. Entonces entre más pequeño sea el valor de la constante potenciométrica más alta será la selectividad del sensor. Se puede observar en la Tabla 4.2 que los valores de las constantes potenciométricas calculadas son mucho menores a 1.0, demostrando con esto que la selectividad del sensor $\mathrm{Hg}$ (II)-ISE-MetDTF-6 hacia iones $\mathrm{Hg}$ (II) es alta frente a los cationes divalentes estudiados.

Tabla 4.2. Coeficientes de selectividad potenciométricos $\left(-\log K_{H g(I I), j}^{P o t}\right)$ para el sensor $\operatorname{Hg}($ II)ISE-MetDTF-6 evaluados con en método FIM y manteniendo constante la concentración de $\mathrm{Hg}(\mathrm{II})$ en $1.0 \times 10^{-2} \mathrm{~mol} \mathrm{~L}{ }^{-1}$.

\begin{tabular}{cc}
\hline $\begin{array}{c}\text { Ion } \\
\text { Interferente }\end{array}$ & $-\log K_{H g(I I), j}^{\text {Pot }}$ \\
\hline $\mathrm{Pb}(\mathrm{II})$ & $6.9 \pm 0.8$ \\
$\mathrm{Ni}(\mathrm{II})$ & $6.1 \pm 0.8$ \\
$\mathrm{Mg}(\mathrm{II})$ & $7.4 \pm 0.9$ \\
$\mathrm{Cu}(\mathrm{II})$ & $5.3 \pm 0.7$ \\
$\mathrm{Co}(\mathrm{II})$ & $7.2 \pm 0.9$ \\
\hline
\end{tabular}


Ya que se ha reportado que con el método FIM no es posible calcular constantes de selectividad confiables cuando se evalúan iones con distinto estado de oxidación que el ion principal ${ }^{156,157}$, se evaluó la respuesta potenciométrica del sensor hacia estos iones. Las curvas de calibración se construyeron en las mismas condiciones que las pruebas anteriores solo que se adicionaron alícuotas del ion interferente. En la Figura 4.6 se muestran los resultados obtenidos para distintos iones interferentes, se puede observar que para la mayoría de los iones investigados el sensor $\mathrm{Hg}(\mathrm{II})$-ISE-MetDTF-6 no presenta respuesta potenciométrica significativa. También se ve en la figura que el sensor presenta respuesta para el ion Fe(III) desde concentraciones alrededor de $10^{-7} \mathrm{~mol} \mathrm{~L}{ }^{-1}$, pero con una pendiente de $(5.1 \pm 0.9) \mathrm{mV}$ decada $^{-1}$ que está muy por debajo de la predicha por la ecuación de Nernst para cationes trivalentes por tanto se puede decir que la respuesta del sensor hacia Fe(III) es despreciable. 


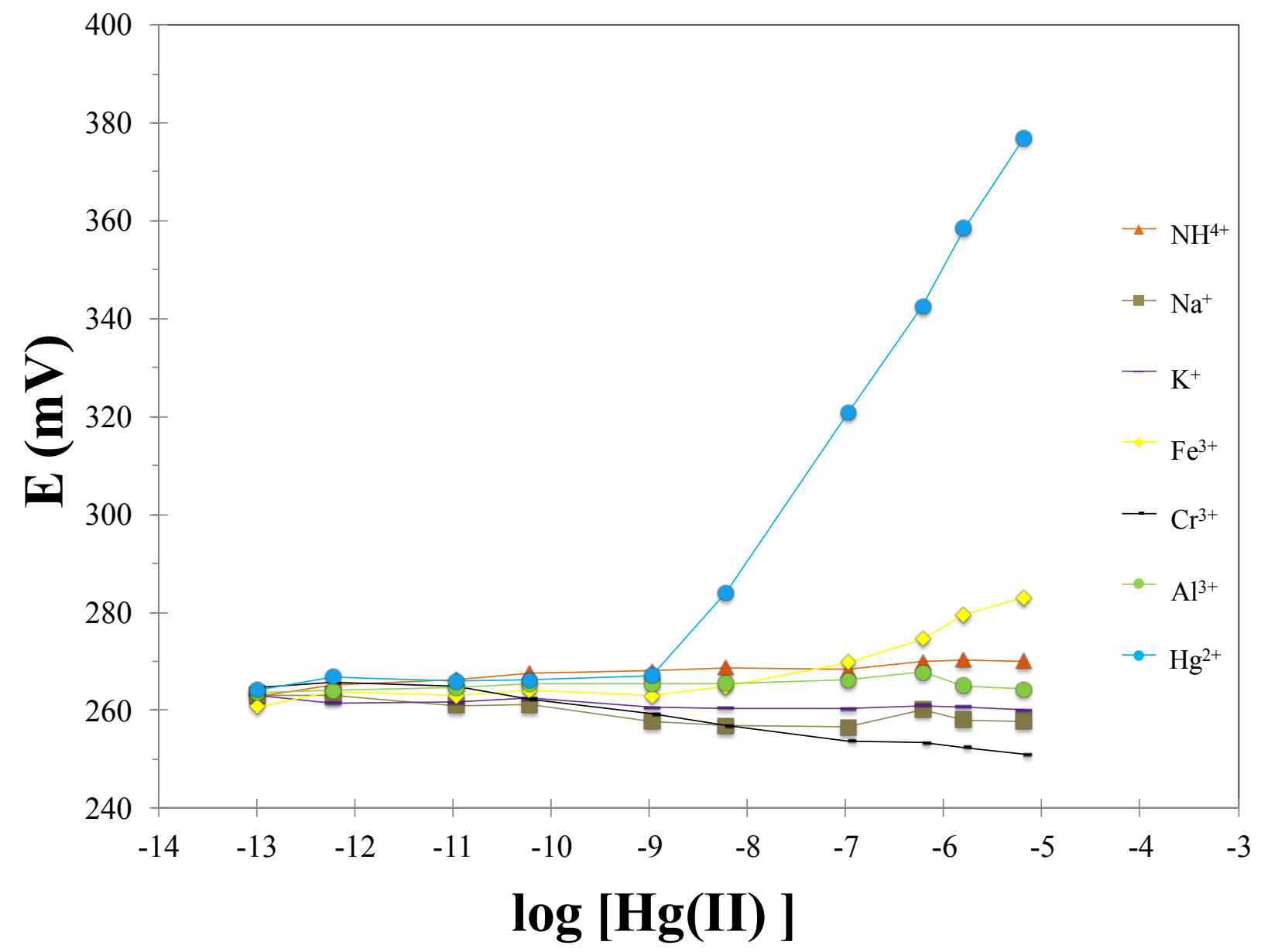

Figura 4.6. Respuesta potenciométrica del sensor Hg(II)-ISE-MetDTF-6 por la adicción de alícuotas de distintos iones interferentes de distinta carga a $\mathrm{Hg}(\mathrm{II})$ en solución acuosa $\mathrm{pH} 0$ con $\mathrm{HClO}_{4} 1.0 \mathrm{~mol} \mathrm{~L}^{-1}$.

\subsubsection{APLICACIÓN ANALÍTICA}

\subsubsection{DETERMINACIÓN DE MERCURIO EN MUESTRAS SINTÉTICAS}

El sensor $\operatorname{Hg}$ (II)-ISE-MetDTF-6 se utilizó para la determinación de iones mercurio en muestras sintéticas de concentraciones $1.0 \times 10^{-3}, 1.0 \times 10^{-4}$ y $1.0 \times 10^{-5} \mathrm{~mol} \mathrm{~L}^{-1}$ a pH 0 con $\mathrm{HClO}_{4}$ 
$1.0 \mathrm{~mol} \mathrm{~L}^{-1}$. En la Tabla 4.3 se muestran los porcentajes de recobro para las diferentes concentraciones examinadas, se nota que los errores de dichos recobros son menores al 5\%, entonces podemos decir que el sensor propuesto puede determinar la concentración de mercurio en muestras acuosas.

Tabla 4.3. Porcentajes de recobro para diferentes muestras sintéticas de mercurio $(n=3)$.

\begin{tabular}{lccc}
\hline $\left.\begin{array}{l}{[\mathbf{H g}(\mathrm{II})]} \\
(\mathbf{m o l ~ L}\end{array}\right)$ & \begin{tabular}{c}
$\mathbf{E}_{\text {Hg(II)-ISE-MetDTF-6 }}(\mathbf{m V})$ \\
\hline $1.0 \times 10^{-3}$
\end{tabular} & $\begin{array}{c}{[\mathbf{H g}(\mathrm{II})]} \\
\text { recuperada } \\
\left(\mathbf{m o l ~ L}^{-1}\right)\end{array}$ & $\begin{array}{c}\text { \% de } \\
\text { recobro }\end{array}$ \\
$1.0 \times 10^{-4}$ & 441 & $9.7 \times 10^{-4}$ & $97.1 \pm 4.1$ \\
$1.0 \times 10^{-5}$ & 312 & $1.0 \times 10^{-4}$ & $101.7 \pm 3.2$ \\
\hline
\end{tabular}

\subsubsection{VALORACIÓN POTENCIOMÉTRICA}

El sensor $\mathrm{Hg}(\mathrm{II})-\mathrm{ISE}-M e t D T F-6$ fue utilizado como electrodo indicador en la valoración potenciométrica de $\mathrm{Hg}(\mathrm{II})$ con EDTA. Las condiciones de la valoración potenciométrica fueron las reportadas previamente por nuestro grupo de investigación ${ }^{158}$. Se valoraron $10 \mathrm{ml}$ de una solución de $\mathrm{Hg}\left(\mathrm{NO}_{3}\right)_{2} 1.0 \times 10^{-3} \mathrm{~mol} \mathrm{~L}^{-1}$ con EDTA $1.0 \times 10^{-2} \mathrm{~mol} \mathrm{~L}-1$ a pH 5.0 impuesto con amortiguador de $\mathrm{HAc} / \mathrm{Ac}^{-} 1.0 \times 10^{-3} \mathrm{~mol} \mathrm{~L}^{-1}$. Los resultados de la titulación de $\mathrm{Hg}$ (II) se muestran en la Figura 4.7. El valorante añadido causó una disminución en el potencial como resultado de una disminución en la concentración del ion $\mathrm{Hg}(\mathrm{II})$ debido a la formación de un complejo con EDTA. La cantidad de iones $\mathrm{Hg}$ (II) en solución puede ser determinada con precisión a partir de las curvas de valoración resultantes, de la curva se observa que el volumen en el punto de equivalencia es $(1.0 \pm 0.03) \mathrm{mL}$ con lo cual se obtiene una concentración de $\mathrm{Hg}(\mathrm{II})$ de $\left(1.0 \times 10^{-3}\right.$ 
$\left.\pm 3.0 \times 10^{-5}\right) \mathrm{mol} \mathrm{L}^{-1}$, lo que demuestra que este sensor puede ser utilizado para la determinación de mercurio por métodos volumétricos. En la Figura 4.7 también se muestra la curva de valoración para mercurio en las mismas condiciones utilizando un electrodo de "J" de mercurio comercial, por lo tanto el sensor aquí propuesto es comparable con la repuesta de sensores comerciales.



Figura 4.7. Curvas de valoración potenciométricas de $10 \mathrm{ml} \mathrm{de} \mathrm{Hg}\left(\mathrm{NO}_{3}\right)_{2} 1.0 \times 10^{-3} \mathrm{~mol} \mathrm{~L}^{-1}$ con EDTA $1.0 \times 10^{-2} \mathrm{~mol} \mathrm{~L}^{-1}$ a pH 5.0 impuesto con amortiguador de HAc/Ac $1.0 \times 10^{-3} \mathrm{~mol} \mathrm{~L}^{-1}(\bullet$, utilizando el sensor $\mathrm{Hg}$ (II)-ISE-MetDTF-6; $\square$, utilizando un electrodo de "J" de mercurio comercial). En perspectiva, la primera derivada de las curvas de valoración. 


\subsubsection{COMPARACIÓN CON LA LITERATURA}

En la Tabla 4.4, se muestra una comparación de distintas metodologías reportadas en recientes años para la determinación de iones mercurio. Los métodos comprenden la aplicación de ISE, sensores fluorométricos, sensores voltamperométricos y hasta espectroscopía de absorción atómica. En la Tabla se puede observar que todos los métodos alcanzan límites de detección bajos siendo el más bajo el reportado en este trabajo. Aquí también se reporta el rango de pH más amplio y muy buena selectividad hacia mercurio. Por lo que podemos decir que el sensor propuesto es comparable en todos los sentidos con otros sensores y metodologías para determinar $\mathrm{Hg}(\mathrm{II})$. Adicionalmente, el sensor propuesto presenta ventajas frente a otros ya que es fácil de construir y almacenar, además de no ser costoso.

\subsection{CONCLUSIONES}

El electrodo $\mathrm{Hg}(\mathrm{II})-\mathrm{ISE}-\mathrm{MetDTF}-6$ mostró una respuesta Nernstiana para el $\mathrm{Hg}(\mathrm{II})$ de $29.8 \mathrm{mV}$ década $^{-1}$ (incluso en presencia de iones interferentes) dentro de un amplio intervalo de concentraciones . El intervalo de $\mathrm{pH}$ aplicable $(0.0$ - 6.0), el bajo límite de detección $(<1.0 \mathrm{nmol}$

$\mathrm{L}^{-1}$ ), y los coeficientes de selectividad potenciométricos, sugieren que el sensor propuesto es comparable con otros métodos utilizados para la determinación selectiva de este ión, dando ventajas adicionales con respecto a otros sensores propuestos en la literatura. El sensor basado en MetDTF exhibe diferentes ventajas, como en el costo de construcción, en el diseño de contacto sólido ya que es eliminada la referencia interna y la solución de referencia, lo que favorece su almacenamiento. Adicionalmente, el sensor propuesto se puede utilizar como electrodo indicador 
en la determinación de iones de $\mathrm{Hg}(\mathrm{II})$, ya que su respuesta es comparable con electrodos comerciales que utilizan el mismo principio.

Tabla 4.4. Métodos propuestos recientemente para la determinación selectiva de mercurio.

\begin{tabular}{|c|c|c|c|c|c|}
\hline Ref. & $\begin{array}{l}\text { Método o tipo } \\
\text { de sensor }\end{array}$ & $\begin{array}{c}\text { Pendiente } \\
\left.\text { (mVdécada }^{-1}\right)\end{array}$ & $\begin{array}{c}\text { LDD } \\
\left(\mathrm{mol} \mathrm{L}^{-1}\right)\end{array}$ & $\begin{array}{l}\text { pH de } \\
\text { trabajo }\end{array}$ & Selectividad, $-\log K_{H g(I I), j}^{P o t}$ \\
\hline 159 & FI-CVAAS $^{\alpha}$ & ----- & $1.2 \times 10^{-8}$ & ----- & ----- \\
\hline 160 & ISE & 29.5 & $1.0 \times 10^{-8}$ & $6.0-7.5$ & $\begin{array}{c}\mathrm{Mg}(\mathrm{II}), 4.7 ; \mathrm{Ba}(\mathrm{II}), 4.2 ; \mathrm{Sr}(\mathrm{II}) \\
\text { 4.2; } \mathrm{Ni}(\mathrm{II}), 3.5 ; \mathrm{Cu}(\mathrm{II}), 3.0 \\
\quad \mathrm{Zn}(\mathrm{II}), 4.3 ; \mathrm{Ca}(\mathrm{II}), 3.3\end{array}$ \\
\hline 161 & ISE & 29.4 & $7.2 \times 10^{-7}$ & $0.9-4.5$ & ----- \\
\hline 162 & $\mathrm{FS}^{\beta}$ & ----- & $1.0 \times 10^{-7}$ & 7.0 & ----- \\
\hline 163 & FS & ---- & $1.0 \times 10^{-7}$ & ----- & ----- \\
\hline 164 & $\mathrm{VS}^{\gamma}$ & ----- & $5.0 \times 10^{-9}$ & 4.0 & ----- \\
\hline 165 & $\begin{array}{l}\text { Biosensor de } \\
\text { ADN }\end{array}$ & ----- & $5.0 \times 10^{-9}$ & 7.4 & ----- \\
\hline $\begin{array}{l}\text { En este } \\
\text { trabajo }\end{array}$ & ISE & 29.8 & $9.1 \times 10^{-10}$ & $0.0-6.0$ & $\begin{array}{c}\mathrm{Pb}(\mathrm{II}), 6.9 ; \mathrm{Ni}(\mathrm{II}), 6.1 ; \mathrm{Mg}(\mathrm{II}), \\
\quad 7.4 ; \mathrm{Cu}(\mathrm{II}), 5.3 ; \mathrm{Co}(\mathrm{II}), 7.2\end{array}$ \\
\hline
\end{tabular}




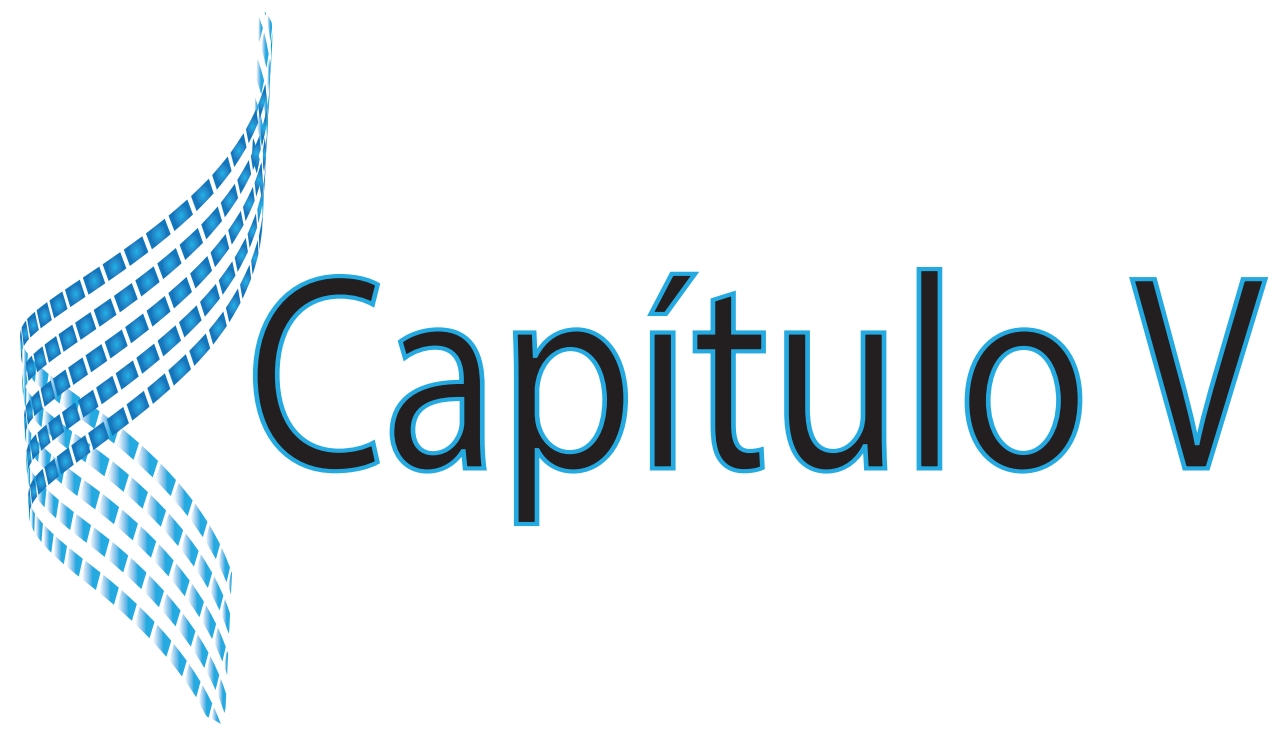

Nunca consideres el estudio como una obligación, sino como una oportunidad para penetrar en el bello y maravilloso mundo del saber.

Albert Einstein 


\section{CAPÍTULO V}

\section{CAPÍTULO V. DISEÑO DE CONTACTO INTERNO SÓLIDO BASADO EN UNA MATRIZ CARBÓN-EPOXY PARA LA CONSTRUCCIÓN DE ESI QUE USA UN IONÓFORO COMERCIAL}

\subsection{INTRODUCCIÓN}

El análisis potenciométrico basado en electrodos selectivos de iones (ISE), es un método simple que ha sido ampliamente empleado debido a que los dispositivos utilizados como electrodos de trabajo presentan innumerables ventajas tales como respuesta rápida, buena selectividad, un amplio intervalo de respuesta con bajos límites de detección, además de que su fabricación es sencilla, económica y su composición puede ser modificada fácilmente ${ }^{166,167}$. La composición de los ISE ha sido modificada desde los compuestos utilizados como agentes de reconocimiento (ionóforos) para la identificación de diferentes iones tanto orgánicos como inorgánicos $^{168}$, hasta la configuración del elemento de transducción. Clásicamente un ISE 
consiste en un electrodo de referencia interno (típicamente $\mathrm{Ag} / \mathrm{AgCl}$ o calomel), una solución interna y una membrana sensora, pero este diseño presenta algunas desventajas tanto en la construcción y almacenamiento, ya que la membrana no es fácil de colocar y la solución interna suele perderse. A estos inconvenientes un nuevo diseño de contacto interno sólido ha tomado fuerza en los últimos años. Muchos materiales se han utilizado como contacto sólido, por ejemplo, las sales orgánicas conductoras (SOC) tales como tetratiofulvalenotetracianoquinodimetano (TTF-TCNQ) las cuales poseen interesantes propiedades electroquímicas permitiendo una transferencia electrónica directa con compuestos electroactivos o bien actuando como mediadores en la construcción de nuevos electrodos ${ }^{169}$, pasta de carbón modificada con el ionóforo y plastificantes unida a un disco de cobre para la trasferencia de electrones ${ }^{170,171}$, también se han utilizado barras de grafito con contactos de cobre y plata ${ }^{172}$, carbón vítreo impregnado de plastificante y ionóforo ${ }^{173}$ y polímeros conductores como $\mathrm{MEH}$ PPV que utiliza un disco de oro como sustrato ${ }^{174}$.

Los materiales a base de grafito han sido muy utilizados en la fabricación de varios sensores electrométricos para diferentes propósitos analíticos ${ }^{175,176}$, ya que presenta valiosas propiedades fisicoquímicas como su conductividad y resistencia térmica, además de no ser costoso en comparación con metales nobles utilizados como conductores eléctricos. En este capítulo se utilizan las propiedades del grafito para la construcción de un ISE de contacto interno sólido basado en una matriz de carbón-epoxy utilizando una membrana sensora que incluye al ionóforo 1, 10-Dibenzyl-1, 10-diaza-18-crown-6 reportado como selectivo a iones $\mathrm{Hg}(\mathrm{II}){ }^{177}$, aunque este ionóforo también se ha reportado selectivo a iones $\mathrm{Ni}(\mathrm{II}){ }^{178}, \mathrm{~Pb}(\mathrm{II}){ }^{179} \mathrm{y} \mathrm{Li}^{+}{ }^{180}$. Dentro de los objetivos de este capítulo están el reportar un método simple, rápido y económico para las construcción de electrodos selectivos de iones, además de eliminar parámetros como el electrodo 
de referencia interna y el contacto líquido. Al prescindir de la solución interna se elimina el conductor iónico que tiene contacto con la referencia interna y juntos hacen el trabajo de un transductor electrónico pero pasando por dos procesos, primero en la membrana sensora es generada una diferencia de potencial debido a la diferencia de concentraciones, ésta diferencia de potencial induce una corriente eléctrica la cual es transportada por el conductor iónico hacia el electrodo de referencia interno que está constituido por un conductor electrónico, el cual enviará la señal generada hacia el detector. Entonces se propone la sustitución del transductor clásico por uno puramente electrónico como el grafito que facilitará el proceso de transducción para los ISE.

\subsection{EXPERIMENTAL}

\subsubsection{REACTIVOS Y SOLUCIONES}

Ionóforo selectivo a $\mathrm{Hg}$ (II) 1, 10-Dibenzyl-1, 10-diaza-18-crown-6 se obtuvo de Fluka. Nitrato de mercurio(II) monohidratado se obtuvo de Sigma-Aldrich. Dibutil butil fosfonato (DBBP) se obtuvo de Fluka. Tetrafenil borato de sodio (NaTPB) y PVC se obtuvieron de Sigma-Aldrich. Los nitratos correspondientes a las especies $\mathrm{Pb}(\mathrm{II}), \mathrm{Ni}(\mathrm{II}), \mathrm{NH}_{4}^{+} \mathrm{Na}^{+}, \mathrm{K}^{+}, \mathrm{Cu}(\mathrm{II}), \mathrm{Cr}(\mathrm{III}), \mathrm{Co}(\mathrm{II}) \mathrm{y}$ Al(III) fueron obtenidos de Merck. Soluciones de diferentes concentraciones se prepararon por dilución a partir de una solución $0.1 \mathrm{~mol} \mathrm{~L}^{-1}$ utilizando agua desionizada (18 $\mathrm{M} \Omega$ de resistividad) de un equipo Milli-Q de Millipore. 


\subsubsection{DISEÑO DEL ELECTRODO Y MEDICIÓN DE POTENCIAL}

Fueron mezclados $0.5 \mathrm{~g}$ de araldith M y $0.2 \mathrm{~g}$ de endurecedor HR (Ciba-Geigy) con $0.7 \mathrm{~g}$ de polvo de grafito hasta tener una mezcla homogénea. Una pequeña porción de la mezcla es depositada en un tubo de PVC de $2.0 \mathrm{~cm}$ de largo y $7.0 \mathrm{~mm}$ de diámetro que dentro contiene un disco de cobre, el espesor de la matriz carbón-epoxi es aproximadamente de $3.0 \mathrm{~mm}$. El electrodo se dejó curar a $40{ }^{\circ} \mathrm{C}$ por 24 horas. Sobre la superficie del electrodo son depositados 20 $\mu \mathrm{L}$ de la mezcla sensora con proporciones: $4.5 \%$ ionóforo, $37.9 \%$ DBBP, $0.8 \%$ NaTPB y $56.8 \%$ PVC y se deja polimerizar por evaporación a $40{ }^{\circ} \mathrm{C}$ por 24 horas. La membrana sensora se acondiciona en $\mathrm{Hg}$ (II) $0.5 \mathrm{~mol} \mathrm{~L}^{-1}$ por 5 días, después de la primera prueba el electrodo se coloca en solución pH 3.0 ajustada con $\mathrm{HCl} 0.1 \mathrm{~mol} \mathrm{~L}^{-1}$ e $\mathrm{NaOH} 0.1 \mathrm{~mol} \mathrm{~L}^{-1}$.

El análisis para mercurio(II) se realizó por cronopotenciometría a corriente nula en un potenciostato Epsilon CASi EW-8111. Se utilizó un sistema de dos electrodos, como electrodo de trabajo el aquí propuesto y $\mathrm{Ag} / \mathrm{AgCl}$ (Metrohm 6.0736.110) como referencia externa. Todas las pruebas se realizaron en una celda electroquímica convencional con $10 \mathrm{~mL}$ de solución $\mathrm{HCl} / \mathrm{NaOH}$ pH 3.0. En el esquema de la celda electroquímica (Figura 5.1) se puede observar que se elimina la solución interna de referencia y el electrodo de referencia interno. 


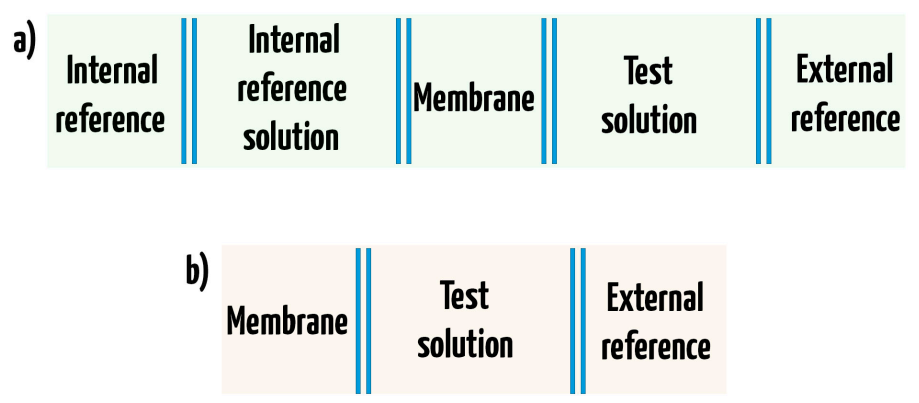

Figura 5.1. (a) Esquema de la celda electroquímica que utiliza el diseño de los ISE clásicos. (b) Esquema utilizando el diseño propuesto.

\subsection{RESULTADOS Y DISCUSIÓN}

\subsubsection{CALIBRACIÓN}

La selectividad está intrínsecamente relacionada con la naturaleza del elemento de reconocimiento, por esto el incesante empeño por diseñar más y mejores elementos receptores. Por otra parte, las demás características también dependen de la naturaleza del ionóforo, pero no en su totalidad. Un sensor químico costa de dos partes muy bien diferenciadas, un elemento de reconocimiento molecular o iónico, y un elemento instrumental denominado transductor. El transductor es el encargado de traducir la información generada en la reacción de reconocimiento en una señal útil y en último término del dominio eléctrico. De este elemento de transducción también dependen las características de un ISE. Por ello en este capítulo se emplean las características electroquímicas del grafito para demostrar su factible utilidad como transductor dentro del diseño de estos sensores. En la Figura 5.2 se muestra el esquema del diseño clásico de 
los ISE en contraste con el diseño aquí mostrado, se puede ver que se elimina la necesidad de una referencia interna y de una solución de referencia.

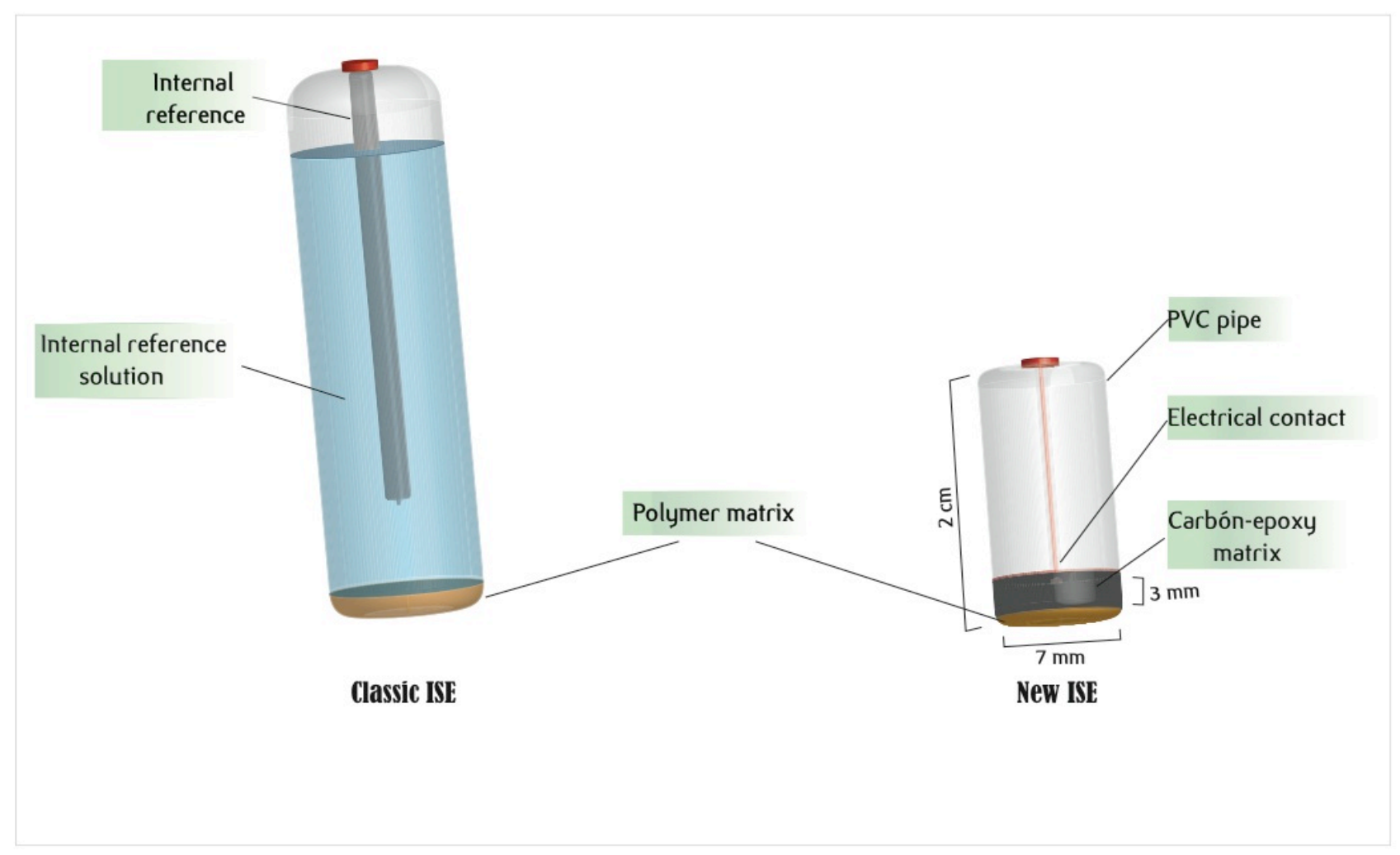

Figura 5.2. (a) Representación de un ESI convencional con electrodo de referencia interna y solución interna. (b) Diseño del ESI aquí propuesto.

Se realizaron curvas de calibración potenciométrica en un intervalo de concentraciones de $\mathrm{Hg}$ (II) $\left(1,0 \times 10^{-6}\right.$ a $\left.1,0 \times 10^{-1} \mathrm{~mol} \mathrm{~L}^{-1}\right)$ en solución de $\mathrm{HCl} / \mathrm{NaOH}$ pH 3.0. Se construyeron 2 lotes del mismo sensor para comprobar la reproducibilidad de los resultados. En la Tabla 5.1 se muestran los parámetros analíticos de seis electrodos que fueron probados. En la Figura 5.3 se 
muestra el promedio de las curvas de calibración $(n=6)$ con un coeficiente de variación menor al $1 \%$.

Tabla 5.1. Parámetros analíticos de las curvas de calibración realizados a los distintos lotes.

\begin{tabular}{ccccc}
\hline ISE & $\begin{array}{c}\text { Pendiente } \\
(\mathbf{m V})\end{array}$ & $\mathbf{L D D ~ x 1 0}^{-5}\left(\mathbf{m o l ~ L}^{-1}\right)$ & Intervalo lineal $\left(\mathbf{m o l ~ L}^{-1}\right)$ & $\mathbf{r}^{2}$ \\
\hline 1 & $29.4 \pm 0.2$ & $2.48 \pm 0.02$ & $2.9 \times 10^{-5}-1.0 \times 10^{-1}$ & 0.9994 \\
2 & $29.4 \pm 0.2$ & $2.40 \pm 0.02$ & $2.9 \times 10^{-5}-1.0 \times 10^{-1}$ & 0.9993 \\
3 & $29.4 \pm 0.3$ & $2.70 \pm 0.03$ & $2.9 \times 10^{-5}-1.0 \times 10^{-1}$ & 0.9991 \\
4 & $29.8 \pm 0.3$ & $2.59 \pm 0.03$ & $2.9 \times 10^{-5}-1.0 \times 10^{-1}$ & 0.9990 \\
5 & $29.6 \pm 0.3$ & $2.64 \pm 0.03$ & $2.9 \times 10^{-5}-1.0 \times 10^{-1}$ & 0.9987 \\
6 & $29.7 \pm 0.2$ & $2.75 \pm 0.02$ & $2.9 \times 10^{-5}-1.0 \times 10^{-1}$ & 0.9993 \\
\hline
\end{tabular}

Se observa que en las seis curvas de calibración realizadas para distintos electrodos construidos en diferentes tiempos los parámetros analíticos son muy comparables ya que su variación es despreciable, cada pendiente está dentro de la desviación estándar de las demás, el intervalo lineal resulta ser el mismo para todos los casos, estos resultados demuestran la reproducibilidad del método de construcción de los ESI. Se obtuvo una pendiente promedio de $(29.5 \pm 0.2) \mathrm{mV}$ década $^{-1}$ y un límite de detección promedio de $(2.6 \pm 0.1) \times 10^{-5} \mathrm{~mol} \mathrm{~L}^{-1}$, que es comparable con lo reportado por Gupta et. al. ${ }^{181}$, que reporta una pendiente de $(29.0 \pm 0.1) \mathrm{mV}$ década $^{-1}$ y un intervalo lineal de $3.1 \times 10^{-5}-1.0 \times 10^{-1} \mathrm{~mol} \mathrm{~L}^{-1}$. 


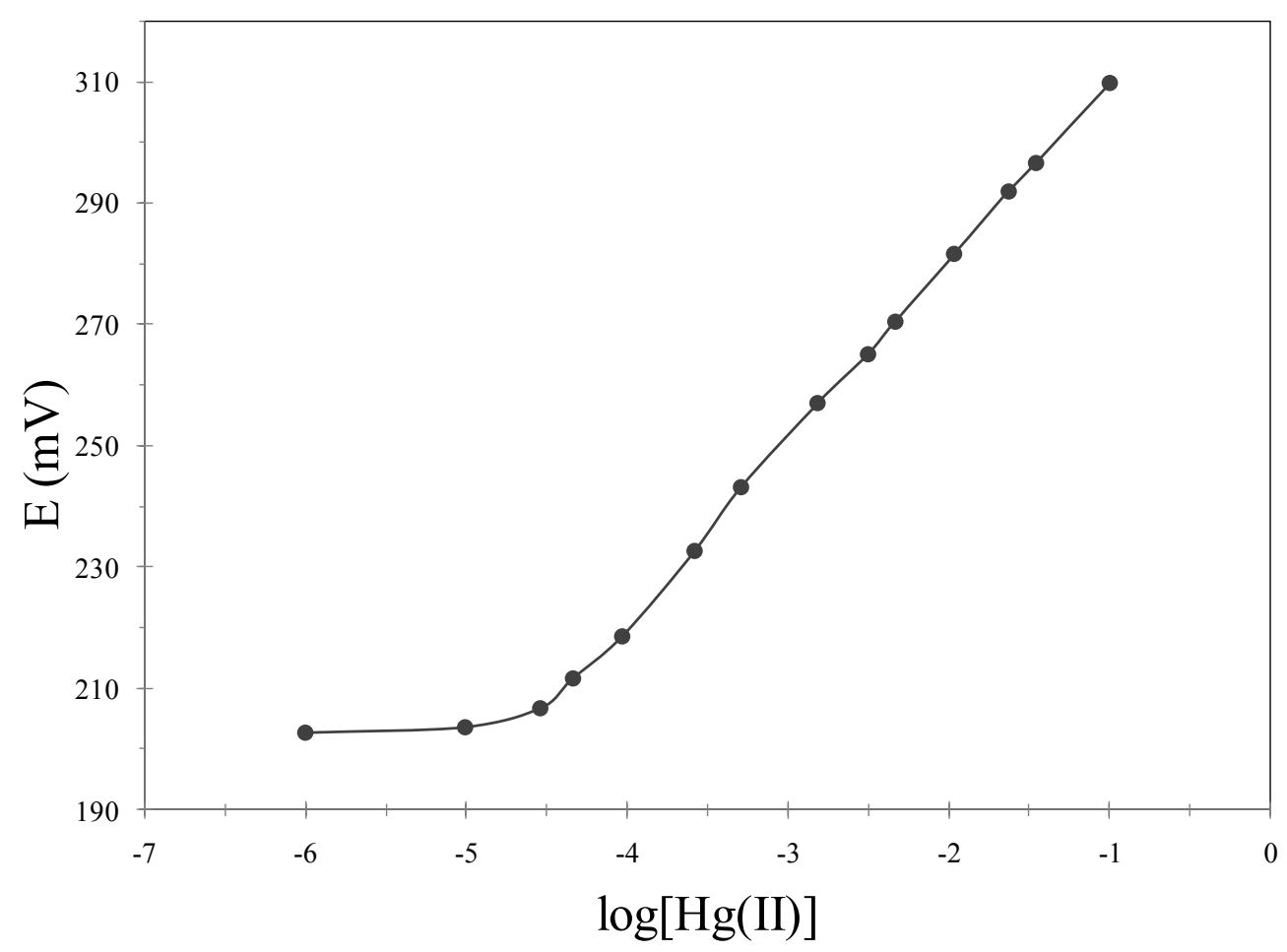

Figura 5.3. Promedio de las curvas de calibración potenciométricas $(n=6)$ para el ISE construido con la composición de la membrana reportada por Gupta et al y el nuevo diseño de contacto interno sólido propuesto aquí.

\subsubsection{TIEMPO DE RESPUESTA}

Como ya se dijo el tiempo de respuesta es un importante parámetro analítico para los ESI y en general para cualquier tipo de sensor químico independientemente de su diseño y naturaleza, ya que este parámetro está relacionado con la rapidez de la transducción de la señal generada en la reacción de reconocimiento, también claro relacionado con la rapidez de dicha reacción, y por lo tanto con la reversibilidad del proceso. Entonces aunque el tiempo de respuesta este relacionado 
con parámetros termodinámicos como la reversibilidad y la cinética del proceso de reconocimiento, también una buena transducción resulta imperativa. Por ello se toman las excelentes propiedades electroquímicas del grafito y se propone la matriz transductora carbónepoxy para un nuevo diseño de los ESI.

En la Figura 5.4 se muestra la variación del potencial de electrodo respecto al tiempo. Se realizaron adiciones de diferentes concentraciones de $\mathrm{Hg}(\mathrm{II})$ en solución de $\mathrm{HCl} / \mathrm{NaOH} \mathrm{pH} 3$, y se obtuvieron los potenciales en el tiempo. Se obtuvo un tiempo de respuesta de 5 segundos en contraste con los 15 segundos reportados por Gupta et al. Claramente la diferencia es significativa y no se puede atribuir a la naturaleza de la matriz polimérica ya que la composición es exactamente la misma, por lo tanto, esta diferencia puede ser atribuida a la eliminación del contacto líquido dado que un conductor iónico limita la rapidez en la transducción. Por otra parte en la matriz transductora, se aprovechan las ventajas electroquímicas del grafito respecto a conductores iónicos y metales nobles. 


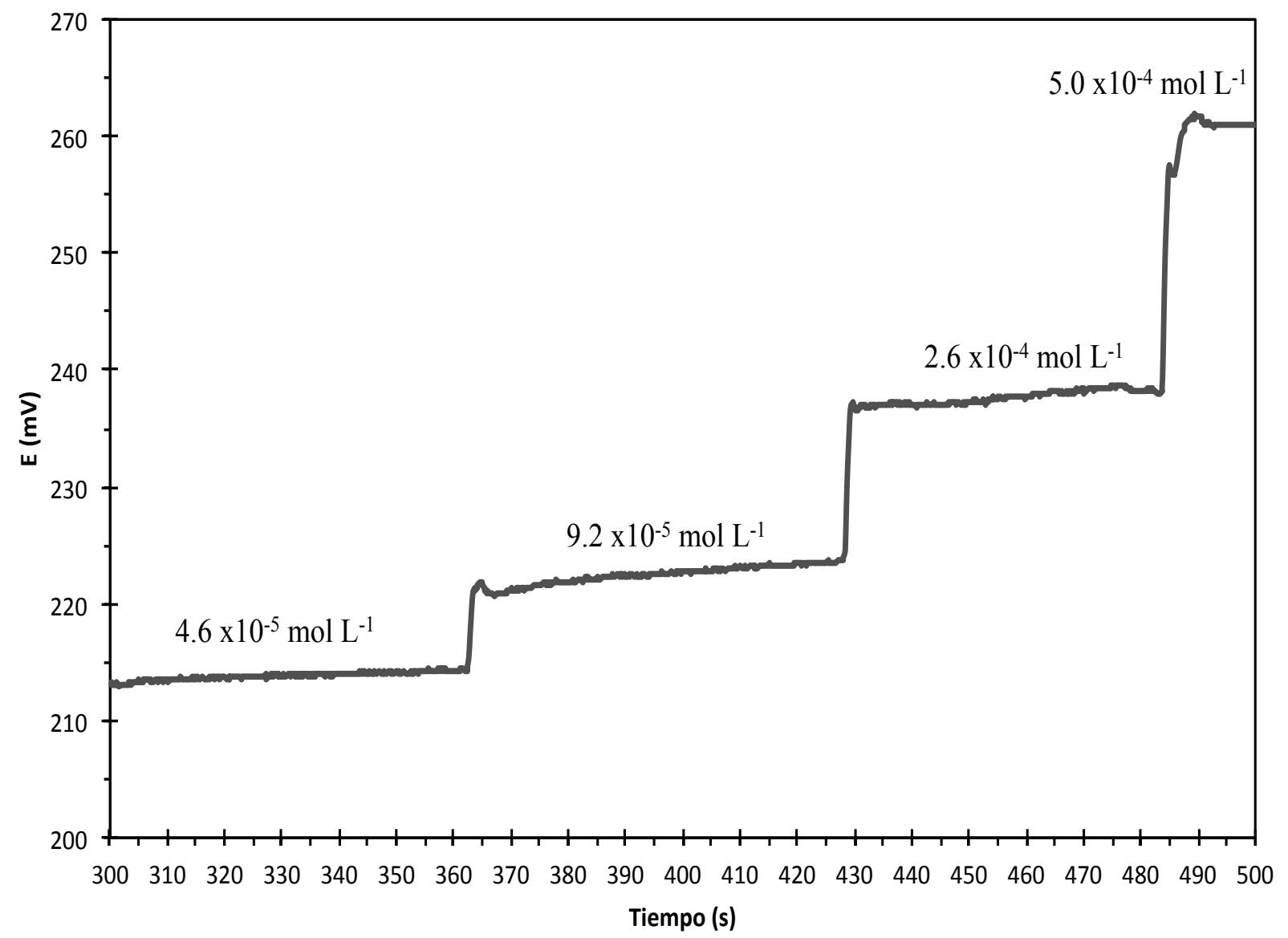

Figura 5.4. Variación del potencial de electrodo Vs tiempo.

\subsubsection{EFECTO DEL $\mathrm{pH}$}

La influencia del pH sobre el potencial del ISE construido se probó en solución de $\mathrm{Hg}\left(\mathrm{NO}_{3}\right)_{2}$ $1.0 \times 10^{-4} \mathrm{~mol} \mathrm{~L}^{-1}$ en un intervalo de $\mathrm{pH}$ de $0-7$ ajustado con solución de $\mathrm{HCl}$ y $\mathrm{NaOH} 0.1 \mathrm{~mol} \mathrm{~L}^{-1}$. Los resultados se muestran en la Figura 5.5, como puede observarse de $\mathrm{pH} 0$ a $\mathrm{pH} 2.5$ el potencial desciende hasta $219 \mathrm{mV}$ y así permanece hasta $\mathrm{pH} 4.7$ donde desciende nuevamente por la presencia de la especie insoluble $\mathrm{Hg}(\mathrm{OH})_{2}$ que coexiste en solución. Estos resultados 
concuerdan excelente con los reportados, lo que indica que el nuevo diseño del sensor funciona adecuadamente.

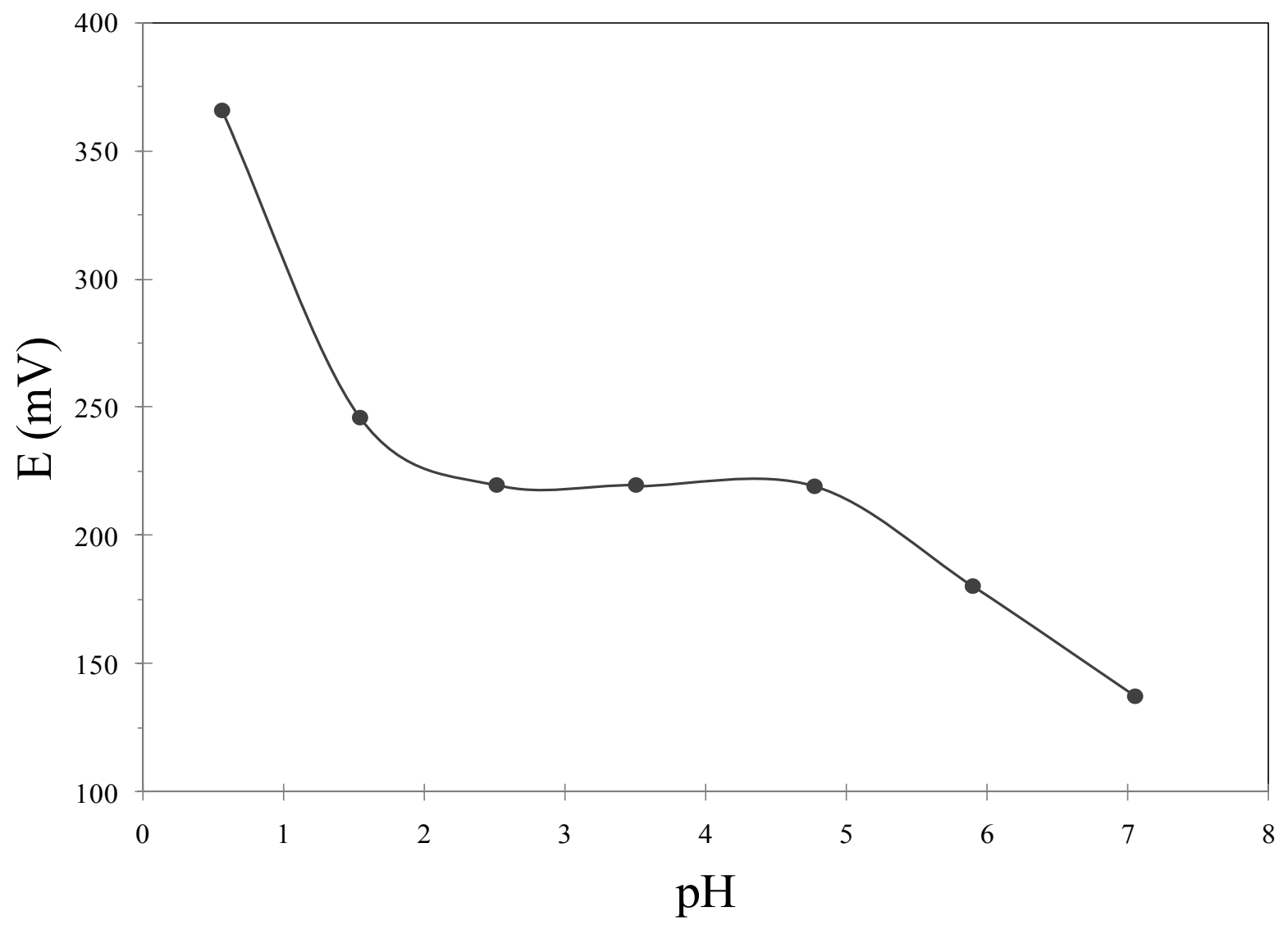

Figura 5.5. Influencia del pH sobre la respuesta del ISE.

\subsubsection{SELECTIVIDAD}

La selectividad es una de las propiedades más importante en las electrodos selectivos de iones, por esto se calcularon las constantes potenciométricas de selectividad utilizando el método de interferencia fija (FIM) el cual se basa en la ecuación de Nikolsky-Eisenman ${ }^{182,183,184 .}$ 


$$
E_{\mathrm{ISE}}=E^{0}+\frac{R T}{z_{i} F} \ln \left[a_{i}+\sum K_{i, j}^{P o t} a_{j}^{z_{i} / z_{j}}\right]
$$

donde ai , zi, aj y zj son las actividades y las cargas del ion principal e interferente respectivamente, los símbolos $\mathrm{R}, \mathrm{T}$ y $\mathrm{F}$ tienen el significado habitual. El término $\mathrm{E}^{\mathrm{o}}$ incorpora todas las contribuciones de potencial que son constantes y es distinto para cada ion estudiado y para cada tipo de electrodo. Utilizando el método FIM la solución del ion interferente se mantuvo constante en $1.0 \times 10^{-2} \mathrm{~mol} \mathrm{~L}^{-1}$ y la concentración del ion principal fue variada en solución pH 3 ajustado con $\mathrm{HCl} / \mathrm{NaOH} 0.1 \mathrm{~mol} \mathrm{~L}^{-1}$.

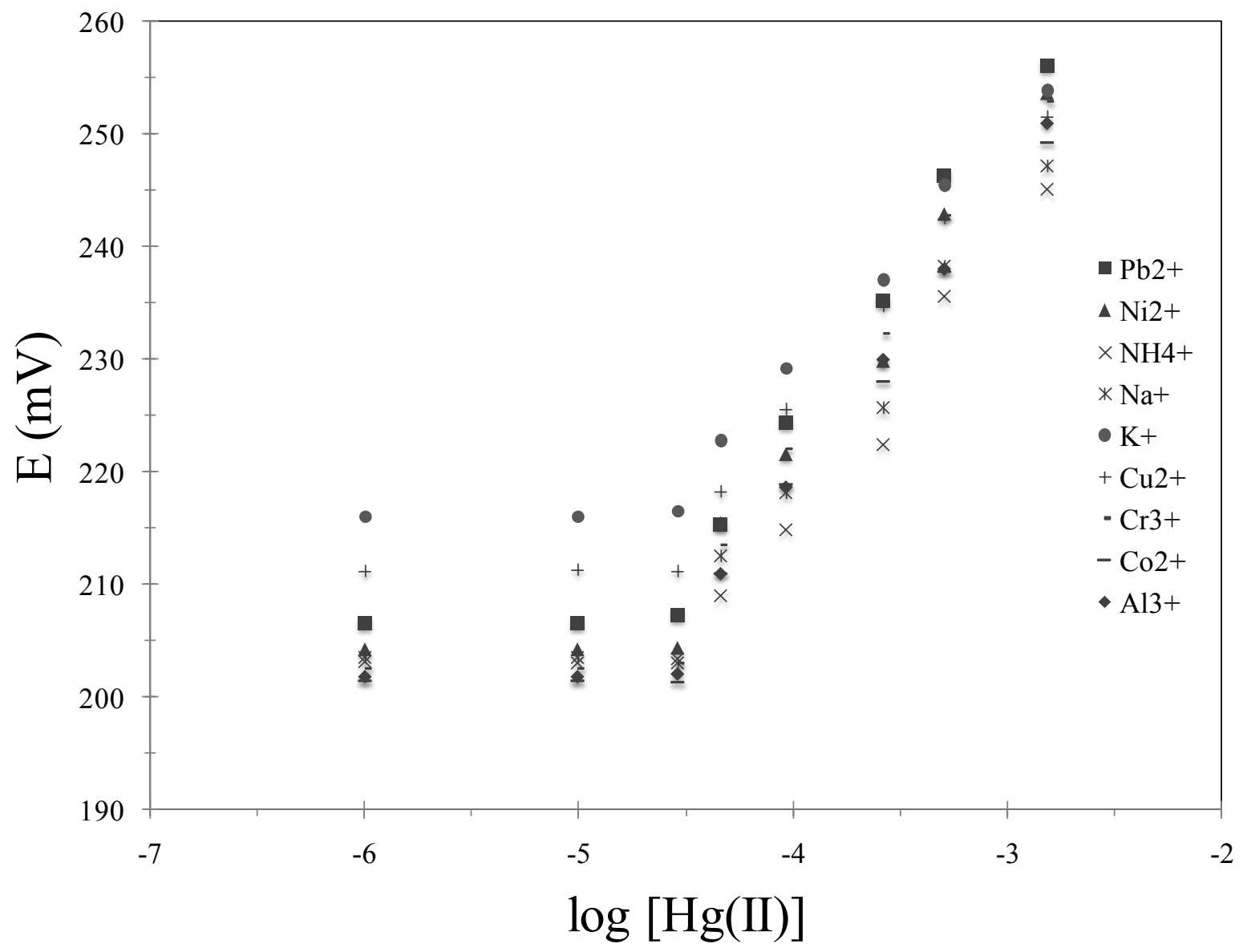

Figura 5.6. Respuesta potenciométrica del ISE a diferentes iones interferentes. 
En la Figura 5.6 se muestra la respuesta potenciométrica del sensor construido para diferentes iones interferentes como: $\mathrm{Pb}(\mathrm{II}), \mathrm{Ni}(\mathrm{II}), \mathrm{NH}_{4}^{+} \mathrm{Na}^{+}, \mathrm{K}^{+}, \mathrm{Cu}(\mathrm{II}), \mathrm{Cr}(\mathrm{III}), \mathrm{Co}(\mathrm{II})$ y $\mathrm{Al}(\mathrm{III})$. Como se observa las curvas siguieron la tendencia como para el ion principal $\mathrm{Hg}(\mathrm{II})$, en la Tabla 5.2 se muestran las constantes potenciométricas $K_{i, j}^{\text {Pot }}$ calculadas en contraste con las reportadas por Gupta et al.

Tabla 5.2. Constantes potenciométricas de selectividad calculadas con el método FIM.

\begin{tabular}{ccc}
\hline & $K_{\mathrm{Hg}(I I), j}^{P o t}$ & $\begin{array}{c}K_{\mathrm{Hg}(I I), j}^{\text {Pot }} \\
\text { reportadas por } \\
\text { Gupta et al. }\end{array}$ \\
\hline $\mathrm{Pb}^{2+}$ & $(3.1 \pm 0.2) \times 10^{-2}$ & $3.2 \times 10^{-2}$ \\
$\mathrm{Ni}^{2+}$ & $(2.9 \pm 0.1) \times 10^{-2}$ & $3.1 \times 10^{-2}$ \\
$\mathrm{Cu}^{2+}$ & $(2.5 \pm 0.1) \times 10^{-2}$ & $1.9 \times 10^{-2}$ \\
$\mathrm{Co}^{2+}$ & $(7.6 \pm 0.4) \times 10^{-2}$ & $7.9 \times 10^{-2}$ \\
$\mathrm{NH}_{4}^{+}$ & $(2.9 \pm 0.2) \times 10^{-1}$ & $5.0 \times 10^{-2}$ \\
$\mathrm{Na}^{+}$ & $(2.4 \pm 0.1) \times 10^{-1}$ & $1.7 \times 10^{-2}$ \\
$\mathrm{~K}^{+}$ & $(2.5 \pm 0.1) \times 10^{-1}$ & $7.0 \times 10^{-2}$ \\
$\mathrm{Cr}^{3+}$ & $(4.8 \pm 0.3) \times 10^{-4}$ & $1.4 \times 10^{-3}$ \\
$\mathrm{Al}^{3+}$ & $(5.3 \pm 0.3) \times 10^{-4}$ & $2.5 \times 10^{-3}$ \\
\hline
\end{tabular}


Haciendo una comparación de las constantes potenciométrica antes reportadas para la misma composición de la membrana sensora los valores difieren entre el diseño clásico y el aquí reportado y era de suponerse ya que mientras en el diseño clásico se tiene una solución de referencia de un lado de la membrana en el diseño aquí descrito no hay solución interna y tampoco electrodo de referencia, por lo que los potenciales medidos con un electrodo u otro no pueden ser iguales. La secuencia de selectividad utilizando el diseño aquí descrito es $\mathrm{Hg}(\mathrm{II})>\mathrm{NH}_{4}^{+}>\mathrm{K}^{+}>\mathrm{Na}^{+}>\mathrm{Co}(\mathrm{II})>\mathrm{Pb}(\mathrm{II})>\mathrm{Ni}(\mathrm{II})>\mathrm{Cu}(\mathrm{II})>\mathrm{Al}(\mathrm{III})>\mathrm{Cr}(\mathrm{III})$, mientras que la secuencia de selectividad con el diseño clásico es $\mathrm{Hg}(\mathrm{II})>\mathrm{Co}(\mathrm{II})>\mathrm{K}^{+}>\mathrm{NH}_{4}^{+}>\mathrm{Pb}(\mathrm{II})>\mathrm{Ni}(\mathrm{II})>\mathrm{Cu}(\mathrm{II})>\mathrm{Na}^{+}>$ $\mathrm{Al}(\mathrm{III})>\mathrm{Cr}(\mathrm{III})$. En ambas secuencias se nota que los iones divalentes tienen el mismo orden $[\mathrm{Co}(\mathrm{II})>\mathrm{Pb}(\mathrm{II})>\mathrm{Ni}(\mathrm{II})>\mathrm{Cu}(\mathrm{II})]$ y para cationes monovalentes y divalentes el orden de selectividad no concuerda, esto sucede por que el método FIM no estima correctamente las constantes de selectividad para iones con diferente carga que el ion principal ${ }^{185,186}$, ya que para el cálculo solo se toman en cuenta las actividades de los iones principal e interferente y sus cargas. Adicionalmente y para una mejor caracterización selectiva del sensor en la Tabla 5.3 se reportan las pendiente y límites de detección de las curvas de calibración para los iones $\mathrm{J}^{\mathrm{zj}}$ en presencia del ion $\mathrm{Hg}(\mathrm{II})$.

Tabla 5.3. Parámetros analíticos de las curvas de calibración para los iones interferentes en presencia de $\mathrm{Hg}(\mathrm{II})$.

\begin{tabular}{cccc}
\hline $\begin{array}{c}\text { ion interferente } \\
\mathrm{Jj}\end{array}$ & $\begin{array}{c}\text { Pendiente } \\
\left(\mathrm{mV} \text { década }^{-1}\right)\end{array}$ & $\begin{array}{c}\text { LDD } \\
\left(\mathrm{mol} \mathrm{L}^{-1}\right)\end{array}$ & $\mathrm{r}^{2}$ \\
\hline $\mathrm{Pb}^{2+}$ & $28.6 \pm 0.7$ & $(3.08 \pm 0.09) \times 10^{-6}$ & 0.9927 \\
$\mathrm{Ni}^{2+}$ & $28.3 \pm 0.9$ & $(2.92 \pm 0.09) \times 10^{-4}$ & 0.9814 \\
\hline
\end{tabular}




\begin{tabular}{cccc}
\hline $\mathrm{Cu}^{2+}$ & $23.0 \pm 0.8$ & $(2.59 \pm 0.08) \times 10^{-4}$ & 0.9944 \\
$\mathrm{Co}^{2+}$ & $29.2 \pm 1.0$ & $(3.12 \pm 0.08) \times 10^{-4}$ & 0.9904 \\
$\mathrm{NH}^{+}$ & $24.1 \pm 0.7$ & $(2.91 \pm 0.09) \times 10^{-5}$ & 0.9833 \\
$\mathrm{Na}^{+}$ & $24.7 \pm 1.0$ & $(2.44 \pm 0.01) \times 10^{-5}$ & 0.9804 \\
$\mathrm{~K}^{+}$ & $21.3 \pm 0.5$ & $(2.56 \pm 0.07) \times 10^{-5}$ & 0.9944 \\
$\mathrm{Cr}^{3+}$ & $28.1 \pm 1.6$ & $\left(2.25 \pm 0.09 \times 10^{-5}\right.$ & 0.9876 \\
$\mathrm{Al}^{3+}$ & $27.4 \pm 0.9$ & $(1.11 \pm 0.04) \times 10^{-4}$ & 0.9957 \\
\hline
\end{tabular}

Como se puede observar las pendientes de las curvas de calibración para los cationes divalentes está muy cerca de la Nernstiana al contrario de los iones monovalentes y trivalentes $\left(\right.$ excepto $\mathrm{Cr}^{3+}$ ) los cuales presentan una respuesta por de bajo de la esperada. Entonces tomando en cuenta que la pendiente de las curvas de calibración es el parámetro más importante en un ISE la secuencia de selectividad queda como: $\mathrm{Hg}(\mathrm{II})>\mathrm{Co}(\mathrm{II})>\mathrm{Pb}(\mathrm{II})>\mathrm{Ni}(\mathrm{II})>\mathrm{Cr}(\mathrm{III}))>\mathrm{Al}(\mathrm{III})>\mathrm{Na}^{+}>$ $\mathrm{NH}_{4}{ }^{+}>\mathrm{Cu}(\mathrm{II})>\mathrm{K}^{+}$, donde el orden del los cationes divalentes sigue el mismo orden que en el método FIM.

\subsection{CONCLUSIONES}

La incorporación de un contacto interno sólido trae importantes ventajas tanto en el diseño del sensor como en el manejo, ya que al ser eliminadas la referencia interna y la solución de referencia, la construcción y manipulación se hacen mas sencillas. También el modificar el diseño clásico permite miniaturizar los sensores favoreciendo su costo. La matriz carbón-epoxy resulta ser muy fácil de manejar y manipular, además de no ser costosa. Los resultados demostraron que la matriz carbón-epoxy puede sustituir eficientemente al diseño clásico en los 
ISE, ya que al funcionar como conductor electrónico la transducción de la señal es mas rápida comparada con un conductor iónico.

Los ISE expuestos a lo largo de esta tesis presentan mejores parámetros analíticos, ya que tienen mejores límites de detección y un más amplio intervalo de respuesta, también el intevalo de pH donde se pueden utilizar es más amplio, son más robustos ya que al construirlos se reducen considerablemente los costos de fabricación, además son mucho más fáciles de manipular, de construir y de almacenar. 


\section{CONCLUSIONES GENERALES}




\section{CONCLUSIONES GENERALES}

Los compuestos sintetizados se pudieron utilizar como ionóforos en la construcción de electrodos para la determinación selectiva de iones mercurio(II).

Se logró demostrar el buen funcionamiento del nuevo diseño de contacto sólido, ya que es muy comparable con un ESI clásico, con esta metodología de construcción <<que en principio es aplicable para un ESI selectivo a cualquier ion $>>$ se tienen numerosas ventajas, ya que al ser de contacto sólido se eliminan parámetros costosos y problemáticos, ya no es necesaria una referencia interna y támbien es eliminada la solución interna que tantos problemas acarrea, el diseño es fácil de construr, fácil de manejar y fácil de almacenar gracias a los materiales utilizados.

Se construyó un ESI para la determinación de iones $\mathrm{Hg}(\mathrm{II})$ utilizando el ionóforo PenDTF y el nuevo diseño de contacto sólido, este sensor presentó una respuesta Nernstiana y presentó ventajas en comparación con los ESI propuestos en la literatura para la determinación del mismo ion. Entre las ventajas se encuentra que no fue necesaria una membrana polimérica para la inmovilización de los ionóforos, en vez de esto se inmovilizó al ionóforo en la misma matriz de carbón-epoxy ahorrándonos la utilización de polímero, plastificante y otros aditivos, otra ventaja es que el ESI propuesto se puede regenerar con un simple tratamiento con papel abrasivo.

Los ionóforos restantes también fueron probados, pero esta vez en una membrana polimérica. Todos los compuestos presentaron respuesta lineal al incremento de iones $\mathrm{Hg}(\mathrm{II})$ en la celda, pero no todos presentaron una respuesta Nernstiana, por lo tanto, después de numerosos estudios se eligió al MetDTF como mejor ionóforo para la construcción de un nuevo ESI. El nuevo ESI presentó ventajas frente a otros ESI propuestos en la literatura ya que alcanzó límites de 
detección comparables con métodos instrumentales de analísis muy bien establecidos como lo es la electroforésis capilar o la espectrofotometría de absorción atómica. 


\section{PERSPECTIVAS}




\section{PERSPECTIVAS}

Este trabajo se pude tomar como base para el diseño de nuevos electrodos selectivos a iones $\mathrm{Hg}(\mathrm{II})$ ya que los ionóforos pueden usarse en la modificación de distintos electrodos, por ejemplo la matriz polimérica reportada en esta investigación podría ser usada para modificar electrodos impresos que utilizan un sistema de trasducción parecido al carbón-epoxy pero en pistas de grafito, también utilizan un solo electrodo de referencia. La modificación de electrodos impresos con la membrana polimérica daría como resultado ISE's más robustos, manejables, fáciles de construir y menos costosos. También podrían ser utilizados en FIA (flux inyection analysis) para la determinación continua de mercurio inorgánico en aguas reciduales por ejemplo. 


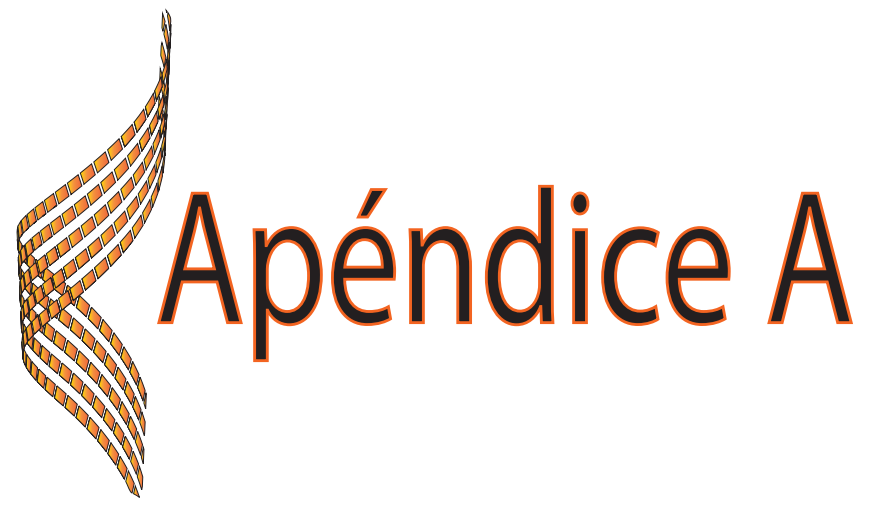




\section{Apéndice A}

\section{APÉNDICE A. Efecto de la fuerza iónica}

Los electrodos miden la actividad de los iones en solución, actividad que está relacionada con su concentración analítica, $\mathrm{C}_{\mathrm{i}}$, a través del coeficiente de actividad, mediante la ecuación:

$$
a_{i}=\gamma_{i} C_{i}
$$

Donde el coeficiente de actividad de electrolitos o iones, $\gamma_{i}$, es función de la fuerza iónica, I, que se define como:

$$
I=\frac{1}{2} \sum_{i=1}^{n} z_{i}^{2} C_{i}
$$

Donde $\mathrm{z}$ es la carga del ion considerado y $\mathrm{C}$ su concentración en mol L ${ }^{-1}$. Al llevar a cabo el trazado de las líneas de calibrado, deberá representarse la función: 


$$
E=E^{0}+\frac{R T}{z_{i} F} \ln a_{i}
$$

Donde $\mathrm{E}^{\mathrm{o}}$ es un término constante, que engloba diversos factores, como por ejemplo los potenciales de referencia, los potenciales de unión, etc. Al disponer sólo de concentraciones molares y representar la función como:

$$
E=E^{0}+\frac{R T}{z_{i} F} \ln C_{i}
$$

Se desprecia el término $\left(R T / z_{i} F\right) \ln \gamma_{i}$, ya que en realidad la ecuación correcta debería ser:

$$
E=E^{0}+\frac{R T}{z_{i} F} \ln \gamma_{i}+\frac{R T}{z_{i} F} \ln C_{i}
$$

Donde $\gamma_{i}$ varía con la fuerza iónica corrigiendo debidamente el valor de E, sobre todo a valores elevados de $\mathrm{C}_{\mathrm{i}}$. Para evitar este problema, lo que suele hacerse es mantener conastante la fuerza iónica. Como, por otra parte el potencial de la celda electroquímica es función de los potenciales de unión líquida además de los de membrana, es absolutamente necesario que se fije el valor de la fuerza iónica. 


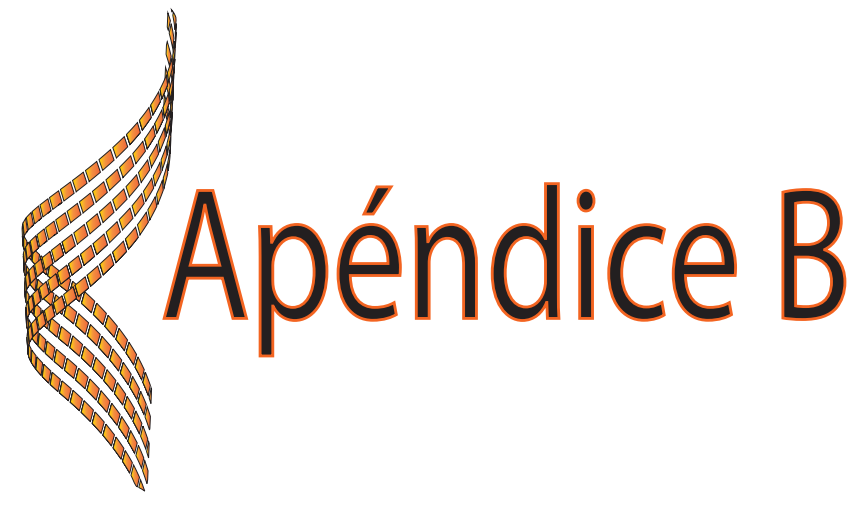




\section{Apéndice B}

\section{APÉNDICE B. Diseño factorial.}

Muchos químicos consideran las comparaciones estadísticas como métodos que solo se utilizan para evaluar los resultados de un experimento concluido. Aunque este es de hecho un área de aplicación esencial de la estadística, es también necesario ser consciente de la importancia de los conceptos estadísticos en la planificación y diseño de experimentos. El predecir errores sistemáticos y aleatorios, permite al analista hacer planes para después valorarlos. Es obvio que se espere identificar, antes de que empiece el experimento, en donde puede surgir el error dominante, y entonces intentar minimizarlo. Aunque los errores aleatorios nunca pueden eliminarse, en realidad pueden minimizarse prestando atención preferente a las técnicas experimentales.

Existen otros aspectos aún más sutiles del diseño experimental. En muchos análisis, se encuentra que una o más características deseables del método (por ejemplo sensibilidad, selectividad, etc.) dependerán de una serie de factores experimentales. Al diseñar el experimento, hay que pretender que la combinación óptima de estos factores se use de forma adecuada, y obtener la mejor sensibilidad, selectividad, etc. Aunque puedan estar implicados algunos experimentos preliminares o conocimientos previos, la optimización deberá realizarse (con el 
objeto de conservar recursos y tiempo) antes de que el método se utilice de forma rutinaria o se haga un uso generalizado del mismo.

El término diseño experimental se utiliza habitualmente para describir las etapas de: 1) Identificación de los factores que pueden influir en el resultado de un experimento, 2) Diseño del experimento de modo que se minimicen los efectos de los factores incontrolados, 3) Utilización del análisis estadístico para separar y evaluar los efectos de los diversos factores implicados.

Puesto que son muchos los factores que afectan a los resultados experimentales, pueden necesitarse diseños experimentales ciertamente complejos. La elección de los mejores niveles prácticos de estos factores, es decir, la optimización de las condiciones experimentales, requiere de un estudio detallado.

En muchas técnicas analíticas la respuesta del sistema de medida depende de una variedad de factores experimentales bajo el control del operador. Para una aplicación concreta es importante establecer los niveles de estos factores para asegurar que (por ejemplo) la sensibilidad sea tan alta como sea posible. El proceso de búsqueda de estos niveles óptimos del factor se conoce como optimización. No obstante, antes de comenzar con un proceso de optimización se determinan los factores y las interacciones que afectan de manera importante a la respuesta: es también valioso conocer qué factores tienen escaso o ningún efecto, de manera que no se desperdicien tiempo ni otros recursos en experimentos innecesarios.

Tales estudios utilizan un experimento factorial en el que cada factor tenga dos niveles, conocidos habitualmente por $<<$ alto $>>$ y $<<$ bajo $>>$. En el caso de una variable cuantitativa los términos $<<$ alto $>>$ y $<<$ bajo $>>$ tienen el significado usual. La elección exacta de los niveles se determina principalmente por la experiencia y el conocimiento del experimentador y las restricciones físicas del sistema, por ejemplo, en disolución acuosa sólo son practicables 
temperaturas en el intervalo $0-100^{\circ} \mathrm{C}$. Para una variable cualitativa, $<<$ alto $>>$ y $<<$ bajo $>>$ se refieren a condiciones diferentes. En esta Tesis se utiliza un diseño factorial de tres factores $\left(2^{3}\right)$ : A, B, C. Esto significa que hay $2 \times 2 \times 2=8$ combinaciones posibles de niveles de factores, como se muestra en la tabla E.1. Un signo más indica que el factor está en el nivel alto y un signo menos que está en el nivel bajo. La primera columna proporciona una notación utilizada a menudo para describir las combinaciones, donde la presencia de la correspondiente letra en minúscula indica que el factor está en el nivel alto y su ausencia que el factor está en el nivel bajo. El numero 1 se utiliza para indicar que todos los factores están en el nivel bajo.

Tabla B.1. Se muestran las posibles combinaciones de los factores para un diseño $2^{3}$.

\begin{tabular}{ccccc}
\hline Combinación & A & B & C & Respuesta \\
\hline $\mathbf{1}$ & - & - & - & $\mathbf{y}_{1}$ \\
a & + & - & - & $\mathbf{y}_{2}$ \\
b & - & + & - & $\mathbf{y}_{3}$ \\
c & - & - & + & $\mathbf{y}_{4}$ \\
bc & - & + & + & $\mathbf{y}_{5}$ \\
ac & + & - & + & $\mathbf{y}_{6}$ \\
ab & + & + & - & $\mathbf{y}_{7}$ \\
abc & + & + & + & $\mathbf{y}_{8}$ \\
\hline
\end{tabular}

Hay dos razones de peso para utilizar un diseño factorial en lugar de un clásico en experimentos que contrasten si la respuesta depende del nivel del factor:

1. El experimento factorial detecta y estima cualquier interacción que no puede hacer el experimento de un factor a la vez. 
2. Si los efectos de los factores son aditivos, entonces el diseño factorial necesita menos medidas que la aproximación clásica para proporcionar la misma precisión. En general, para $\mathrm{k}$ factores, una aproximación clásica supone $\mathrm{k}$ veces tantas medidas como en un diseño factorial con la misma precisión.

El análisis de varianza o ANOVA es una herramienta estadística que permite comparar simultáneamente varias medidas muestrales. Las medidas se comparan para establecer si son todas iguales (hipótesis nula), o si al menos una de ellas es distinta de las demás (hipótesis alternativa).

El nombre ANOVA hace alusión a que la comparación de dos medidas se realiza mediante el cálculo y la comparación de dos varianzas, pero se debe tener presente que el objetivo es comparar medidas no varianzas. La necesidad de aplicar un ANOVA es relativamente frecuente en el ámbito del laboratorio analítico.

Para la estimación de los efectos de cada factor y la interacción entre factores se utiliza un ANOVA y para que éste sea válido se deben satisfacer las siguientes condiciones:

1. El error aleatorio es el mismo para todas las combinaciones de los niveles de los factores.

2. Los errores aleatorios siguen distribuciones (aproximadamente) normales.

Cada una de las $\mathrm{N}$ medidas, $\mathrm{x}_{\mathrm{ij}}$, se clasifica bajo los términos niveles de los tratamientos y bloques. Utilizando los símbolos convencionales existen c niveles de tratamiento y $\mathrm{r}$ bloques, por tanto, $\mathrm{N}=$ cr. También aparecen los totales por fila $\left(\mathrm{T}_{1}, \mathrm{~T}_{2}\right.$, etc.) y por columna $\left(\mathrm{T}_{1}, \mathrm{~T}_{2}\right.$, etc.) y el total global, T, que se necesitan en los cálculos. 
Tabla B.2. Fórmulas ANOVA con interacción.

\begin{tabular}{ccc}
\hline $\begin{array}{c}\text { Fuente } \\
\text { de variación }\end{array}$ & Suma & Grados de libertad \\
\hline Entre filas & $\sum_{i} \frac{T_{i}^{2}}{n c}-C$ & $\mathrm{r}-1$ \\
Entre columnas & $\sum_{j} \frac{T_{j}^{2}}{n c}-C$ & $\mathrm{c}-1$ \\
Interacción & Por diferencia & Por diferencia \\
Residual & $\sum_{i j k} X_{i j k}^{2}-\sum_{i j} \frac{T_{i j}^{2}}{n}$ & $\mathrm{rc}$ (n-1) \\
Total & $\sum_{i j k} X_{i j k}^{2}-C$ & $\mathrm{rcn}-1$ \\
\hline
\end{tabular}

No obstante un problema que aparece en un experimento factorial completo es que el número de experimentos requerido crese rápidamente con el número de factores: para $\mathrm{k}$ factores en dos niveles con dos réplicas para cada combinación de valores, son necesarios $2^{\mathrm{k}+1}$ experimentos, por ejemplo, para cinco factores, 64 experimentos. Cuando hay más de tres factores es posible una simplificación, suponiendo que sean despreciables las interacciones de orden tres y superiores. Las sumas de cuadrados correspondientes a estas interacciones se pueden entonces combinar para proporcionar una estimación de la suma de los cuadrados residual, no siendo necesarias más réplicas. La lógica de esta aproximación es que los efectos de orden más grande son normalmente más pequeños que los efectos principales y que los efectos de interacción entre los dos factores. Si las interacciones de orden superior se pueden suponer insignificantes, una fracción adecuada de todas las posibles combinaciones de los niveles del factor es suficiente para 
proporcionar una estimación de los efectos principales y de interacción de dos factores. Dicho diseño experimental se llama diseño factorial fraccional o incompleto.

Otro problema al utilizar un diseño factorial para determinar qué factores tienen un efecto significativo sobre la respuesta es que, para factores que son variables continuas, el efecto depende de los niveles alto y bajo utilizados. Si estos niveles están ${ }^{187}$ excesivamente cerca una de otro, se puede obtener que el efecto del factor correspondiente no sea significativo a pesar del hecho de que sobre el intervalo posible completo de los niveles del factor, el efecto de este factor no sea despreciable. Por otra parte, si los niveles se alejan bastante pueden caer a ambos lados de un máximo, y aún así dar lugar a una diferencia en respuesta que no sea significativa. 


\section{REFERENCIAS}




\section{REFERENCIAS}

${ }^{1}$ S. Engst, S.M. Miller, Biochemistry 1999, 38, 3519-3529.

${ }^{2}$ H. Qian, L. Sahlman, P.O. Eriksson, C. Hambraeus, U. Edlund, I. Sethson, Biochemistry 1998, 37, 9316-9322.

${ }^{3}$ R.K. Mahajan, R. Kaur, V. Bhalla, M. Kumar, T. Hattori, S. Miyano, Sens. Actuators B 2008, 130, $290-294$.

${ }^{4}$ R.D. Maeco, G. Clarke, B. Pejctc, Electroanalysis 1987, 19, 1987-2001.

${ }^{5}$ X. Yu, Z. Zhou, Y. Wang, Y. Liu, Q. Xie, D. Xiao, Sens. Actuators B 2007, 123, 352-358.

${ }^{6}$ Z.L. Peng, F. Qu, Q. Song, J.M. Lin, Electrophoresis 2005, 26, 3333-3340.

${ }^{7}$ H. Hintelmann, R.D. Evans, J.V. Villeneuve, J. Anal. At. Spectrom. 1995, 10, 619-624.

${ }^{8}$ P. Buhlmann, E. Pretsch, E. Bakker, Chem. Rev. 1998, 98, 1593-1687.

${ }^{9}$ F. Bakhtiarzadeh, S.A. Ghani, J. Electroanal. Chem. 2008, 624, 139-143.

${ }^{10}$ Alan Ceresa, Eric Bakker, Bodo Hattendorf, Detlef Gunther, Erno Pretsch, Anal. Chem. 2001, 73, $343-351$.

${ }^{11}$ E. Bakker, P. Buhlmann, E. Pretsch, Chem. Rev. 1997, 97, 3083-3132.

${ }^{12}$ Moore, C., Pressmann, B. C., Biochem. Biophys. Res. Commun. 1964, 15, 562.

${ }^{13}$ Stefanac, Z., Simon, W., Chimia. 1966, 20, 436.

${ }^{14}$ Pedersen, C. J., J. Am. Chem. Soc. 1967, 89, 2495-2496

${ }^{15}$ Dietrich, B., Lehn, J., M. Sauvage, J. P., Tetrahedron Lett. 1969, 34, 2885.

${ }^{16}$ Shatkay, A., J. Phys. Chem. 1967, 71, 3858.

${ }^{17}$ Shatkay, A. Anal. Chem. 1967, 39, 1056.

${ }^{18}$ Ross, J. W. Science 1967, 156, 1378.

${ }^{19}$ E. Bakker, P. Buhlmann, E. Pretsch, Chem. Rev. 1997, 97, 3083-3132

${ }^{20}$ P. Buhlmann, E. Pretsch, E. Bakker, Chem. Rev. 1998, 98, 1593-1687.

${ }^{21}$ E. Bakker, P. Buhlmann, E. Pretsch, Chem. Rev. 1997, 97, 3083-3132.

${ }^{22}$ Ciani, S. M.; Eisenman, G.; Szabo, G. J. Membrane Biol. 1969, 1, 1.

${ }^{23}$ Pungor, E. Pure Appl. Chem. 1992, 64, 503.

${ }^{24}$ Umezawa, K.; Lin, X. M.; Nishizawa, S.; Sugawara, M.; Umezawa, Y. Anal. Chim. Acta 1993, $282,247$.

${ }^{25}$ Bakker, E.; Nagele, M.; Schaller, U.; Pretsch, E. Electroanalysis 1995, 7, 817.

${ }^{26}$ Horvai, G.; Graf, E.; To'th, K.; Pungor, E.; Buck, R. P. Anal. Chem. 1986, 58, 2735.

${ }^{27}$ van den Berg, A.; van der Wal, P. D.; Skowronska-Ptasinska, M.; Sudholter, E. J. R.; Reinhoudt, D. N.; P., B. Anal. Chem. 1987, 59, 2827.

${ }^{28}$ Buhlmann, P.; Yajima, S.; Tohda, K.; Umezawa, K.; Nishizawa, S.; Umezawa, Y. Electroanalysis 1995, 7, 
811.

${ }^{29}$ Buhlmann, P.; Yajima, S.; Tohda, K.; Umezawa, Y. Electrochim. Acta 1995, 40, 3021.

${ }^{30}$ Henderson, L. J. J. Biol. Chem. 1921, 46, 411.

${ }^{31}$ E. Bakker, P. Buhlmann, E. Pretsch, Chem. Rev. 1997, 97, 3083-3132.

${ }^{32}$ Pungor, E. Pure Appl. Chem. 1992, 64, 503.

${ }^{33}$ Bakker, E.; Nagele, M.; Schaller, U.; Pretsch, E. Electroanalysis 1995, 7, 817.

${ }^{34}$ Buhlmann, P.; Yajima, S.; Tohda, K.; Umezawa, K.; Nishizawa, S.; Umezawa, Y. Electroanalysis 1995, 7 , 811.

${ }^{35}$ Buhlmann, P.; Yajima, S.; Tohda, K.; Umezawa, Y. Electrochim. Acta 1995, 40, 3021.

${ }^{36}$ Buhlmann, P.; Yajima, S.; Tohda, K.; Umezawa, K.; Nishizawa, S.; Umezawa, Y. Electroanalysis 1995, 7 , 811.

${ }^{37}$ E. Bakker, P. Buhlmann, E. Pretsch, Chem. Rev. 1997, 97, 3083-3132.

${ }^{38}$ E. Bakker, E. Pretsch. Anal. Chem. 2002, 74, 420A.

${ }^{39}$ R. P. Buck, E. Lindner. Pure Appl. Chem. 1994, 66, 2527.

${ }^{40}$ R. P. Buck, E. Lindner. Pure Appl. Chem. 1994, 66, 2527.

${ }^{41}$ A. Ceresa, T. Sokalski, E. Pretsch. J. Electroanal. Chem. 2001, 501, 70.

${ }^{42}$ T. Zwickl, T. Sokalski, E. Pretsch. Electroanalysis 1999, 11, 673.

${ }^{43}$ E. Lindner, R. E. Gyurcsanyi, R. P. Buck. Electroanalysis 1999, 11, 695.

${ }^{44}$ E. Pergel, R. E. Gyurcsányi, K. Tóth, E. Lindner. Anal. Chem. 2001, 73, 4249.

${ }^{45}$ K. Toth, K. Stulik, W. Kutner, Z. Feher, E. Lindner. Pure Appl. Chem. 2004, 76, 1119.

${ }^{46}$ A. Cadogan, Z. Q. Gao, A. Lewenstam, A. Ivaska, D. Diamond. Anal. Chem. 1992, 64, 2496.

${ }^{47}$ K. Y. Chumbimuni-Torres, N. Rubinova, A. Radu, L. T. Kubota, E. Bakker. Anal. Chem. 2006, 78, 1318.

${ }^{48}$ R. E. Gyurcsanyi, A. S. Nyback, K. Toth, G. Nagy, A. Ivaska. Analyst 1998, 123, 1339.

${ }^{49}$ R. E. Gyurcsanyi, N. Rangisetty, S. Clifton, B. D. Pendley, E. Lindner. Talanta 2004, 63, 89.

${ }^{50}$ A. Michalska, A. Hulanicki, A. Lewenstam. Microchem. J. 1997, 57, 59.

${ }^{51}$ J. Sutter, E. Lindner, R. Gyurcsanyi, E. Pretsch. Anal. Bioanal. Chem. 2004, 380, 7.

${ }^{52}$ R. Zielinska, E. Mulik, A. Michalska, S. Achmatowick, M. Maj-Zurawska. Anal. Chim. Acta 2002, 451, 243.

${ }^{53}$ E. Bakker, P. Buhlmann, E. Pretsch. Electroanalysis 1999, 11, 915. 
${ }^{54}$ R. Zielinska, E. Mulik, A. Michalska, S. Achmatowick, M. Maj-Zurawska. Anal. Chim. Acta 2002, $451,243$.

55 A. C. Ion, E. Bakker, E. Pretsch. Anal. Chim. Acta 2001, 440, 71.

${ }^{56}$ Ruzicka, J.; Tjell, J. C. Anal. Chim. Acta 1970, 51, 1.

${ }^{57}$ Baiulescu, G. E.; Cosofret, V. V. Talanta 1976, 23, 677.

${ }^{58}$ Szczepaniak, W.; Olesky, J. Anal. Chim. Acta 1986, 189, 237.

${ }^{59}$ Lai, M.-T.; Shih, J.-S. Analyst 1986, 111, 891.

${ }^{60}$ Masuda, Y.; Sekido, E. Bunseki Kagaku 1990, 39, 683.

${ }^{61}$ Pietraszkiewicz, M.; Gasiorowski, R.; Brzózka, Z. J. Inclusion Phenom. Mol. Recognit. Chem. 1990, 9, 259.

${ }^{62}$ Brzozka, Z.; Petraszkiewicz, M. Electroanalysis 1991, 3, 855.

${ }^{63}$ R. K. Mahajan, R. Kaur, I. Kaur, V. Sharma, M. Kumar, Analytical Sciences 2004, 20, 811-814.

${ }^{64}$ B. Patel, A. Kumar, S. K. Menon, J. Incl. Phenom. Macrocycl. Chem. 2009, 64, 101-108.

${ }^{65}$ V. K. Gupta, B. Sethi, E. A. Aharma, S. Agarwal, A. Bharti, J. Molecular Liquids 2013, 177, 114-118.

${ }^{66}$ A. K. S. Gupta, R. C. Srivastava, S. S. Parma, Can. J. Chem. 1967, 45, 2293.

${ }^{67}$ S. E. Livingstone, A. E. Mihkelson, Inorg. Chem. 1970, 9, 2545.

${ }^{68}$ J. A., S. Gill, R. S. J. Kowalski, N. A. Bailey, H. Adams, K. W. Lumbard, M.A. Murphy, J. Chem. Soc., Dalton Trans. 1982, 493-504.

${ }^{69}$ Y. R. Li, G. Pereira, M. Kasrai, P. R. Norton, Tribol Lett. 2008, 29, 201-211.

${ }^{70}$ A. Morina, A. Neville, J Phys D: Appl. Phys. 2007, 40, 5476-5487.

${ }^{71}$ H. F. Maltez, D. L. G. Borges, E. Carasek, B. Welz, A. J. Curtius Talanta 2008, 74, 800-805.

${ }^{72}$ I. M. Dittert, T. A. Maranhão, D.L G. Borges, M. A. Vieira, B. Welz, A. J. Curtius Talanta 2007, 72, 17861790.

${ }^{73}$ M. Oleinikova, C. González, M. Valiente, M. Muñoz Polyhedron 1999, 18, 3353- 3359.

${ }^{74}$ S. Wingefors, Acta Chem. Scand. 1980, A34, 327.

75 M. E. Páez-Hernández, K. Aguilar-Arteaga, M. Valiente, M. T. Ramírez Silva, M Romero Romo, M. Pardavé, Anal. Bioanal. Chem. 2004, 380(4), 690-697.

${ }^{76}$ M. W. Matlock, B. S. Howerton, M. A. Van Aelstyn, F. L. Nordstrom and D. A. Atwood, Environ. Sci. Technol. 2002, 36, 1636-1639.

${ }^{77}$ R. Singh, S. N. Tandon, Talanta 1997, 44, 843-848.

${ }^{78}$ C. Fontas, M. Hidalgo, V. Salvado, E. Antico, Anal. Chim. Acta 2005, 547, 255- 261. 
79 P.G. Francisco, A.R. José Guadalupe, G.V. Carlos Andrés, P.H. Maria Elena, A.L. Noemí, Struct. Chem. 2010, 21, 191-196.

${ }^{80}$ S. Engst, S.M. Miller, Biochemistry 1999, 38, 3519-3529.

${ }^{81}$ H. Qian, L. Sahlman, P.O. Eriksson, C. Hambraeus, U. Edlund, I. Sethson, Biochemistry 1998, 37, 93169322.

${ }^{82}$ R.K. Mahajan, R. Kaur, V. Bhalla, M. Kumar, T. Hattori, S. Miyano, Sens. Actuators B 2008, 130, $290-294$.

${ }^{83}$ I. Bontidean, A. Mortari, S. Leth, N.L. Brown, U. Karlson, M.M. Larsen, J. Vangronsveld, P. Corbisier, E. Csoregi, Environ. Pollut. 2004, 131, 255-262.

${ }^{84}$ J.L. Hobman, N.L. Brown, Metal Ions in Biological Systems, Marcel Dekker, New York, 1997.

85 J.L. Hobman, J.R. Wilson, N.L. Brown, D.R. Lovley, Environmental Microbe Metal Interactions, ASM Press, Herndon, 2000.

${ }^{86}$ R.D. Maeco, G. Clarke, B. Pejctc, Electroanalysis 1987, 19, 1987-2001.

${ }^{87}$ X. Yu, Z. Zhou, Y. Wang, Y. Liu, Q. Xie, D. Xiao, Sens. Actuators B 2007, 123, 352-358.

${ }^{88}$ Z.L. Peng, F. Qu, Q. Song, J.M. Lin, Electrophoresis 2005, 26, 3333-3340.

${ }^{89}$ H. Hintelmann, R.D. Evans, J.V. Villeneuve, J. Anal. At. Spectrom. 1995, 10, 619-624.

${ }^{90}$ I. Palchetti, S. Majid, A. Kicela, G. Marrazza, M. Mascini, Int. J. Environ. Anal. Chem. 2003, 83, 701-711.

${ }^{91}$ P. Buhlmann, E. Pretsch, E. Bakker, Chem. Rev. 1998, 98, 1593-1687.

${ }^{92}$ F. Bakhtiarzadeh, S.A. Ghani, J. Electroanal. Chem. 2008, 624, 139-143.

${ }^{93}$ V.K. Gupta, S. Chandra, H. Lang, Talanta 2005, 66, 575-580.

${ }^{94}$ A.K. Singh, R.P. Singh, S. Mehtab, J. Inclusion Phenom. Macrocyclic Chem. 2008, 60, 9-15.

${ }^{95}$ A.A. Khan, Inamuddin, T. Akhtar, Anal. Sci. 2008, 24, 881-887.

${ }^{96}$ N. Fiol, F. de la Torre, P. Demeyere, A. Florido, I. Villaescusa, Sens. Actuators B 2007, 122, $187-194$.

${ }^{97}$ V.K. Gupta, A.K. Singh, A. Al Khayat, B. Gupta, Anal. Chim. Acta 2007, 590, 81-90.

${ }^{98}$ R.K. Mahajan, I. Kaur, T.S. Lobana, Talanta 2003, 59, 101-105

${ }^{99}$ L. Perez-Marin, E. Otazo-Sanchez, G. Macedo-Miranda, P. Avila-Perez, J.A. Chamaro, H.L Lopez-Valdivia, Analyst 2000, 125, 1787-1790.

${ }^{100}$ R.K. Mahajan, R. Kaur, I. Kaur, V. Sharma, M. Kumar, Anal. Sci. 2004, 20, 811-814.

${ }^{101}$ M. Castro, J. Cruz, E. Otazo, L.P. Marın, J. Phys. Chem. 2003, A 107, 9000-9007.

${ }^{102}$ L.P. Marın, M. Castro, E. Otazo, G.A. Cisneros, Int. J. Quantum Chem. 2000, 80, 609-622.

103 P.G. Francisco, G.V. Carlos Andrés, A.R. José Guadalupe, P.H. María Elena, A.L. Noemi, R.S. María Teresa, Sep. Sci. Technol. Accepted. 
104 M.H. Mashhadizadeh, M. Talakesh, M. Peste, A. Momeni, H. Hamidian, M. Mazlumd, Electroanalysis 2006, 18, 2174-2179.

${ }^{105}$ R.K. Mahajan, R.K. Puri, A. Marwaha, I. Kaur, M.P. Mahajan, J. Hazard. Mater. 2009, 167, $237-243$.

106 P.G. Francisco, G.V. Carlos Andrés, A.R. José Guadalupe, P.H. María Elena, A.L. Noemi, R.S. María Teresa, Sep. Sci. Technol. Accepted.

107 IUPAC, Analytical chemistry division, Commission on Analytical Nomenclature, Recommendations for nomenclature of ion-selective electrodes, Pure Appl. Chem. 1976, 48, 127-142.

${ }^{108}$ Y. Umezawa, K. Umezawa, H. Sato, Pure Appl. Chem. 1995, 67, 507-518.

${ }^{109}$ A. Rojas-Hernández, M.T. Ramírez-Silva, J.G. Ibañez, I. González, J. Electrochem. Soc. 1991, 138 (2), $365-$ 371.

${ }^{110}$ A. Rojas-Hernández, M.T. Ramírez-Silva, I. González, J.G. Ibañez. Anal. Chim. Acta 1991, $246,435-441$.

${ }^{111}$ B. Patel, A. Kumar, S.K. Menon, J. Inclusion Phenom. Macrocyclic Chem. 2009, 64, 101-108.

${ }^{112}$ Y. Yang, X. Cao, M. Surowiec, R.A. Bartsch, Tetrahedron 2010, 66, 447-454.

${ }^{113}$ B. Palkiran, A.D. Ozel, S. Palayan, E. Canel, U. Ocak, E. Kilic, Monatsh. Chem. 2010, 141, 829-839.

${ }^{114}$ H. Bingol, E. Kocabas, E. Zor, A. Coskum, Talanta 2010, 82, 1538-1542.

${ }^{115}$ M. Hosseine, M. Rahimi, H.B. Sodeghi, Int. J. Environ. Anal. Chem. 2009, 89, 407-422.

${ }^{116}$ Y. Kim, J. Kim, K.N. Kim, S. Chang, T.D. Chung, Anal. Sci. 2009, 25, 567-570.

${ }^{117}$ H.M. Abu-Shawish, J. Hazard. Mater. 2009, 167, 602-608.

${ }^{118}$ R.K. Mahan, R.K. Puri, A. Marwaha, I. Kaur, M.P. Mahajan, J. Hazard. Mater. 2009,167, $237-243$.

${ }^{119}$ M.K. Rofouei, M. Mohammadi, M.B. Gholivand, Mater. Sci. Eng. 2009, A 29, 2154-2159.

${ }^{120}$ R.K. Puri, V. Kumar, M.P. Mahajan, R.K. Mahajan, J. Inclusion Phenom. Macrocyclic Chem. 2011, 69, 263273.

${ }^{121}$ Global Mercury Hotspots - New evidence reveals mercury contamination regularly exceeds health advisory levels in humans and fish worldwide, A Publication By The Biodiversity Research Institute and IPEN, January 9, 2013.

${ }^{122}$ Bontidean, I.; Mortari, A.; Leth, S.; Brown, N.L.; Karlson, U.; Larsen, M.M.; Vangronsveld, J.; Corbisier, P.; Csoregi E. Environ. Pollut. 2004, 131, 255-262.

${ }^{123}$ Hobman, J.L.; Brown N.L. Metal Ions in Biological Systems, Marcel Dekker: New York, 1997.

${ }^{124}$ Hobman, J.L.; Wilson, J.R.; Brown, N.L.; Lovley, D.R. Environmental Microbe Metal Interactions, ASM Press: Herndon, 2000.

${ }^{125}$ Castro, M.; Cruz, J.; Otazo, E.; Marın, L.P. J. Phys. Chem. A, 2003, 107, 9000-9007.

${ }^{126}$ Marın, L.P.; Castro, M.; Otazo, E.; Cisneros, G.A. Int. J. Quantum Chem. 2000, 80, 609-622.

${ }^{127}$ Mahajan, R.K.; Kaur, R.; Bhalla, V.; Kumar, M.; Hattori, T.; Miyano, S. Sens. Actuators, B 2008, 130, $290-$ 294. 
${ }^{128}$ Escudero, L.B.; Olsima, R.A.; Wuilloud, R.G. Talanta 2013, 116, 133-140.

${ }^{129}$ Hintelmann, H.; Evans, R.D.; Villeneuve, J.V. J. Anal. At. Spectrom. 1995, 10, 619-624.

${ }^{130}$ Peng Z.L.; Qu, F.; Song, Q.; Lin, J.M. Electrophoresis 2005, 26, 3333-3340.

${ }^{131}$ Martín-Yerga, D.; González-García, M.B.; Costa-García, A. Talanta 2013, 116, 1091-1104.

${ }^{132}$ Buhlmann, P.; Pretsch, E.; Bakker, E. Chem. Rev. 1998, 98, 1593-1687.

${ }^{133}$ Gupta, V.K.; Singh, A.K.; Al Khayat, A.; Gupta, B. Anal. Chim. Acta 2007, 590, 81-90.

${ }^{134}$ Mahajan, R.K; Kaur, I.; Lobana, T.S. Talanta 2003, 59, 101-105.

${ }^{135}$ Abu-Shawish, H.M. J. Hazard. Mater. 2009, 167, 602-608.

${ }^{136}$ Jumal, J.; Yamin, B.M.; Ahmad, M.; Heng, L.Y. APCBEE Procedia 2012, 3, 116-123.

${ }^{137}$ Perez-Marin, L.; Otazo-Sanchez, E.; Macedo-Miranda, G.; Avila-Perez, P.; Chamaro, J.A.; Lopez-Valdivia, H.L. Analyst 2000, 125, 1787-1790.

${ }^{138}$ Gupta, V.K.; Sethi, B.; A. Sharma, R.; Agarwal, S.; Bharti, A. J. Mol. Liq. 3013, 177, 114-118.

${ }^{139}$ Mahajan, R.K.; Kaur, R.; Kaur, I.; Sharma, V.; Kumar, M. Anal. Sci. 2004, 20, 811-814.

${ }^{140}$ Kim, Y.; Kim, J.; Kim, K.N.; Chang, S-K.; Chung, T.D. Anal. Sci. 2009, 25, 567-570.

${ }^{141}$ Yang, Y.; Cao, X.; Surowiec, M.; Bartsch, R.A. Tetrahedron 2010, 66, 447-454.

142 Puri, R.K.; Kumar, V.; Mahajan, M.P.; Mahajan, R.K. J. Inclusion Phenom. Macrocyclic Chem. 2011, 69, 263-273.

${ }^{143}$ Dalkiran, B.; Ozel, A.D.; Parlayan, S.; Canel, E.; Ocak, U.; Kilic, E. Monatsh. Chem. 2010, 141, 829-839.

${ }^{144}$ Yu, X.; Zhou, Z.; Wang, Y.; Liu, Y.; Xie, Q.; Xiao, D. Sens. Actuators, B 2007, 123, 352-358.

145 Tyagi, S.; Agarwal, H.; Ikram, S. Water Sci. Technol. 2010, 613, 693-704.

${ }^{146}$ Li, Y.; Wei, F.; Lu, Y.; He, S.; Zhao, L.; Zeng, X. Dyes Pigm. 2013, 96, 424-429.

${ }^{147}$ Chu, K-H.; Zhou, Y.; Fang, Y.; Wang, L-H.; Li, Y-J.; Yao, C. Dyes Pigm. 2013, 98, 339-346.

${ }^{148}$ Bingol, H.; Kocabas, E.; Zor, E.; Coskun, A. Talanta 2010, 82, 1538-1542.

${ }^{149}$ Yang, Y.; Cao, X.; Surowiec, M.; Bartsch, R.A. Tetrahedron 2010, 66, 447-454.

${ }^{150}$ Isaad, J.; Achari, A.E. Tetrahedron 2013, 69, 4866-4872.

${ }^{151}$ Li, Y.; Huang, H.; Li, Y.; Su, X. Sens. Actuators, B 2013, 188, 772-777.

${ }^{152}$ Li, F.; Wang, J.; Lai, Y.; Wu, C.; Sun. S.; He, Y.; Ma, H. Biosens. Bioelectron. 2013, 39, 82-87.

153 Juarez-Gomez, J.; Perez-Garcia, F.; Ramirez-Silva, M.T.; Rojas-Hernandez, A.; Galan-Vidal, C.A.; PaezHernandez, M.E. Talanta 2013, 114, 235-242.

154 IUPAC, Analytical chemistry division, Commission on Analytical Nomenclature,Recommendations for nomenclature of ion-selective electrodes, Pure Appl. Chem. 1976, 48, 127-142. 
${ }^{155}$ Umezawa, Y.; Umezawa, K.; Sato, H. Pure Appl. Chem. 1995, 67, 507-518.

${ }^{156}$ Bakker, E.; Pretsch, E.; Buhlmann, P. Anal. Chem. 2000, 72, 1127-1133.

${ }^{157}$ Bakker, E.; Buhlmann, P.; Pretsch, E. Chem. Rev. 1997, 97, 3083-3132.

158 Juarez-Gomez, J.; Perez-Garcia, F.; Ramirez-Silva, M.T.; Rojas-Hernandez, A.; Galan-Vidal, C.A.; PaezHernandez, M.E. Talanta 2013, 114, 235-242.

${ }^{159}$ Escudero, L.B.; Olsima, R.A.; Wuilloud, R.G. Talanta 2013, 116, 133-140.

${ }^{160}$ Gupta, V.K.; Sethi, B.; A. Sharma, R.; Agarwal, S.; Bharti, A. J. Mol. Liq. 3013, 177, 114-118.

${ }^{161}$ Patel, B.; Kumar, A.; Menon, S.K. J. Incl. PhenomMacrocycl. Chem. 2009, 64, 101-108.

${ }^{162}$ Isaad, J.; Achari, A.E. Tetrahedron 2013, 69, 4866-4872.

${ }^{163}$ Li, Y.; Huang, H.; Li, Y.; Su, X. Sens. Actuators, B 2013, 188, 772-777.

${ }^{164}$ Rajabi, H.R.; Roushani, M.; Shamsipur, M. J. Electroanal. Chem. 2013, 693, 16-22.

${ }^{165}$ Zhang, Y.; Zhao, H.; Wu, Z.; Wue, Y.; Zhang, X.; He, Y.; Li, X.; Yuan, Z. Biosens. Bioelectron. 2013, 48, 180-187.

${ }^{166}$ I. Svancara, K. Vytras, J. Barek, J. Zima, Anal. Chem. 2001, 31, 311-345.

${ }^{167}$ K. Kalcher, J. M. Kauffmann, J. Wang, I. Svancara, K. Vytras, C. Neuhold, Z. Yang, Electroanalysis 1995, 7, 5-22.

${ }^{168}$ P. Buhlmann, E. Pretsch, E. Bakker, Chem. Rev. 1998, 98, 1593-1687.

${ }^{169}$ M. Cano, B. Palenzuela, R. Rodrígues-Amaro, Portugaliae Electrochimica Acta 2007, 25, 67-78.

${ }^{170}$ H. M. Abu-Shawish, J. Hazard. Mater. 2009, 167, 602-608.

${ }^{171}$ Y. M. Issa, H. Ibrahim, O. R. Shehab, J. Electroanal. Chem. 2012, 666, 11-18.

${ }^{172}$ S. Shahrokhian, M. K. Amini, R. Kia, S. Tangestaninejad, Anal. Chem. 2000, 72, 956-962.

${ }^{173}$ M. Fouskaki, N. A. Chaniotakis, Anal. Chem. 2005, 77, 1780-1784.

${ }^{174}$ S. Yu, F. Li, T. Yin, Y. Liu, D. Pan, W. Qin, Anal. Chim. Acta 2011, 702, 195-198.

${ }^{175}$ G. Lefevre, J. Bessiere, A. Walcarius, Sens. Actuators B 1999, 59, 113-117.

${ }^{176}$ J. S. Yeom, M. S. Won, Y. B. Shim, J. Electroanal. Chem. 1999, 463, 16.

${ }^{177}$ K. Gupta, S. Chandra, S. Agarwal, Indian J. Chem. 2003, 24a, 813-818.

${ }^{178}$ M. F. Mousavi, N. Alizadeh, M. Shamsipur, N. Zohari, Sens. Actuators B 2000, 66, 98-100.

${ }^{179}$ M. F. Mousavi, N. Alizadeh, M. Shamsipur, N. Zohari, Anal. Chim. Acta 2000, 414, 189-194.

${ }^{180}$ C. Hamamci, H. Hosgoren, S. Erdogan, Talanta 1998, 47, 229-236.

${ }^{181}$ V. K. Gupta, S. Chandra, S. Agarwal, Indian J. Chem. 2003, 24a, 813-818. 
${ }^{182}$ IUPAC, Pure Appl. Chem. 1976, 48, 127-142.

${ }^{183}$ Y. Umezawa, K. Umezawa, H. Sato, Pure Appl. Chem. 1995, 67, 507-518.

${ }^{184}$ E. Lindner, Y. Umezawa, Pure Appl. Chem. 2008, 80, 85-104.

${ }^{185}$ E. Bakker, E. Pretsch, P. Buhlmann, Anal. Chem. 2000, 72, 1127-1133.

${ }^{186}$ E. Bakker, P. Buhlmann, E. Pretsch, Chem. Rev. 1997, 97, 3083-3132. 


\section{ARTÍCULO PUBLICADO}




\section{Solid-contact $\mathrm{Hg}(\mathrm{II})$-selective electrode based on a carbon-epoxy composite containing a new dithiophosphate-based ionophore}

\section{J. Juarez-Gomez a , F. Perez-Garcia ${ }^{\text {a }}$, M.T. Ramirez-Silva ${ }^{\text {a,* }}$, A. Rojas-Hernandez ${ }^{\text {a }}$, C.A. Galan-Vidal ${ }^{\text {b }}$, M.E. Paez-Hernandez ${ }^{\text {b }}$}

a Universidad Autónoma Metropolitana-Iztapalapa, Departamento de Química, Área de Química Analítica, San Rafael Atlixco 186, Col. Vicentina, Del. Iztapalapa. C.P. 09340 México DF, México

${ }^{\mathrm{b}}$ Universidad Autónoma del Estado de Hidalgo, Centro de Investigaciones Químicas, Km. 4.5, Carretera Pachuca-Tulancingo, Col. Carboneras, Mineral de la Reforma, Hidalgo C.P. 42184, México

\section{A R T I C L E I N F O}

\section{Article history:}

Received 26 March 2013

Received in revised form

2 May 2013

Accepted 9 May 2013

Available online 16 May 2013

Keywords:

ISE

Ionophore

Mercury (II)

Selectivity

Potentiometric titration
A B S T R A C T

The experimental results herein concern a novel ion-selective electrode manufactured with an inner solid contact based on a new ligand $0, \mathrm{O}^{\prime}$-(2,2'-biphenylene)dithiophosphate pentyl (PenDTF). This electrode displays high selectivity toward the $\mathrm{Hg}(\mathrm{II})$ ion even in the presence of different divalent ions. The electrode also exhibited a good Nernstian response to $\mathrm{Hg}(\mathrm{II})\left(33.7 \pm 1.0 \mathrm{mV}\right.$ decade $\left.^{-1}\right)$ over an ample concentration range $\left(5.3 \times 10^{-7}-1.0 \times 10^{-2} \mathrm{~mol} \mathrm{~L}^{-1}\right)$, with a detection limit of $(6.1 \pm 1.7) \times 10^{-7} \mathrm{~mol} \mathrm{~L}^{-1}$. As a result of design, the electrode can be used for many experiments, simply renewing its contact surface with emery paper, without giving rise to a significant response deviation, exhibiting a variation coefficient of $3 \%$, capable of being tested within the $0-5 \mathrm{pH}$ interval. The electrode was used satisfactorily as indicating electrode during the potentiometric titration of $\mathrm{Hg}(\mathrm{II})$ ions with EDTA.

(c) 2013 Elsevier B.V. All rights reserved.

\section{Introduction}

Mercury is generally found at very low concentrations in the environment and can be easily absorbed by the human being and other living organisms $[1,2]$, where its bioaccumulation is highly toxic for the kidneys, causing also neurological damages, paralysis and even problems during pregnancy. Further, it can be found in diverse chemical forms, although the elemental and the inorganic forms can be transformed through biological systems in the organic form in short-chained alkyl compounds, which are well known to be responsible of damages to the central nervous system [3]. From the middle ages, mercury salts have been widely used for various human activities. For instance in medicine, they have been used as ointments for skin and eyes. More recently, mercury compounds are used in diuretics, dental amalgams, antiseptics etc. [4-6].

Due to the considerable impact that the ion has on the environment and on human health, there exists an imperative necessity to develop new chemical methods for accurate determination $[7,8]$ and effective removal $[9,10]$ of small mercury concentrations.

\footnotetext{
Corresponding author. Tel.: +52555804 46 70; fax: +525558044666.

E-mail addresses: mtrs218@xanum.uam.mx mtrs218@hotmail.com (M.T. Ramirez-Silva).

0039-9140/\$ - see front matter ๔ 2013 Elsevier B.V. All rights reserved. http://dx.doi.org/10.1016/j.talanta.2013.05.013
}

There have been classical spectroscopy methods used for the determination of mercury, characterized for having a low detection limit, like atomic absorption spectroscopy (AAS), inductively coupled plasma mass spectrometry (ICP-MS) and capillary electrophoresis, among others, although they are costly techniques that cannot be readily used in field analysis $[11,12]$.

Among the different techniques within the analytical field for metal ions, electroanalysis is considered one of the most powerful ones; however, the use of conventional electrochemical cells and several voluminous electrodes, poses a problem for recording measurements in part due to a fairly large electrolyte solution volume required [13].

The potentiometric sensors offer several advantages such as: low cost, ease of fabrication, low detection limits, wide response range, reasonable selectivity, among others $[14,15]$. Diverse reports can be found in the literature concerning ion-selective electrodes (ISE) that used neutral ligands for $\mathrm{Hg}$ (II) detection, from which it becomes obvious that the contact surface configuration varies [1619], as in some cases the ligand is encapsulated within a polymer membrane, to decrease its loss in the electrode. However, it has been found also that the surface undergoes irreversible damages when $\mathrm{Hg}(\mathrm{II})$ is added to the solution, which induces the wrong responses after each experiment [19].

From the first $\mathrm{Hg}$ (II) ion-selective electrode that was based on 1,4-dithio-12-crown-4 reported by Lai and Shih [20], other 
different ionophores have been synthesized and used for $\mathrm{Hg}$ (II) selective detection, ligands such as thiols [21], Schiff's bases [22], thioureas [23], and calixarene's derivatives [24], among others have been used under conditions where the nitrate ion $\left(\mathrm{NO}_{3}{ }^{-}\right)$was the predominant anionic species, due to the low solubility of the mercury species with counter ions different to nitrate.

It is known that $\mathrm{Hg}$ (II) shows significant affinity toward ligands containing sulfur atoms, which explains their successful use as ionophores for this metal; such affinity has been corroborated using DFT (Density Functional Theory) calculations $[25,26]$.

This research work presents the potentiometric studies for $\mathrm{Hg}$ (II) detection with a new ion-selective electrode with an inner solid contact; the composite for its construction was a 1:1 epoxy resin/graphite mix, into which the ionophore $0,0^{\prime}-\left(2,2^{\prime}\right.$-biphenylene)dithiophosphate pentyl (PenDTF) was mixed in. The electrode can be put to use immediately after every experiment during a given sequence, simply renewing its contact surface through controlled-grit size abrasive traditional treatment.

The chemical compound used as ionophore (PenDTF, see Fig. 1) was dithiophosphate-type, which has displayed, during extraction and transport studies, high selectivity to $\mathrm{Hg}$ (II) before different metal ions [27]. Chemically, the PenDTF ligand is a neutra compound bearing donating atoms like sulfur, with free electron pairs that can be coordinated to the metal ions; also this is hydrophobic ligand, which favors the potentiometric studies in aqueous media. In view of the aforementioned, in this work a novel ISE electrode for the selective and quantitative determination of $\mathrm{Hg}(\mathrm{II})$ is presented.

\section{Experimental}

\subsection{Reagents and solutions}

All substances used were analytical grade reagents. The PenDTF compound was synthesized following the method reported in [28]. 2,2'-biphenol, toluene, 2-propanol anhydrous, phosphorus pentasulfide and 1-bromopentane were all from Sigma-Aldrich. Deionized water with $18 \mathrm{M} \Omega$ resistivity from a Milli-Q Millipore equipment was used to prepare all solutions. The support electrolyte solution was $1.0 \mathrm{~mol} \mathrm{~L}^{-1} \mathrm{HClO}_{4}$ (J.T. Baker) along with $\mathrm{Hg}$ $\left(\mathrm{NO}_{3}\right)_{2} \cdot \mathrm{H}_{2} \mathrm{O}$ (Merck) to prepare the corresponding ions solutions.

\subsection{Electrode design and its potential measurements}

Araldite M resin and hardener HY 5162 (Ciba-Geigy) were mixed in a small glass container, along with graphite and PenDTF ligand as indicated in Table 1; after mixing thoroughly all constituents until a homogeneous paste was achieved, a small portion

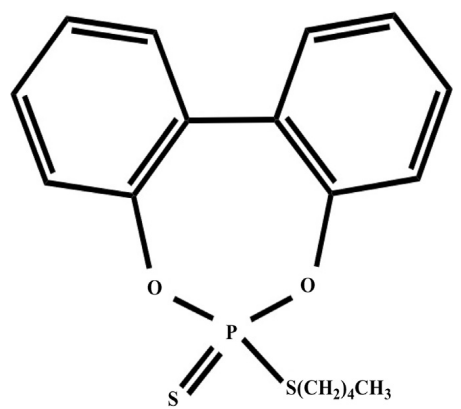

Fig. 1. Ligand structure $O, O^{\prime}-\left(2,2^{\prime}\right.$-bifenilen)dithiophosphate pentyl (PenDTF) was inserted in a PVC tube with an approximate $7 \mathrm{~mm}$ ID. A copper contact bar was placed centered in the paste to provide electrical connection, subsequently the device, $\mathrm{Hg}$ (II)-ISE-PenDTF, was left to rest for $24 \mathrm{~h}$ at $40{ }^{\circ} \mathrm{C}$. Thereafter, the resulting electrode was immerse-conditioned in $1.0 \times 10^{-3} \mathrm{~mol} \mathrm{~L}^{-1} \mathrm{Hg}\left(\mathrm{NO}_{3}\right)_{2}$ for $5 \mathrm{~min}$ prior running each test.

$\mathrm{The} \mathrm{Hg}(\mathrm{II})$ analysis was undertaken by means of chronopotentiometry at the null current potential using an Epsilon BASi EW8111 potentiostat. A two-electrode set-up was used where the working electrode was that obtained mixing in the ligand PenDTF while a saturated $\mathrm{Ag} / \mathrm{AgCl}$ (Metrohm 6.0736.110) worked as reference. All experiments were carried out with the aid of a conventional glass electrochemical cell holding $10 \mathrm{~mL}$ of $1.0 \mathrm{~mol} \mathrm{~L}^{-1} \mathrm{HClO}_{4}$ (J.T. Baker)

\subsection{Potentiometric titration}

Titrations of $\mathrm{Hg}$ (II) with ethylenediaminetetraacetic acid, EDTA (Sigma), were carried out using as working electrode a mercury cup electrode (M221Hg-9, Radiometer analytical) or the $\mathrm{Hg}(\mathrm{II})$ ISE-PenDTF in acetate buffer prepared with acetic acid and sodium acetate (Baker Analyzed), along a reference electrode of $\mathrm{Ag} / \mathrm{AgCl}$ (Metrohm 6.0736.110).

\section{Results and discussion}

\subsection{Synthesis}

PenDTF compound was synthesized following the method reported in [28], Fig. 2 shows the nuclear magnetic resonance spectrum (NMR) of ${ }^{1} \mathrm{H}$ in $\mathrm{CDCl}_{3}$ at $25{ }^{\circ} \mathrm{C}$ : a NMR spectrometer Bruker DMX500 of $500 \mathrm{MHz}$ was used to perform the characterization. The chemical shifts for the aliphatic part below; $\delta(\mathrm{ppm}): 3.10$ (dt, $2 \mathrm{H}^{3} \mathrm{~J}_{\mathrm{H}-\mathrm{P}}=$ $19.25 \mathrm{~Hz}$ ), 1.78 (quintet, $\left.2 \mathrm{H}, \mathrm{CH}_{2}{ }^{\mathrm{a}}\right), 1.38\left(\mathrm{~m}, 2 \mathrm{H}, \mathrm{CH}_{2}{ }^{\mathrm{b}}\right), 1.38(\mathrm{~m}, 2 \mathrm{H}$ $\left.\mathrm{CH}_{2}{ }^{\mathrm{C}}\right)$, and $0.89\left(\mathrm{t}, 3 \mathrm{H}, \mathrm{CH}_{3}\right)$

\subsection{Optimization of the electrode's composition}

It is paramount for ion selective electrodes, ISE, that the recognition element is included in their composition, as well as to control precisely the additives used to build them because most of the relevant analytic parameters, like sensitivity and selectivity, depend largely on their nature. Therefore, different aspects of their composition were optimized, like the influence of the composite composition over the potentiometric response of the electrode, which was analyzed by means of factorial design type $2^{3}$ (two levels, three factors): the three factors analyzed were the resin, the hardener amount and the ionophore's concentration; the high level for each factor was 250,100 and $20 \mathrm{mg}$ whereas the low level per factor was 200,50 and $10 \mathrm{mg}$, respectively, thus keeping constant the graphite amount in the composite. Table 1 shows the composition of the different electrodes built along with their analytic parameters. The potentiometric response of the sensors was tested within a wide $\mathrm{Hg}$ (II) concentrations range from $1.0 \times 10^{-9}$ to $1.0 \times 10^{-2} \mathrm{~mol} \mathrm{~L}^{-1}$ in $1.0 \mathrm{~mol} \mathrm{~L}^{-1} \mathrm{HClO}_{4}$. Each test was performed quintuplicate to ensure reproducible results; a variation $(n=5)$.

From the data presented in Table 1 concerning the differen electrodes for the $\mathrm{Hg}$ (II)-ISE-PenDTF, it becomes clear that electrode 1 is the best sensor since it exhibits the best analytic features, like the best Nernstian behavior, the best sensitivity and an ample linearity range (see Fig. 3), which constitute the main reasons for choosing it as the working electrode for subsequent experiments.

A comparison of the influence of individual factors on the response levels of the sensors, through the proposed factorial 
Table 1

Factorial design applied to the electrode's composition.

\begin{tabular}{|c|c|c|c|c|c|c|c|}
\hline \multirow[t]{2}{*}{ Hg(II)-ISE-PenDTF ${ }^{a}$} & \multicolumn{4}{|c|}{ Composition of the electrode (mg) } & \multirow[t]{2}{*}{ Slope (mV decade $\left.{ }^{-1}\right)$} & \multirow[t]{2}{*}{ LOD $\left(\mathrm{mol} \mathrm{L}^{-1}\right)$} & \multirow[t]{2}{*}{ Linear interval $\left(\mathrm{mol} \mathrm{L}^{-1}\right.$} \\
\hline & Graphite & Resin & Hardener & Ionophore & & & \\
\hline 1 & 350 & 250 & 100 & 20 & $33.7 \pm 1.0$ & $6.1 \times 10^{-7} \pm 1.7 \times 10^{-7}$ & $5.3 \times 10^{-7}-1.0 \times 10^{-2}$ \\
\hline 2 & 350 & 250 & 50 & 20 & $146 \pm 27$ & $2.4 \times 10^{-5} \pm 5.5 \times 10^{-6}$ & $5.0 \times 10^{-5}-1.0 \times 10^{-2}$ \\
\hline 3 & 350 & 200 & 50 & 20 & $100 \pm 11$ & $6.7 \times 10^{-5} \pm 1.1 \times 10^{-5}$ & $9.2 \times 10^{-5}-1.0 \times 10^{-2}$ \\
\hline 4 & 350 & 200 & 50 & 10 & $71.0 \pm 8.0$ & $2.3 \times 10^{-5} \pm 3.5 \times 10^{-6}$ & $5.0 \times 10^{-5}-1.0 \times 10^{-2}$ \\
\hline 5 & 350 & 200 & 100 & 10 & $32.0 \pm 0.8$ & $1.9 \times 10^{-6} \pm 8.5 \times 10^{-7}$ & $3.6 \times 10^{-6}-1.0 \times 10^{-2}$ \\
\hline 6 & 350 & 250 & 100 & 10 & $38.0 \pm 2.5$ & $1.6 \times 10^{-4} \pm 4.1 \times 10^{-5}$ & $1.8 \times 10^{-4}-1.0 \times 10^{-2}$ \\
\hline 7 & 350 & 200 & 100 & 20 & $21.4 \pm 1.6$ & $1.0 \times 10^{-5} \pm 8.2 \times 10^{-7}$ & $1.6 \times 10^{-5}-1.0 \times 10^{-2}$ \\
\hline 8 & 350 & 250 & 50 & 10 & $94.5 \pm 7.1$ & $2.0 \times 10^{-5} \pm 6.5 \times 10^{-6}$ & $5.0 \times 10^{-5}-1.0 \times 10^{-2}$ \\
\hline
\end{tabular}

${ }^{\text {a }} \mathrm{Hg}$ (II)-ISE-PenDTF (ion- selective electrode Hg(II) based on PenDTF).

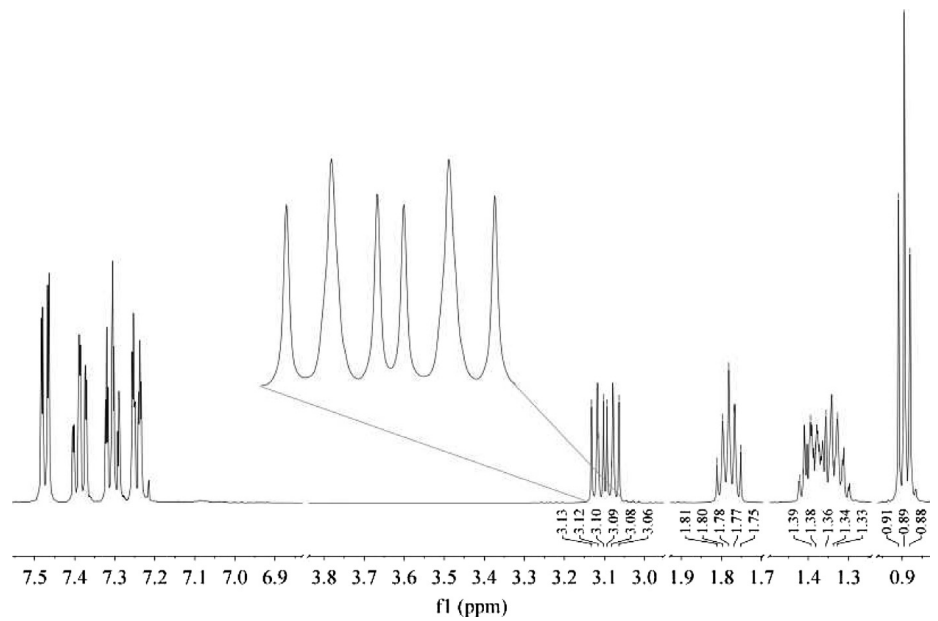

Fig. 2. ${ }^{1} \mathrm{H}$ NMR spectrum in $\mathrm{CDCl}_{3}$ for PenDTF.

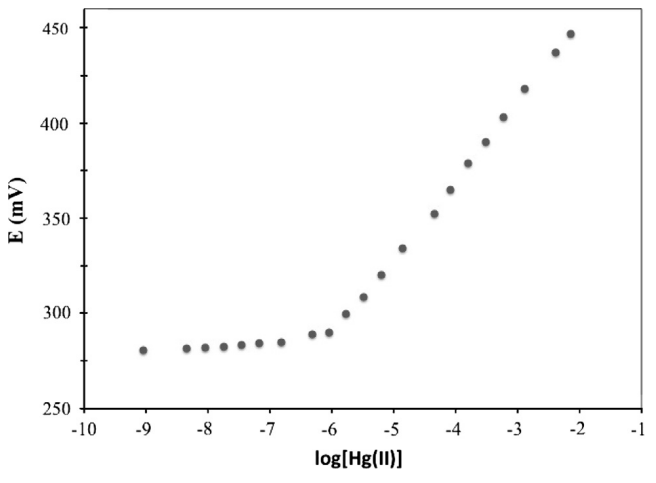

Fig. 3. Calibration curve of electrode Hg(II)-ISE-PenDTF 1.

design shows that for compositions 6 and 7, the ionophore is an important factor because the low level yields a concentration limit of detection (LOD, [29]) a decade lower with respect to the higher level. When comparing compositions 2, 3 and 8 a steep climb can be seen above those expected from the Nernst equation, that coincides with the low hardener level, focusing on the ionophore; meanwhile, the design shows that the lost slope level obtained is smaller than for the case of composition 8. Now, when comparing the sensors 1 and 5 that is where one gets the best analytical parameters; namely, that both ionophore levels have similar slopes, but with a decade fold the LOD concentration at the lowest level.

\subsection{Effect of $\mathrm{pH}$ on potential response of electrode}

The $\mathrm{pH}$ influence on the $\mathrm{Hg}$ (II)-ISE-PenDTF was determined using a $1.0 \times 10^{-3} \mathrm{~mol} \mathrm{~L}^{-1} \mathrm{Hg}\left(\mathrm{NO}_{3}\right)_{2}$ solution; the $\mathrm{pH}$ study was carried out in the $0-7$ interval, adjusting the $\mathrm{pH}$ values required by adding a $1.0 \mathrm{~mol} \mathrm{~L}^{-1} \mathrm{HNO}_{3}$ solution along with $1.0 \mathrm{~mol} \mathrm{~L}^{-1} \mathrm{KOH}$ : the results are shown in Fig. 4 . As can be observed, the potential remained constant at low $\mathrm{pH}$ with a behavior similar to that observed with other types of ISEs [30,31]. The potential change at pH's above 5 can be due to formation of $\mathrm{Hg}$ (II) hydroxo complexes, see below. Thus, the $0-5$ interval can be taken to be the working $\mathrm{pH}$ interval of the sensor propounded, as in this interval the sensor does not respond to $\mathrm{H}^{+}$ions, which agrees closely with previous studies [27], where the empirical tests showed formation of the $\mathrm{Hg}$ (II)-PenDTF complex at acid pHs.

\subsection{Effect of renewal of the electrode surfaces by mechanical} polishing

Due to the electrode design, see Section 2.2, its surface can be renewed by lightly abrading with emery paper; therefore, it becomes important to compare the calibration plots before and 




Fig. 4. Effect of $\mathrm{pH}$ on the electrode $\mathrm{Hg}(\mathrm{II})$-ISE-PenDTF 1.

Table 2

Reproducibility of the electrode $\mathrm{Hg}(\mathrm{II})$-ISE-PenDTF 1, as a function of the number of times that its surfaces was renewed by mechanical polishing, obtained from the calibration plots in Fig. 5.

\begin{tabular}{|c|c|c|c|}
\hline $\begin{array}{l}\text { Times that the surfaces } \\
\text { was renewed }\end{array}$ & $\begin{array}{l}\text { Slope } \\
\left(\mathrm{mV} \text { decade }^{-1}\right)\end{array}$ & $\begin{array}{l}10^{7} \mathrm{LOD}^{-1} \\
\left(\mathrm{~mol} \mathrm{~L}^{-1}\right)\end{array}$ & $\begin{array}{l}\text { Linear interval } \\
\left(\mathrm{mol} \mathrm{L}^{-1}\right)\end{array}$ \\
\hline $\mathbf{0}$ & $32.6 \pm 0.5$ & $6.9 \pm 3.5$ & $5.3 \times 10^{-7}-0.01$ \\
\hline 1 & $34.5 \pm 0.7$ & $7.9 \pm 3.4$ & $5.3 \times 10^{-7}-0.01$ \\
\hline 2 & $32.2 \pm 0.4$ & $6.8 \pm 1.5$ & $5.3 \times 10^{-7}-0.01$ \\
\hline 3 & $34.6 \pm 0.4$ & $7.0 \pm 3.5$ & $5.3 \times 10^{-7}-0.01$ \\
\hline 4 & $34.1 \pm 0.5$ & $4.2 \pm 3.1$ & $5.3 \times 10^{-7}-0.01$ \\
\hline
\end{tabular}

after electrode renewal by abrasion. The potentiometric response of the sensors was tested within $1.0 \times 10^{-9}$ to $1.0 \times 10^{-2} \mathrm{~mol} \mathrm{~L}^{-1} \mathrm{Hg}$ (II) concentrations range in $1.0 \mathrm{~mol} \mathrm{~L}^{-1} \mathrm{HClO}_{4}$, after several consecutive surface renewals. Table 2 summarizes the results found regarding the analytical features displayed by electrode $\mathrm{Hg}$ (II)-ISEPenDTF 1. From these data it is possible to note that the average slope was $33.7 \pm 1.0 \mathrm{mV}$ decade $^{-1}$ while the variation coefficient obtained was $3 \%$ for the linear part of the plots, see Fig. 5. From these results one may conclude that it is very recommendable to abrade the electrode previous to perform a $\mathrm{Hg}(\mathrm{II})$ quantification.

\subsection{Selectivity}

The most important characteristic of an ISE is its selective response capacity to a primary ion in the presence of other ions. This parameter is directly related to the thermodynamic equilibrium at the surface/solution interphase. The selectivity of the electrode containing the ligand PenDTF was tested by potentiometry with different divalent cations like: $\mathrm{Ca}(\mathrm{II}), \mathrm{Ni}(\mathrm{II}), \mathrm{Cd}(\mathrm{II}), \mathrm{Mg}$ (II), $\mathrm{Cu}(\mathrm{II}), \mathrm{Co}(\mathrm{II}), \mathrm{Ba}(\mathrm{II})$ and $\mathrm{Hg}(\mathrm{II})$, the slope assessment for the majority of cations tested showed values much lower than expected from Nernstian behavior; however, for the $\mathrm{Hg}$ (II) a linear potentiometric response was observed with a value closer to such behavior, within a wide concentration range and a low detectionc limit.

The potentiometric selectivity coefficient (as measured in terms of the potentiometric selectivity constant $K_{i, j}^{P o t}$ ) that describes the preference of the electrode suggested to an interfering ion in the presence of $\mathrm{Hg}$ (II), was determined by means of the fixed interference method, which is based on the NikolskyEisenman semiempiric equation [29,32], as follows:

$E_{\mathrm{ISE}}=E^{0}+\frac{R T}{z_{i} F} \ln \left[a_{i}+\sum K_{i, j}^{P o t} a_{j}^{z_{i} / z_{j}}\right]$

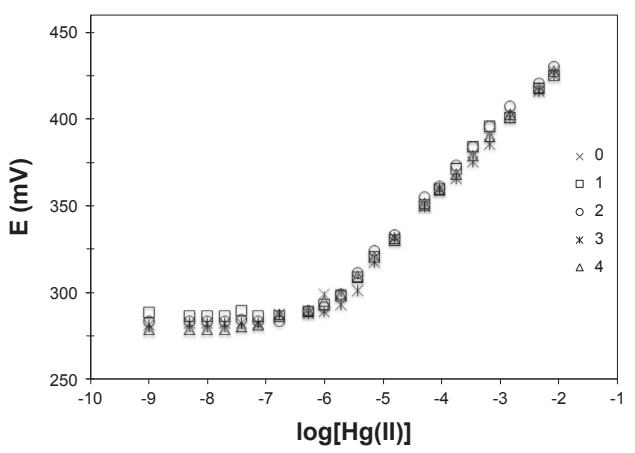

Fig. 5. Calibration curve of the electrode $\mathrm{Hg}$ (II)-ISE-PenDTF 1, as a function of the number of times, indicated in the Fig., that its surfaces was renewed by mechanical polishing.

Table 3

Potentiometric selectivity coefficient of various interfering ions $K_{H g(I I), j}^{\mathrm{Pot}}$.

\begin{tabular}{lr}
\hline Interfering ion & \multicolumn{1}{l}{$K_{\mathrm{Hg}(\mathrm{II}) j}^{\text {Pot }}$} \\
\hline $\mathrm{Ba}(\mathrm{II})$ & $2.6 \times 10^{-5} \pm 2.4 \times 10^{-6}$ \\
$\mathrm{Ca}(\mathrm{II})$ & $6.3 \times 10^{-5} \pm 5.5 \times 10^{-6}$ \\
$\mathrm{Co}(\mathrm{II})$ & $0.10 \times 10^{-5} \pm 1.5 \times 10^{-7}$ \\
$\mathrm{Mg}(\mathrm{II})$ & $20 \times 10^{-5} \pm 2.7 \times 10^{-5}$ \\
$\mathrm{Cu}(\mathrm{II})$ & $1.1 \times 10^{-5} \pm 8.4 \times 10^{-7}$ \\
$\mathrm{Cd}(\mathrm{II})$ & $4.8 \times 10^{-5} \pm 2.9 \times 10^{-6}$ \\
$\mathrm{Ni}(\mathrm{II})$ & $5.4 \times 10^{-5} \pm 5.3 \times 10^{-6}$ \\
\hline
\end{tabular}

where $E_{\mathrm{ISE}}$ is the measured potential, $E^{0}$ the standard potential, $a_{i}$ and $a_{j}$ the activities of the primary and interfering ions, respectively, $z_{i}$ the change of primary ion, $z_{j}$ the change of the interfering ion, $F$ the Faraday constant, $R$ is the universal gas constant and $T$ the absolute temperature. In this method, the primary ion concentration is varied in $\mathrm{HClO}_{4} 1.0 \mathrm{~mol} \mathrm{~L}^{-1}$ solution whereas the secondary ion concentration is kept constant at $1.0 \times 10^{-3} \mathrm{~mol} \mathrm{~L}^{-1}$, the equations for each ion can be written as

$E_{i}=E^{0}+\frac{R T}{z_{i} F} \ln \left[a_{i}\right]$

$E_{j}=E^{0}+\frac{R T}{z_{i} F} \ln \left[K_{i, j}^{P o t} a_{j}^{z_{i} / z_{j}}\right]$

Equalizing the potentials one gets

$K_{i, j}^{P o t}=\frac{a_{i}}{a_{j}^{z_{i} / z_{j}}}$

Using the fixed interference method (FIM), the selectivity coefficients were calculated and are shown in Table 3.

From the $E_{\mathrm{Hg}(\mathrm{II})-\mathrm{ISE}-\mathrm{PenDTF}}$ vs. $\log [\mathrm{Hg}$ (II)] plots and using the previous expression for $K_{i, j}^{\text {Pot }}$ the potentiometric selectivity coefficients were determined for various metal cations. As can be seen, for the majority of the cations used the selectivity coefficients values were smaller than 0.01 , which indicate that the interference produced by these ions in the sensor's operation is negligible. The electrode's selectivity sequence with the ligand PenDTF with respect to different ions, follows the order $\mathrm{Hg}$ (II) $>\mathrm{Mg}(\mathrm{II})>\mathrm{Ca}$ (II) $>\mathrm{Ni}(\mathrm{II})>\mathrm{Cd}(\mathrm{II})>\mathrm{Ba}(\mathrm{II})>\mathrm{Co}(\mathrm{II})>\mathrm{Cu}(\mathrm{II})$.

Electrodes formed by a carbon-epoxy matrix may display an anionic response due to the presence of anionic sites on its surface, which is undesirable for an ISE, thus, we examined the potentiometric 
response of the electrode $\mathrm{Hg}$ (II)-ISE-PenDTF 1 to different anions with different charges namely: $\mathrm{COO}^{-}, \mathrm{CO}_{3}{ }^{2-}, \mathrm{Cl}^{-}$and $\mathrm{SO}_{4}{ }^{2-}$. It was found that the electrode proposed here shows no change in potential at different concentrations of this sort of anions; therefore, in this case the inclusion of the PenDTF ionophore to the electrode composition may block or at least minimize this undesirable anionic response.

\subsubsection{Redox sensitivity}

In order to verify if the electrode $\mathrm{Hg}$ (II)-ISE-PenDTF 1 displays any redox sensitivity, it was immersed in an aqueous solution containing $2.0 \times 10^{-3} \mathrm{~mol} \mathrm{~L}^{-1} \mathrm{Hg}$ (II), at open circuit condition, during $48 \mathrm{~h}$. No changes were observed in the solution or at the electrode surface. Moreover, two cyclic voltammetry experiments, not shown, were also performed in the systems: $\mathrm{Hg}(\mathrm{II})$-ISE-PenDTF $1 / 2 \mathrm{mM} \mathrm{Hg}$ (II), $1 \mathrm{M} \mathrm{HClO}_{4}$ and carbon-epoxy matrix/2 $\mathrm{mM} \mathrm{Hg}$ (II), $1 \mathrm{M} \mathrm{HClO}_{4}$. From these electrochemical experiments it was found that the presence of the ionophore in the ISE avoids the electrochemical reduction and oxidation of mercury in solution. From these experiments it is possible to conclude that the electrode $\mathrm{Hg}$ (II)-ISE-PenDTF 1 does not display any redox sensitivity.

\subsection{Analytic application}

\subsubsection{Potentiometric titration}

The $\mathrm{Hg}$ (II)-ISE-PenDTF was used as an indicating electrode during the potentiometric titration of $10 \mathrm{ml}$ of an aqueous solution containing $1.0 \times 10^{-3} \mathrm{~mol} \mathrm{~L}^{-1} \mathrm{Hg}\left(\mathrm{NO}_{3}\right)_{2}$ using $1.0 \times 10^{-2} \mathrm{~mol} \mathrm{~L}^{-1}$ EDTA (Y) as titrating solution, both at $\mathrm{pH} 0$ and 4 with $\mathrm{HClO}_{4}$. The resulting plots are presented in Fig. 6, where a protracted inflection point can be observed, which strongly suggests that the generalized titration reaction $\mathrm{Hg}(\mathrm{II})^{\prime}+Y^{\prime} \Rightarrow \mathrm{Hg}(\mathrm{II}) Y^{\prime}$, where the terms $\mathrm{Hg}(\mathrm{II})^{\prime}, \mathrm{Y}^{\prime}$ and $\mathrm{Hg}(\mathrm{II}) \mathrm{Y}^{\prime}$, that represent generalized species $[33,34]$, are not quite quantitative.

Fig. 7 shows the predominance zone diagrams (PZD) of the generalized first order species $\mathrm{Y}^{\prime}$, see Fig. $7 \mathrm{a}$, and $\mathrm{Hg}(\mathrm{II})^{\prime}, \mathrm{Hg}(\mathrm{II}) \mathrm{Y}^{\prime}$, see Fig. $7 \mathrm{~b}$, for the system $\mathrm{Hg}(\mathrm{II}) / \mathrm{H}_{2} \mathrm{O} / \mathrm{H}^{+}$and the existence predominance diagram (EPD) of the species of $\mathrm{Hg}(\mathrm{II})$, see Fig. 7c; these diagrams were constructed using the methodology described by Rojas-Hernández et al. [33,34], which allowed observing the species participating in the titration reaction, at $\mathrm{pH}=0$; therefore, it can be concluded that for $\mathrm{pH}=0$ the representative titration reaction is

$\mathrm{Hg}^{2+}+\mathrm{H}_{4} \mathrm{Y} \Rightarrow \mathrm{HgYH}^{-}+3 \mathrm{H}^{+}$

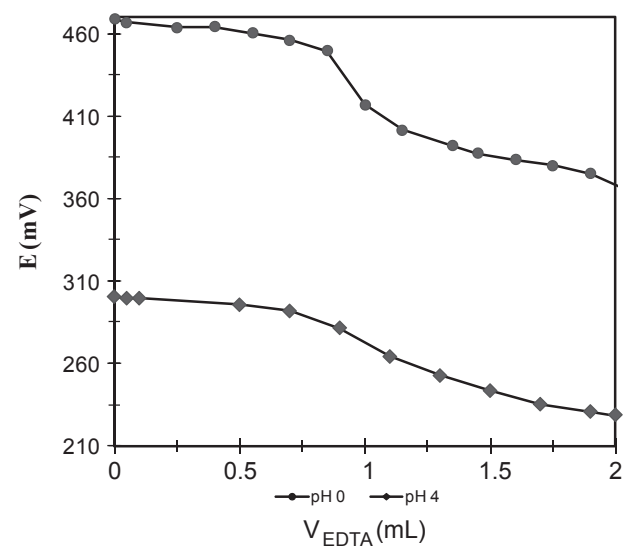

Fig. 6. Potentiometric titration plots of $10 \mathrm{~mL}$ of $\mathrm{Hg}^{2+} 1.0 \times 10^{-3} \mathrm{~mol} \mathrm{~L}^{-1}$ solution with $1.0 \times 10^{-2} \mathrm{~mol} \mathrm{~L}^{-1}$ of EDTA. a

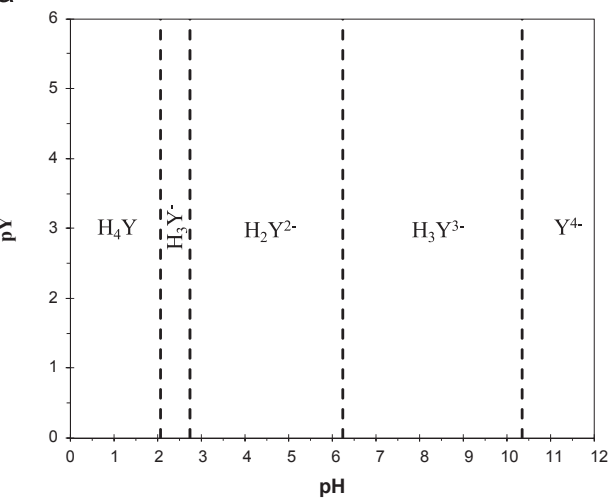

b

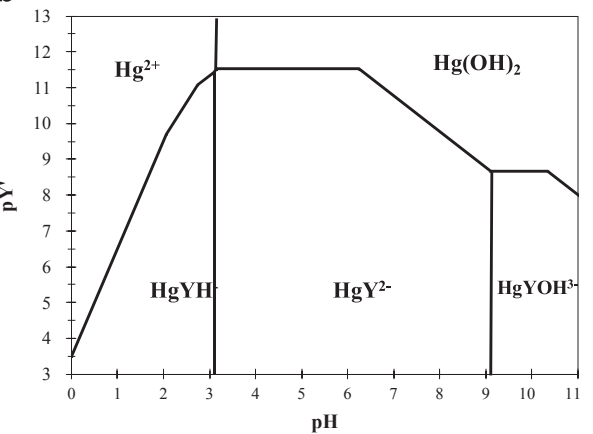

C

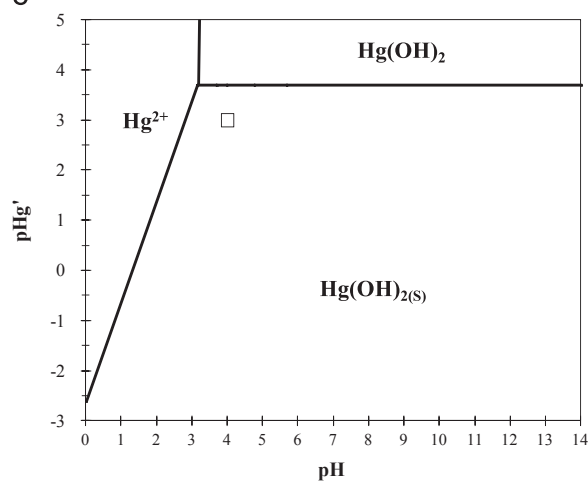

Fig. 7. Predominance zone diagrams (PZD) for the system $\mathrm{Hg}(\mathrm{II}) / \mathrm{H}_{2} \mathrm{O}$. (a) $\mathrm{PZD}$ for $\mathrm{Y}^{\prime}$ (b) PZD for $\mathrm{Hg}(\mathrm{II})^{\prime}$ and (c) existence predominance diagram (EPD), for $\mathrm{Hg}(\mathrm{II})$; the symbol $\square$ represents the point $\left(\mathrm{pH}, \mathrm{pHg}^{\prime}\right)=(4,3)$.

with $\log K_{r}^{\prime}=3.5$. Thus, in agreement with Ringbom [35], a reaction of this type is quantitative if $K_{r}^{\prime} C_{o} \geq 10^{5}$ where $C_{0}$ is the initial $\mathrm{Hg}$ (II) concentration. As in this case the $K_{r}^{\prime} C_{o}=10^{0.5}$, the reaction is not quantitative.

At $\mathrm{pH} \mathrm{4}$, the representative titration reaction is

$\mathrm{Hg}(\mathrm{OH})_{2}+\mathrm{H}_{2} \mathrm{Y}^{2-} \Rightarrow \mathrm{HgY}^{2-}+2 \mathrm{H}_{2} \mathrm{O}$ 
a

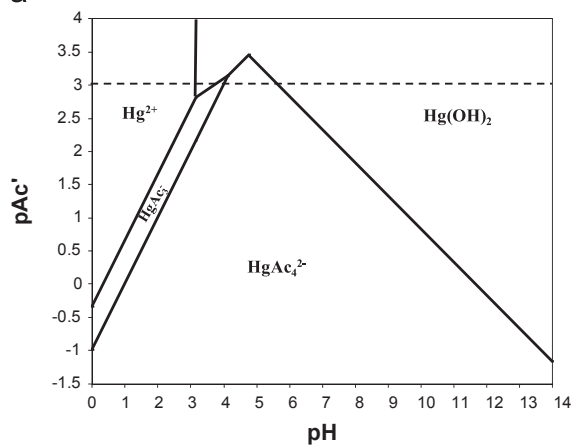

b

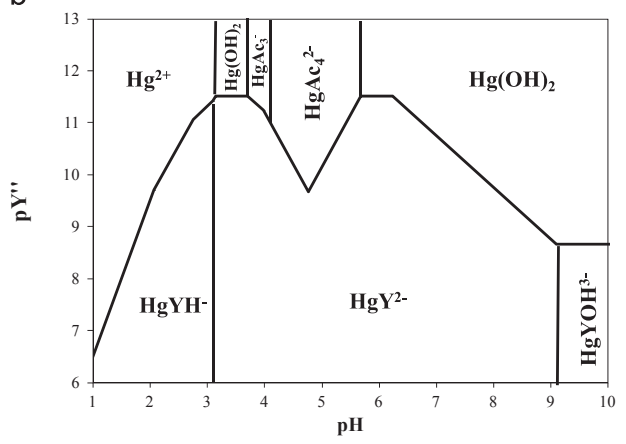

C

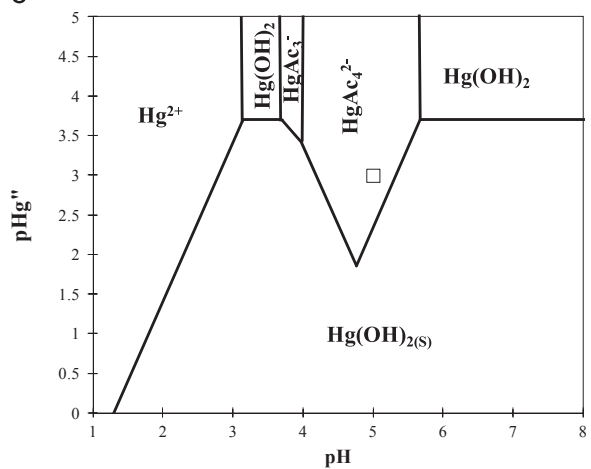

Fig. 8. Predominance zone diagrams (PZD) for the system $\mathrm{Hg}(\mathrm{II}) / \mathrm{Ac} / \mathrm{H}_{2} \mathrm{O}$ : (a) PZD for $\mathrm{Hg}^{\prime \prime}$, the dotted line represents the cut $\mathrm{pAc}^{\prime}=3$, (b) $\mathrm{PZD}$ for $\mathrm{HgY}^{\prime \prime}$ at $\mathrm{pAc}^{\prime}=3.0$ and (c) existence predominance diagram (EPD) for $\mathrm{Hg}(\mathrm{II})$ at $\mathrm{pAc}=3$, the symbol represents the point $\left(\mathrm{pH}, \mathrm{pHg}^{\prime \prime}\right)=(5,3)$

which has a $\log K_{\mathrm{r}}^{\prime}=11.52$, when dealing with species in solution, however Fig. 7c shows the presence of the insoluble mercury hydroxide species, which disfavors the titration reaction and abates the reaction quantitativity.

Since the formation of $\mathrm{Hg}$ (II) insoluble species decreases the quantitativity of the reaction with EDTA titration, it becomes necessary to explore the possibility of increasing the mercury solubility, then the system $\mathrm{Hg}$ (II)/Ac/ $\mathrm{H}_{2} \mathrm{O} / \mathrm{H}^{+}$was analyzed in order to establish the optimal conditions that guarantee the quantitativity of the mercury titration reaction with EDTA. Fig. 8 presents the PZDs of the generalized species at second order $\mathrm{Hg}(\mathrm{II})^{\prime \prime}$, see Fig. 8a, and $\mathrm{Hg}(\mathrm{II}) \mathrm{Y}^{\prime \prime}$, see Fig. 8b, for the system $\mathrm{Hg}(\mathrm{II}) / \mathrm{Ac} / \mathrm{H}_{2} \mathrm{O} / \mathrm{H}^{+}$; the horizontal dashed line in Fig. 8a indicates the first order buffering effect at $\mathrm{pAc}^{\prime}=3.0$.

Fig. 8c presents the EPD diagram of the $\mathrm{Hg}(\mathrm{II})^{\prime \prime}$ species; this diagram was also built using the methodology described by RojasHernández et al. [33,34]. From this diagram it can be preliminary concluded that the mercury hydroxide does not precipitate at $\mathrm{pHg}(\mathrm{II})^{\prime \prime}=3$ after imposing a $\mathrm{pH}$ at 5.0 in the system with $1.0 \times 10^{-3} \mathrm{~mol} \mathrm{~L}^{-1} \mathrm{HAc} / \mathrm{Ac}^{-}$buffer.

From Figs. 7a and $8 \mathrm{a}$ and $\mathrm{b}$ it can be also concluded that the $\mathrm{Y}$ $"=\mathrm{H}_{2} \mathrm{Y}^{2-}, \mathrm{Hg}(\mathrm{II})^{\prime \prime}=\mathrm{HgAc}_{4}{ }^{2-}$ and $\mathrm{Hg}(\mathrm{II}) \mathrm{Y}^{\prime \prime}=\mathrm{HgY}^{2-}$. This way, the $\mathrm{Hg}$ (II) representative titration reaction using EDTA at pH 5.0 imposed with $1.0 \times 10^{-3} \mathrm{~mol} \mathrm{~L}^{-1} \mathrm{HAc} / \mathrm{Ac}^{-}$buffer is

$\mathrm{HgAc}_{4}^{2-}+\mathrm{H}_{2} \mathrm{Y}^{2-} \Rightarrow \mathrm{HgY}^{2-}+4 \mathrm{Ac}^{-}+2 \mathrm{H}^{+}$

where the reaction constant is given by

$K_{r}=\frac{\left[\mathrm{HgY}^{2-}\right]\left[\mathrm{AC}^{-}\right]^{4}\left[\mathrm{H}^{+}\right]^{2}}{\left[\mathrm{HgAc}_{4}^{2-}\right]\left[\mathrm{H}_{2} \mathrm{Y}^{2-}\right]}$

If the concentration of acetate ions and protons is imposed, then $K_{r}$ can be expressed as the biconditional constant $K_{r}$ " where $\log K_{r}^{\prime \prime}=-11.84+4 \mathrm{pAc}^{\prime}+2 \mathrm{pH}=10.16$ and because in this case $K_{r}^{\prime \prime}$ $C_{o}=10^{7.16}$, the reaction is quantitative.

The results of the $\mathrm{Hg}$ (II) titration are shown in Fig. 9. The titrant added caused a potential diminution as a result the $\mathrm{Hg}$ (II) ion concentration decreases due to formation of an EDTA complex. The $\mathrm{Hg}$ (II) ions quantity in solution can be determined precisely from the resulting titration plots, where from the volume obtained at the equivalence point was $0.97 \pm 6.0 \times 10^{-3} \mathrm{~mL}$, this gives then $\mathrm{Hg}(\mathrm{II})$ concentration of $(9.7 \pm 0.7) \times 10^{-5} \mathrm{~mol} \mathrm{~L}^{-1}$.

Additionally, Fig. 9 shows the titration curve of the same concentration of $\mathrm{Hg}$ (II) and in the same conditions using a commercial mercury cup electrode: overlapping the curves shows the similarity of the equivalence points, indicating that the sensor proposed here can be used adequately for titration of $\mathrm{Hg}$ (II) ions.

\subsection{Comparison with the literature}

Table 4 shows some $\mathrm{Hg}(\mathrm{II})$ selective electrodes reported in the literature, where the type of compound used as recognition element can be found, the electrode configuration and some analytic parameters such as: their Nernstian response as well as the working interval, so that a good comparison can be established with the one

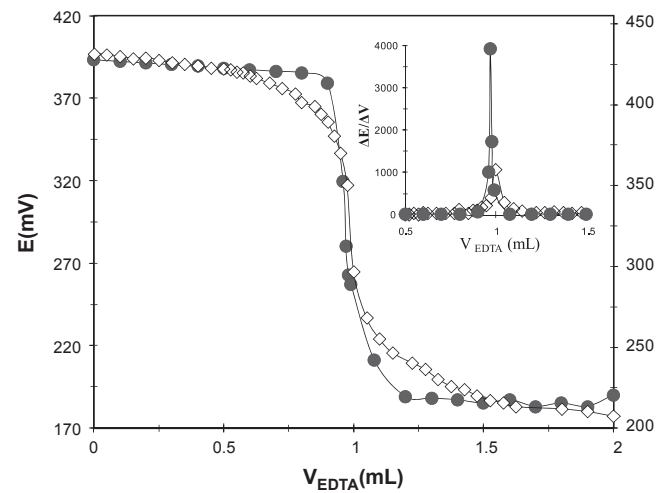

Fig. 9. Potentiometric titration curve of $\mathrm{Hg}^{2+} 1.0 \times 10^{-3} \mathrm{~mol} \mathrm{~L}^{-1} 10 \mathrm{ml}$ solution with $1.0 \times 10^{-2} \mathrm{~mol} \mathrm{~L}^{-1}$ of EDTA. (•) Using the sensor Hg(II)-ISE-PenDTF 1 and (口) using a mercury cup electrode (secondary axis) 
Table 4

Recent $\mathrm{Hg}(\mathrm{II})$-selective electrodes proposed in the literature.

\begin{tabular}{|c|c|c|c|c|c|c|}
\hline Ref. & Ionophore & $\begin{array}{l}\text { Type of } \\
\text { electrode }\end{array}$ & $\begin{array}{l}\text { Slope } \\
\left(\mathrm{mV} \mathrm{decade}^{-1}\right)\end{array}$ & $\begin{array}{l}\text { Linear interval } \\
\left(\mathrm{mol} \mathrm{L}^{-1}\right)\end{array}$ & $\mathrm{pH}$ range & Selectivity $\left(K_{\mathrm{Hg}(\mathrm{II}, j}^{\mathrm{Pot}}\right)$ \\
\hline [36] & $\begin{array}{l}\text { Dithiophosphorus macrocyclic } \\
\text { compound }\end{array}$ & Membrane & 30.2 & $1.0 \times 10^{-6}-1.0 \times 10^{-1}$ & $3.0-6.0$ & $\begin{array}{l}\mathrm{Pb}^{2+} ; 6.4 \times 10^{-2}, \mathrm{Cd}^{2+} ; 6.2 \times 10^{-3}, \mathrm{Ni}^{2+} ; 4.2 \times 10^{-3}, \mathrm{Co}^{2+} ; \\
6.9 \times 10^{-3}, \mathrm{Mg}^{2+} ; 7.3 \times 10^{-4}, \mathrm{Fe}^{2+} ; 8.2 \times 10^{-4}\end{array}$ \\
\hline [37] & $\begin{array}{l}\text { Dibenzyl calix[4]arene- } \\
\text { dithiacrown }\end{array}$ & Membrane & 29.5 & $3.0 \times 10^{-7}-3.0 \times 10^{-2}$ & - & $\begin{array}{l}\mathrm{Cd}^{2+} ; 5.5 \times 10^{-3}, \mathrm{Co}^{2+} ; 5.2 \times 10^{-4}, \mathrm{Cu}^{2+} ; 4.1 \times 10^{-4}, \mathrm{Ni}^{2+} ; \\
2.8 \times 10^{-4}, \mathrm{~Pb}^{2+} ; 7.4 \times 10^{-3}, \mathrm{Zn}^{2+} ; 7.4 \times 10^{-4}\end{array}$ \\
\hline [38] & $\begin{array}{l}\text { 8,17-bis(pyren-1-ylmethyl)- } \\
6,7,8,9,15,16,17,18- \\
\text { octahydrodibenzo[f,m][1,8,4,11] } \\
\text { dithiodiazacyclotetradecine }\end{array}$ & Membrane & 27.6 & $1.0 \times 10^{-6}-1.0 \times 10^{-2}$ & $4.0-4.5$ & - \\
\hline [39] & $\begin{array}{l}\text { Benzothiazole azocalix[4]arene } \\
\text { (BTC4) }\end{array}$ & $\begin{array}{l}\text { Carbon } \\
\text { paste }\end{array}$ & - & $1.0 \times 10^{-4}-1.0 \times 10^{-2}$ & - & - \\
\hline [40] & $\begin{array}{l}26,27,28 \text {-tris-benzyloxy-25- } \\
\text { hydroxy-5,11,17,23-tetra-tert- } \\
\text { butyl-calix[4]arene }\end{array}$ & Membrane & 29.4 & $1.0 \times 10^{-6}-1.0 \times 10^{-1}$ & $1.5-4.0$ & $\begin{array}{l}\mathrm{Ca}^{2+} ; 5.3 \times 10^{-3}, \mathrm{Sr}^{2+} ; 3.8 \times 10^{-4}, \mathrm{Ba}^{2+} ; 1.9 \times 10^{-3}, \mathrm{Co}^{2+} ; \\
3.5 \times 10^{-3}, \mathrm{Zn}^{2+} ; 1.3 \times 10^{-3}, \mathrm{~Pb}^{2+} ; 5.1 \times 10^{-3}, \mathrm{Cd}^{2+} ; \\
4.7 \times 10^{-3}, \mathrm{Ni}^{2+} ; 7.4 \times 10^{-2}, \mathrm{Cu}^{2+} ; 5.9 \times 10^{-3}, \mathrm{Sn}^{2+} ; \\
1.2 \times 10^{-3}\end{array}$ \\
\hline [41] & $\begin{array}{l}\mathrm{N}, \mathrm{N}^{\prime} \text {-bis(pyridin-2-ylmethyl)-p- } \\
\text { tert-butylcalix[4]arene-diaza- } \\
\text { crown }\end{array}$ & Membrane & $-27.8,-52.7$ & $1.0 \times 10^{-6}-1.0 \times 10^{-2}$ & - & - \\
\hline [42] & $\begin{array}{l}\mathrm{N}, \mathrm{N}^{\prime} \text {-bis(salicylaldehyde)- } \\
\text { phenylendiamine }\end{array}$ & Membrane & 58.8 & $3.2 \times 10^{-7}-3.2 \times 10^{-4}$ & $3.8-7.8$ & $\begin{array}{l}\mathrm{Cd}^{2+} ; 2.3 \times 10^{-3}, \mathrm{Mg}^{2+} ; 1.9 \times 10^{-4}, \mathrm{Ca}^{2+} ; 6.1 \times 10^{-4}, \mathrm{Ba}^{2} \\
{ }^{+} ; 3.9 \times 10^{-4}, \mathrm{Zn}^{2+} ; 2.2 \times 10^{-3}, \mathrm{Ni}^{2+} ; 5.3 \times 10^{-4}, \mathrm{Li}^{2+} ; \\
2.3 \times 10^{-4}, \mathrm{Co}^{2+} ; 4.1 \times 10^{-3}, \mathrm{Cu}^{2+} ; 1.4 \times 10^{-1}, \mathrm{~Pb}^{2+} ; \\
1.1 \times 10^{-2}\end{array}$ \\
\hline [43] & $\begin{array}{l}\text { 1-furan-2-yl-4-(4-nitrophenyl)-2- } \\
\text { phenyl-5H-imidazole-3-oxide }\end{array}$ & Membrane & 29.3 & $1.0 \times 10^{-5}-1.0 \times 10^{-1}$ & $1.0-4.0$ & - \\
\hline [44] & $\begin{array}{l}\text { 1,3,bis(2-methoxybenzene) } \\
\text { triazene (MBT) }\end{array}$ & Membrane & 30.2 & $1.0 \times 10^{-7}-1.0 \times 10^{-2}$ & $2.6-4.2$ & $\begin{array}{l}\mathrm{Zn}^{2+} ; 1.8 \times 10^{-3}, \mathrm{~Pb}^{2+} ; 3.8 \times 10^{-3}, \mathrm{Ni}^{2+} ; 2.3 \times 10^{-3}, \mathrm{Cd}^{2+} \\
5.8 \times 10^{-3}, \mathrm{Mg}^{2+} ; 2.2 \times 10^{-5}\end{array}$ \\
\hline [45] & Oxadiazaphosphepina derivatives & Membrane & 29.4 & $1.0 \times 10^{-6}-1.0 \times 10^{-1}$ & $0.9-4.5$ & - \\
\hline This work & $\begin{array}{l}\text { O,O'-(2,2'-bifenylen) } \\
\text { dithiophosphate pentyl }\end{array}$ & Composite & 33.7 & $5.3 \times 10^{-7}-1.0 \times 10^{-2}$ & $0.0-5.0$ & Table 3 \\
\hline
\end{tabular}

reported herein. The sensor proposed in this work presents useful advantages, not just in construction but also in actual working, as well as in synthesis of the recognition element. Because this is an inner solid contact electrode, hence an internal reference electrode is eliminated, just as the reference solution, thus facilitating construction, maintenance and ease of handling.

The sensor exhibits another relevant advantage with respect to those based on polymeric membranes found in the literature, regarding such components as the plasticizer and the ion exchanger that are essential to prepare the polymeric blend. Other comparative advantage of this electrode as referred to those based on polymeric membranes, is that if the latter were to suffer some damage or were contaminated, they would not be regenerated easily, whereas that reported here enables proper regeneration of the sensor's active sites on the surface, that may have gotten inactive or blocked, merely by lightly grinding away the surface in order to obtain a new set of fully available active sites. The ionophore used in this work is approximately 60 times less expensive to that of obtained as compared to the polymeric membranes of some of the sensors referred here [36,38-40], which have the added disadvantage of not showing better analytic parameters.

Lastly, comparing with other devices already reported that are not based on potentiometric measurements, the electrode herein proposed is even more advantageous because the synthesis routes of chromosensor compounds elevate the overall costs of sensors. Ref. [39] reports a chromosensor that is approximately 590 times more expensive to obtain, with respect to the recognition element used in the sensor reported here.

\section{Conclusions}

The electrode based on $0, \mathrm{O}^{\prime}$-(2,2'-biphenylene)dithiophosphate pentyl (PenDTF) showed a good Nernstian response for $\mathrm{Hg}$ (II) (even in the presence of divalent metal ions) within an ample concentration range. The applicable $\mathrm{pH}$ interval, lower detection limit, and potentiometric selectivity coefficients of the sensor, strongly suggest that the sensor propounded is comparable with those of other methods used for the determination of this ion, giving additional advantages with respect to other sensors proposed in the literature [35,37-39]. The sensor based on PenDTF exhibits different advantages like in the synthesis cost of the ionophore, in the design of the renewable inner solid contact that can be used for various experiments after light abrading and the elimination of the inner reference solution that turns the sensor maintenance-free, thus favoring safe storage. The sensor examined can be used as an indicator electrode in determining $\mathrm{Hg}$ (II) ions, since its response is comparable with commercial electrodes using the same principle.

\section{Acknowledgments}

The authors are grateful to CONACyT for the student grant 240838 of Juárez-Gómez and 175616 of Pérez-García. MTRS, MEPH and CAGV wish to thank the SNI for the distinction of their membership and the stipend received. Thanks also to CONACyT for support through project 82932. Authors are indebted to Dr. Manuel Palomar-Pardavé and Dr. Mario Romero-Romo for fruitful discussion regarding this paper.

\section{References}

[1] S. Engst, S.M. Miller, Biochemistry 38 (1999) 3519-3529.

2] H. Qian, L. Sahlman, P.O. Eriksson, C. Hambraeus, U. Edlund, I. Sethson, Biochemistry 37 (1998) 9316-9322.

3] R.K. Mahajan, R. Kaur, V. Bhalla, M. Kumar, T. Hattori, S. Miyano, Sens. Actuators B 130 (2008) 290-294. 
[4] I. Bontidean, A. Mortari, S. Leth, N.L. Brown, U. Karlson, M.M. Larsen, J. Vangronsveld, P. Corbisier, E. Csoregi, Environ. Pollut. 131 (2004) 255-262. J.L. Hobman, N.L. Brown, in: A. Sigel, H. Sigel (Eds.), Metal Ions in Biological Systems, Marcel Dekker, New York, 1997.

[6] J.L. Hobman, J.R. Wilson, N.L. Brown, D.R. Lovley, Environmental Microbe Metal Interactions, ASM Press, Herndon, 2000

7] R.D. Maeco, G. Clarke, B. Pejctc, Electroanalysis 19 (1987) 1987-2001

8] X. Yu, Z. Zhou, Y. Wang, Y. Liu, Q. Xie, D. Xiao, Sens. Actuators B 123 (2007) $352-358$.

[9] M.E. Páez-Hernández, K. Aguilar-Arteaga, M. Valiente, M.T. Ramírez-Silva, M. Romero-Romo, M. Palomar-Pardavé, Anal. Bioanal. Chem. 380 (2004) 690-697.

[10] M.E. Páez-Hernández, K. Aguilar-Arteaga, C. Galán-Vidal, M.T. Ramírez-Silva, M. Romero-Romo, M. Palomar-Pardavé, Environ. Sci. Technol. 39 (2005) 7667-7670.

[11] Z.L. Peng, F. Qu, Q. Song, J.M. Lin, Electrophoresis 26 (2005) 3333-3340.

[12] H. Hintelmann, R.D. Evans, J.V. Villeneuve, J. Anal. At. Spectrom. 10 (1995) 619-624.

[13] I. Palchetti, S. Majid, A. Kicela, G. Marrazza, M. Mascini, Int. J. Environ. Anal. Chem. 83 (2003) 701-711.

[14] P. Buhlmann, E. Pretsch, E. Bakker, Chem. Rev. 98 (1998) 1593-1687.

[15] F. Bakhtiarzadeh, S.A. Ghani, J. Electroanal. Chem. 624 (2008) 139-143.

[16] V.K. Gupta, S. Chandra, H. Lang, Talanta 66 (2005) 575-580.

17] A.K. Singh, R.P. Singh, S. Mehtab, J. Inclusion Phenom. Macrocycl. Chem. 60 (2008) 9-15.

[19] N.A. Kiol, F . Akhtar Inamuddin, Anal. Sci. 24 (2008) 881-887. 122 (2007) 187-194.

[20] M.T. Lai, J.S. Shih, Analyst 111 (1986) 891-895.

[21] V.K. Gupta, A.K. Singh, A. Al Khayat, B. Gupta, Anal. Chim. Acta 590 (2007) $81-90$

[22] R.K. Mahajan, I. Kaur, T.S. Lobana, Talanta 59 (2003) 101-105.

[23] L. Perez-Marin, E. Otazo-Sanchez, G. Macedo-Miranda, P. Avila-Perez, J. A. Chamaro, H.L. Lopez-Valdivia, Analyst 125 (2000) 1787-1790.

[24] R.K. Mahajan, R. Kaur, I. Kaur, V. Sharma, M. Kumar, Anal. Sci. 20 (2004) $811-814$
[25] M. Castro, J. Cruz, E. Otazo, L.P. Marın, J. Phys. Chem. A 107 (2003) 9000-9007. L.P. Marın, M. Castro, E. Otazo, G.A. Cisneros, Int. J. Quantum Chem. 80 (2000) 609-622.

[27] F. Pérez-García, C.A. Galán-Vidal, J.G. Albarado-Rodríguez, M.E. PáezHernández, N. Andrade-López, M.T. Ramírez-Silva, Sep. Sci. Technol. 48 (2013) 736-740.

[28] F. Pérez-García, J.G. Albarado-Rodríguez, C.A. Galán-Vidal, M.E. PáezHernández, N. Andrade-López, Struct. Chem. 21 (2010) 191-196.

[29] IUPAC, Pure Appl. Chem. 48 (1976) 127-142.

30] M.H. Mashhadizadeh, M. Talakesh, M. Peste, A. Momeni, H. Hamidian, M. Mazlumd, Electroanalysis 18 (2006) 2174-2179.

[31] R.K. Mahajan, R.K. Puri, A. Marwaha, I. Kaur, M.P. Mahajan, J. Hazard. Mater 167 (2009) 237-243.

[32] Y. Umezawa, K. Umezawa, H. Sato, Pure Appl. Chem. 67 (1995) 507-518. A. Rojas Hernández, M.T. Ramírez Silva, J.G. Ibañez, I. González, J. Electrochem. Soc. 138 (2) (1991) 365-371.

[34] A. Rojas-Hernández, M.T. Ramírez-Silva, I. González, J.G. Ibañez., Anal. Chim. Acta 246 (1991) 435-441.

[35] A. Ringbom, Formación de Complejos en Química Analítica, Alhambra, Madrid, 1979.

[36] B. Patel, A. Kumar, S.K. Menon, J. Inclusion Phenom Macrocyclic Chem. 64 (2009) 101-108.

[37] Y. Yang, X. Cao, M. Surowiec, R.A. Bartsch, Tetrahedron 66 (2010) 447-454 B. Palkiran, A.D. Ozel, S. Palayan, E. Canel, U. Ocak, E. Kilic, Monatsh. Chem. 14 (2010) 829-839.

[39] H. Bingol, E. Kocabas, E. Zor, A. Coskum, Talanta 82 (2010) 1538-1542. $407-422$.

[41] Y. Kim, J. Kim, K.N. Kim, S. Chang, T.D. Chung, Anal. Sci. 25 (2009) 567-570.

[42] H.M. Abu.Shawish, J. Hazard. Mater. 167 (2009) 602-608.

[43] R.K. Mahan, R.K. Puri, A. Marwaha, I. Kaur, M.P. Mahajan, J. Hazard. Mater 167 (2009) 237-243.

[44] M.K. Rofouei, M. Mohammadi, M.B. Gholivand, Mater. Sci. Eng. 29 (2009) 2154-2159.

[45] R.K. Puri, V. Kumar, M.P. Mahajan, R.K. Mahajan, J. Inclusion Phenom Macrocyclic Chem. 69 (2011) 263-271. 